PUBLICATIONS

NISTIR 5037

\title{
Control of Gas-Metal-Arc Welding Using Arc-Light Sensing
}

R. Bruce Madigan

Timothy P. Quinn

Thomas A. Siewert

QC

100

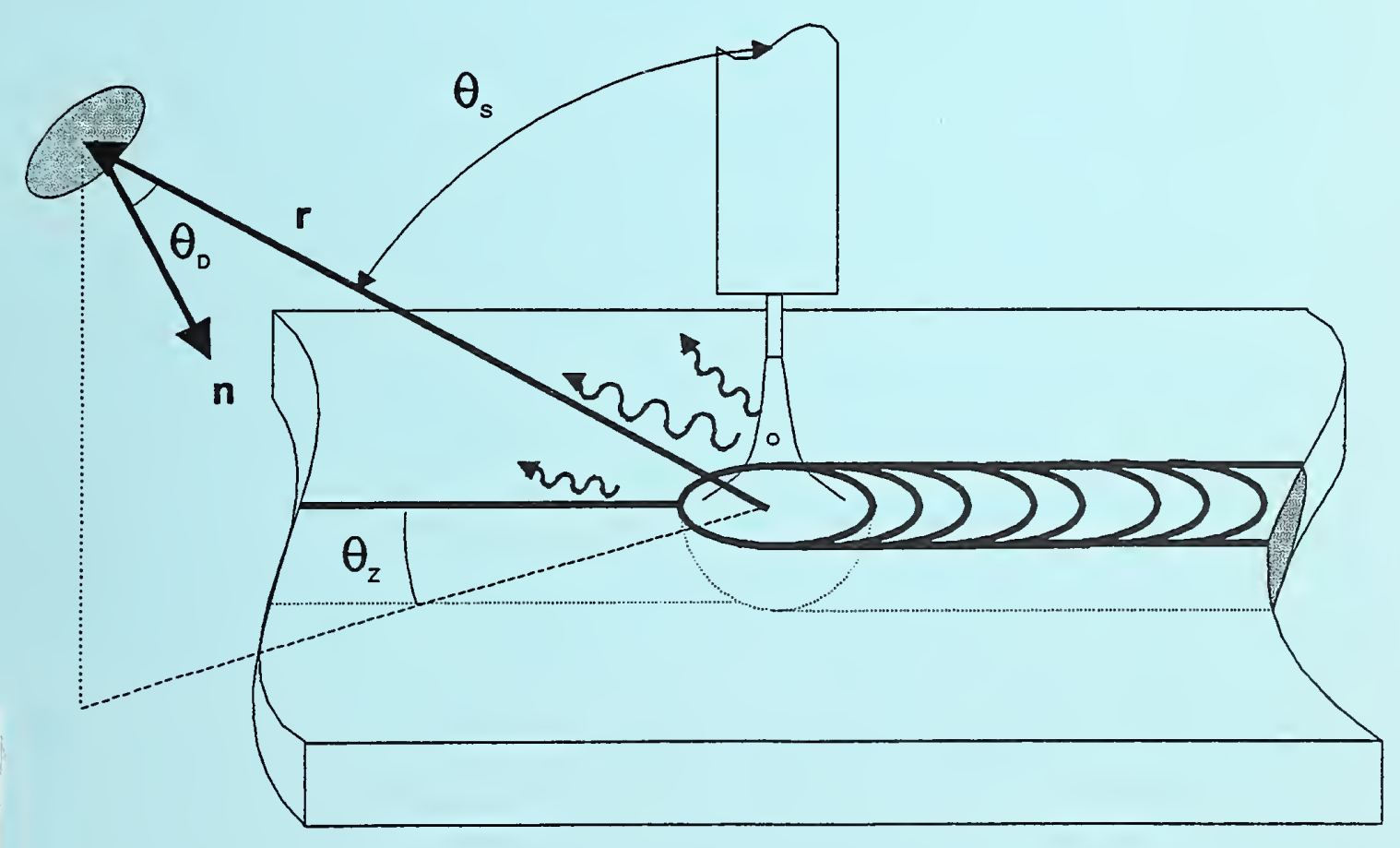

.056

N0.5037

1995 



\section{Control of Gas-Metal-Arc Welding Using Arc-Light Sensing}

R. Bruce Madigan

Timothy P. Quinn

Thomas A. Siewert

Materials Reliability Division

Materials Science and Engineering Laboratory

National Institute of Standards and Technology

Boulder, Colorado 80303-3328

November 1995

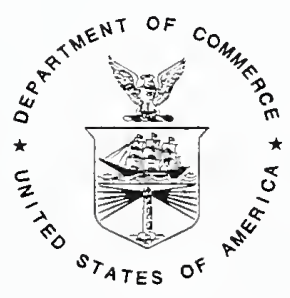

U.S. DEPARTMENT OF COMMERCE, Ronald H. Brown, Secretary TECHNOLOGY ADMINISTRATION, Mary L. Good, Under Secretary for Technology NATIONAL INSTITUTE OF STANDARDS AND TECHNOLOGY, Arati Prabhakar, Director 


\section{Contents}

List of Figures $\ldots \ldots \ldots \ldots \ldots \ldots \ldots \ldots \ldots \ldots \ldots \ldots \ldots$

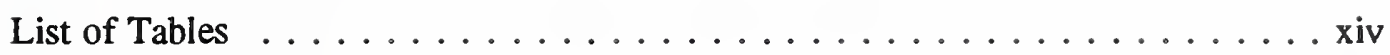

List of Symbols and Acronyms $\ldots \ldots \ldots \ldots \ldots \ldots \ldots \ldots$

1. Introduction $\ldots \ldots \ldots \ldots \ldots \ldots \ldots \ldots \ldots \ldots \ldots \ldots$

1.1 Fundamentals of Gas-Metal-Arc Welding $\ldots \ldots \ldots \ldots \ldots \ldots$

1.2 Objective and Approach $\ldots \ldots \ldots \ldots \ldots \ldots \ldots \ldots \ldots \ldots \ldots \ldots$

2. Characteristics of the Process and Process Control .......... 3

2.1 Process Variables . . . . . . . . . . . . . . . 3

2.2 Transfer Mode ..................... 5

2.3 Mathematical Modeling . . . . . . . . . . . . 5



2.3.2 Electrode Melting Rate $\ldots \ldots \ldots \ldots \ldots \ldots \ldots \ldots$

2.3.3 Process Voltage . . . . . . . . . . . . . 6

2.4 Welding-Process Control . . . . . . . . . . . . . . . 11

2.4.1 Control Block Diagrams and the Transfer Function . . . . . 12

2.4.2 Open-Loop Control . . . . . . . . . . . . . . . 14

2.4.3 Closed-Loop Control . . . . . . . . . . . . . . 14

2.4.4 Welding-Process Controller . . . . . . . . . . 16

2.5 Summary . . . . . . . . . . . . . . . . 16

3. Emission and Sensing of Arc Light . . . . . . . . . . . 17

3.1 Electrical Conduction in the Welding Arc . . . . . . . . 17

3.2 Arc-Energy Balance . . . . . . . . . . . . . . . 18

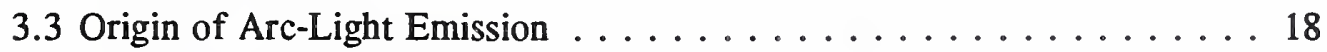

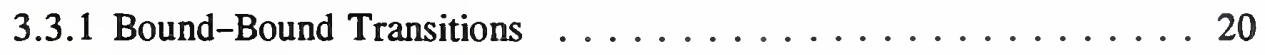

3.3.2 Bound-Free Transitions . . . . . . . . . . . . 22

3.3.3 Free-Free Transitions . . . . . . . . . . . . . 22

3.4 Experimental Observations of Arc Radiation . . . . . . . 22

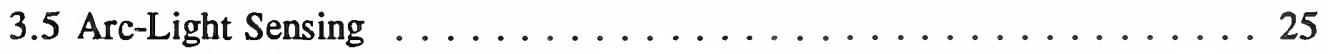

3.6 Summary . . . . . . . . . . . . . . . . . 27

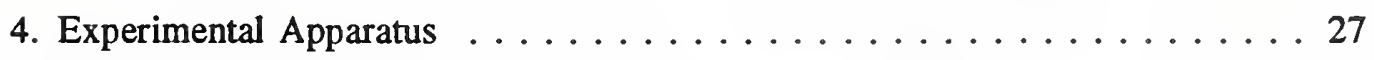

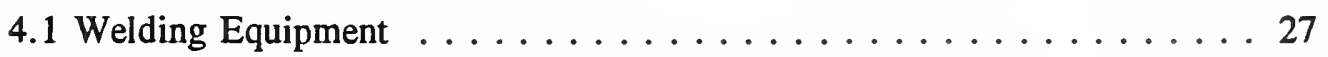

4.2 Instrumentation . . . . . . . . . . . . . 28 
5. Estimation of Arc Length $\ldots \ldots \ldots \ldots \ldots \ldots \ldots \ldots \ldots$

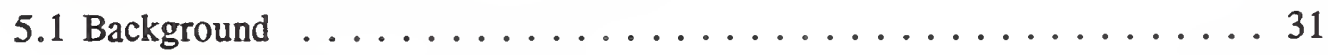

5.1 .1 Through-the-Arc Sensing . . . . . . . . . . . 31

5.1 .2 External Sensors . . . . . . . . . . . . . 33

5.1.3 Review of Preliminary Work ............. 34

5.2 Arc-Length Sensor . . . . . . . . . . . . . . . 35

5.3 Detector . . . . . . . . . . . . . . . 36

5.3.1 Location and Orientation . . . . . . . . . . 36

5.3.2 Spectral Locations for Sensing . . . . . . . . . . 38

5.4 Experimental Method . . . . . . . . . . . . 42

5.4 .1 Welding Procedure . . . . . . . . . . . . . 42

5.4 .2 Detector Configuration . . . . . . . . . . . 42

5.4.3 Data Collection and Analysis .............. 43

5.5 Results and Discussion . . . . . . . . . . . . 44

5.5.1 Correlation of Results to the Arc-Length-Sensor Model . . . . . . 44

5.5.2 Source/Detector Angle . . . . . . . . . . . . . . 49

5.5.3 Detector Orientation . . . . . . . . . . . . . 53

5.5.4 Detector Distance ................... . . 53

5.5.5 Optical Filters and Shielding Gas . . . . . . . . . 56

5.6 Advantages and Disadvantages $\ldots \ldots \ldots \ldots \ldots \ldots 64$

5.7 Summary . . . . . . . . . . . . . . . . . . . . 64

6. Control of Arc Length . . . . . . . . . . . . . 65

6.1 Background .......................... 65

6.2 Experimental Method . . . . . . . . . . . . . 66

6.3 Arc-Length Sensing. . . . . . . . . . . . . . . . 66

6.4 Characterization of the Welding Process . . . . . . . . . 66

6.5 Design of the Controller . . . . . . . . . . . . . . 68

6.6 Results and Discussion . . . . . . . . . . . . . . 69

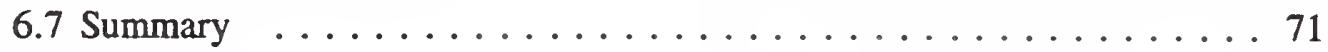

7. Detection of Droplet-Transfer Frequency $\ldots \ldots \ldots \ldots \ldots \ldots \ldots$

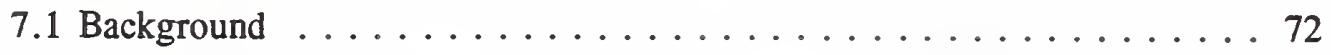

7.2 Experimental Method . . . . . . . . . . . . . 74

7.2 .1 Welding Conditions $\ldots \ldots \ldots \ldots \ldots \ldots \ldots \ldots \ldots$

7.2.2 Detector Configuration . . . . . . . . . . . . 74

7.2 .3 Data Collection . . . . . . . . . . . . . . . 74

7.2.4 Analysis of Droplet-Transfer Frequency . . . . . . . . 76

7.2.5 Analysis of Optical Filters . . . . . . . . . . 76 
7.3 Results and Discussion .................. 77

7.3.1 Measurement of Droplet Detachment ............ 77

7.3.2 Correlation of Droplet Detachment with Arc-Light-Detector Voltage . . . . . . . . . . . . . 77

7.3.3 Detection of Droplet-Transfer Frequency: Experiments . . . . . . 79

7.3 .4 Effect of Optical Filtering . . . . . . . . . . . 86

7.4 Summary . . . . . . . . . . . . . . 86

8. Control of Droplet-Transfer Frequency ... . . . . . . . . . 88

8.1 Background $\ldots \ldots \ldots \ldots \ldots \ldots \ldots \ldots$

8.2 Experimental Method ....................... 88

8.3 Droplet-Transfer-Frequency Sensor . . . . . . . . . . . . . . . 89

8.4 Characterization of the Process . . . . . . . . . . . . . . 89

8.5 Controller Design . . . . . . . . . . . . . . . . . . 92

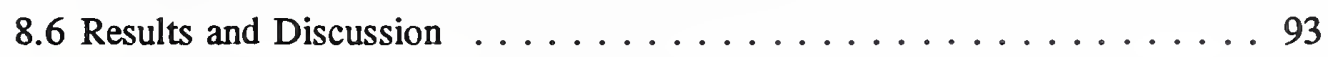

8.7 Summary . . . . . . . . . . . . . . . . . . . . . 94

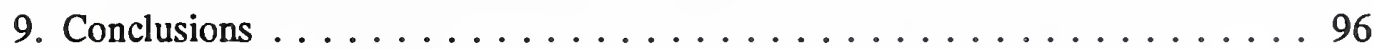

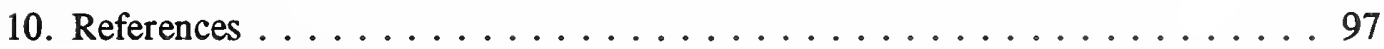




\section{List of Figures}

Figure 1. Schematic diagram of GMAW. The basic components of the welding torch are the power source, electrode feeder, electrode supply, contact tube, and gas nozzle ............... 2

Figure 2. Terminology and primary variables GMAW. . . . . . . . . . 4

Figure 3. The steady-state relationship developed by Lesnewich [4] for the GMAW primary input variables WFS, arc current, and electrode extension. The constants $a$ and $b$ are functions of electrode and shielding gas compositions. Typical values, $\mathrm{a}=0.367 \mathrm{~mm} /(\mathrm{s} \cdot \mathrm{A})$ and $\mathrm{b}=4.79 \times 10^{-5} /\left(\mathrm{s} \cdot \mathrm{A}^{2}\right)$, were used for this plot.

Figure 4. Composite nature of the GMAW process voltage. The process voltage, measured from the contact tip to the workpiece, is the sum of several individual voltages. . . . . . . . . . . . . 8

Figure 5. Arc voltage drop as a function of arc length. Arc voltage drop increases linearly with arc length. The voltage drop extrapolated to zero arc length is the sum of the anode and cathode voltage

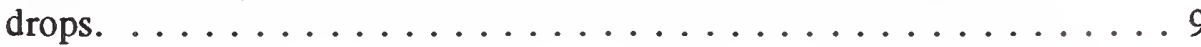

Figure 6. Arc voltage drop as a function of arc current. Arc voltage drop increases with arc current in the range associated with the spray transfer mode $\left(I_{A}>200 \mathrm{~A}\right) . \ldots \ldots \ldots 10 \ldots \ldots$

Figure 7. Electrical conductivity of Ar as a function of temperature. The conductivity reaches a plateau near $15000 \mathrm{~K}$ [15].

Figure 8. Definition of the process volume. Assuming the welding torch (heat source) is stationary and the workpiece moves, the unwelded joint flows into the left side of the volume, heat and filler flow in from the top, and the completed weld flows out the right.

Figure 9. Definitions of welding-process volume input and output. The ultimate goal of welding process control is to control the final outputs for which there are no in situ sensors. Process control in real time is limited to the primary and disturbing inputs and the intermediate outputs.

Figure 10. Block diagram representation of a process-control system, including the controller, actuator, and the process. Each component has a transfer function, $K$, defined as the block output, $O$, divided by the block input, $I$. The blocks are cascaded together to form the control system. . . . . . . . . . . . . . . . 13

Figure 11. Block diagram of open-loop process control. The open-loop control cannot correct for final output deviations caused by the disturbing inputs. 
Figure 12. Block diagram of closed-loop, feedforward control. A sensor detects disturbing inputs about to influence the process. That information is fed to the controller to make adjustments to the process input to minimize the effects on the process output.

Figure 13. Block diagram of closed-loop, feedback control block diagram based on sensing the final output, which operates the controller.

Figure 14. Closed-loop, feedback control block diagram based on intermediate-output sensing. A relationship between the intermediate output and the final output enables indirect control of

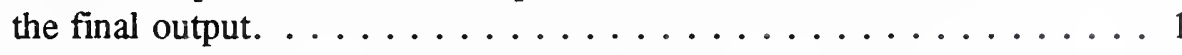

Figure 15. Overview of nomenclature for electromagnetic radiation and production mechanisms as a function of radiation wavelength. The electronic transitions of particles in the welding arc emit light in the visible and surrounding region [32]. . . . . . . . . .

Figure 16. The visible emission spectrum of an arc during the welding of steel with $98 \% \mathrm{Ar}+2 \% \mathrm{O}_{2}$ shielding gas using the GMAW process [33]

Figure 17. Energy level diagram showing levels and transitions for an atom, ion, and electron. $U_{0}$ is the ground state while $U_{1}$, $U_{2} \ldots$ are higher positive energy levels of atoms and ions. $U_{i}$ is the ionization energy. . . . . . . . . . . .

Figure 18. Radiated arc power in the wavelength range of 200 to $3500 \mathrm{~nm}$ as a function of temperature for a 200-A, 6-mm GTAW arc in Ar. The welding arc can be reasonably approximated as being optically thin [31]. . . . . . . . . . . . . . . . 23

Figure 19. The change in FeI- and FeII-line intensity with current during GTAW found by Kim [37]. Welding was conducted on AISI 304 stainless steel using Ar shielding gas.

Figure 20. The water-cooled coil used by Ghent et al. [38] to collect arc radiation as a function of arc length and current.

Figure 21. Arc radiation power collected in water-cooled coil as a function of arc length and current. Power in the coil increases approximately linearly with both current and length [38]. . . . . . . . . .

Figure 22. Source/detector configuration. $L_{S}$ is the radiance of the source with area $d A_{S} ; d \Phi$ is the incremental intensity incident on a section of a planar detector with area $d A_{D} ; \theta_{S}$ and $\theta_{D}$ are the angles that the source and detector, respectively, make with the line-of-sight axis; and $R_{D}$ is the distance between the source and detector. 
Figure 23. Welding equipment and instrumentation diagram. The welding equipment included a SCR-type power source, a transistorized current regulator, a capstan wire feeder, and a welding torch. The instrumentation included a data acquisition computer, Halleffect current sensor, WFS sensor, voltage sensor, and photodiode arc light detector. Both the current regulator and wire feeder were controlled by the computer. . . . . . . . . . . 28

Figure 24. Magnitude and phase-angle frequency-response curves of the current regulator. The regulator was capable of tracking the input signal at welding current frequencies up to $500 \mathrm{~Hz}$. . . . . . . 29

Figure 25. Magnitude and phase-angle frequency-response curves of the wire feeder. The wire feeder was capable of tracking the input signal at WFS frequencies up to $7 \mathrm{~Hz}$. . . . . . . . . . . . . . 29

Figure 26. Spectral response of the photodiode (detector) used to measure arc-light intensity. Note that the intensity scale is in relative units. The response of the detector was modified by placing optical filters in front of the detector. . . . . . . . . . . 30

Figure 27. Diagram of high-speed-video and laser-illumination system. Images were analyzed to extract CTWD, electrode extension, and arc length. The video-image collection was synchronized with the sensor data via an electronic link between the video recorder/player and the data-acquisition computer. . . . . . . . . 31

Figure 28. The source/detector geometric configuration. The center of the detector relative to the weld pool is given by the line-of-sight vector $\mathbf{r}$ and the plane of the detector has a normal vector $\mathbf{n}$. . . . . 37

Figure 29. The arc and blackbody spectra and the detector-response profile. With ND filtering, the response of the detector is a maximum in the near-infrared and extends beyond both ends of the available arc spectrum. Note that the traces are not scaled to each other; only the change in relative magnitude within each trace is represented.

Figure 30. Blue-band pass (BBP) optical-filter-transmission profile and arc and blackbody spectra. The filter attenuates the blackbody radiation and transmits a wide range of arc radiation. The arc spectrum in the passband consists primarily of Fe lines. The arc spectrum is from Peterson [33]. . . . . . . . . . . . . 40

Figure 31. Filter-transmission profile for a 430-nm filter with a half-power band width of $10 \mathrm{~nm}$ and the associated arc spectrum. Except for the lines noted, the arc spectrum consists of Fe lines. The arc spectrum is from Peterson [33]. . . . . . . . . . . . . 40

Figure 32. A 694-nm optical-filter-transmission profile and arc spectrum. The arc spectrum consists primarily of the continuum with a single Ar line at $696.5 \mathrm{~nm}$. The arc is spectrum from Peterson [33]. 
Figure 33. A 467-nm optical filter transmission profile and arc spectrum. The arc spectrum consists primarily of the continuum with an Mn line at the longer wavelengths. The arc spectrum is from Peterson [33]. . . . . . . . . . . . . . . . . . .

Figure 34. Video images for different welding conditions processed to obtain the arc length. The arc length is defined as the difference between the CTWD and the electrode extension.

Figure 35. Time traces for the process inputs during a weld. The current is modulated with a triangular waveform at $25 \mathrm{~Hz}$ about a mean level. The WFS is incremented as the weld progresses while the CTWD is constant.

Figure 36. Time traces for the process-output parameters. The electrode extension increases and the arc length decreases in response to increases in WFS. The mean and modulation amplitude of the arc-light-detector voltage decrease with arc length.

Figure 37. A section of the waveforms shown in figures 35 and 36 . The WFS is $123 \mathrm{~mm} / \mathrm{s}$, the CTWD is $20 \mathrm{~mm}$, and the arc length is $9 \mathrm{~mm}$. The current trace shows the triangular waveform of the input reference; the process voltage and detector voltage show similar behavior.

Figure 38. Detector voltage versus arc length for several values of current. The detector voltage (arc-light intensity) increases linearly with arc length, and the slope increases with increasing current.

Figure 39. Arc current versus detector voltage for several arc lengths. The detector voltage increases with current at a fixed arc length.

Figure 40. Current and arc-light-detector voltage response to an abrupt stop in welding at $t=0 \mathrm{~ms}$. The detector voltage after arc extinction $(0.26 \mathrm{~V})$ is attributed to weld-pool and electrode-tip radiation and is approximately 5 percent of the radiation when the $\operatorname{arc}$ is on. . . . . . . . . . . . . . . . . . 50

Figure 41. Example of correlation analysis results: (a) measured arc length versus estimated arc length illustrating data scatter and the correlation parameter $R_{c}$. (b) histogram of the error (difference) between the actual and estimated arc length for each point in the data set showing a normal distribution with a standard

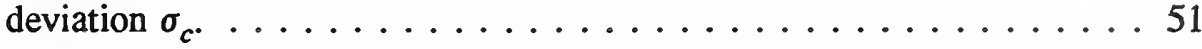

Figure 42. Detector voltage, correlation parameter, and error standard deviation as a function of the source/detector angle $\theta_{S}$ from welding using the BBP optical filter, $98 \% \mathrm{Ar}+2 \% \mathrm{O}_{2}$ shielding gas, $\theta_{D}=0^{\circ}$, and $R_{D}=400 \mathrm{~mm}$. Results indicate $\theta_{S}$ should be as close to $90^{\circ}$ as possible to obtain the best estimate of arc length. 
Figure 43. Detector voltage, correlation parameter, and error standard deviation as a function of the detector orientation angle $\theta_{D}$ from welding using the BBP optical filter, $98 \% \mathrm{Ar}+2 \% \mathrm{O}_{2}$ shielding gas, $\theta_{S}=85^{\circ}$, and $R_{D}=400 \mathrm{~mm}$. Results indicate that $\theta_{D}$ should be as close to $0^{\circ}$ as possible to obtain the best estimate of arc length. ........................ 54

Figure 44. Detector voltage, correlation parameter, and error standard deviation as a function of the detector distance $R_{D}$ from welding using the BBP optical filter, $98 \% \mathrm{Ar}+2 \% \mathrm{O}_{2}$ shielding gas, $\theta_{S}=85^{\circ}$, and $\theta_{D}=0^{\circ}$. Results indicate that the detector can operate over a range of distances if its output-voltage range is approximately equal to the $\mathrm{ADC}$ range.

Figure 45. Estimated versus measured arc length and error histogram resulting from using the 467-nm optical filter and $95 \% \mathrm{Ar}$ $+5 \% \mathrm{CO}_{2}$ shielding gas. . . . . . . . . . . . . . 5

Figure 46. Estimated versus measured arc length and error histogram resulting from using the $467-\mathrm{nm}$ optical filter and $98 \% \mathrm{Ar}$ $+2 \% \mathrm{O}_{2}$ shielding gas. . . . . . . . . . . . . . 57

Figure 47. Estimated versus measured arc length and error histogram resulting from using the $430-\mathrm{nm}$ optical filter and $95 \% \mathrm{Ar}$ $+5 \% \mathrm{CO}_{2}$ shielding gas. ................. 58

Figure 48. Estimated versus measured arc length and error histogram resulting from using the $430-\mathrm{nm}$ optical filter and $98 \% \mathrm{Ar}$ $+2 \% \mathrm{O}_{2}$ shielding gas. ..................... 58

Figure 49. Estimated versus measured arc length and error histogram resulting from using the 694-nm optical filter and $95 \% \mathrm{Ar}$ $+5 \% \mathrm{CO}_{2}$ shielding gas. .................. 59

Figure 50. Estimated versus measured arc length and error histogram resulting from using the 694-nm optical filter and $98 \% \mathrm{Ar}$ $+2 \% \mathrm{O}_{2}$ shielding gas.

Figure 51. Estimated versus measured arc length and error histogram resulting from using the ND optical filter and $95 \% \mathrm{Ar}$

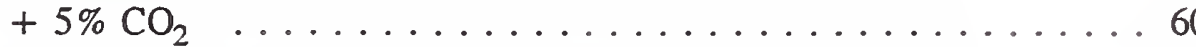

Figure 52. Estimated versus measured arc length and error histogram resulting from using the ND optical filter and $98 \% \mathrm{Ar}$ $+2 \% \mathrm{O}_{2}$ shielding gas. . . . . . . . . . . . . . . 60

Figure 53. Estimated versus measured arc length and error histogram resulting from using the BBP optical filter and $95 \% \mathrm{Ar}$ $+5 \% \mathrm{CO}_{2}$ shielding gas.

Figure 54. Estimated versus measured arc length and error histogram resulting from using the $\mathrm{BBP}$ optical filter and $98 \% \mathrm{Ar}$ $+2 \% \mathrm{O}_{2}$ shielding gas. ................... 6 
Figure 55. Summary of the correlation coefficient, $R_{c}$, and standard deviation, $\sigma_{c}$, for each optical filter and shielding gas. Higher values of $R_{c}$ and lower values of $\sigma_{c}$ indicate better arc length estimation.

Figure 56. Response of arc length to a step increase in arc current for determining the static and dynamic characteristics of the welding process. The WFS was $123 \mathrm{~mm} / \mathrm{s}$. The solid curve shows the mathematical model. . . . . . . . . . . . . . 67

Figure 57. Block diagram of the arc-length control system. . . . . . . . . 68

Figure 58. Closed-loop response of the control system to step increase in arc-length setpoint from 6 to $9 \mathrm{~mm}$. The WFS was $135 \mathrm{~mm} / \mathrm{s}$. The solid curve shows the mathematical model.

Figure 59. Closed-loop response of the control system to a disturbing input in the form of a step increase in WFS from 140 to $170 \mathrm{~mm} / \mathrm{s}$. The solid curve shows the mathematical model. . . . . . . . . . . 70

Figure 60. Response of a typical CV power source to a disturbing input in the form of a step increase in WFS from 140 to $170 \mathrm{~mm} / \mathrm{s}$.

Figure 61. Static-transfer-mode map developed for determining the droplet transfer mode based on average current and voltage (from Heald [67]). . . . . . . . . . . . . . . . . . 73

Figure 62. Video-image processing used to obtain the reference or actual droplet-transfer frequency. The change in electrode extension, as the droplet detaches, produces a sawtooth waveform from which the transfer frequency can be readily obtained. . . . . . . . 75

Figure 63. Correlation of droplet detachment with arc-light-detector voltage. The sawtooth waveform of the detector-voltage signal is similar to that of the electrode-extension signal but shifted in phase by $180^{\circ} . \ldots \ldots \ldots \ldots \ldots \ldots \ldots . \ldots \ldots$

Figure 64. Time traces of the current, process voltage, detector voltage, and electrode extension for a spray-transfer weld using the current regulator. Droplet-detachment features are present in the detector-voltage, electrode-extension, and process-voltage traces but not in the current trace. Optical filter: BBP. Welding conditions: $98 \% \mathrm{Ar}+2 \% \mathrm{O}_{2}, 255 \mathrm{~A}, 130 \mathrm{~mm} / \mathrm{s} . \ldots \ldots . \ldots 80$

Figure 65. PSD plots for the weld in figure 64. The electrode-extension, and detector-voltage spectra show the actual droplet-transfer frequency of $222 \mathrm{~Hz}$. The process voltage and current spectra show peaks at the droplet transfer frequency and at the power-source line frequencies of 120 (maximum), 180, and

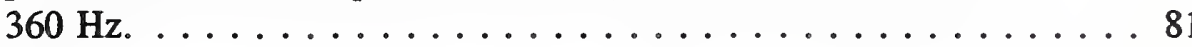


Figure 66. Time traces of the current, process voltage, detector voltage, and electrode extension for a streaming-spray-transfer weld made by using the current regulator. The detector voltage and electrode extension show the droplet-detachment signature. Neither the process voltage nor the current show dropletdetachment features. Optical filter: $430 \mathrm{~nm}$. Welding conditions: $98 \% \mathrm{Ar}+2 \% \mathrm{O}_{2}, 320 \mathrm{~A}, 180 \mathrm{~mm} / \mathrm{s}$.

Figure 67. PSD plots for the weld in figure 66. The electrode-extension and detector-voltage spectra show the actual droplet-transfer frequency of $397 \mathrm{~Hz}$. Peaks in the current and voltage spectra are related to the power-source line frequency $(360 \mathrm{~Hz}$ maximum), and small peaks occur in both spectra at the transfer frequency. ...................... 83

Figure 68. Time traces of the current, process voltage, detector voltage, and electrode extension for a spray-transfer weld made by using the CV, SCR power source. The electrode-extension and detector-voltage traces show the droplet-detachment signature. The current and process-voltage traces show droplet detachment confounded by the power-source ripple. Optical filter: $430 \mathrm{~nm}$. Welding conditions: $98 \% \mathrm{Ar}+2 \% \mathrm{O}_{2}, 32.5 \mathrm{~V}, 144 \mathrm{~mm} / \mathrm{s} . \ldots . .8$

Figure 69. PSD plots for the weld in figure 68. The electrode-extension and detector-voltage spectra show the actual droplet-transfer frequency of $272 \mathrm{~Hz}$, with a smaller peak at $360 \mathrm{~Hz}$ in the detector-voltage spectrum. The current and voltage spectra contain power-source line frequency peaks (maximum at $360 \mathrm{~Hz}$ ) and a large number of significant peaks related to the SCR power-source characteristics.

Figure 70. The normalized detector voltage ratio $\xi_{D}$ for each combination of shielding gas and optical filter. For both shielding gases, the maximum $\xi_{D}$ occurred when the 430 -nm filter was used, indicating that filter offers the best droplet-frequency detection performance. Other welding conditions: $255 \mathrm{~A}, 130 \mathrm{~mm} / \mathrm{s}$.

Figure 71. Droplet-transfer frequency and electrode extension as functions of current for two values of constant WFS. . . . . . . . . . 990

Figure 72. Droplet-transfer frequency and electrode extension as a function of WFS at two values of current. . . . . . . . . . . . . 91

Figure 73. Droplet-transfer frequency and WFS as a function of current for two values of constant electrode extension. The WFS was changed with the current to obtain constant electrode extension. . . . 92

Figure 74. Block diagram of droplet-frequency control. . . . . . . . . . . . . 93

Figure 75. Response of the transfer-frequency controller to a step increase in frequency setpoint with only the current actuated. The WFS was constant at $144 \mathrm{~mm} / \mathrm{s}$. 
Figure 76. Response of the droplet-transfer-frequency controller to a step increase in frequency setpoint with both current and WFS actuated. 


\section{List of Tables}

Table 1. Optimum geometric configuration relative to the arc for arclength estimation. ........................ 56

Table 2. Summary of coefficients for the arc-length-sensor model [eq (43)], correlation parameter, and standard deviation for each combination of shielding gas and optical filter using the detector location and orientation shown in table $1 \ldots \ldots . . \ldots 62$ 


\section{List of Symbols and Acronyms}

a

ADC

AISI

AVC

$A_{A}$

$A_{D}$

$A_{e}$

$A_{S}$

b

BBA

BBP

BBE

BFA

c

$\mathrm{C}, \mathrm{C}_{i}$

CTWD

CV

$d_{d}$

$d_{e}$

DAC

DCEN

DCEP

$E_{A}$

$E_{\text {anode }}$

$E_{\text {cathode }}$

$E_{\text {column }}$

$E_{\text {contact }}$

$E_{D}$

$E_{e}$

$E_{0}$

$E_{P}$

$f_{d}$

$f_{D}$

$F_{R}$

FBE

FFA

FFE constant

analog-to-digital converter

American Iron and Steel Institute

automatic voltage control

arc-column cross-sectional area $\left(\mathrm{mm}^{2}\right)$

detector area $\left(\mathrm{mm}^{2}\right)$

electrode cross-sectional area $\left(\mathrm{mm}^{2}\right)$

source area $\left(\mathrm{mm}^{2}\right)$

constant

bound-bound absorption

blue-band pass

bound-bound emission

bound-free absorption

speed of light

constants

contact-tube-to-work distance (mm)

constant voltage

droplet diameter $(\mathrm{mm})$

electrode diameter $(\mathrm{mm})$

digital-to-analog converter

direct-current electrode negative

direct-current electrode positive

arc voltage $(\mathrm{V})$

arc-anode voltage (V)

arc-cathode voltage (V)

arc-column voltage (V)

contact-tube/electrode-interface voltage (V)

arc-light-detector voltage (V)

electrode-extension voltage (V)

arc-anode + arc-cathode voltage (V)

welding-process voltage (V)

measured droplet frequency $(\mathrm{Hz})$

desired droplet frequency $(\mathrm{Hz})$

frequency resolution $(\mathrm{Hz})$

free-bound emission

free-free absorption

free-free emission 


\begin{tabular}{|c|c|}
\hline $\begin{array}{l}G_{W} \\
\text { GMAW } \\
\text { GTAW }\end{array}$ & $\begin{array}{l}\text { welding-process gain } \\
\text { gas-metal-arc welding } \\
\text { gas-tungsten-arc welding }\end{array}$ \\
\hline$\stackrel{\hbar}{H_{W}}$ & $\begin{array}{l}\text { Planck's constant } \\
\text { welding heat input }(\mathrm{J} / \mathrm{mm})\end{array}$ \\
\hline $\begin{array}{l}I \\
I_{A} \\
I_{C} \\
I_{F F} \\
I_{W}\end{array}$ & $\begin{array}{l}\text { input } \\
\text { arc current (A) } \\
\text { controller output current (A) } \\
\text { feedforward current (A) } \\
\text { welding current (A) }\end{array}$ \\
\hline $\begin{array}{l}\mathrm{k} \\
k \\
K \\
K_{A} \\
K_{C} \\
K_{D} \\
K_{I} \\
K_{P} \\
K_{S} \\
K_{W} \\
K_{\rho}\end{array}$ & $\begin{array}{l}\text { constant } \\
\text { sample period } \\
\text { transfer function } \\
\text { actuator transfer function } \\
\text { controller transfer function } \\
\text { controller derivative gain } \\
\text { controller integral gain } \\
\text { controller proportional gain } \\
\text { sensor transfer function } \\
\text { welding-process transfer function } \\
\text { process transfer function }\end{array}$ \\
\hline $\begin{array}{l}L \\
\Delta \ell \\
\ell_{0} \\
\ell_{A} \\
L_{A} \\
\ell_{D} \\
\ell_{e} \\
L_{S}\end{array}$ & $\begin{array}{l}\text { light signal } \\
\text { arc-length error }(\mathrm{mm}) \\
\text { initial arc length }(\mathrm{mm}) \\
\text { arc length }(\mathrm{mm}) \\
\text { volume-source radiant power }\left[\mathrm{W} /\left(\mathrm{mm}^{3} \cdot \mathrm{sr}\right)\right] \\
\text { desired arc length }(\mathrm{mm}) \\
\text { electrode extension }(\mathrm{mm}) \\
\text { planar-source radiance }\left[\mathrm{W} /\left(\mathrm{mm}^{2} \cdot \mathrm{sr}\right)\right]\end{array}$ \\
\hline$m$ & detector-voltage/arc-length slope \\
\hline $\begin{array}{l}\mathbf{n} \\
\mathrm{ND}\end{array}$ & $\begin{array}{l}\text { vector normal to the detector } \\
\text { neutral density }\end{array}$ \\
\hline$O$ & output \\
\hline $\begin{array}{l}P_{A} \\
P_{K} \\
P_{L} \\
P_{V} \\
P_{W} \\
\text { PID } \\
\text { PSD }\end{array}$ & $\begin{array}{l}\text { arc power (W) } \\
\text { arc power lost by conduction (W) } \\
\text { arc power lost by radiation }(W) \\
\text { arc power lost by convection }(W) \\
\text { welding-power input }(W) \\
\text { proportional-integral derivative } \\
\text { power spectral density }\end{array}$ \\
\hline
\end{tabular}




$\begin{array}{ll}r & \text { line-of-sight vector } \\ R_{c} & \text { regression-correlation parameter } \\ R_{D} & \text { source-to-detector distance (mm) } \\ S & \text { Laplace-transform variable } \\ S & \text { welding-travel speed (mm/s) } \\ \text { SCR } & \text { silicon-controller rectifier } \\ \text { std } & \text { standard deviation } \\ t & \text { time } \\ U_{i} & \text { atomic energy levels (eV) } \\ U_{I} & \text { ionization energy } \\ V_{A} & \text { arc volume (mm }{ }^{3} \text { ) } \\ \text { WFS } & \text { wire-feed speed (mm/s) } \\ \alpha, \alpha_{i} & \text { constants } \\ \beta & \text { fraction of arc power lost by radiation, constant } \\ \gamma & \text { arc-voltage/arc-length gradient } \\ \zeta_{i} & \text { constants } \\ \eta & \text { efficiency factor (dimensionless) } \\ \theta_{D} & \text { detector/line-of-sight axis angle } \\ \theta_{S} & \text { source/line-of-sight axis angle } \\ \theta_{Z} & \text { detector orientation angle } \\ \lambda & \text { wavelength (nm) } \\ \nu & \text { frequency of a photon (Hz) } \\ \nu_{m} & \text { electrode melting rate (mm/s) } \\ \xi_{D} & \text { normalized detector-voltage ratio (percent) } \\ \rho_{e} & \text { electrode resistivity } \\ \sigma_{A} & \text { arc-column conductivity (S) } \\ \sigma_{c} & \text { standard deviation (mm) } \\ \sigma_{e} & \text { effective electrode conductivity (S) } \\ \tau_{W} & \text { welding-process time constant (s) } \\ \Phi & \text { radiant power (W) } \\ \Omega_{D} & \text { detector solid angle (sr) } \\ & \\ & \end{array}$





\title{
Control of Gas-Metal-Arc Welding Using Arc-Light Sensing
}

\author{
R. Bruce Madigan, T. P. Quinn, and T. A. Siewert \\ National Institute of Standards and Technology \\ Boulder, CO 80303
}

In this development of closed-loop control of the gas-metal-arc welding process using light emitted by the arc, two process parameters were controlled in real time: arc length and droplet-transfer frequency. Increases in arc light with both arc length and arc current are explained by treating the arc as an optically thin, volume radiation source. An arc-length sensor and controllers to regulate arc length and to actuate welding current were developed. The transfer frequency depended on current, wire-feed speed, and electrode extension; the results led to the design of a transferfrequency controller.

Key words: arc length; arc-light intensity; automated welding; closedloop control; droplet frequency; gas-metal-arc welding (GMAW); modeling; optical spectrum; process control; radiation; sensor; welding parameters

\section{Introduction}

Arc welding has traditionally relied on the skill of the operator to apply and control the processes and to produce quality welds. Demands for increased productivity while maintaining or increasing quality, an increased reliance on statistical process control, and the desire to remove the operator from the welding environment have driven the automation of welding processes [1].

Arc welding is a complex process that includes many variables that affect weld quality and many subtle tasks (such as weld-joint tracking and weld-pool control) that a human operator accomplishes with relative ease. Removing the welder from proximity to the welding processes removes a sense of the process, which is difficult to regain or mimic with machines.

One of the major obstacles in the practical application of automatic controls is the difficulty in developing techniques for measuring process parameters in real time. The welding environment is harsh: intense electromagnetic radiation, high electric and magnetic fields, and molten-metal spatter and fumes have deleterious effects

NOTE: Any trade names used in this report are given solely to provide complete identification of the equipment used. Such identification of products neither constitutes nor implies endorsement of the products or their manufacturers. 
on delicate electronic sensors. Once robust sensors have been developed, the next challenge is interpretation of sensory data to extract meaningful information about the process. Then control systems must be designed, tested, and applied to improve the welding process.

\subsection{Fundamentals of Gas-Metal-Arc Welding}

In gas-metal-arc welding (GMAW), schematically represented in figure 1 , an arc is maintained between a solid, consumable electrode and the base-metal components being joined [2]. The arc melts the base metal and the electrode, producing a molten weld pool that coalesces to form the completed weld. A continuous electrode is fed into the arc at a rate that matches the melting rate. Power for the arc is transferred to the electrode through a contact tube mounted to the body of the welding torch. Molten droplets formed on the end of the electrode transfer to the weld pool, becoming filler metal for the weld joint. The metal-transfer modes-short-circuiting, globular, and spray-are determined by the welding parameters.

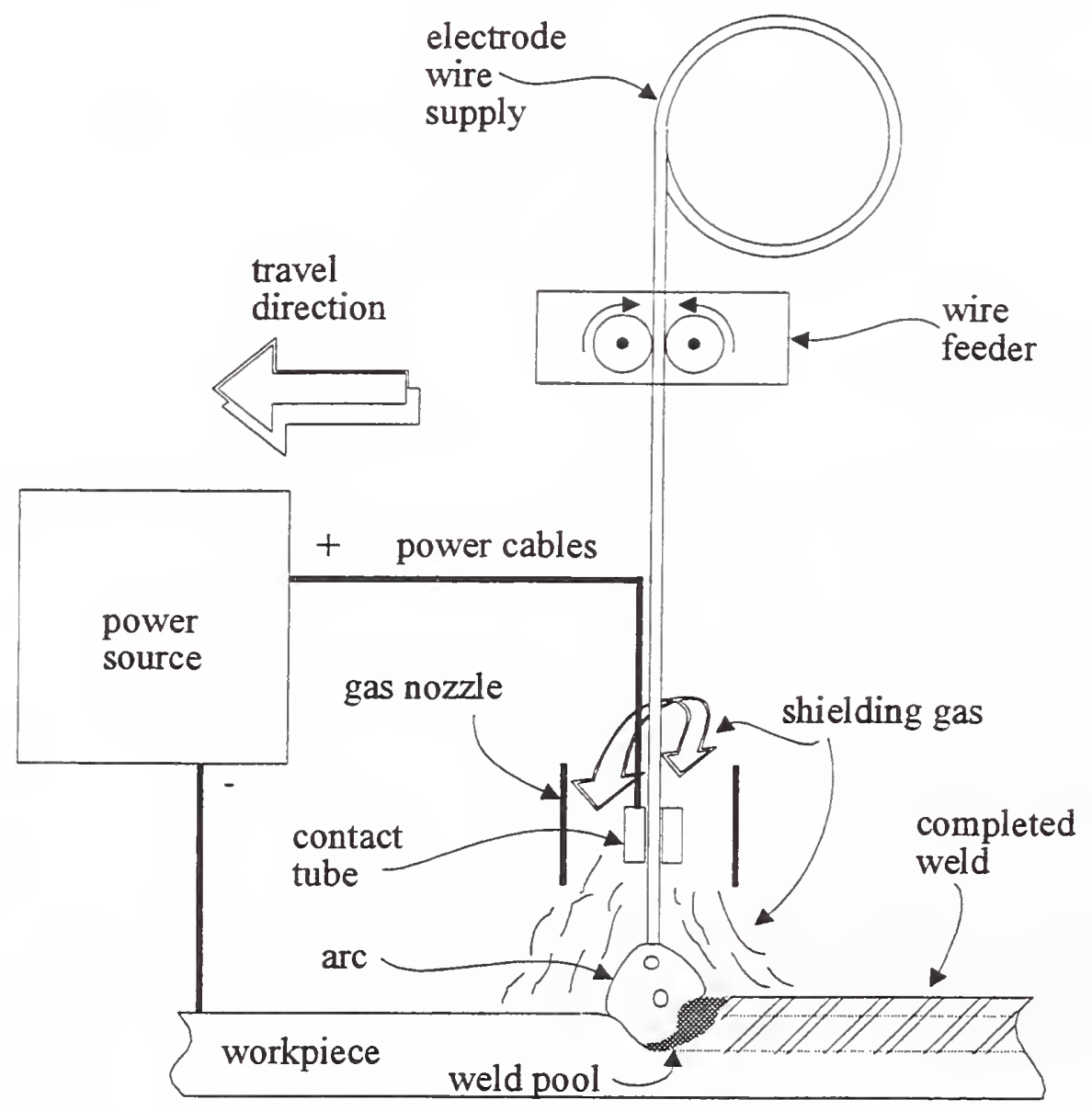

Figure 1. Schematic diagram of GMAW. The basic components of the welding torch are the power source, electrode feeder, electrode supply, contact tube, and gas nozzle. 
The atmosphere is displaced from the welding area by an inert or reactive shielding gas flowing from the gas nozzle. Motion of the torch relative to the joint completes the weld.

\subsection{Objective and Approach}

The objective of this work was to demonstrate the real-time, closed-loop control of two aspects of GMAW: arc length and droplet-transfer frequency.

The approach relied on correlating experimental data with analytical and empirical models that characterize the process. An array of transducers provided raw data from the process that was sampled by a computer. The raw data were interpreted through the models coded into the computer software. The process information extracted acted as the sensory input to a controller, which was also coded into the software. Thus, by manipulating the process inputs, the controller regulated the welding.

\section{Characteristics of the Process and Process Control}

The principal GMAW-process variables and a mathematical description of the process are the foundations for design of automated process control. The concepts of closed-loop control are reviewed with respect to this application, and the practical aspects of applying control technology to welding are discussed.

\subsection{Process Variables}

The primary input variables adjusted for application of the GMAW process are power-source current or voltage, the wire-feed speed (WFS), contact-tube-to-work distance (CTWD), and welding-travel speed (see fig. 2). Arc length and electrode extension are determined by adjustment of the WFS and power-source current or voltage. Shielding-gas, electrode and workpiece composition, and travel speed are also primary input variables; typically, they are fixed for a given application.

Usually, GMAW is conducted by using direct current with the electrode positive (DCEP) and the workpiece negative. Operating the process with the opposite polarity (DCEN) leads to an unstable arc and excessive spatter [3]. Both constantcurrent and constant-voltage power sources are used. The design of the power source determines the static and dynamic response characteristics of the power source and influences the process. Modern power sources are capable of maintaining the desired arc current or process voltage within tight tolerances.

The proper electrode extension has been determined experimentally; it varies with shielding-gas and electrode compositions and electrode diameter [4]. Electrode extension is determined primarily by the WFS and welding current. Joule heating in the electrode, which varies with electrode extension, provides a portion of the heat required to melt the electrode. Therefore, in addition to the current, the electrode extension plays an important role in determining the melting rate and the transfer mode of the electrode. 


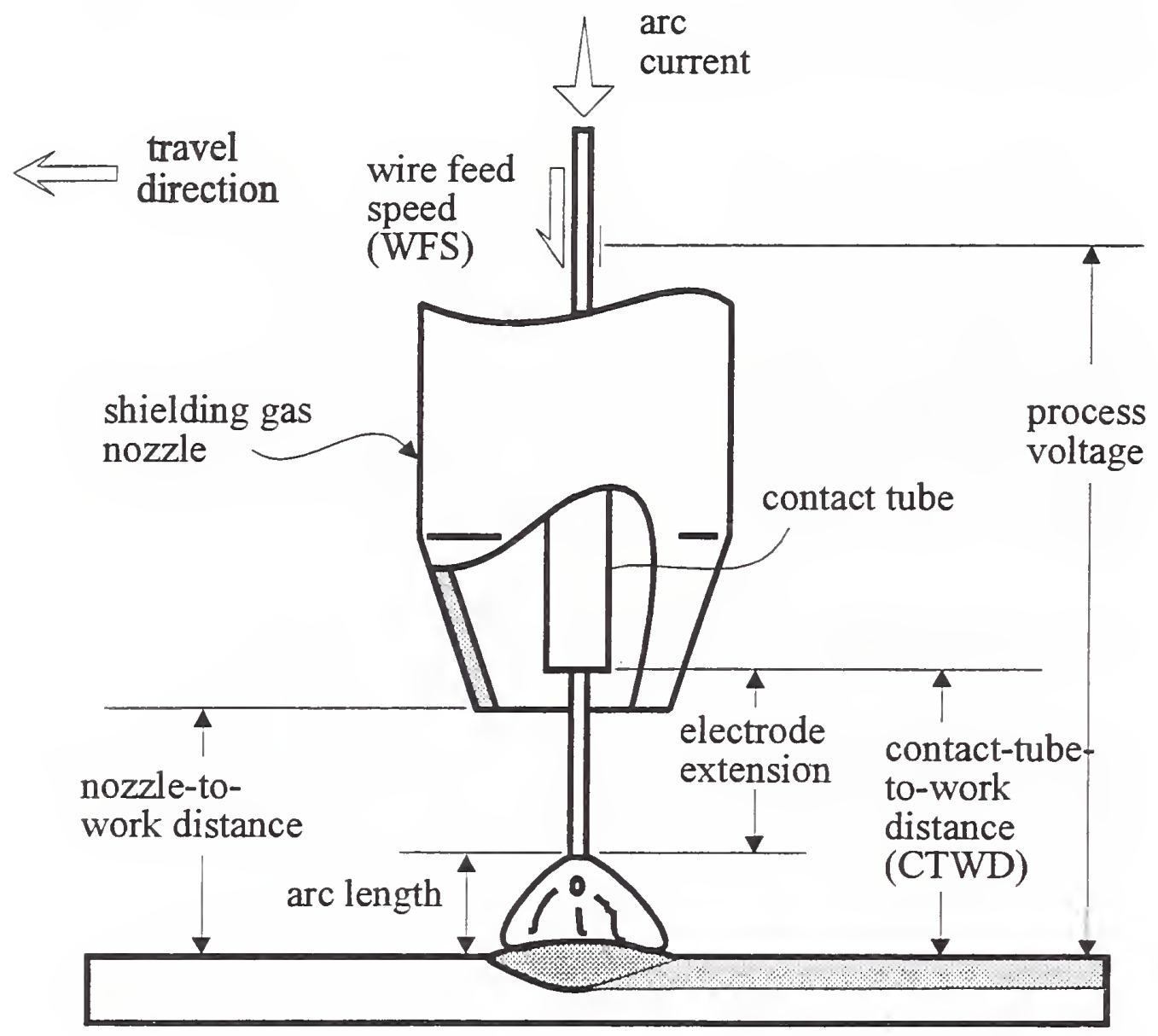

Figure 2. Terminology and primary variables of GMAW.

Under steady-state conditions, the WFS is equal to the melting rate of the electrode, and the electrode melting rate is primarily determined by the arc current. The WFS is usually held constant during welding; however, it can be adjusted to achieve a range of filler-metal-deposition rates.

Arc length determines the current and the arc-pressure distribution on the weld pool, which, in turn, determines the size and shape of the weld-fusion zone [5-7]. With insufficient arc length, the electrode will contact or short to the weld pool, resulting in reduced base-metal melting, a high and narrow weld deposit, variation in heat input, and increased chances of defect formation. Excessive arc length causes a flat, shallow deposit; causes the arc to wander; increases spatter; and may cause porosity from air aspirated into the shielding gas. To obtain consistent weld quality, it is essential that arc length be controlled. 


\subsection{Transfer Mode}

The mode of metal transfer from the electrode tip to the weld pool in GMAW depends on many factors, including arc current, process voltage, WFS, electrode extension, shielding-gas composition, and electrode composition. The transfer mode directly determines process stability and, thus, weld quality by influencing the width, penetration, solidification, and heat flow of the weld pool [8]. Therefore, to obtain consistent weld quality, the desired transfer mode must be maintained. The useful transfer modes in GMAW are short-circuiting, globular, and spray [9].

The short-circuiting transfer mode occurs at low welding current and a high WFS. It is characterized by periodic arc extinction as the electrode contacts or shorts to the weld pool and arc re-ignition as the short is cleared. Metal is transferred from the electrode each time it contacts the weld pool. Heat input is limited by the arc extinction and by the expulsion of molten metal in the form of spatter. Because its heat input is limited, short-circuiting transfer is used for joining thin sheet metal and for welding out-of-position, vertical, and overhead joints. The large variations in current and voltage due to the shorting impart a readily detectable transfer-mode signature in the current and voltage signals.

Globular transfer develops at a longer arc (lower WFS) than that of shortcircuiting transfer. The diameters of the molten droplets grow to several times that of the electrode before detaching. The droplet detaches when the combination of gravitational force and Lorentz force overcomes the surface-tension force holding it to the electrode. The globules tend to be randomly directed with respect to the electrode axis and periodically short to the weld pool, causing spatter. Globular transfer is unstable and is not widely used. Like the short-circuit-transfer mode, the large variations in arc length and electrode extension during the globular-transfer mode are readily observed in the current and voltage signals.

At higher current and WFS, the transfer mode changes to spray. The higher current imposes a significant Lorentz "pinching" force on the droplet that dominates the gravitational force. Also, spray transfer requires the use of an active shielding gas that reduces the surface tension of the droplet. The combination of increased Lorentz force and reduced surface tension produces small droplets that are directed axially across the arc to the weld pool. Spray transfer can be subdivided into drop-spray and streaming-spray modes, differentiated by the size of the droplets. The drop-spray mode occurs when the droplets are about the same diameter as the electrode wire. Increased WFS and current produces the streaming-spray transfer mode in which the droplets are smaller in diameter than the electrode wire. The spray-transfer mode is stable; it is used for welding thick sections where high heat input and deposition rate are beneficial. The small droplet size makes it difficult to determine droplet detachment from the voltage and current signals. The spray-transfer mode is central to this study.

\subsection{Mathematical Modeling}

Analytical equations have been developed to characterize the GMAW process. Theoretical analysis provides insight into process behavior and forms the basis for developing process-control techniques. 


\subsubsection{Heat Input}

The energy or power input for melting and formation of the weld can be expressed as [10]

$$
P_{W}=\eta E_{P} I_{A}
$$

where $P_{W}$ is the welding-power input, $E_{P}$ is the welding-process voltage, $I_{A}$ is the $\operatorname{arc}$ current, and $\eta$ is a dimensionless efficiency factor ranging from 0 to 1 that accounts for losses in the welding circuit. A convenient measure of the energy deposited in the weld can be expressed in terms of energy per unit length or

$$
H_{W}=\frac{P_{w}}{S}=\eta \frac{E_{P} I_{A}}{S},
$$

where $H_{W}$ is the heat input per unit length of the weld and $S$ is the welding-travel speed.

As indicated in eqs (1) and (2), only a fraction of the total power available from the power source, $\eta$, is available to form the weld. Power is also lost through dissipation within electrical conductors and contact interfaces and thermal losses associated with conduction, convection, and radiation from the arc, workpiece, electrode, and torch. Most of the power loss occurs through the arc, and it increases with both arc length and current.

\subsubsection{Electrode Melting Rate}

Under equilibrium conditions, the WFS equals the melting rate of the electrode. An empirical, steady-state expression for the relationship among the GMAW process variables is

$$
\mathrm{WFS}=\nu_{m}=\mathrm{a} I_{A}+\mathrm{b} \ell_{e} I_{A}^{2},
$$

where $\nu_{m}$ is the electrode melting rate, $\ell_{e}$ is the electrode extension, $I_{A}$ is the $\operatorname{arc}$ current, $a$ is a constant related to arc voltage, and $b$ is a constant related to resistive heating in the electrode extension $[4,11]$. A plot of eq (3), provided in figure 3 , shows that a wide range of WFS, current, and electrode-extension values can satisfy the steady-state relationship.

Neglecting any depression of the weld-pool surface due to arc pressure, the CTWD can be expressed as the sum of the electrode extension and arc length:

$$
\text { CTWD }=\ell_{e}+\ell_{A}
$$

where $\ell_{\mathrm{A}}$ is the arc length.

\subsubsection{Process Voltage}

An expression for voltage does not appear in the process-governing relationship, eq (3). The voltage $E_{P}$ in eqs (1) and (2) is simply that potential that must be developed by the power source to drive the current $I_{A}$ in the welding circuit. The value of $E_{P}$ is commonly measured between the output terminals of the power source or between the point where the power conductors attach to the contact tube and the base metal. Here, it is called welding-process voltage to distinguish it from the true arc column voltage. As shown in figure $4, E_{P}$ is the sum of distinct voltage-drop 


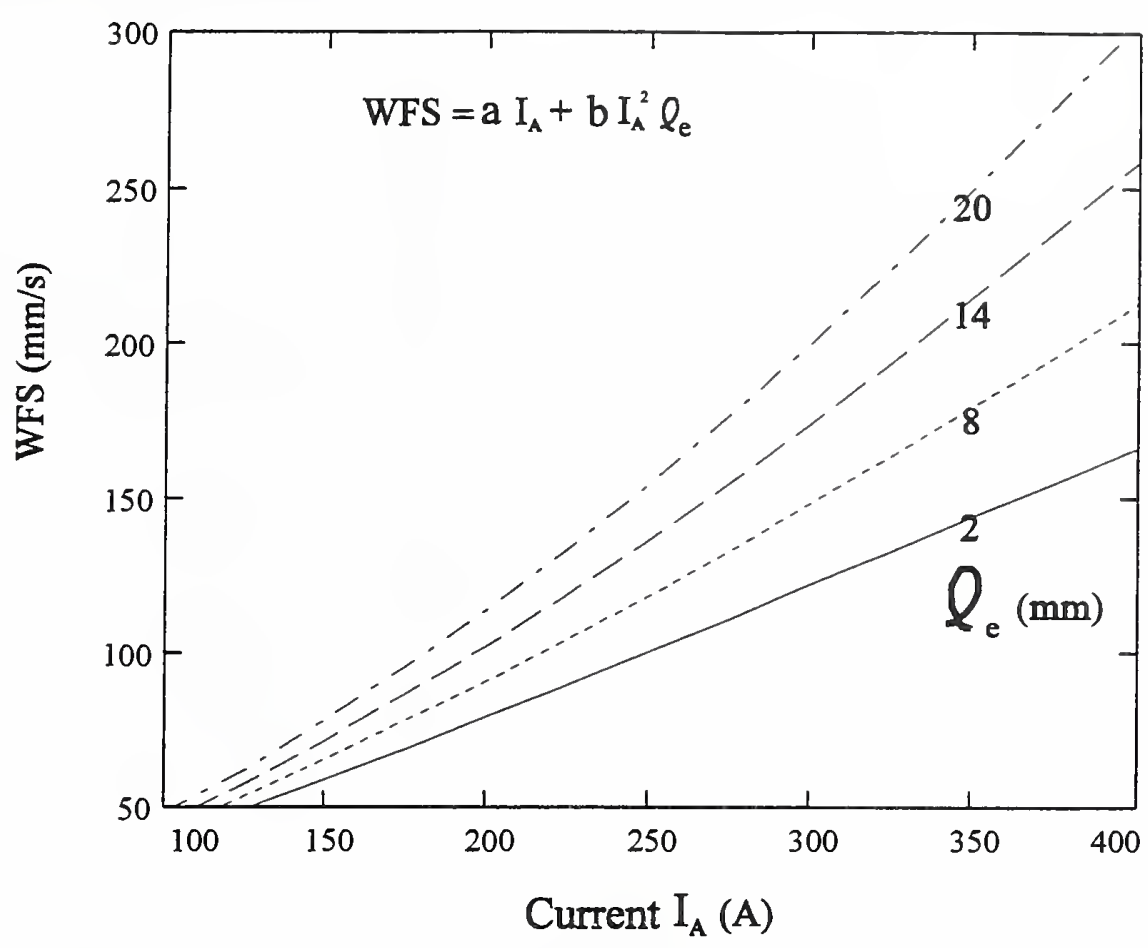

Figure 3. The steady-state relationship developed by Lesnewich [4] for the GMAW primary input variables WFS, arc current, and electrode extension. The constants $\mathrm{a}$ and $\mathrm{b}$ are functions of electrode and shielding gas compositions. Typical values, $a=0.367 \mathrm{~mm} /(\mathrm{s} \cdot \mathrm{A})$ and $\mathrm{b}=4.79$ $\times 10^{-5} /\left(\mathrm{s} \cdot \mathrm{A}^{2}\right)$, were used for this plot.

elements: those at the tube/electrode interface, $E_{\text {contact }} ;$ along the electrode extension, $E_{e}$; at the arc/cathode interface, $E_{\text {cathode; }}$; at the arc column, $E_{\text {column}}$; and at the arc/ anode interface, $E_{\text {anode }}[9]$. Thus, the process voltage drop $E_{p}$ can be expressed as

$$
E_{p}=E_{\text {contact }}+E_{e}+E_{\text {cathode }}+E_{\text {column }}+E_{\text {anode }} \text {. }
$$

The contact voltage drop $E_{\text {contact }}$ results from the sliding contact between the electrode and contact tube. As the contact tube wears, $E_{\text {contact }}$ can vary, leading to erratic current transfer"and process instability.

The voltage drop along the electrode extension $E_{e}$, which is primarily due to ohmic heating, can be obtained from Ohm's law [12,13]:

$$
E_{e}=I_{A} \frac{\ell_{e}}{\sigma_{e} A_{e}},
$$

where $A_{e}$ is the electrode cross-sectional area and $\sigma_{\mathrm{e}}$ is the effective electrode conductivity, which varies with temperature (and temperature varies with WFS).

Neither the arc-anode voltage drop $E_{\text {anode }}$ nor the cathode voltage drop $E_{\text {cathode }}$ is well understood, but some theories exist $[9,14]$. These voltage drops occur across small distances at electrode/arc-column and arc-column/weld-pool boundary layers. 


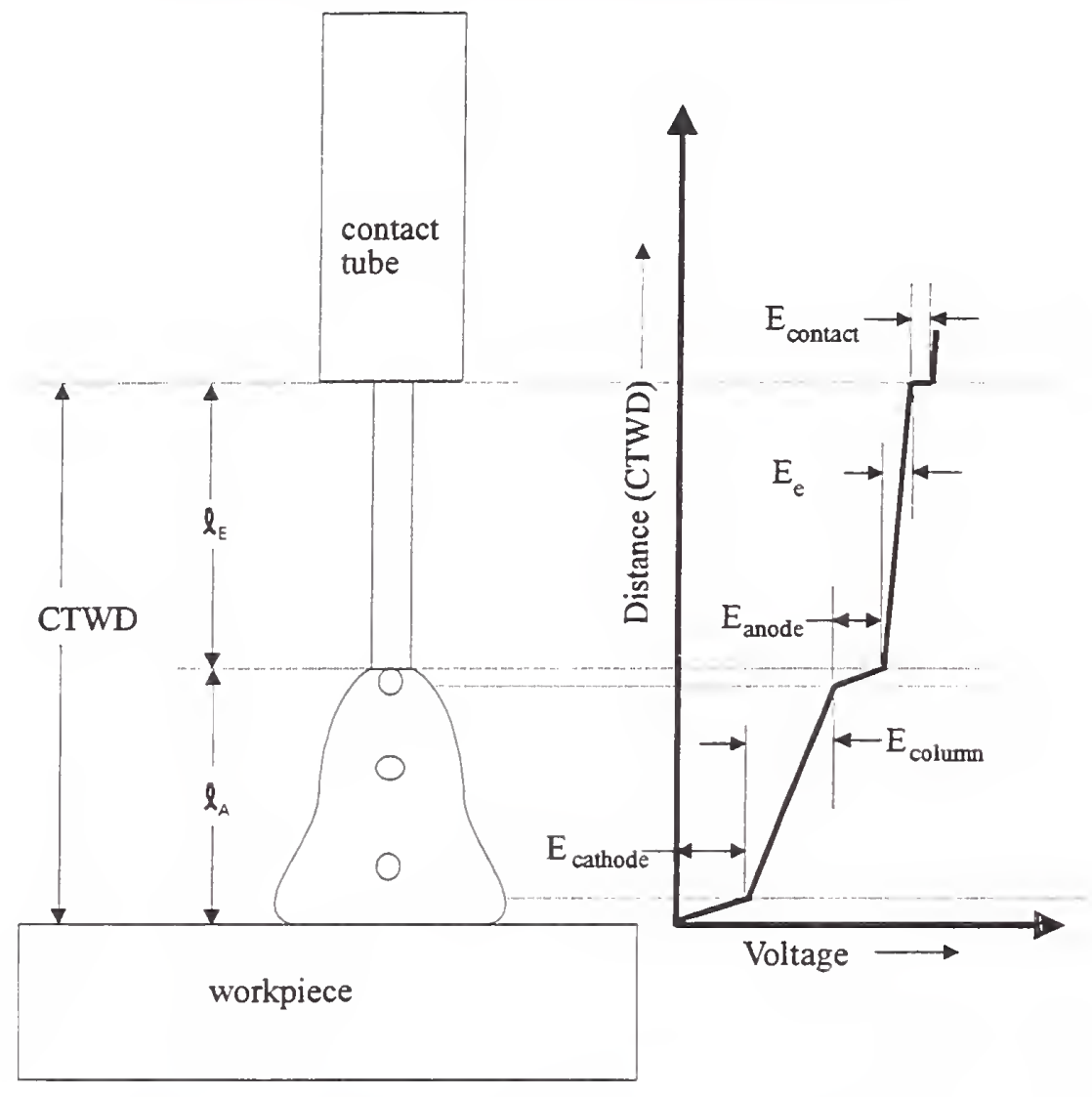

Figure 4. Composite nature of the GMAW process voltage. The process voltage, measured from the contact tube to the workpiece, is the sum of several voltages.

Both are a function of electrode and shielding-gas compositions and are considered constants for fixed compositions. Typically, the arc-anode and arc-cathode voltages drops are combined and expressed as

$$
E_{O}=E_{\text {anode }}+E_{\text {cathode }},
$$

where $E_{O}$ is the combined anode and cathode voltage drop.

In practice, it is difficult to separate the actual column voltage drop from the anode and cathode voltage drops. The term arc voltage drop usually refers to the sum of the column, anode, and cathode voltage drops:

$$
E_{A}=E_{O}+E_{\text {column }} .
$$

Experimentally, the arc voltage drop has been shown to change as a function of both arc current and arc length. At constant current, the arc voltage has been observed to vary linearly with length: 


$$
E_{A}=E_{O}+\frac{\Delta E_{A}}{\Delta \ell_{A}} \ell_{A}=E_{O}+\gamma \ell_{A},
$$

where $\gamma=\Delta E_{A} / \Delta \ell_{A}$ is the arc-voltage/arc-length gradient, which is primarily a function of the shielding-gas composition [9]. Equation (9) is shown graphically in figure 5. Under constant arc-length conditions, Goldman (cited in Lancaster [9]) developed the following relationship for arc voltage as a function of current

$$
E_{A}=\mathrm{C}_{0}+\mathrm{C}_{1} I_{A}=\frac{\mathrm{C}_{2}}{I_{A}},
$$

where $\mathrm{C}_{0}, \mathrm{C}_{1}$, and $\mathrm{C}_{2}$ are constants obtained from regression analysis. Equation (10) is shown graphically in figure 6 for typical values of the constants.

From an analytical view, the arc voltage can be described by Ohm's law:

$$
E_{A}=I_{A} \frac{\ell_{A}}{\sigma_{A} A_{A}},
$$

where $\sigma_{A}$ is an effective arc-column electrical conductivity, $\ell_{A}$ is the arc length, and $A_{A}$ is the effective arc-column cross-sectional area. The value of $\sigma_{A}$ varies as a function of the local arc temperature and composition. Effective is used to accentuate the fact that both the conductivity and cross-sectional area of the arc vary significantly along the length of the arc. Several mechanisms determine the shape of the arc. The electrical conductivity for Ar gas as a function of temperature is shown in figure 7 [15]. The conductivity reaches a plateau at about $15000 \mathrm{~K}$. Experimental investigations have shown that the peak temperature of the arc does not vary significantly over

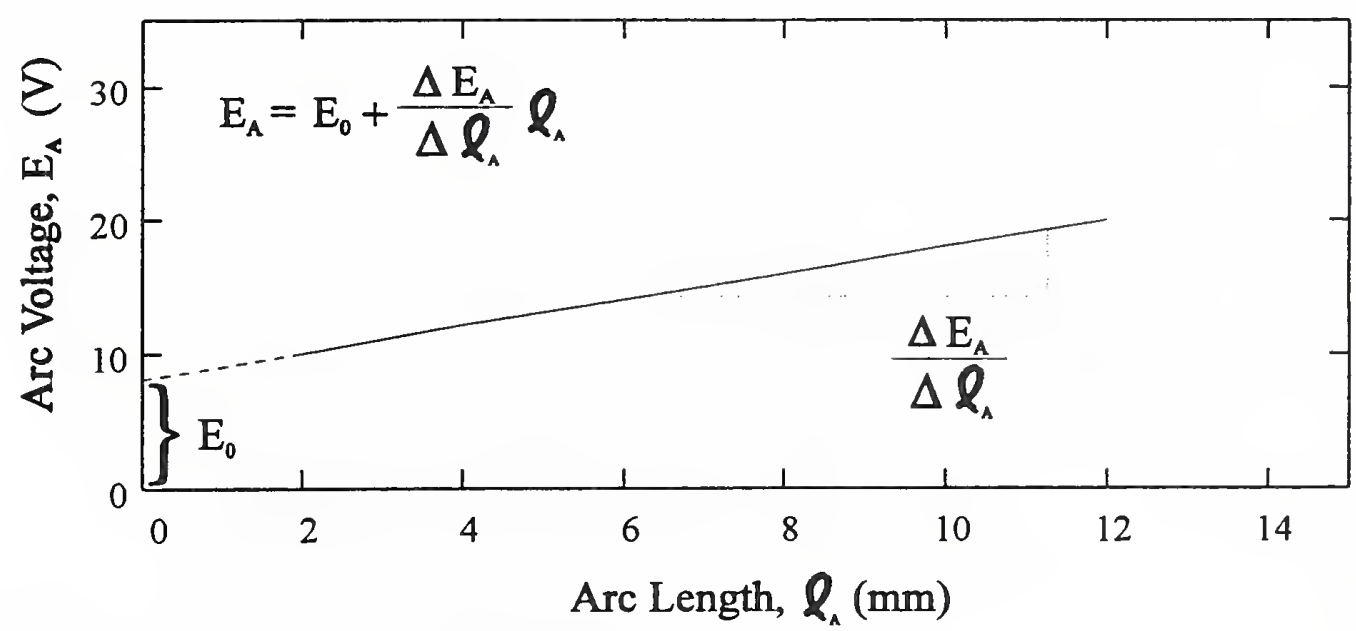

Figure 5. Arc voltage drop as a function of arc length. Arc voltage drop increases linearly with arc length. The voltage drop extrapolated to zero arc length is the sum of the anode and cathode voltage drops. 


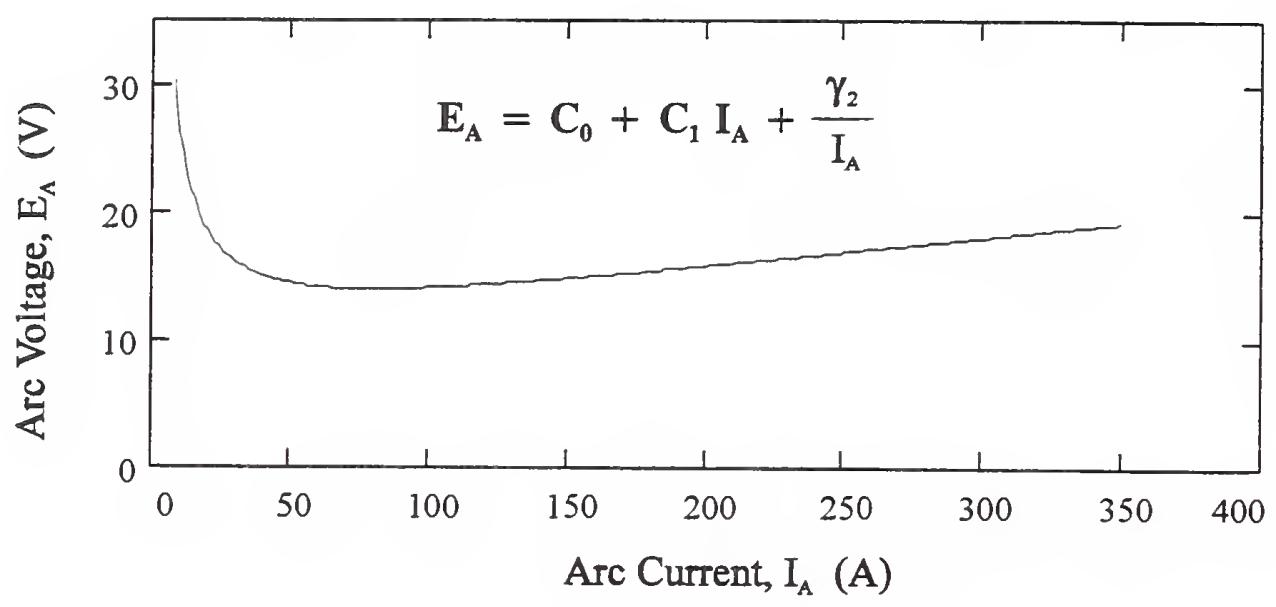

Figure 6. Arc voltage drop as a function of arc current. The arc voltage drop increases with arc current in the range associated with the spraytransfer mode $\left(I_{A}>200 \mathrm{~A}\right)$.

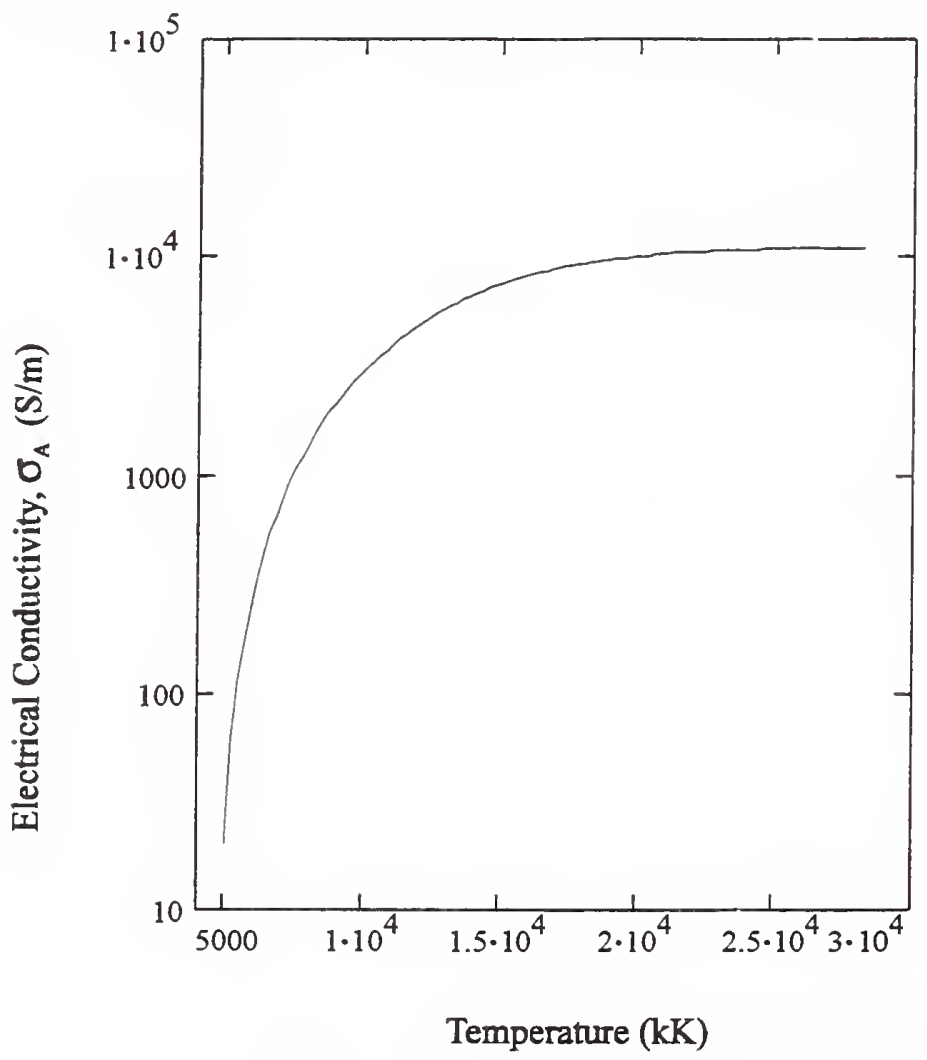

Figure 7. Electrical conductivity of $\mathrm{Ar}$ as a function of temperature. The conductivity reaches a plateau near $15000 \mathrm{~K}$ [15]. 
a large range of current [16]. Furthermore, as suggested in figure 5, the electric field along the arc length is constant, or, rewriting eq (11),

$$
\frac{E_{A}}{\ell_{A}}=I_{A} \frac{1}{\sigma_{A} A_{A}} \approx \text { constant } .
$$

Inspection of eq (12) indicates that for a given current, the product $\sigma_{A} A_{A}$ is a constant. Since the conductivity reaches a plateau, the peak temperature does not vary with current, and the electric field in the arc is constant, the cross-sectional area along the length of the arc changes as required to support the total arc current. The change in arc cross-sectional area can be readily observed [16]. Therefore the arc volume changes in response to changes in arc length and to changes in cross-sectional area through the arc current. These observations, coupled with treatment of the arc as a volume light source (section 3), will be used in section 4 to develop a sensor for arc length and in section 6 to detect droplet detachment.

\subsection{Welding-Process Control}

Arc-welding can be viewed as a process of mass and heat flow [17], which can be visualized with the help of figure 8 . Consider the heat source to be stationary and the joint to be moving to the right. Welding occurs within the thick rectangular box, or process volume, at the center of the figure. The unwelded joint flows into the left side of the volume, heat for melting and filler flow into the top, and heat and material in the form of the completed weld flow out of the right side of the volume. A variety of subprocesses occurs simultaneously within the process volume, such as melting,

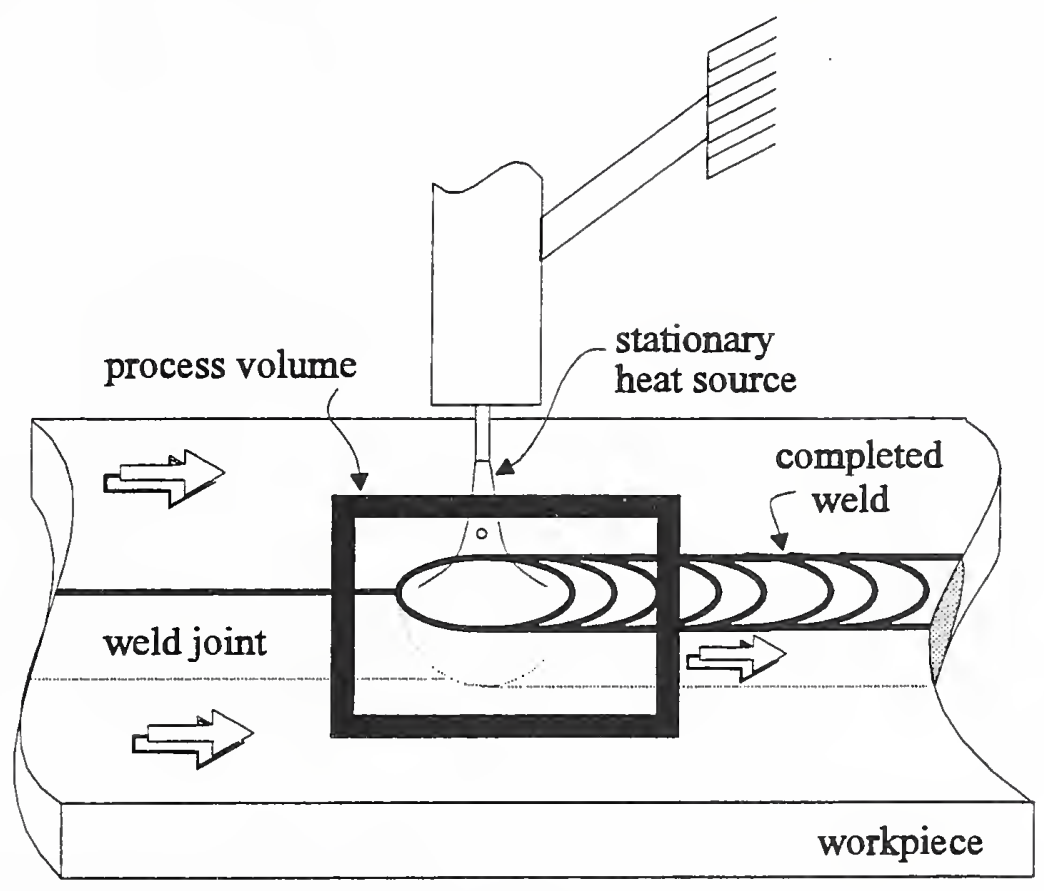

Figure 8. Definition of the process volume. Assuming that the welding torch (heat source) is stationary and the workpiece moves, the unwelded joint flows into the left side of the volume, heat and filler flow in from the top, and the completed weld flows out the right. 


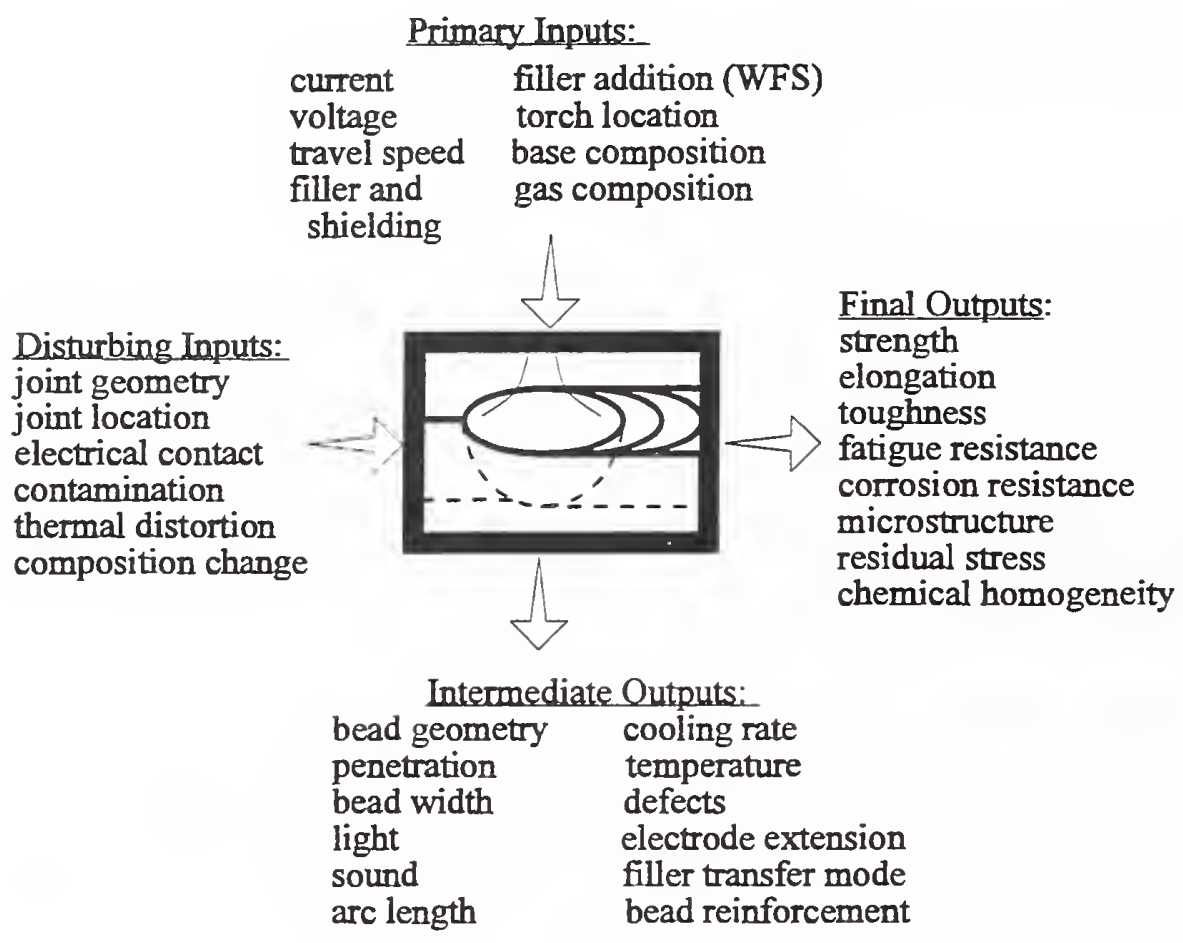

Figure 9. Definitions of welding-process volume input and output. The ultimate goal of welding-process control is to control the final outputs for which there are no in situ sensors. Process control in real time is limited to the primary and disturbing inputs and the intermediate outputs.

gas-liquid-solid reactions, solidification, alloy-solute redistribution, grain growth, phase transformations, diffusion, and the development of residual stresses through thermal expansion and contraction. All contribute to the final properties of the weld.

The ultimate goal of welding-process control is to control the properties of a weld as it emerges from the process volume. Referring to figure 9, the primary properties to be controlled are those of the weld itself, which are considered to be the final process outputs. They include tensile and compressive strength, elongation, toughness, fracture resistance, fatigue strength, and corrosion resistance. These properties inherently depend on the microstructure of the weld metal and the surrounding heataffected zone, which, in turn, depend on the welding-process variables.

\subsubsection{Control Block Diagrams and the Transfer Function}

In control engineering, the use of block diagrams simplifies the representation and design of a control system. Elements of a control block diagram are shown in figure 10 . The elements typically include blocks representing the process, controller, and actuator. Each block consists of an input, output, and transfer function. The transformation of an input to an output is accomplished by a transfer function $K$ defined by 


$$
O / I=K
$$

where $I$ and $O$ designate the input and output of the block. Static and dynamic properties are associated with each transfer function. Under steady-state or equilibrium conditions, the output will eventually assume a fixed value directly corresponding to the fixed input value. Under transient conditions, however, the output may not achieve the value corresponding to the input instantly. Thus, the output may be a function of the input and of time, revealing a dynamic transfer function. Transferfunction dynamics are typically classified by frequency response in terms of the amplitude ratio and phase angle between the output and input [18].

As shown in figure 10 , the blocks are cascaded to produce a functional control system. The desired value of the process or final output is designated as the setpoint and acts as the input to the controller. The controller operates on the setpoint signal to produce a signal that is the input to the actuator. The actuator converts the controller output to a form capable of affecting the process. The process accepts the actuator output as its input and operates on it to produce the desired process output.

An example of the control system depicted in figure 10 is the wire-feed unit used in GMAW. The input to the controller may be a knob on the feed unit representing the WFS setpoint. The transfer function of the controller $K_{C}$ converts the knob position to an electrical signal applied to a power amplifier, the actuator. The amplifier produces electrical power capable of driving a motor through the transfer
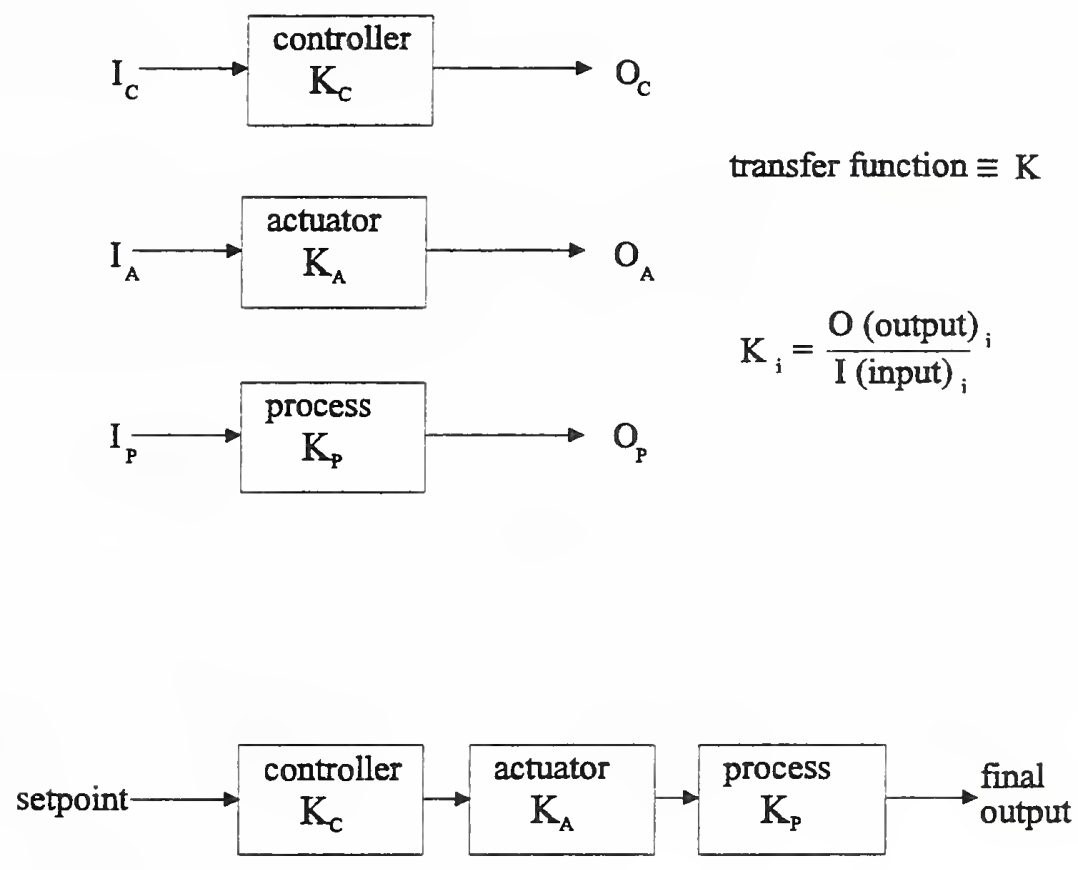

Figure 10. Block diagram representation of a process-control system, including the controller, actuator, and the process. Each component ${ }_{\mathrm{i}}$ has a transfer function, $K_{i}$, defined as the block output, $O_{i}$, divided by the block input, $I_{\mathrm{i}}$. The blocks are cascaded together to form the control system. 
function $K_{A}$. The amplifier is capable of following the input relatively rapidly; therefore, $K_{A}$ is considered static. The transfer function of the process, $K_{P}$, in this case is the conversion of electrical power to mechanical motion of the wire electrode. The electrical power rotates a motor attached to pinch rollers that ultimately drive the wire. Owing to the inertia associated with the motor and rotating mass, the WFS will not respond instantaneously to a change in controller-knob setting; thus, $K_{P}$ is said to be dynamic.

\subsubsection{Open-Loop Control}

The block diagram in figure 11 illustrates the basic form of an open-loop process-control system. In the event that disturbing inputs act on the process, it is impossible for the controller to sense that the process output may have deviated from the desired value. For the above wire-feeder example, the controller would not be capable of detecting slippage of the wire in the pinch rollers. (Slippage is a disturbing input.) To maintain the process output at the desired value, regardless of the effects of disturbing inputs, the controller must have access to information about the actual state of the process; in other words, the process-control loop must be closed.

\subsubsection{Closed-Loop Control}

There are two primary closed-loop methods to control a process: feedback control and feedforward control. In both methods, a sensor is required to provide the controller with information about the process. Like the controller and the process, the sensor has a transfer function associated with it. The sensor must convert some measure of process information to a form that is compatible with the controller setpoint.

In feedforward control (see fig. 12), disturbing inputs are sensed as they reach the process. On the basis of sensory information, the controller adjusts the process input such that the impending disturbances have minimal impact on the process output. A distinct advantage of feedforward control is that the disturbances are accounted for before the process goes out of control. A limitation of feedforward control is the

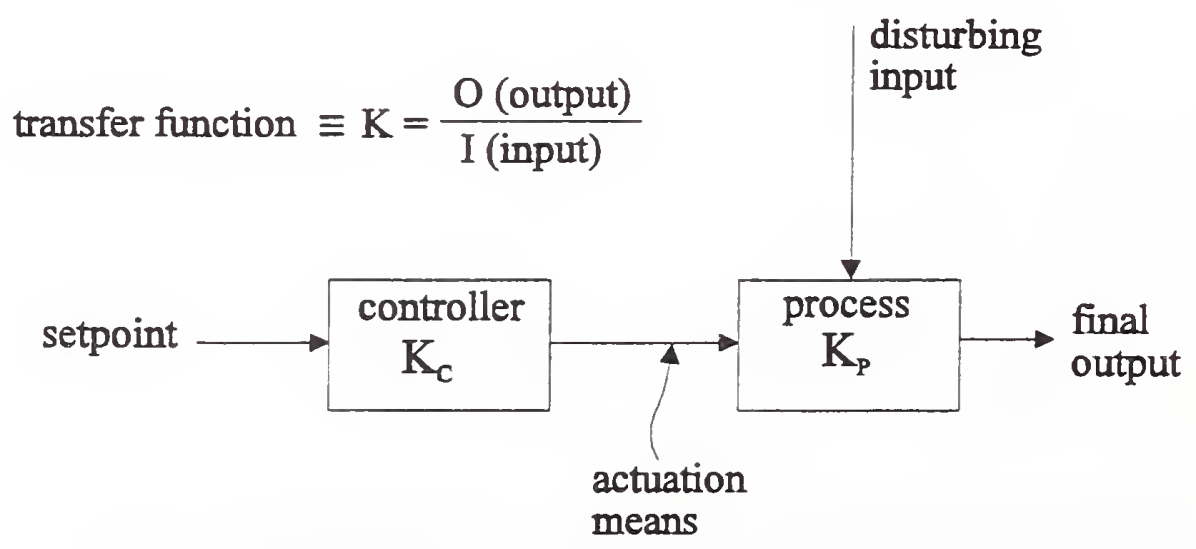

Figure 11. Block diagram of open-loop process control. The open-loop control cannot correct for final output deviations caused by the disturbing inputs. 


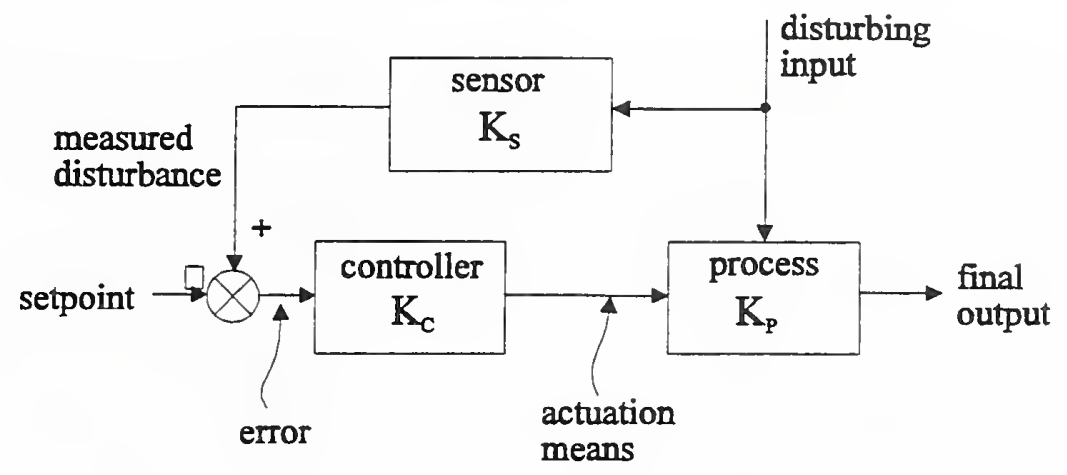

Figure 12. Block diagram of closed-loop, feedforward control. A sensor detects disturbing inputs about to influence the process. That information is fed to the controller to make adjustments to the process input that will minimize the effects on the process output.

requirement that sensors detect disturbances prior to their influence on the process. In many cases during welding, it is not possible to be sensitive to all conditions that may disturb the welding process.

In feedback control, a process output is sensed and compared with the setpoint. The difference between the measured output value and setpoint value (error) is used by the controller to actuate a change in the process to drive the error to 0 . Figure 13 shows a block diagram for a system based on feedback of the final outputs. In some cases, it is impossible to sense the final output directly, but it is possible to sense an intermediate output that is related to the final output. Figure 14 shows a block diagram for a feedback system based on intermediate-output feedback. An advantage of feedback control is that only process output must be sensed, eliminating the need to detect a wide variety of disturbing inputs. A disadvantage of feedback control is that the process must actually deviate from the setpoint before any corrective action is taken.

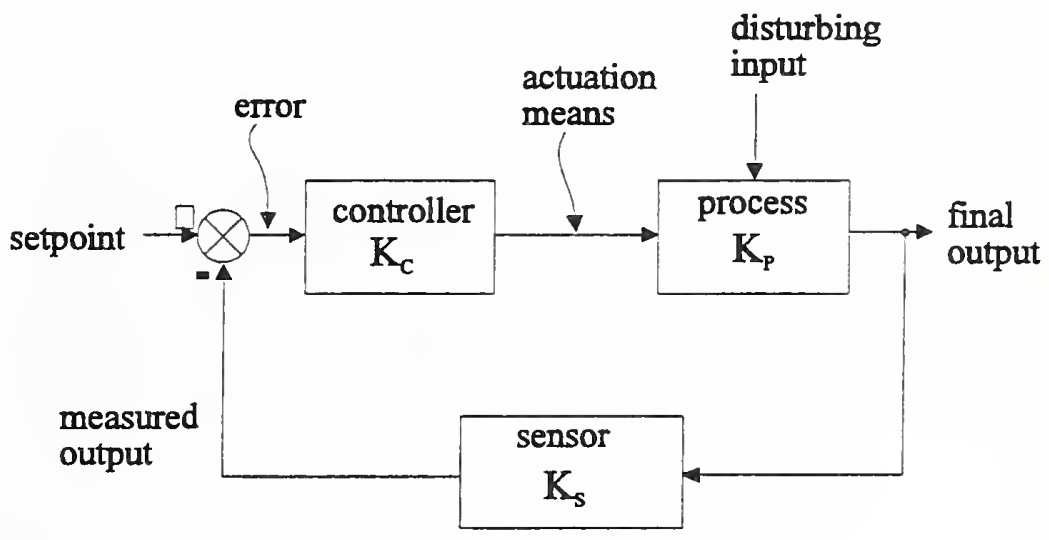

Figure 13. Block diagram of closed-loop, feedback control based on sensing the state of the final output, which operates the controller. 




Figure 14. Block diagram of closed-loop, feedback control based on intermediate-output sensing. A relationship between the intermediate output and the final output enables indirect control of the final output.

\subsubsection{Welding-Process Controller}

To design and implement a controller, the process and actuator transfer functions must be defined, and an appropriate sensor must be available. For processes that can be accurately modeled and linearized, a simple, linear controller can be designed and applied with a suitable sensor $[18,19]$. Welding rarely fits into this category of processes since most welding processes are complex, nonlinear, and difficult to model. An approach that has made significant advances when applied to designing welding controllers includes the use of artificial intelligence [20,21]. By employing artificial intelligence, we can design a controller based only on the observed input/output states of a process without a complete understanding of the relationship between them.

As previously indicated, the goal of welding-process control is to control the properties of the weld (see fig. 9). Available sensors cannot directly measure the final output properties in situ. At this point, it can be argued that it is not possible to have true feedback control of the welding processes. As a result, welding-process control is limited to indirect control of weld properties by controlling the parameters that can be readily sensed: the primary and disturbing inputs and the intermediate outputs. In most welding applications, the shielding-gas, electrode, and base-metal compositions and the joint design are fixed before welding, but they are subject to natural variations. Of the remaining parameters, WFS and current are the easiest to actuate and thereby to influence the process. In this study, simple controllers were designed and applied to demonstrate the real-time control of arc length and droplet frequency.

\subsection{Summary}

To date, it is not possible to achieve true closed-loop control of all of the weld properties as they emerge from the process volume owing to the lack of appropriate sensors. The approach taken, then, is to control the weld properties indirectly by gaining better control of the process inputs. 
The existing knowledge and models of the GMAW process show that arc length and droplet-transfer mode are important parameters to control; thus, they provide a basis for designing such controllers. Of the process input parameters, WFS and current are the most easily changed during welding and have well-defined relationships with both arc length and transfer mode. Either WFS or current could be actuated by a controller to control arc length and transfer mode, given a suitable sensor. Although arc length is proportional to arc voltage drop, the composite nature of the process voltage inhibits the estimation of arc length. The detection of droplet detachment from either the current or process-voltage signals is impaired by the small droplets associated with the spray-transfer mode. The concept of arc-light sensing, described in section 3, offers an additional sensory input upon which arc-length and transfer-mode controllers can be designed.

\section{Emission and Sensing of Arc Light}

In the following discussion of the emission and sensing of arc light for the arcwelding control, the term light refers to all wavelengths of electromagnetic radiation emitted by the arc.

\subsection{Electrical Conduction in the Welding Arc}

The welding arc is an electrically conductive gas called plasma, which is considered to be the fourth and most complex state of matter. The arc-column plasma is produced by the electrical breakdown of the normally nonconducting gases between the electrode and the workpiece. Electrical breakdown of the gas occurs through ionization, the act of stripping electrons from atoms; it is detailed elsewhere [22,23]. The ionization process produces free, charged particles: ions and electrons. Electrical conduction takes place through the motion of the free, charged particles constituting the plasma. It is instructive to consider conduction in a solid metal first.

Conduction in a metal can be viewed by imagining the metal to be a lattice of positive ions through which a sea of electrons is free to move under an applied electric field [24]. If a potential difference is set up between the ends of a solid metal, electrical current flow occurs as the electrons move from the negative towards the positive end while the positive ions remain fixed in position. Some of the energy that the electrons acquire from the electric field is transferred to the ions during collisions and is converted into heat, the origin of ohmic heating.

In a plasma, both electrons and ions are present. However, unlike those in a metal, the ions are not fixed in position by a lattice structure and are free to move under the influence of the electric potential between the electrode and the workpiece. Thus, conduction in a plasma takes the form of two currents (electron and ion currents) flowing in opposite directions. Just as in a metal, the collision of electrons, ions, molecules, and atoms generates ohmic heating within the plasma. The heating produces the high temperatures associated with the plasma volume, supports the ionization process, and hence, stabilizes the arc into a self-sustaining, continuous discharge. 


\subsection{Arc-Energy Balance}

From the principle of conservation of energy, the power produced in the arc must equal the power lost by it under steady-state conditions. A model for the electrical power developed in the $\operatorname{arc} P_{A}$ can be obtained by multiplying eq (11) by the arc current, $I_{A}$, yielding

$$
P_{A}=I_{A}^{2} \frac{\ell_{A}}{\sigma_{A} A_{A}} .
$$

A model of the power lost by the arc is easy to conceptualize by including terms for the energy-transport processes known to be in operation [25]:

$$
P_{A}=P_{K}+P_{V}+P_{L}
$$

The terms in eq (15) account for power loss due to conduction $P_{K}$, convection $P_{V}$, and radiation $P_{L}$. Using simplifying assumptions about the shape of the arc and mass flow within the arc, analytical models for conductive and convective losses have been developed [26-28]. A model of the radiation-loss term is difficult to develop analytically because it is a strong function of arc composition and temperature [29]. A simple model of arc radiation has recently been developed wherein the arc is considered a homogenous volume without metallic vapor [30]. However, in nearly all arc models, the radiation-loss term is incorporated by using the experimental results of Evans and Tankin [31]. The complexity of arc radiation can be appreciated by reviewing the origin of arc-light emission.

\subsection{Origin of Arc-Light Emission}

Electromagnetic radiation originates from particles within the emitting source and can be characterized by its wavelength spectrum. Figure 15 provides a broad overview of common radiation nomenclature and production mechanisms as a function of wavelength. The light-emission pattern or spectrum of the arc plasma is typically displayed in terms of light intensity versus wavelength. The visible-emission spectrum of a GMAW arc while welding steel with $98 \% \mathrm{Ar}+2 \% \mathrm{O}_{2}$ shielding gas is shown in figure 16 [33]. It consists of high-intensity lines at specific wavelengths and a lower intensity continuum between the lines.

The emission of arc light originates from the particles within the arc plasma: free atoms, ions, and electrons. Their various electronic energy levels are diagrammed in figure 17. The zero-energy level $U_{O}$ is the ground state (lowest energy-bound state), with the higher bound states $U_{i}$ at higher, positive energy levels. The energy $U_{I}$ is the ionization potential, that is, the energy required to produce ionization from the ground state. Energies above $U_{I}$ denote that ionization has taken place and free electrons exist. The basic unit of radiative energy, the photon, provides a convenient method for discussing the radiative processes.

Radiative processes include photon emission and photon absorption. When a photon is emitted or absorbed, the energy of the emitting or absorbing particle is correspondingly decreased or increased. Figure 17 shows the three types of energy emission or absorption transitions that can occur: bound-bound (BBE, BBA), 


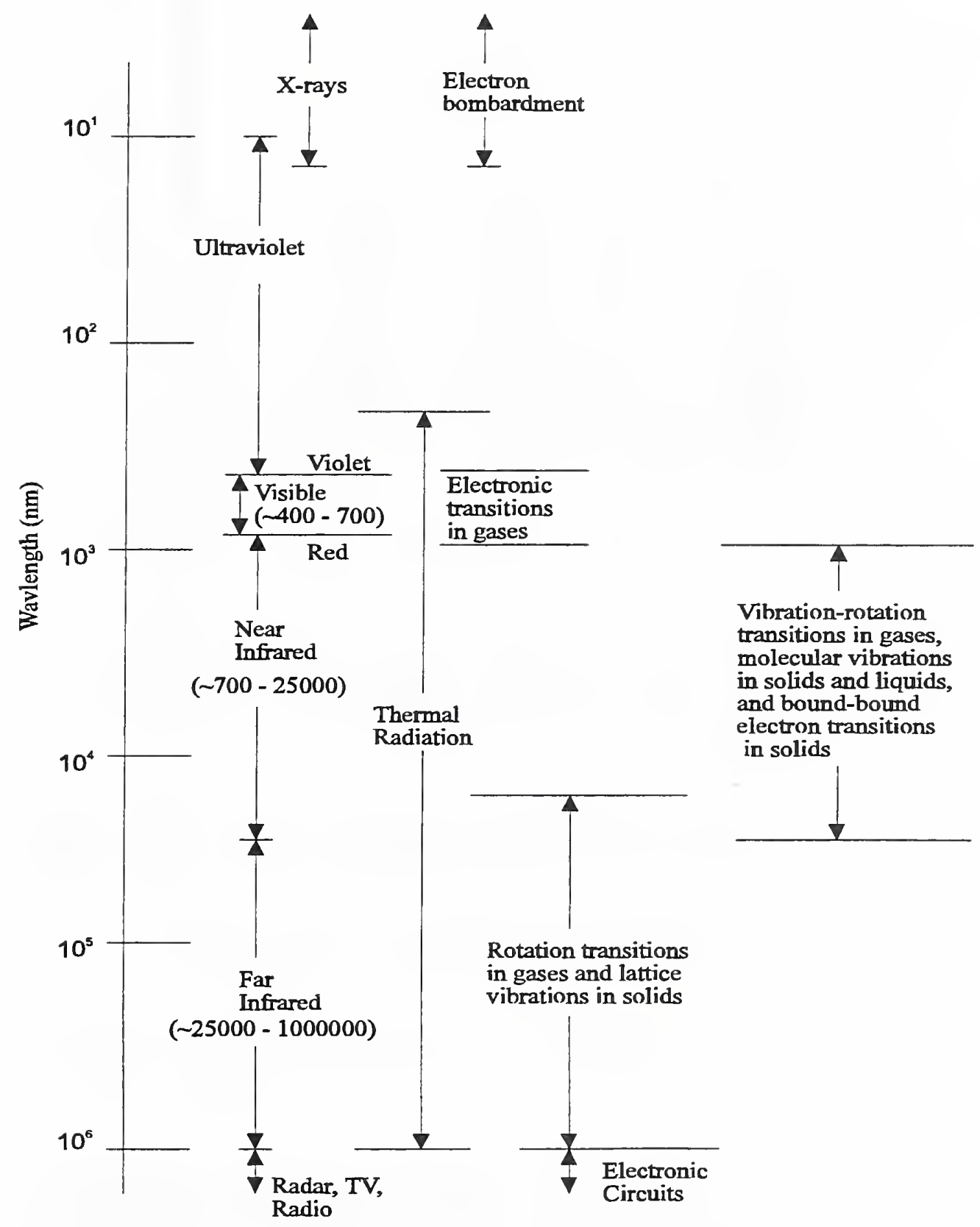

Figure 15. Overview of nomenclature for electromagnetic radiation and production mechanisms as a function of radiation wavelength. The electronic transitions of the particles in the welding arc emit light in the visible and surrounding region [32]. 


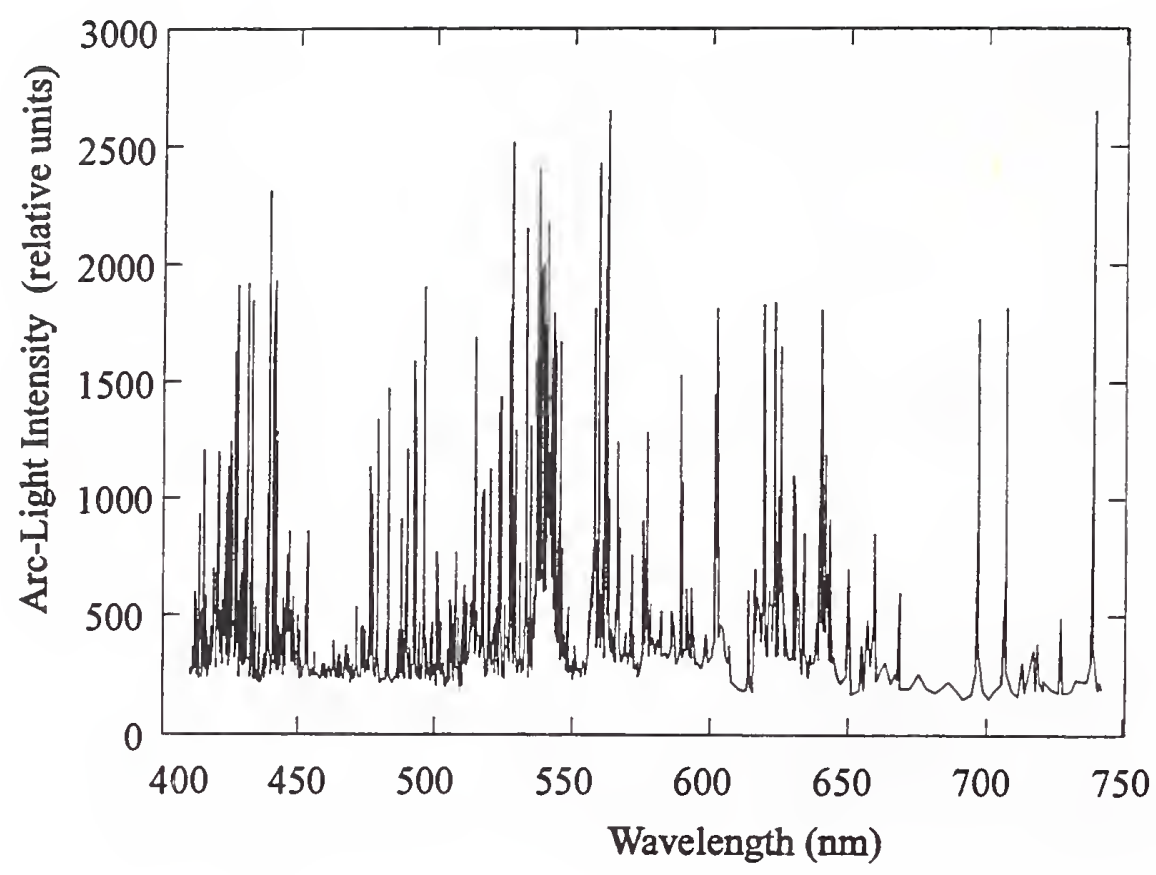

Figure 16. The visible emission spectrum of an arc during the welding of steel with $98 \% \mathrm{Ar}+2 \% \mathrm{O}_{2}$ shielding gas using the GMAW process [33].

bound-free (BFA, FBE), and free-free (FFE, FFA). The magnitude of the energy change is related to the wavelength of the emitted or absorbed radiation. The energy of a photon is

$$
\Delta U=\hbar \nu=\hbar \frac{c}{\lambda},
$$

where $\hbar$ is Planck's constant, $\nu$ is the frequency of the photon, $c$ is the speed of light, and $\lambda$ is the wavelength of the photon.

\subsubsection{Bound-Bound Transitions}

In a transition between two bound states, the quantum nature of the process dictates that the absorbing particle can go to only one of the discrete higher energy levels (process BBA in fig. 17). Consequently, for the photon to be absorbed, its wavelength must be a discrete value. For example, a particle in the ground state in figure 17 can absorb photons with wavelengths $\hbar c /\left(U_{2}-U_{1}\right), \hbar c /\left(U_{3}-U_{2}\right)$, or $\hbar c /\left(U_{4}-U_{1}\right)$ and undergo a transition to a higher bound-energy level. Photons with wavelengths in the range $\infty>\lambda>\hbar c / U_{1}$ cannot be absorbed. For an energy transition from an excited bound state $U_{3}$ down to bound state $U_{2}$, a photon is emitted with energy $U_{3}-U_{2}=\hbar c / \lambda$ (process BBE in fig. 17). The wavelength of the emitted energy is then $\lambda=\hbar c /\left(U_{3}-U_{2}\right)$, so a fixed wavelength is associated with the transition from one specific energy level to another. Thus, the emitted radiation will be seen as a spectral line at the corresponding wavelength. 


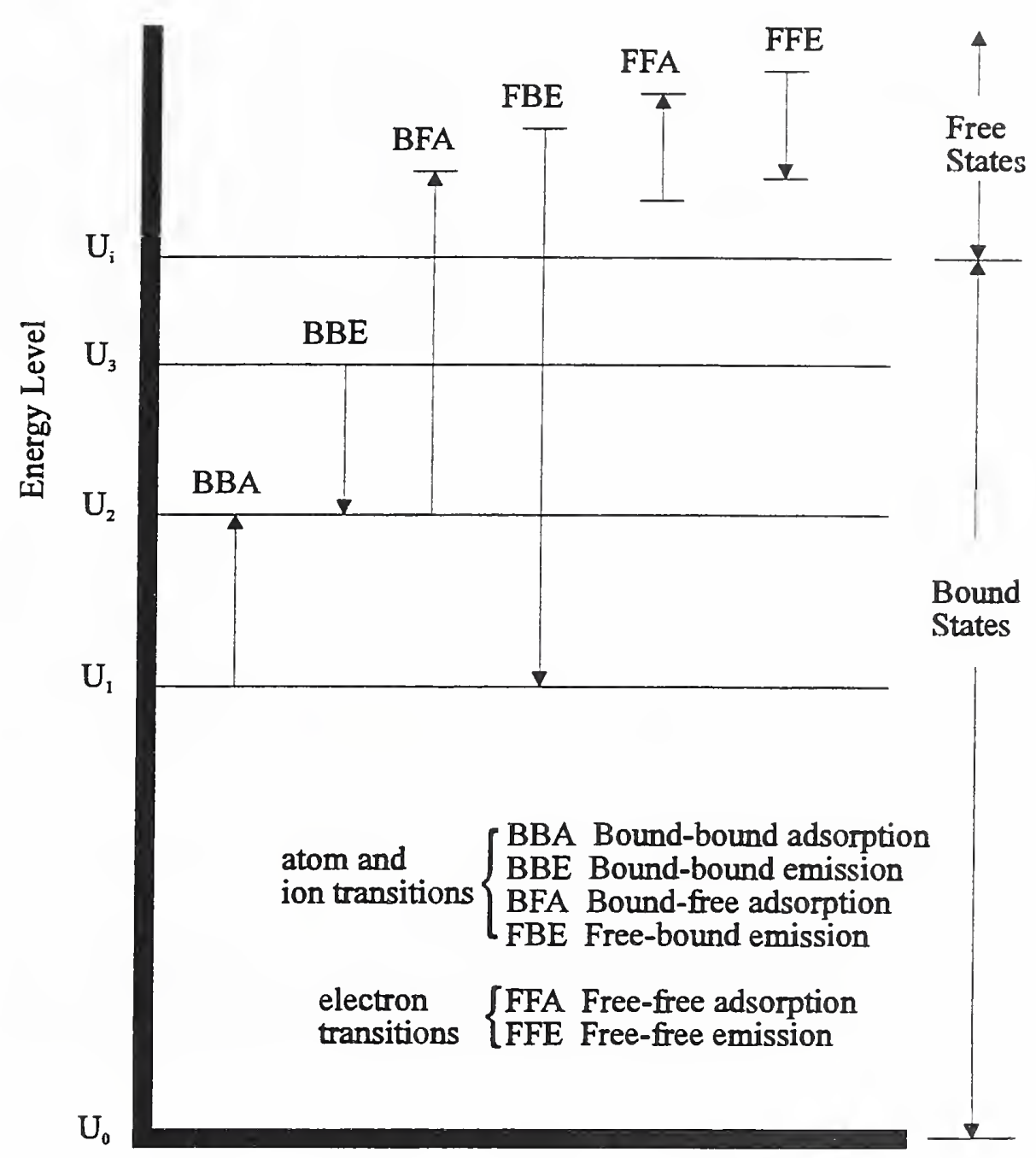

Figure 17. Energy-level diagram showing levels and transitions for an atom, ion, and electron. $U_{0}$ is the ground state and $U_{1}, U_{2}, \ldots$ are higher, positive energy levels of atoms and ions. $U_{I}$ is the ionization energy.

The BBE processes are responsible for the appearance of the spectral lines in figure 17. The intensity of each line is determined by the number of atoms undergoing the BBE process at each wavelength. The BBE transitions of excited atoms in the arc plasma occur at wavelengths in the visible region $(400-700 \mathrm{~nm})$ and in the nearultraviolet and near-infrared regions (see fig. 15).

The absorption of certain radiation wavelengths gives rise to the terms optically thick and optically thin that are used to describe the opacity of the plasma (its ability to absorb radiation) [34]. The arc is optically thick to radiation wavelengths that are absorbed and optically thin to wavelengths not absorbed. As discussed in section 3.4, a good approximation is to consider the arc to be optically thin, indicating that most of the radiation generated within the arc volume readily escapes. 


\subsubsection{Bound-Free Transitions}

Process BFA in figure 17 is a bound-free-absorption or photoionization process: An atom absorbs a photon with sufficient energy to cause ionization. The resulting ion and electron are free to take on any kinetic energy; hence, the BFA process is a continuous function of wavelength as long as the photon energy $\hbar c / \lambda$ is sufficiently large to cause ionization. The reverse, FBE, is free-bound emission or photorecombination: An ion and free electron combine, a photon is released, and the energy of the resulting atom drops to that of a discrete bound state. The FBE process produces a continuous-emission spectrum, as the combining particles can have any initial kinetic energy. The FBE process is, in part, responsible for the background continuum in figure 16 .

\subsubsection{Free-Free Transitions}

In the arc plasma, a free electron can pass near an ion, interact with its electric field, and produce a free-free transition. The electron can absorb a photon, thereby going to a higher kinetic energy level (process FFA in fig. 17). The electron can emit a photon as a result of the interaction and drop to a lower free-energy level (process FFE). The FFE process is often termed bremsstrahlung, meaning brake radiation. Since the initial and final free energies of the electron can have any values, a continuous emission spectrum is produced that contributes to the background continuum in figure 16.

The foregoing discussion shows that analytical modeling of the radiation loss term in eq (15) is a formidable task. The arc plasma is composed of a variety of particles originating from the shielding gas, the electrode, the weld pool, and the atmosphere. Each particle type has a number of energy levels, and each may encounter other particles, leading to a large number of possible combinations or energy transitions. Steep thermal gradients in the arc cause large variations in particle density, further complicating the situation.

\subsection{Experimental Observations of Arc Radiation}

Welding-arc radiation has been the topic of numerous experimental works [29,35-37]. Spectrographic studies have been used primarily for estimating temperature and concentration of components in the arc plasma. The study of how arc radiation is related to arc geometry and welding parameters, a topic of prime interest here, has been limited to gas-tungsten-arc welding (GTAW) [38-40], and it does not include the complication of metal transfer across the arc.

As previously indicated, the experimental results of Evans and Tankin [31] are used extensively in arc models to account for radiative losses. Their work used GTAW with Ar shielding gas and constant current and arc length (250 A and $6 \mathrm{~mm}$ ). Figure 18 shows the total radiation in the wavelength range $200 \mathrm{~nm}$ to $3500 \mathrm{~nm}$ as a function of temperature. For this range of wavelengths, radiative power is a highly nonlinear function of temperature. Evans and Tankin's results also showed that the arc can be assumed to be optically thin (thus, internal absorption can be neglected). Kim investigated light emissions from GTAW with a variety of base-metal compositions using Ar and He shielding gases [37]. With the arc length for all welds fixed at $8 \mathrm{~mm}$, he found that the intensities of the FeI line (due to emission from neutral 


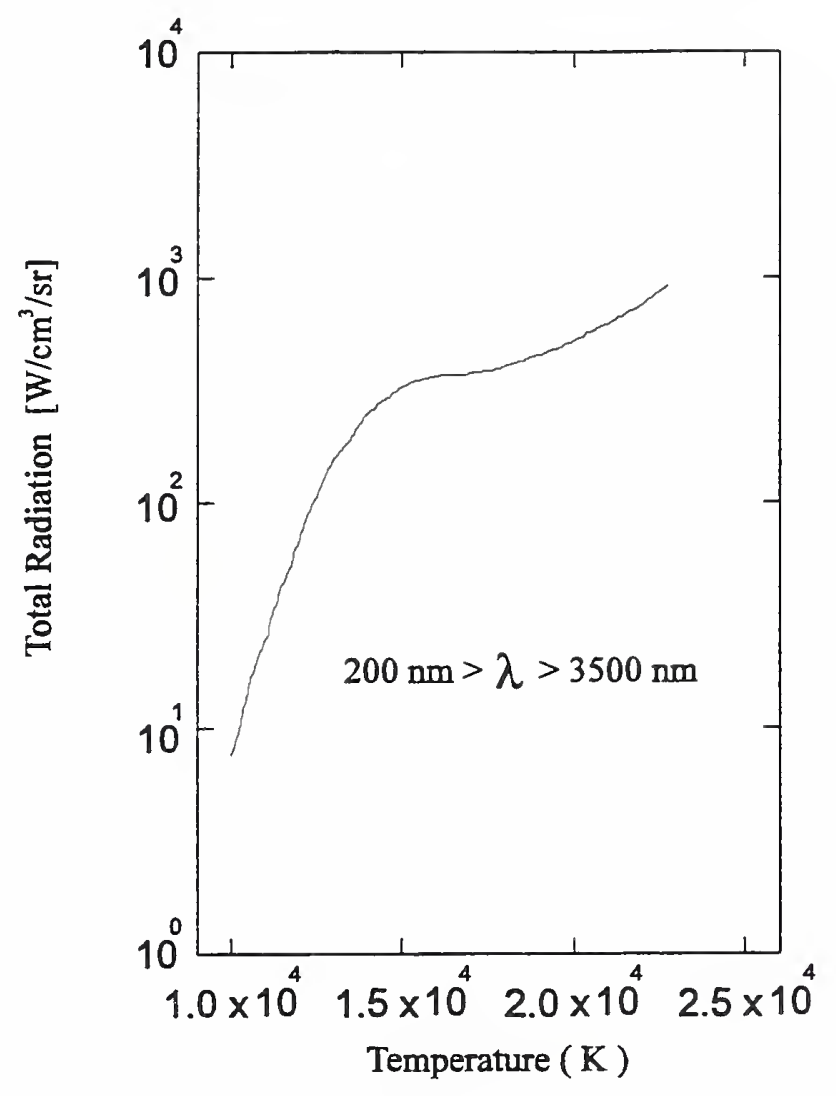

Figure 18. Arc radiant power in the wavelength range $200 \mathrm{~nm}$ to $3500 \mathrm{~nm}$ as a function of temperature for a 200-A, 6-mm GTAW arc in Ar. The welding arc can be reasonably approximated as being optically thin [31].

atoms) and the FeIl line (due to emission from singly ionized ions) increased linearly with current while welding AISI-304 stainless steel with Ar shielding gas (see fig. 19).

In a study of arc efficiency in GTAW, Ghent et al. [38] measured the power radiated from the arc as a function of arc current and length. Arc radiant power was obtained indirectly by measuring the heat collected by a water-cooled coil surrounding the arc (see fig. 20). They determined that the increase in arc radiation was approximately linear with both arc length and current (see fig. 21).

Alum observed similar results, also for GTAW [39]. He concluded that the power lost from the arc column by radiation must be proportional to the product of the arc voltage and current and provided an empirical expression,

$$
P_{L}=E_{A} I_{A} \beta
$$

where $\beta$ is the fraction of arc power lost by radiation and $P_{L}$ is the arc radiative power. Typical values for $\beta$ ranged from 18 to 25 percent. 


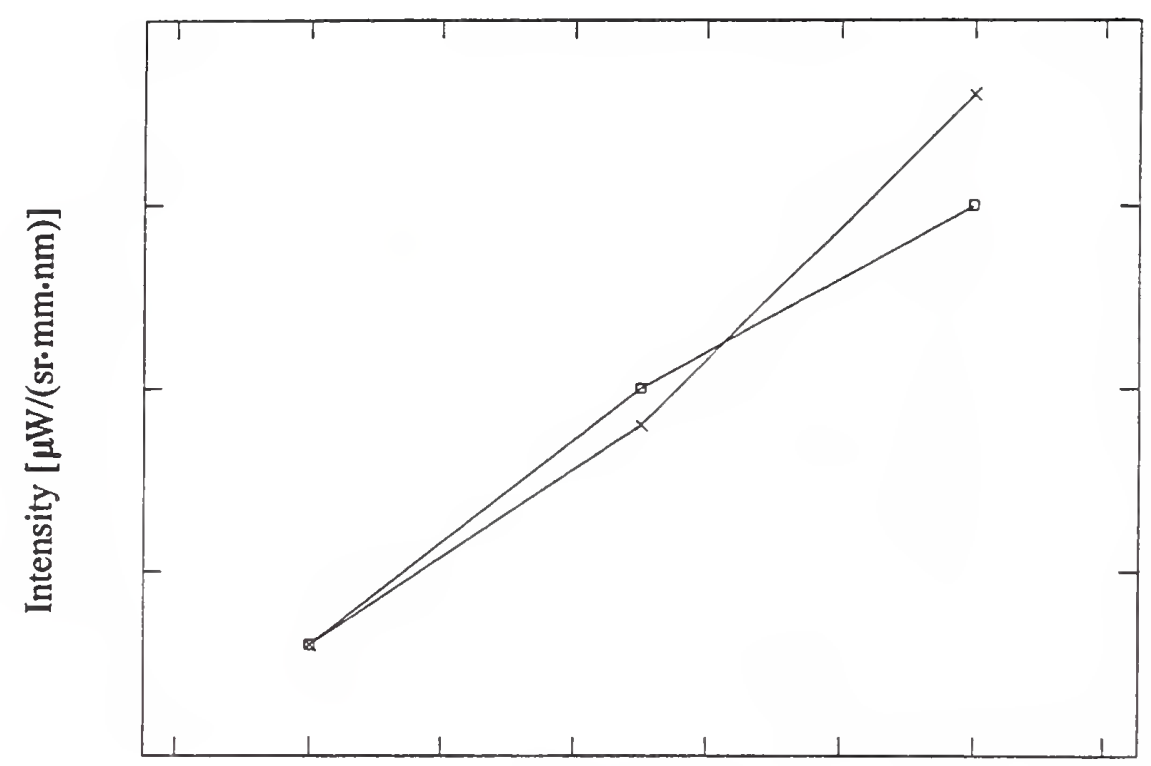

Current (A)

- FeI

$\rightarrow$ FeII

Figure 19. The change in FeI- and Fell-line intensities with current during GTAW (Kim [37]). Welding was conducted on AISI-304 stainless steel using Ar shielding gas.

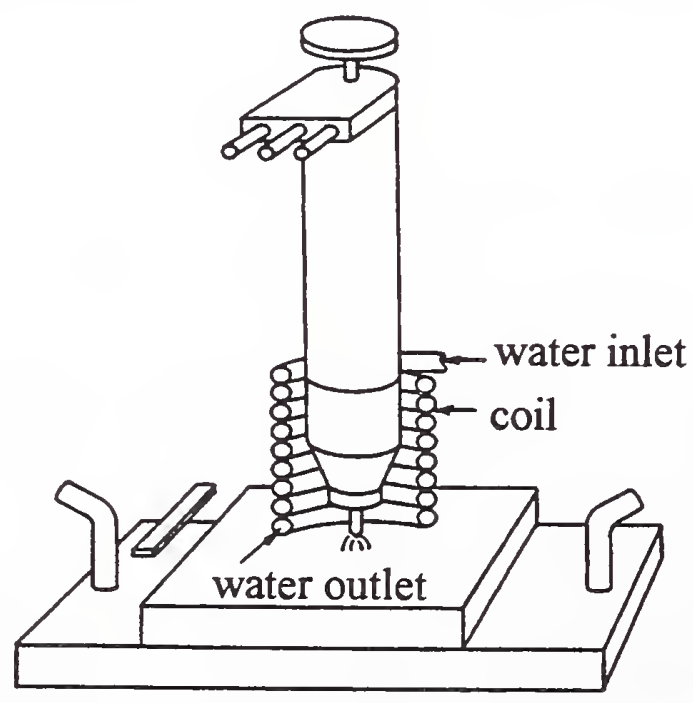

Figure 20. The water-cooled coil used by Ghent et al. [38] to collect arc radiation as a function of arc length and current. 


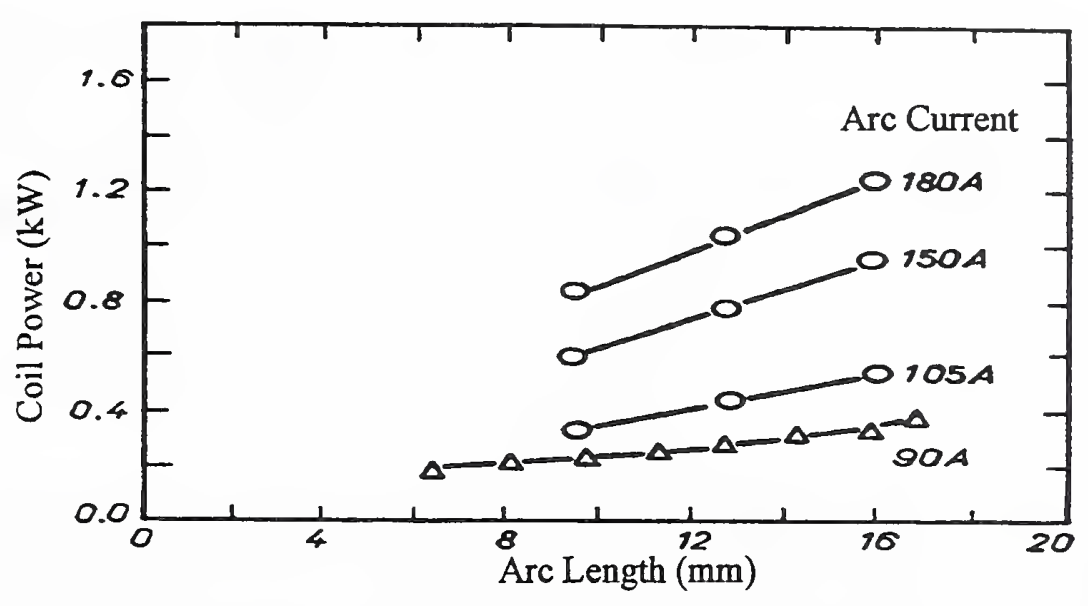

Figure 21. Arc radiant power collected in the water-cooled coil as a function of arc length and current. Power in the coil increases approximately linearly with both current and length [38].

\subsection{Arc-Light Sensing}

Two broad classes of radiation detectors are available: thermal and photon [40]. Both are used to convert incident radiant power input to a proportional-voltage-signal output. Thermal detectors first convert incident-photon flux into heat through absorption by a thermal-mass element. The temperature rise of the thermal mass is then measured and amplified to provide the output signal. Photon detectors convert encounters between photons and the detector, such as the separation of an electronhole pair in a photodiode, into an electronic event. The result is a change in the electronic characteristic of the detector that is amplified to provide the output signal. The frequency response of most thermal detectors is limited by the heat-dissipation time constant of the thermal mass. Since the time constant of a photon detector is limited by an electronic process, the frequency response of a photon detector is significantly greater than that of a thermal detector. A commercially available photodiode (a type of photon detector) was used during this study.

To calculate the radiant intensity (power per unit steradian) incident on a detector, it is necessary to consider the source/detector geometry. It is instructive to consider a planar source first. Assuming that the source is not collimated, the detector intercepts only a small fraction of the total power radiated from the source. Using figure 22 as a reference, the incremental intensity $d \Phi$ incident on a section of a planar detector $d A_{D}$ from a portion of a planar source $d A_{S}$ is given by

$$
d \Phi=\frac{L_{S} \cos \theta_{S} \cos \theta_{D}}{R_{D}^{2}} d A_{S} d A_{D},
$$

where $L_{S}$ is the radiance, $\theta_{S}$ and $\theta_{D}$ are the angles that the source and detector make with the line-of-sight axis, and $R_{D}$ is the distance between the source and detector $[32,41]$. 


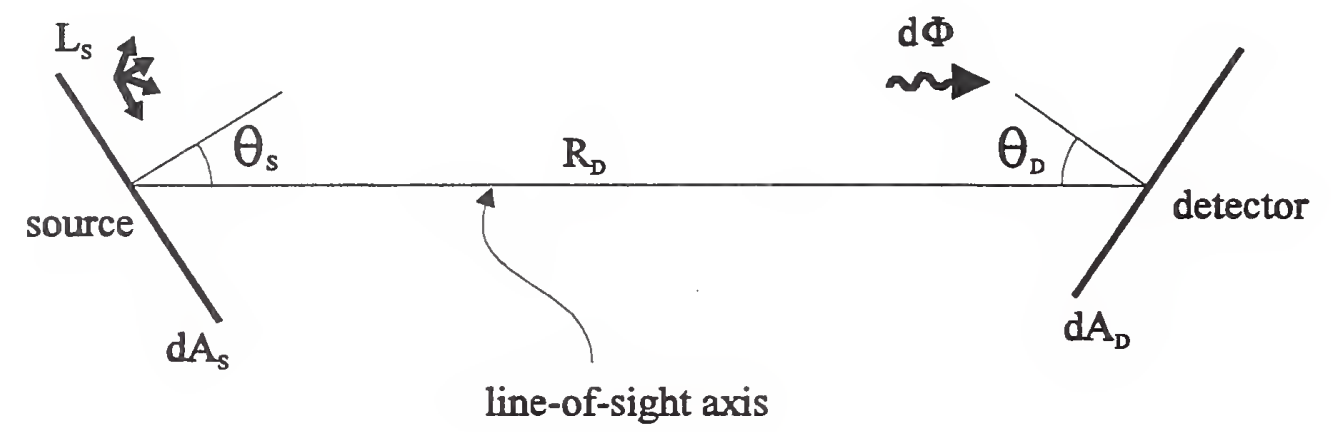

Figure 22. Source/detector configuration. $L_{S}$ is the radiance of the source with area $d A_{S} ; d \Phi$ is the incremental intensity incident on a section of a planar detector with area $d A_{D} ; \theta_{S}$ and $\theta_{D}$ are the angles that the source and detector, respectively, make with the line-of-sight axis; and $R_{D}$ is the distance between the source and detector.

Integrating eq (18) over the total source and detector areas gives the radiant intensity on the detector:

$$
\Phi=\frac{L_{S} A_{S} \cos \theta_{S} A_{D} \cos \theta_{D}}{R_{D}^{2}} .
$$

Several assumptions can be used to convert eq (19) for use with the welding arc as the source. Assuming the arc is optically thin, the arc becomes a volume source, and a term $L_{A}$ can represent the total power per unit volume per steradian emitted by the arc. The volume of the arc is defined by $V_{A}$. Furthermore, the arc is assumed to radiate isotropically in all directions; thus the term $\cos \theta_{S}$ in eq (19) is 1 . The sourcedetector relationship for the welding arc can then be written as

$$
\Phi=\frac{L_{A} V_{A} A_{D} \cos \theta_{D}}{R_{D}^{2}} .
$$

The detector converts the incident radiant intensity to a proportional output voltage $E_{D}$,

$$
E_{D}=\alpha_{1} \Phi=\alpha_{1} \frac{L_{A} V_{A} A_{D} \cos \theta_{D}}{R_{D}^{2}}
$$

where $\alpha_{1}$ is a proportionality constant to account for the spectral response of the detector. In practice, $A_{D}$ and $R_{D}$ are constants, so a ratio involving the two is also a constant:

$$
\alpha_{2}=\frac{A_{D}}{R_{D}^{2}}
$$


The radiant intensity emitted by the arc can be defined as $P_{L}$ where

$$
P_{L}=L_{A} V_{A}
$$

By substituting eqs (22) and (23) into eq (21), the detector voltage can now be written

$$
E_{D}=\alpha_{1} \alpha_{2} P_{L} \cos \theta_{D}
$$

Finally, consolidating the constants $\alpha_{1}$ and $\alpha_{2}$ gives

$$
E_{D}=\alpha P_{L} \cos \theta_{D}
$$

Thus, the detector voltage is proportional to the power lost by the arc in the form of radiation and to the cosine of the angle the detector makes with the line-of-sight axis. The proportionality term $\alpha$ can also be used to account for modifying the spectral response of the detector by placing optical band-pass filters in front of the detector.

\subsection{Summary}

Electrical conduction is accomplished through the motion of the ions and electrons in the arc plasma. The collision of particles, which generates the heat in the arc volume required to sustain the arc, is also responsible for the emission of arc light. Although it is difficult to obtain an analytical model of arc radiation, experimental observations have shown that arc-light intensity increases with both arc length and arc current.

An arc-light detector provides a relative measure of the power lost by the arc in the form of radiation. The output signal of the detector is dependent on its spectral response and on its orientation and location relative to the arc source. The spectral response of the detector can be modified by placing optical band-pass filters in front of the detector. The concepts outlined in this section provided the basis for the subsequent development of arc-length and droplet-detachment sensors, which are described in the following sections.

\section{Experimental Apparatus}

\subsection{Welding Equipment}

The welding equipment consisted of a conventional, constant-voltage power source [silicon-controlled rectifier (SCR)], a transistorized, series-linear current regulator, a capstan wire feeder, and a straight-body machine torch, as depicted in figure 23 . The torch was held stationary while a motorized travel carriage moved the workpiece beneath it. Both the current regulator and wire feeder accepted analog signals from 12-bit digital-to-analog converters (DACs) within the data-acquisition computer to control welding current $(0-1000 \mathrm{~A})$ and WFS $(0-212 \mathrm{~mm} / \mathrm{s})$. Welding could also be accomplished without the use of the current regulator by manually setting the output voltage on the power source. 
The current regulator was used to reduce the normal SCR power-source ripple and to provide a constant current. The frequency-response curves of the current regulator are shown in figure 24; it was capable of faithfully reproducing the input signal with small phase angles up to $500 \mathrm{~Hz}$. As shown in figure 25, the wire feeder was able to track the input signal up to $7 \mathrm{~Hz}$.

\subsection{Instrumentation}

A Hall-effect transducer located in the welding circuit was used to measure arc current (0-600 A) with an absolute error of 1 percent (see fig. 23). The process voltage $(0-50 \mathrm{~V})$, sensed across the power source output, was measured with an absolute error of 0.5 percent. Wire-feed speed $(0-325 \mathrm{~mm} / \mathrm{s})$ was measured within $2 \mathrm{~mm} / \mathrm{s}$ by a transducer mounted where the electrode entered the wire feeder.

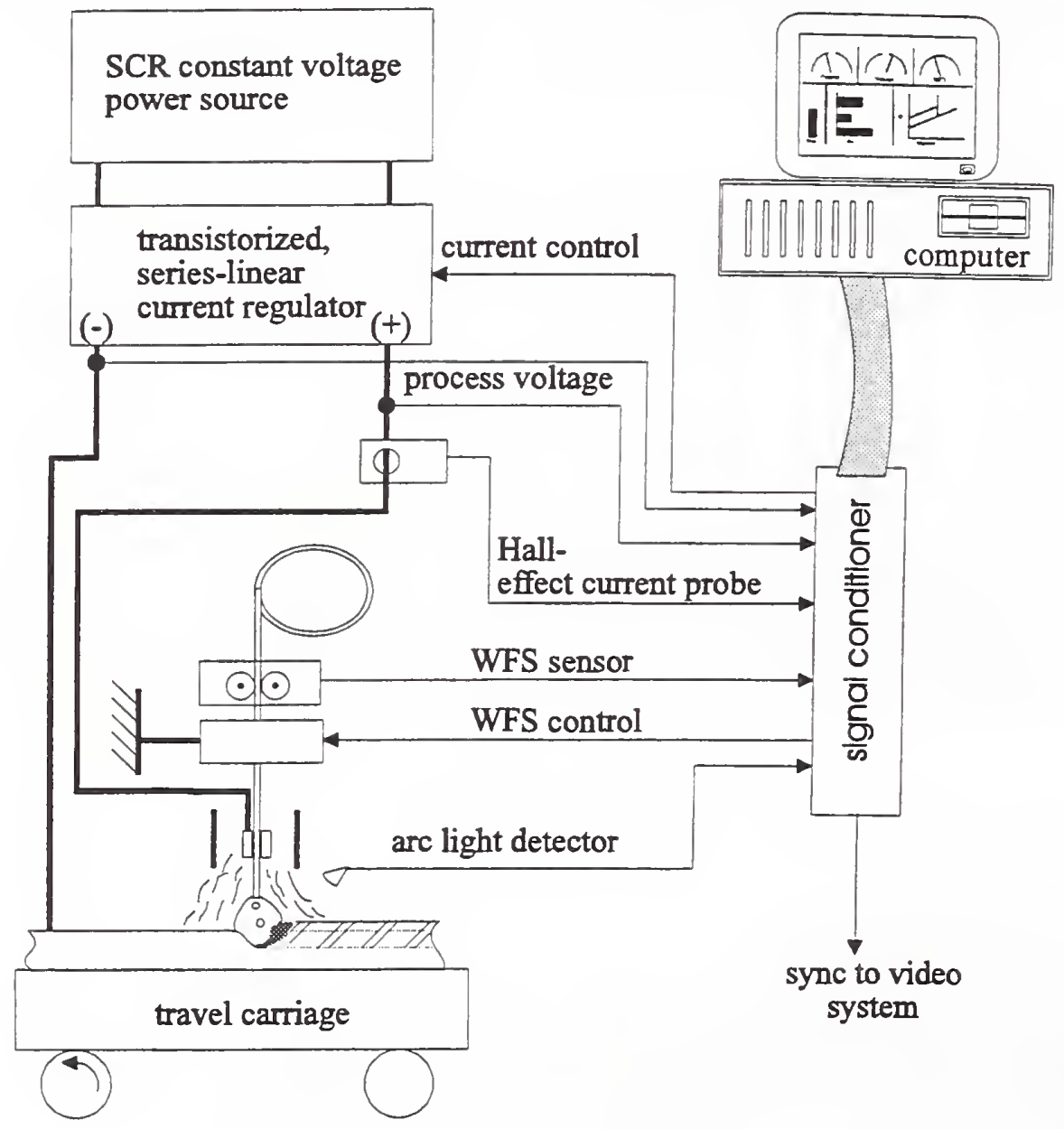

Figure 23. Welding equipment and instrumentation diagram. The welding equipment included a SCR-type power source, a transistorized current regulator, a capstan wire feeder, and a welding torch. The instrumentation included a data-acquisition computer, Hall-effect current sensor, WFS sensor, voltage sensor, and photodiode arclight detector. Both the current regulator and wire feeder were controlled by the computer. 


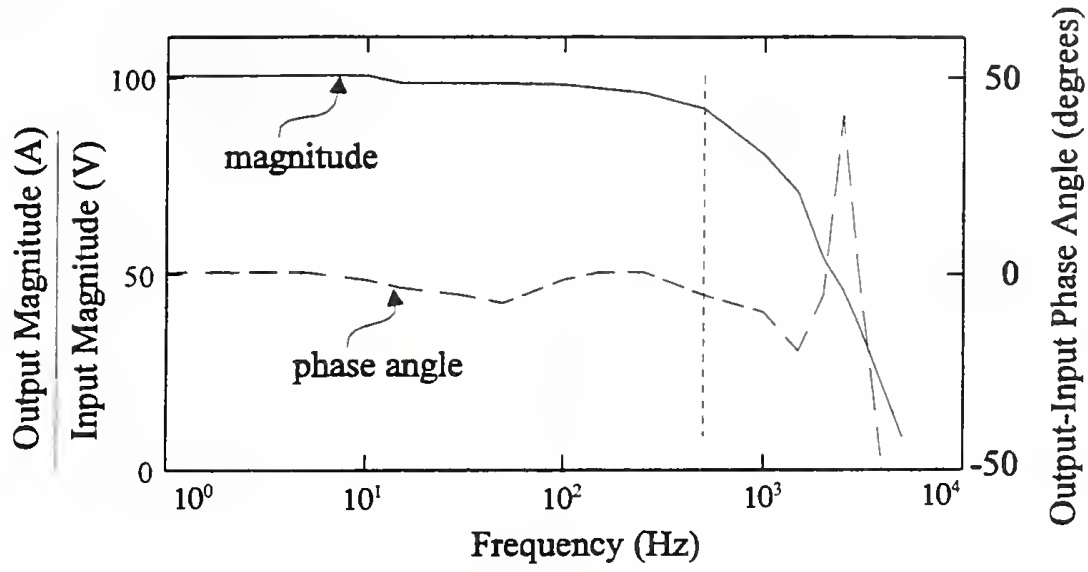

Figure 24. Magnitude and phase-angle frequency-response curves of the current regulator. The regulator was capable of tracking the input signal at welding-current frequencies up to $500 \mathrm{~Hz}$.

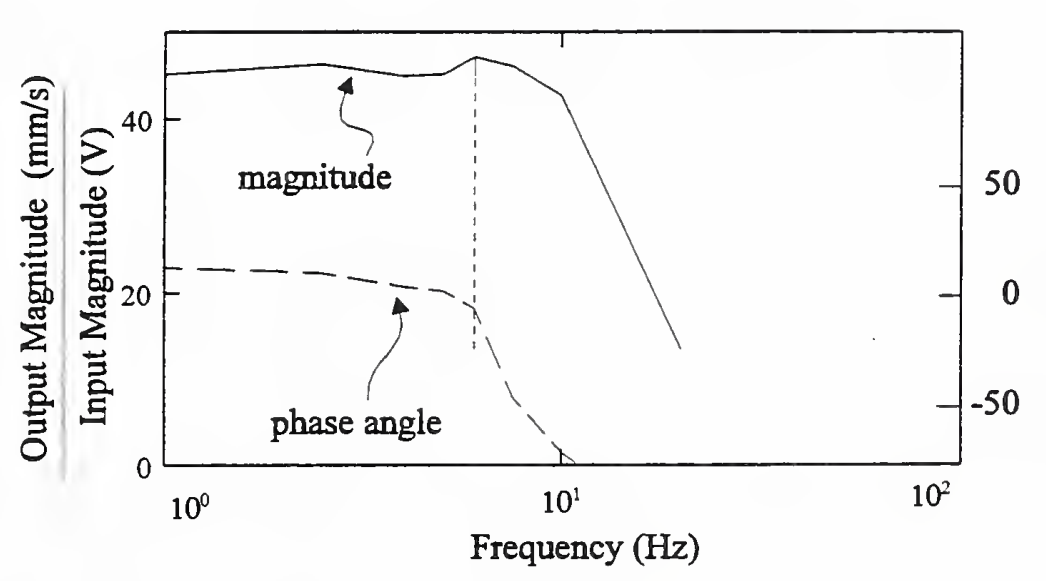

Figure 25. Magnitude and phase-angle frequency-response curves of the wire feeder. The wire feeder was capable of tracking the input signal at WFS frequencies up to $7 \mathrm{~Hz}$.

Arc light was measured with a commercially available, silicon photodiode ${ }^{*}$ that provided an output voltage proportional to incident-light intensity. The photodiode, hereafter referred to as the detector, contained an integral amplifier with a resistoradjustable gain. The spectral response of the detector (shown in fig. 26) ranged from 185 to $1150 \mathrm{~nm}$, with a peak response at $900 \mathrm{~nm}$; the effective spectral response was modified by placing optical filters in front of the detector. The circular, active area of the detector was $5.1 \mathrm{~mm}^{2}$, and its field-of-view or active solid angle was $1.4 \mathrm{sr}$. The detector was mounted on an adjustable positioner.

"Model HUV1100BQ operational amplifier/photodiode, EG\&G Optoelectronics. 


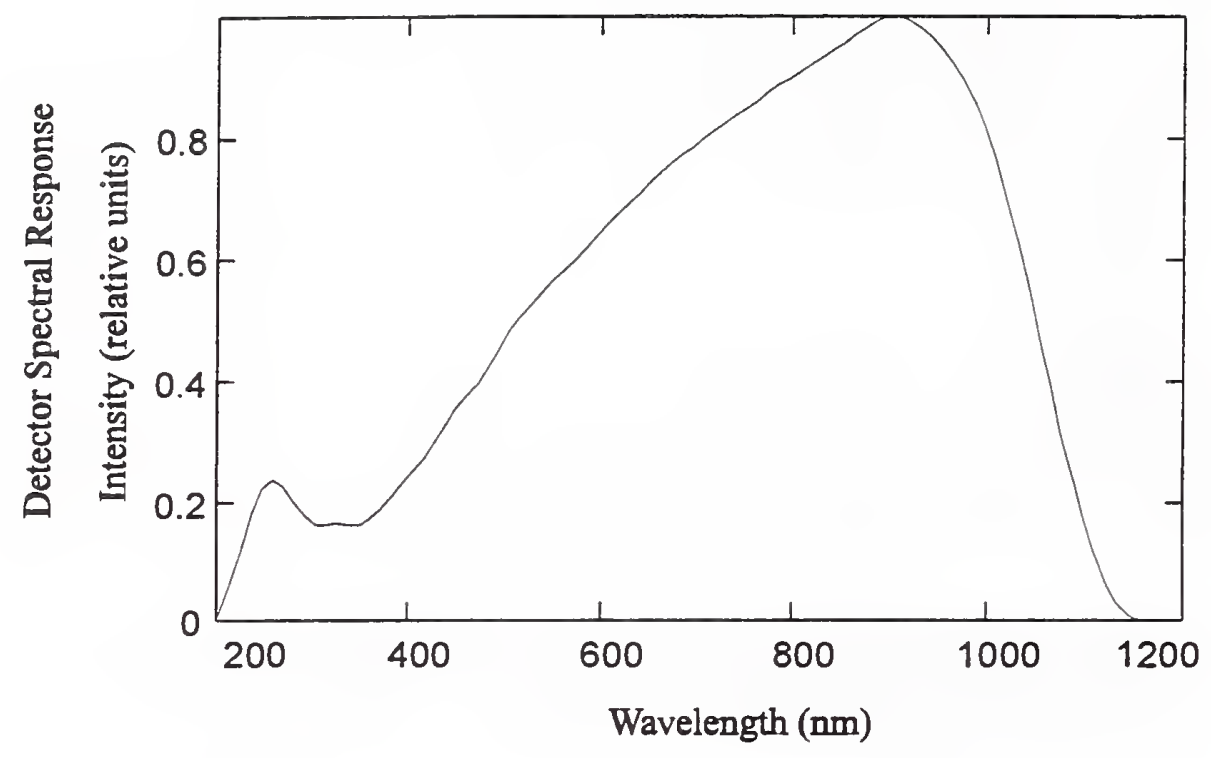

Figure 26. Spectral response of the photodiode (detector) used to measure arc-light intensity. Note that the intensity scale is in relative units. The response of the detector was modified by placing optical filters in front of the detector.

Signals from the current, process voltage, WFS transducer, and detector were isolated and filtered by an analog-signal-conditioning manifold. The signals were sampled by the data-acquisition computer fitted with a multichannel, 12-bit analog-todigital converter (ADC).

A high-speed video system, illustrated in figure 27, recorded images of the welding area. A total of 30000 video frames could be recorded at up to 1000 full or 6000 split frames per second. During data collection, the video system and computer were synchronized by a digital electronic link. A video-frame digitizer and imageanalysis software in the data-acquisition computer provided off-line analysis of the video images. Details of the image processing to extract arc length and droplet transfer frequency are included in sections 5 and 7, respectively. The measurements obtained from the image analysis were then combined and correlated with the data taken from the process transducers.

The video camera was equipped with an adjustable zoom lens. The camera was focused either directly on the arc when using neutral-density (ND) filtering, or on a frosted-glass screen when using the laser backlighting system depicted in figure 27. The backlighting system [42] consisted of a 10-mW He-Ne laser beam and laser line filter. The laser beam was collimated to a diameter of $50 \mathrm{~mm}$ and reflected through the welding area by a beam-bending mirror. The mirror enabled the laser cavity to be kept well away from welding spatter. The laser line filter rejected all arc light, except that at the 632-nm wavelength of the laser. The imaging system produced a highcontrast shadowgraph image of the contact tube, electrode extension, and weld-pool surface on the frosted-glass screen. 


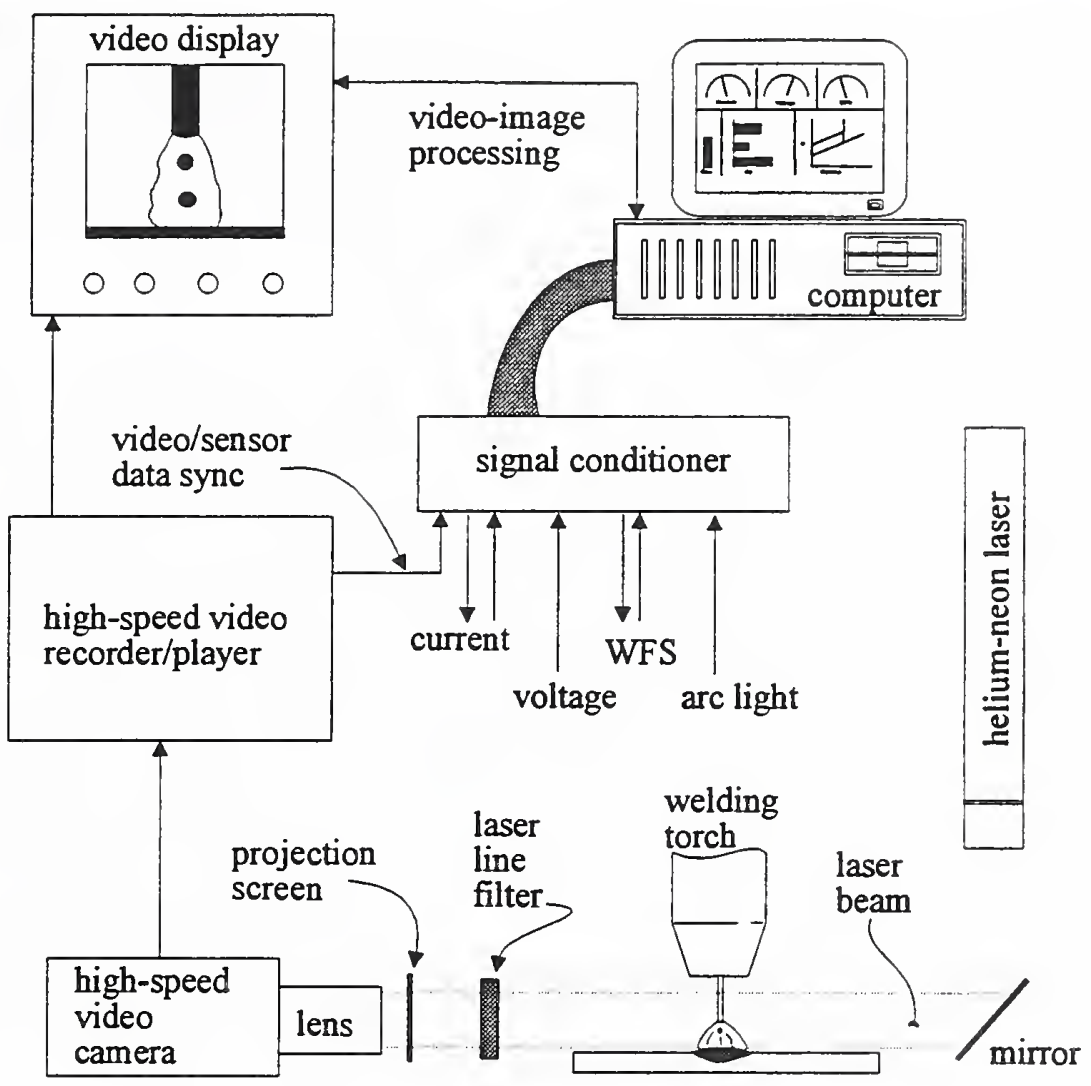

Figure 27. Diagram of the high-speed-video and laser-illumination system diagram. Images were analyzed to extract CTWD, electrode extension, and arc length. The video-image collection was synchronized with the sensor data via an electronic link between the video recorder/player and the data-acquisition computer.

\section{Estimation of Arc Length}

\subsection{Background}

A number of methods for estimating the arc length during GMAW have been developed. They can be divided into two broad classifications: those relying on signals inherent to the process, called through-the-arc sensors, and those using external sensors to obtain information not inherent to the process. Arc-light sensing is an external sensor.

\subsubsection{Through-the-Arc Sensing}

Through-the-arc sensing uses voltage and current sensors with the possible addition of a WFS sensor. An obvious benefit of this technique is that the sensors are located well away from the welding area. Arc current can be measured at practically any location within the welding power circuit. Similarly, process voltage can be measured across the power-source output or, closer to the weld area, across the welding torch and workpiece. The WFS can be measured anywhere along the electrode path. 
The location of the sensor affects the measured process voltage. Measurements across the power-source output include the voltage drops along the power conductors; at the contact-tube/electrode interface; at the electrode extension; between the anode, column, and cathode; and across the workpiece (see fig. 4). Although moving the voltage-sensing points to the torch and workpiece eliminates the drop across the power conductors, the other voltage drops remain. Techniques have been devised to account for voltage drops not associated with the arc itself.

The simplest approach to estimating and subsequently controlling arc length is to assume that voltage drops not associated with the arc are negligible or constant; that is, any change in the process voltage drop is due to changes in the arc voltage [43]. By using a constant-current power source and a wire-feed motor driven by the process voltage, the arc length is initially set by fixing the arc current. The arc length is then maintained by changes in WFS brought on by variations in the process ( $\operatorname{arc}$ ) voltage. This technique is relatively simple, but changes in the process voltage not associated with the true arc voltage can result in variations in arc length.

The most common method of estimating and maintaining arc length is the arcself-regulation technique [43]. A constant-voltage power source and fixed WFS are used. Again, the voltage drops not associated with the arc are assumed to be negligible or constant, and additionally, arc voltage is assumed to be independent of arc current. Here, the arc length is initially adjusted by fixing the power-source voltage and thereafter is maintained by changes in arc current brought on by variations in the process (arc) voltage drop. (Arc length is proportional to arc resistance.) This approach is simple, but because the power-source voltage is not truly constant (that is, it has a finite, negative slope with current), the arc length varies with it.

A more elaborate method for sensing arc length involves modeling the voltage drops across the electrode extension, the arc column, and the anode and cathode regions [44]. The technique requires measurement of arc current, process voltage, and WFS. First, under a steady-state condition where the electrode melting rate is equal to the WFS, the electrode extension $\ell_{e}$ is estimated from the Lesnewich eq (4):

$$
\mathrm{WFS}=\mathrm{a} I_{A}+\mathrm{b} I_{A}^{2} \ell_{e},
$$

where $\mathrm{a}$ and $\mathrm{b}$ are constants for the given electrode and shielding-gas compositions. By rearranging eq (26), $\ell_{e}$ can be obtained in terms of the measurable parameters $I_{A}$ and WFS:

$$
\ell_{e}=\frac{\mathrm{WFS}-\mathrm{a} I_{A}}{\mathrm{~b} I_{A}} .
$$

The electrode-extension voltage drop $E_{e}$ is estimated from

$$
E_{e}=\frac{\rho_{e} \ell_{e}}{A_{e}} I_{A},
$$

where $\rho_{e}$ is the electrode resistivity and $A_{e}$ is the electrode cross-sectional area. The electrode resistivity $\rho_{e}$ must be modeled because it is a function of temperature.

The arc voltage drop can then be found from 


$$
E_{A}=E_{P}-E_{e},
$$

where $E_{P}$ is the process voltage drop. Finally, the arc length is estimated from

$$
\ell_{A}=\frac{E_{A}-E_{O}}{\Delta E_{A} / \Delta \ell_{A}},
$$

where $E_{O}$ is the combined anode- and cathode-voltage drop and $\Delta E_{A} / \Delta \ell_{A}$ is the arc-column potential gradient. Both $E_{O}$ and $\Delta E_{A} / \Delta \ell_{A}$ must be determined experimentally for each combination of electrode and shielding-gas compositions.

The the sensors are relatively simple, and they do not intrude into the weld area, but many parameters must be measured or estimated: The electrode extension must be estimated from arc current and WFS under steady-state conditions. The electrode extension voltage must be calculated from electrode resistivity, which is a function of the temperature distribution along the electrode. Appropriate values of the anode and cathode voltage and the arc-column-voltage gradient must be obtained for each combination of electrode and shielding-gas compositions. Also, the technique relies on accurate sensing of WFS.

\subsubsection{External Sensors}

Several techniques have been developed to obtain arc length from sensors external to the process or by a combination of external sensors and through-the-arc sensing.

A weld-torch-proximity sensor together with arc-current and WFS sensors can be used to obtain arc length [45]. The proximity sensor, typically a laser-based triangulation unit mounted to the torch, is used to obtain the CTWD. The electrode extension can be obtained from WFS and current, as described in the previous section [eq (26)]. Electrode extension can also be obtained by an ultrasonic pulse-echo method, where compression waves are generated in the electrode and reflected from its end [46]. Then the arc length can be obtained directly from

$$
\ell_{A}=\mathrm{CTWD}-\ell_{e} .
$$

This technique eliminates several of the assumptions about the process conditions required to obtain the arc length. Also, it eliminates the dependence on processvoltage drop, and the proximity sensor can provide additional information for joint tracking and joint-profile interrogation. The primary disadvantages of the technique include the cost, size, and the intrusiveness of the proximity sensor.

Direct viewing of the welding arc has been used for arc-length measurement [47]. An optical-sensor array, such as a video camera, gives a two-dimensional image of the weld area. The intense arc light provides a high-contrast image from which the boundaries of the arc are readily obtained by image processing. By calibrating the image magnification, the arc length can be extracted directly. An advantage of this technique is that it provides a direct measure of arc length. The technique is independent of welding parameters, such as electrode and shielding-gas compositions and model-parameter estimates. Disadvantages include the cost, size, and the intrusiveness 
of the video camera, as well as the cost and program development of an image processor. Another disadvantage is the need to maintain focused optics near the hostile welding environment.

The approach patented by Johnson and Sciaky in 1966 [48] is simpler because no focusing optics are used to obtain arc-light information. A light guide, attached to the torch, carries the arc light to a photodiode. The voltage from the photodiode is compared with a reference voltage, and the difference between the two (the error signal) is used to drive a controller that actuates WFS to maintain constant arc-light intensity. The system has not been a commercial success however, possibly because it is unable to discriminate changes in arc-light intensity due to variations in arc length from those due to changes in arc current. A similar system for GTAW was patented by Sciaky and Vilkas, also in 1966 [49].

Another arc-length sensor based on arc-light intensity was developed by Romanenkov in 1976 for low-current GTAW ( $\sim 10$ to $30 \mathrm{~A}$ ), where voltage sensing for arc-length control is not reliable [50]. His study is one of the few that address specific regions of the arc spectrum that can be exploited for arc-length sensing. He determined that the intensity obtained from a broad spectrum of arc light is not stable as the welding parameters change. By using several wavelength regions, including $\mathrm{Ar}$ lines and bands of the continuum, the arc length could be estimated within $0.1 \mathrm{~mm}$.

More recently, in 1989, Deam and Drew investigated the relationship between arc-light intensity, arc current, and arc length in GTAW [51]. An optical-light detector, covered by a $550-\mathrm{nm}$ narrow-band filter and located $200 \mathrm{~mm}$ from the arc, was used to obtain an arc-light-intensity signal. For currents in the range 90 to $200 \mathrm{~A}$, the light signal, $L$, was related to current and arc length by

$$
L=\mathrm{k} \ell_{A} I_{A}^{1.5},
$$

where $\mathrm{k}$ is a proportionality constant. The arc-length resolution of the sensor was not reported.

\subsubsection{Review of Preliminary Work}

The technique for estimating arc length developed here has its origin in a program designed to investigate alternative sensors-for example, electric field, vibration, and optical-for control of GMAW [52]. As a result of that work, an empirical model relating arc-light intensity to arc length and current in GMAW was developed [53]. Arc-light-intensity data were obtained from a photodiode detector with ND filtering. Actual arc length and arc current were measured and correlated to arc-light intensity. Inspection of the arc-light and arc-length data at constant arc current suggested that the relationship between light intensity and arc length can be modeled as

$$
E_{D}=\mathrm{C}_{0}+m \ell_{A}
$$

where $E_{D}$ is the detector voltage, $C_{0}$ represents the detector voltage at $\ell_{A}=0$, and $m$ is the detector voltage/arc length slope. Inspection of the arc-light and current data at constant arc lengths indicated $m$ can be modeled by

$$
m=\mathrm{C}_{1}+\mathrm{C}_{2} I_{A}
$$


where $C_{1}$ is an offset constant and $C_{2}$ is the change in detector voltage with current at constant arc length. Substituting eq (34) into eq (33) yields

$$
E_{D}=\mathrm{C}_{0}+\left(\mathrm{C}_{1}+\mathrm{C}_{2} I_{A}\right) \ell_{A}
$$

Expanding eq (35) gives

$$
E_{D}=\mathrm{C}_{0}+\mathrm{C}_{1} \ell_{A}+\mathrm{C}_{2} I_{A} \ell_{A}
$$

Thus, the arc-light intensity is proportional to arc length and the product of arc length and current. The correlation of the arc-length, current, and light-intensity data with the empirical model enabled arc length to be estimated within $1 \mathrm{~mm}$ [53].

\subsection{Arc-Length Sensor}

As indicated in the previous section, the functional relationship initially developed for the arc-length sensor was based on inspection of the current, voltage, and arc-length data. Here, the relationship is formulated by a more fundamental analysis of arc characteristics.

From work conducted with GTAW, Alum [39] concluded that the power lost from the arc column by radiation must be proportional to the voltage drop across the arc column and the current flowing through it:

$$
P_{L}=E_{A} I_{A} \beta \text {, }
$$

where $\beta$ is the fraction of arc power lost by radiation and $P_{L}$ is the arc radiant power.

An expression for the arc voltage is derived by a linear combination of eq (9) and eq (10)

$$
E_{A}=\frac{\zeta_{0}}{I_{A}}+\zeta_{1} \ell_{A}+\zeta_{2} I_{A}
$$

Equation (38) accounts for the increase in arc voltage with both length and current. Substitution of eq (38) into eq (37) yields

$$
P_{L}=\beta \zeta_{0}+\beta \zeta_{1} \ell_{A} I_{A}+\beta \zeta_{2} I_{A}^{2}
$$

In section 3, the detector voltage $E_{D}$ as a function of the light intensity from the arc was developed [eq (25)] and is repeated here:

$$
E_{D}=\alpha P_{L} \cos \theta_{D}
$$

An expression for the detector voltage as a function of arc length and current can be obtained by substituting eq (39) into eq (40):

$$
E_{D}=\alpha \beta \zeta_{0} \cos \theta_{D}+\alpha \beta \zeta_{1} \cos \theta_{D} \ell_{A} I_{A}+\alpha \beta \zeta_{2} \cos \theta_{D} I_{A}^{2}
$$


During welding, the detector angle $\theta_{D}$ is fixed and, therefore, $\cos \theta_{D}$ is a constant. As a result, each group of constants $\alpha \beta \zeta_{i} \cos \theta_{D}$ can be replaced by a single constant $C_{i}$ :

$$
E_{D}=\mathrm{C}_{0}+\mathrm{C}_{1} \ell_{A} I_{A}+\mathrm{C}_{2} I_{A}^{2}
$$

To estimate the arc length, eq (42) is solved for $\ell_{A}$ :

$$
\ell_{A}=\frac{E_{D}-\left(\mathrm{C}_{0}+\mathrm{C}_{2} I_{A}^{2}\right)}{\mathrm{C}_{1} I_{A}} .
$$

Equation (43) represents the arc-length model based on the readily measured process parameters arc-light intensity (detector voltage) and arc current. References to the arclength "sensor" embody eq (43).

\subsection{Detector}

For arc-length estimation, it is important to sense radiation from the arc column itself and to minimize radiation from sources, such as the weld pool and electrode tip, that are independent of arc length. Proper selection of optical filters for the detector, as well as detector orientation and location relative to the arc, results in optimum arclength estimation.

\subsubsection{Location and Orientation}

The influence of detector location and orientation on detector output is described by the radiant-energy-transfer equation, eq (19). The source/detector configuration is shown in figure 28. A coordinate system is positioned at the center of the weld pool with the $y$-axis along the direction of travel. The center of the detector is given by the line-of-sight vector $\mathbf{r}$, and the plane of the detector has a normal vector $\mathbf{n}$. The magnitude of $\mathbf{r}$ is $R_{D}$. The angle between $\mathbf{r}$ and the $z$-axis is given by $\theta_{s}$; the angle between $\mathbf{r}$ and $\mathbf{n}$ is $\theta_{D}$; and the angle between the projection of $\mathbf{r}$ in the $x-y$ plane and the direction of travel $(y)$ is $\theta_{z}$. The weld pool, arc, and electrode tip are assumed to be within the field of view of the detector.

For radiation from the weld pool and electrode tip, the equation for radiantenergy transfer takes the following form (see section 3.5):

$$
E_{D}=\mathrm{k} \frac{L_{S} A_{S} \cos \theta_{S} A_{D} \cos \theta_{D}}{R_{D}^{2}},
$$

where $\mathrm{k}$ is a proportionality constant. Assume constant values for all parameters except $\theta_{S}$. For radiation from the weld pool, which is assumed to be a planar source, the detector voltage $E_{D}$ increases as the angle of the detector relative to the pool $\theta_{S}$ decreases. Thus, to minimize sensitivity to pool radiation, $\theta_{S}$ should be a maximum. For radiation from the molten electrode tip, the tip is assumed to be a spherical source. The detector voltage is independent of $\theta_{S}$ (from the spherical source assumption); therefore, radiation received from the electrode tip is constant and cannot be minimized. Typically, the electrode-tip area is much smaller than the weld-pool area, and the radiation from the electrode is less than that from the pool. 


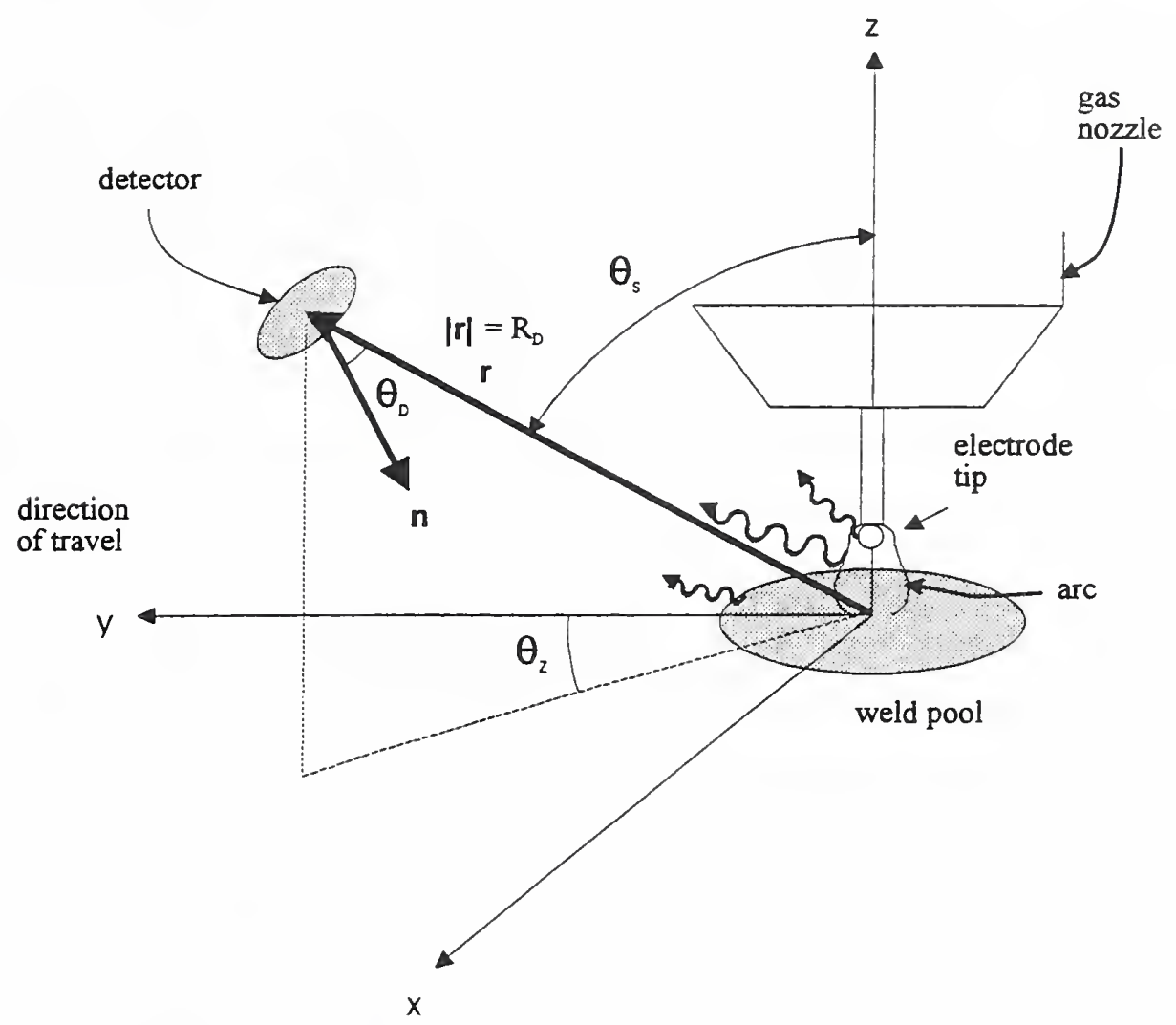

Figure 28. The source/detector geometric configuration. The center of the detector relative to the weld pool is given by the line-of-sight vector $r$ and the plane of the detector has a normal vector $\mathbf{n}$.

Treating the arc as a volume source (see section 3.5) that radiates isotropically in all directions, the radiant-energy-transfer equation for the arc is

$$
E_{D}=\frac{\alpha L_{A} V_{A} A_{D} \cos \theta_{D}}{R_{D}^{2}} .
$$

Assuming that the arc is an isotropic volume source eliminates dependence of the arc on $\theta_{S}$. We expect, however, that as the detector angle decreases, the detector will receive a greater portion of any arc light reflected by the pool and workpiece. Therefore, the detector angle $\theta_{S}$ relative to the pool and workpiece should again be as close to $90^{\circ}$ as possible. Figure 28 also shows that, below a particular value of $\theta_{S}$, the gas nozzle would begin to block part of the radiation from the arc.

The orientation of the detector relative to the line-of-sight axis is described by the angle $\theta_{D}$, as defined in figure 28. To receive maximum output from the detector, it should be oriented such that $\mathbf{n}$ is coincident with the line-of-sight vector $\mathbf{r}$. The assumptions that the arc is a volume source and that it is symmetric about the electrode wire axis indicate that the detector output is independent of $\theta_{Z}$. However, the weld 
reinforcement might block a portion of the arc light when $\theta_{Z}$ is near $180^{\circ}$ and $\theta_{S}$ is large.

The minimum distance between the detector and the source is limited by the constraint that the field of view for the detector must include all of the arc. The detector field of view is $1.4 \mathrm{sr}$ and the solid angle is denoted by $\Omega_{D}$. The arc is assumed to project a circular area $A_{S}$ with a diameter (arc length) of $20 \mathrm{~mm}$. The minimum distance between the arc and the detector $R_{D}$ can be estimated by [41]

$$
R_{D} \approx\left(\frac{A_{S}}{\Omega_{D}}\right)^{\frac{1}{2}}=\left[\frac{\pi\left(\frac{20 \mathrm{~mm}}{2}\right)^{2}}{1.4 \mathrm{sr}}\right]^{\frac{1}{2}}=15 \mathrm{~mm}
$$

The detector can be placed at any distance greater than that calculated above. Equation (45) shows that the detector voltage is inversely proportional to $R_{D}^{2}$. Within practical limits, the addition or deletion of ND filtering and/or the adjustment of detector gain can be used to maintain the detector output within the voltage range of the recording device as $R_{D}$ varies.

\subsubsection{Spectral Locations for Sensing}

To select filters for modifying the detector response, two criteria were considered simultaneously: the emission spectrum of the arc and the band-pass characteristics of readily available optical filters. Optical band-pass filters are characterized by the center wavelength and half-power bandwidth. The center wavelength is the wavelength of maximum transmittance. The half-power bandwidth is the width of the band pass about the center wavelength, where the transmittance has been reduced to half that at the center wavelength. Of the few detailed sources available for actual weldingarc spectra, that by Peterson [33] is central to the present topic. He cataloged the visible arc spectrum during GMAW of steel using $98 \% \mathrm{Ar}+2 \% \mathrm{O}_{2}$ (see fig. 16). That spectrum is used to select spectral locations, and therefore optical filters, for optimal arc-length sensing.

Only a ND filter was used for evaluation because it was the only filter used in the preliminary work [53], and therefore it provides a basis for determining any improvement resulting from the use of narrow-band-pass filters. The ND filter attenuates all wavelengths equally; therefore, the spectral response is that of the detector itself. Figure 29 shows the response of the detector, the known emission spectrum of the arc, and the theoretical blackbody emission from the weld pool and electrode tip as calculated from Planck's radiation law [53]. Note that the curves for detector sensitivity and emissions from the arc, pool, and electrode are not scaled to each other; only the change in relative magnitude within each trace is represented. The detector is most sensitive in the near-infrared region, where blackbody radiation has the greatest intensity. The use of a ND filter provides the greatest sensitivity to arc-length-independent radiation from the weld pool and electrode tip.

To reduce the effects of emissions from the pool and electrode, a blue-bandpass (BBP) filter was selected. This filter has a center wavelength at $430 \mathrm{~nm}$ and a half-power bandwidth of $140 \mathrm{~nm}$. Figure 30 shows the transmittance profile of the filter along with the available arc and theoretical blackbody emission spectra. The 


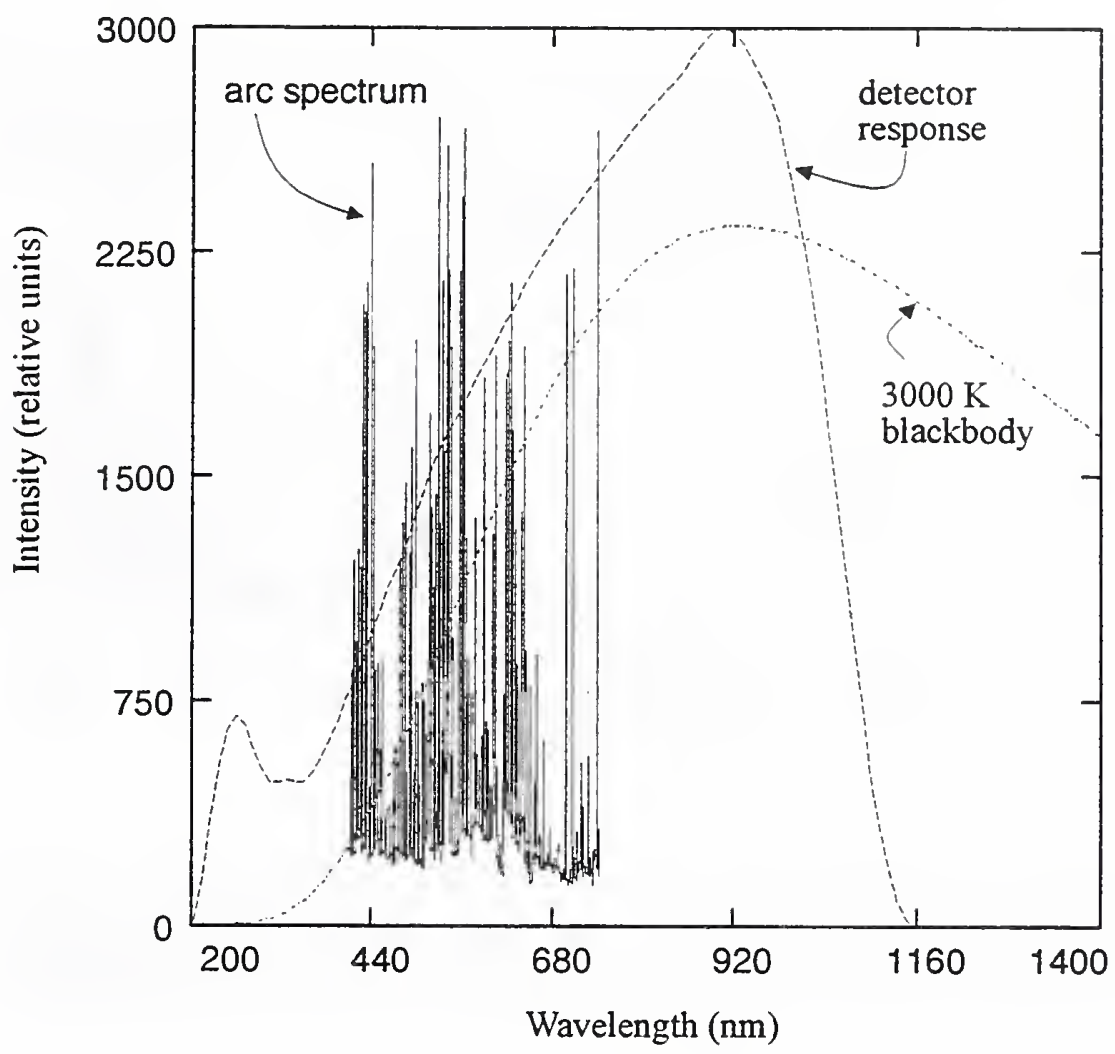

Figure 29. The arc and blackbody spectra and the detector-response profile. With ND filtering, the response of the detector is a maximum in the near-infrared and extends beyond both ends of the available arc spectrum. Note that the traces are not scaled to each other; only the change in relative magnitude within each trace is represented.

filter attenuates the blackbody radiation and the longer (red) wavelengths from the arc. The filter transmits the shorter (blue) wavelengths consisting of Fe lines as well as uncharted near-ultraviolet radiation from the arc.

Based on the work of Romanenkov [50], regions of the arc spectrum associated with emissions from specific species in the arc as well as a region of the continuum were selected for arc-length sensing. For the welding of steel with an Ar-based shielding gas, $\mathrm{Fe}$ and Ar lines were selected as candidates for evaluation. Scanning the arc spectrum revealed a group of Fe lines clustered around $430 \mathrm{~nm}$, a single Ar line at $696.5 \mathrm{~nm}$, and a region of continuum extending from 454 to $470 \mathrm{~nm}$.

Figure 31 shows the filter transmission profile for a $430-\mathrm{nm}$ filter with a halfpower bandwidth of $10 \mathrm{~nm}$ and the associated arc spectrum. Except for the lines noted, the arc spectrum consists of Fe lines. The available filter closest to the 695-nm Ar line had a center wavelength at $694 \mathrm{~nm}$ with a 10-nm half-power bandwidth. The arc spectrum and filter characteristic for the 694-nm filter are shown in figure 32. A narrow-band filter with a center wavelength of $467 \mathrm{~nm}$ was used for evaluating the continuum region (fig. 33). 


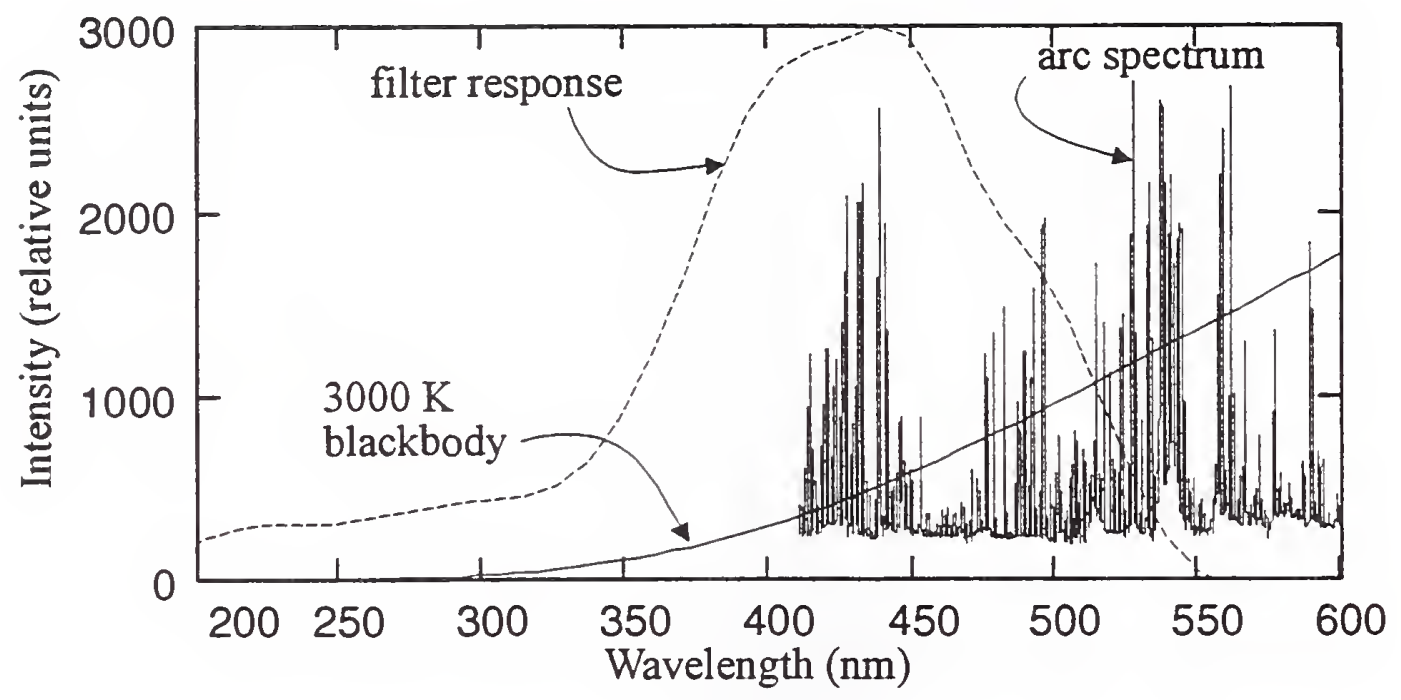

Figure 30. Blue-band-pass (BBP) optical-filter transmission profile and arc and blackbody spectra. The filter attenuates the blackbody radiation and transmits a wide range of arc radiation. The arc spectrum in the passband consists primarily of Fe lines. The arc spectrum is from Peterson [33].

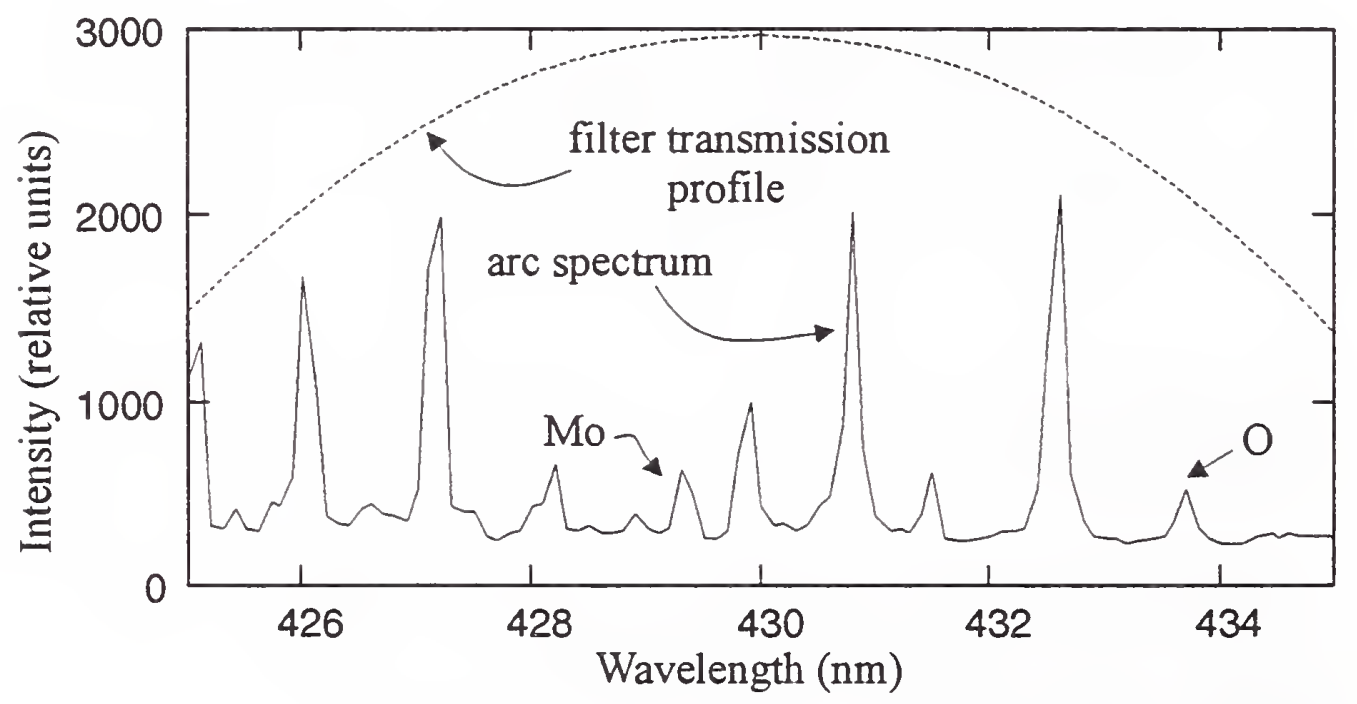

Figure 31. Filter-transmission profile for a $430-\mathrm{nm}$ filter with a halfpower bandwidth of $10 \mathrm{~nm}$ and the associated arc spectrum. Except for the lines noted, the arc spectrum consists of Fe lines. The arc spectrum is from Peterson [33]. 


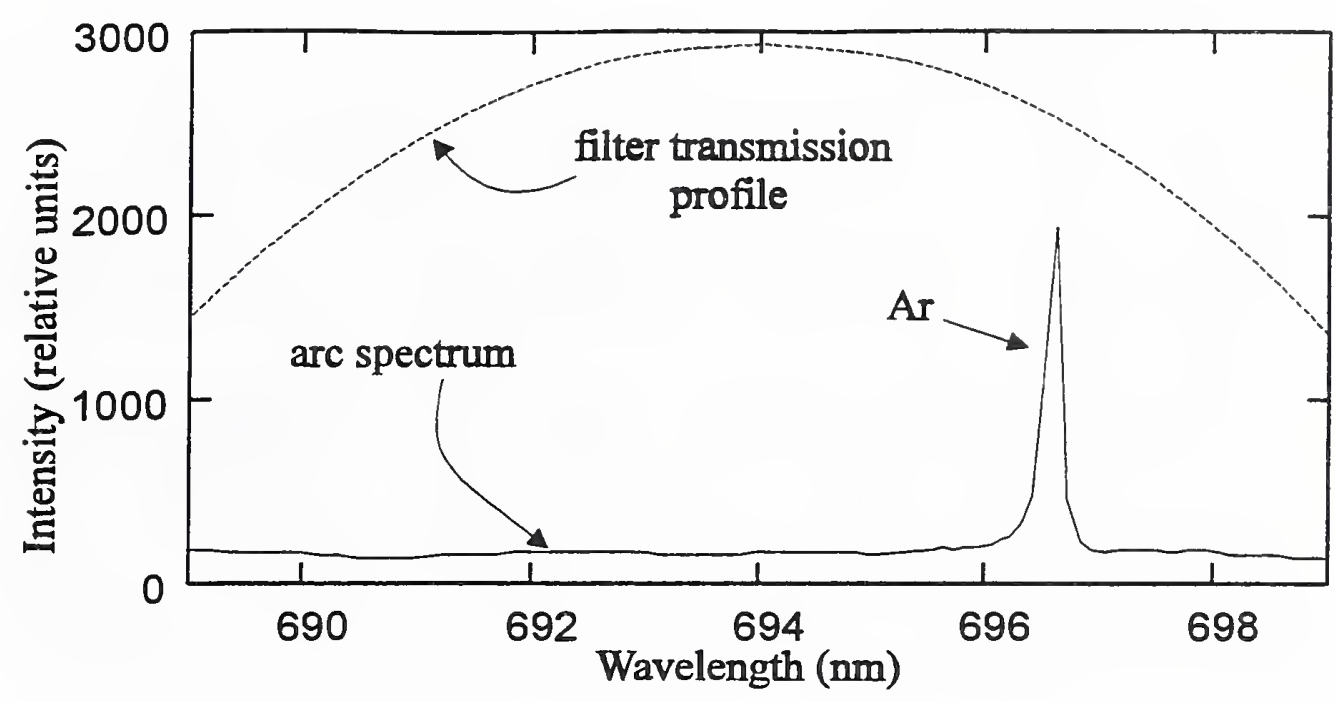

Figure 32. A 694-nm optical-filter-transmission profile and arc spectrum. The arc spectrum consists primarily of the continuum with a single Ar line at $696.5 \mathrm{~nm}$. The arc spectrum is from Peterson [33].

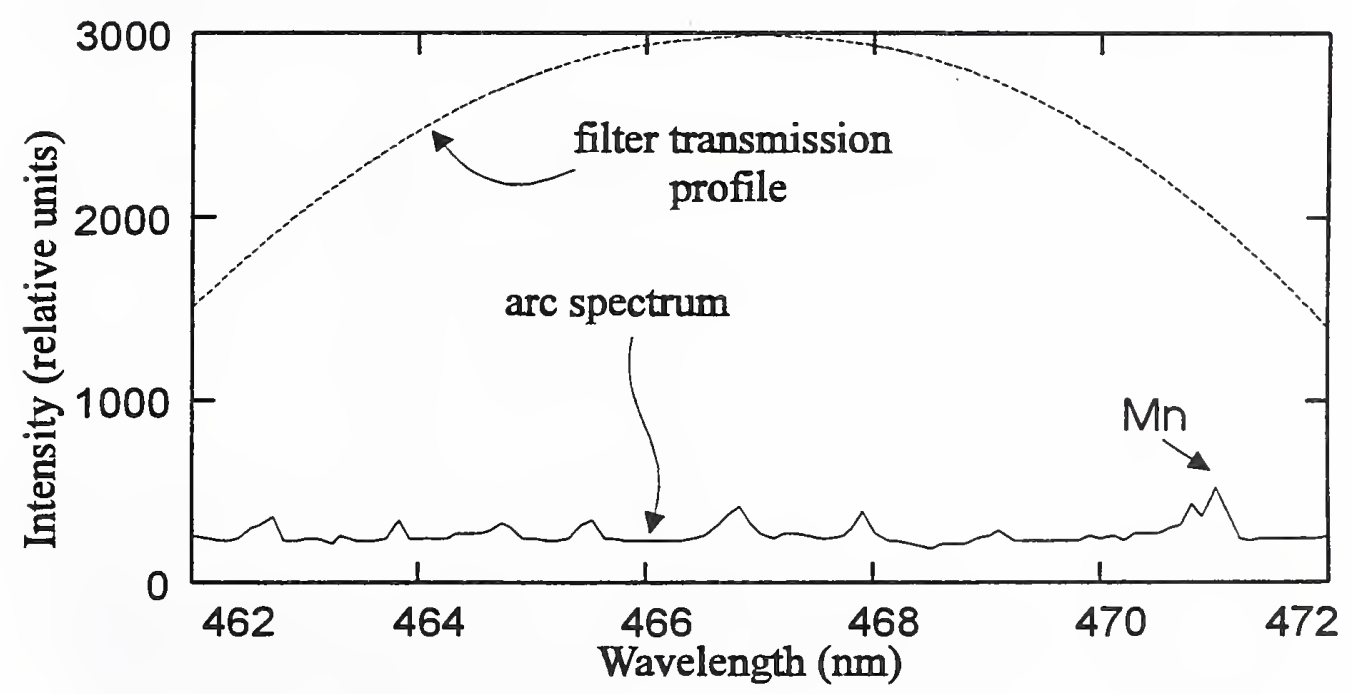

Figure 33. A 467-nm optical-filter-transmission profile and arc spectrum. The arc spectrum consists primarily of the continuum with an $\mathrm{Mn}$ line at the longer wavelengths. The arc spectrum is from Peterson [33]. 


\subsection{Experimental Method}

\subsubsection{Welding Procedure}

Bead-on-plate welds were completed on AISI-1020-steel strips with an ER100S-1*-steel electrode and a 19-mm CTWD. The welds were made with either $98 \% \mathrm{Ar}+2 \% \mathrm{O}_{2}$ or $95 \% \mathrm{Ar}+5 \% \mathrm{CO}_{2}$ as the shielding gas. The travel speed was set at $6 \mathrm{~mm} / \mathrm{s}$; about $30 \mathrm{~s}$ was required to make each weld. The detector orientation and distance relative to the arc and optical filtering remained fixed during each weld.

To cover a large range of welding conditions for each combination of shielding gas, optical filter, and detector position, the current and WFS were varied during each weld. The current was modulated with a triangular waveform at $25 \mathrm{~Hz}$ about a mean current $(\sim 240 \mathrm{~A})$ providing spray transfer. The amplitude of current modulation was $60 \mathrm{~A}$, providing a minimum current of $210 \mathrm{~A}$ and a maximum of $270 \mathrm{~A}$. The currentmodulation frequency was set high enough to prevent changes in the electrode melting rate with current, or in other words, to maintain constant arc length and electrode extension for the given WFS [54]. The WFS was incremented in 20 steps over the length of the weld from about 110 to $145 \mathrm{~mm} / \mathrm{s}$. The change in WFS resulted in arc lengths from about 15 to $3 \mathrm{~mm}$ over the range of arc current.

\subsubsection{Detector Configuration}

The detector was mounted on an adjustable arm so that its distance and orientation relative to the arc could be changed. For all welds, $\theta_{Z}$ was fixed at $15^{\circ}$. The optical filters were supported in front of the detector by the detector enclosure and could be readily changed.

To determine the effect of sensor orientation on detector output, two sets of welds were made. First, the detector/source angle $\theta_{S}$ was varied from $85^{\circ}$ to $60^{\circ}$ in $5^{\circ}$ increments while $\theta_{D}$ was held at $0^{\circ}$ (see fig. 28). A maximum of $85^{\circ}$ was selected to maintain the detector above the top surface of the workpiece. Next, $\theta_{D}$ was varied from $0^{\circ}$ to $25^{\circ}$ in $5^{\circ}$ increments while $\theta_{S}$ was fixed at $85^{\circ}$. For both of these conditions, the detector-to-arc distance, $R_{D}$, was held constant at $400 \mathrm{~mm}$. A weld was made for each combination of shielding gas and optical filter.

To determine the effect of detector distance, $R_{D}$ was varied from $200 \mathrm{~mm}$ to $600 \mathrm{~mm}$ in $100-\mathrm{mm}$ increments. For these welds, $\theta_{D}$ was held at $0^{\circ}$ and $\theta_{S}$ was fixed at $85^{\circ}$. Distances less than $200 \mathrm{~mm}$ were not practical owing to torch and workpiece fixturing; distances greater than $600 \mathrm{~mm}$ could not be easily accommodated by the detector arm. Although both shielding gases were used, only the BBP optical filter was evaluated.

Once the optimum detector orientation and distance were determined, the detector position was fixed, and additional welds were made with each shielding gas and optical filter combination for final performance evaluation.

\footnotetext{
*Designation of the American Welding Society.
} 


\subsubsection{Data Collection and Analysis}

While each weld was made, the computer collected values for arc current and voltage, WFS, and detector voltage at $500 \mathrm{samples} / \mathrm{s}$. Simultaneously, video images were collected at 500 frames/s.

After each weld, the video images were processed to obtain measures of CTWD, electrode extension, and arc length for each corresponding value of current, voltage, WFS, and detector voltage. The video camera lens was adjusted to obtain an image including the end of the contact tube, electrode extension, arc, and the top of the weld pool. The magnified images had a resolution of $0.08 \mathrm{~mm} / \mathrm{pixel}$. Figure 34 shows video images from different welding conditions and the method for measuring arc length. A horizontal video line was set at the end of the contact tube as the reference. Three video lines were scanned parallel to and within the electrode region to find the end of the electrode. The electrode extension was defined as the average length of the three lines extending from the reference line at the contact tube to the end of the electrode. Starting at an arbitrary position in the workpiece region, seven

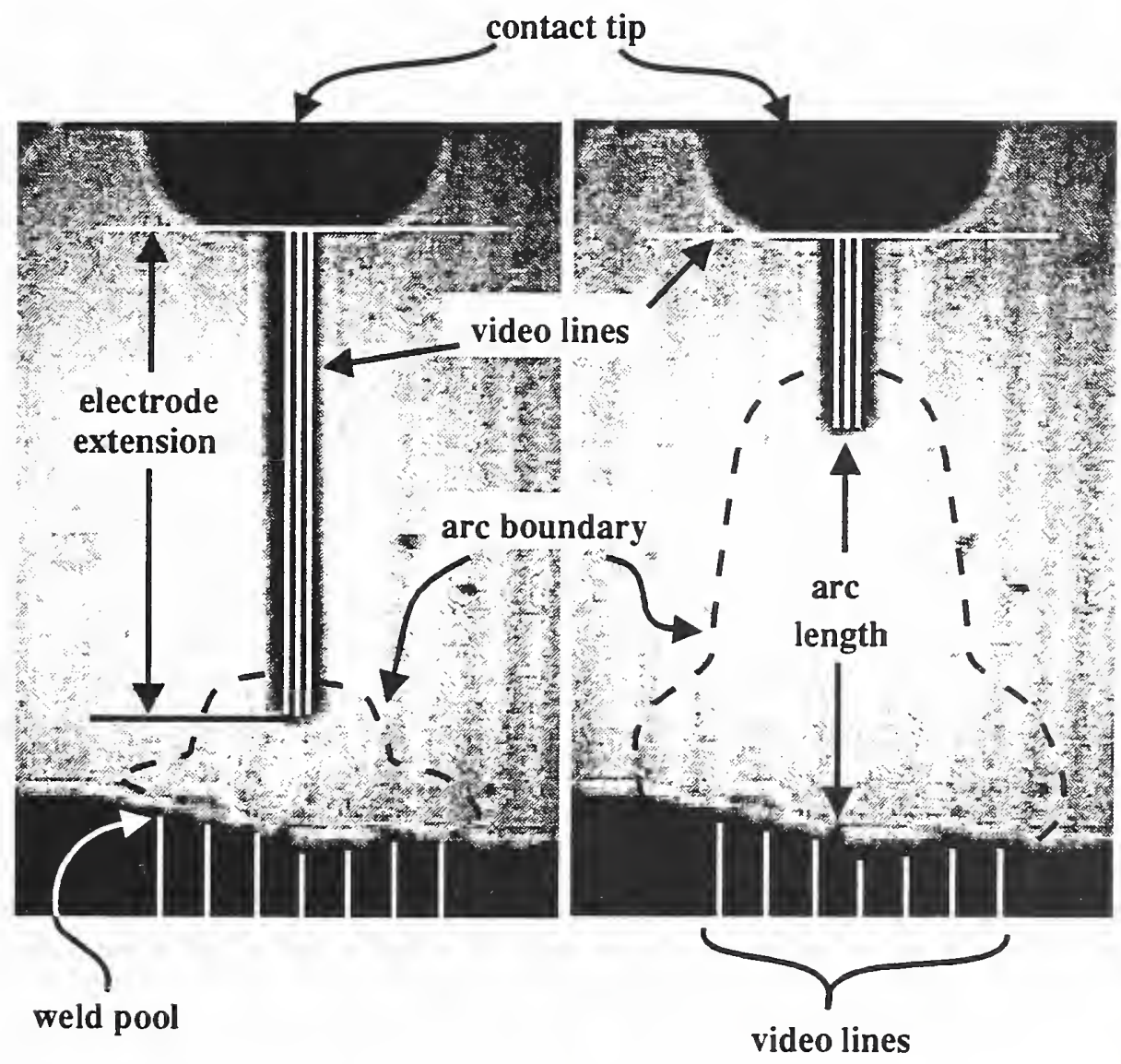

Figure 34. Video images for different welding conditions processed to obtain the arc length. The arc length is defined as the difference between the CTWD and the electrode extension. 
video lines were scanned to find the top surface of the weld pool. The CTWD was defined as the distance between the reference line at the contact tube and the average of the seven lines defining the weld-pool surface. The arc length was then found by subtracting the electrode extension from the CTWD. As indicated in figure 34, under most welding conditions, the arc boundary at the electrode extends above the end of the electrode.

A multivariate, linear-regression analysis was performed to fit each data set to eq (42) and to obtain values for the coefficients $C_{0}, C_{1}$, and $C_{2}$. The regression analysis yielded a correlation parameter $R_{c}$ to indicate the goodness of fit as well as the standard deviation for the fit $\sigma_{\mathrm{c}}$. A perfect fit is indicated by $R_{c}=1$; therefore, the closer $R_{c}$ is to 1 , the better the fit. Conversely, a perfect fit is also indicated by $\sigma=0$; thus, the closer $\sigma_{\mathrm{c}}$ is to 0 , the better the fit. Using $R_{c}$ and $\sigma_{c}$ enables a direct comparison of the sensor performance under the different combinations of detector filter, detector position, and shielding gas.

\subsection{Results and Discussion}

\subsubsection{Correlation of Results to the Arc-Length-Sensor Model}

Time traces for the process-input parameters (current, voltage, WFS, and CTWD) for a weld made with a BBP optical filter and $98 \% \mathrm{Ar}+2 \% \mathrm{O}_{2}$ shielding gas are shown in figure 35 . The detector was $400 \mathrm{~mm}$ from the arc, with $\theta_{D}=0^{\circ}$ and $\theta_{S}=80^{\circ}$. Each record represents $30 \mathrm{~s}$ of welding with 15000 data points and 750 current oscillations. The current trace shows that the mean value and modulation amplitude are constant throughout the weld. The voltage trace shows a modulation amplitude analogous to that of the current, but the mean voltage decreases as the WFS increases. The CTWD is nearly constant throughout the weld.

For the same weld, the process-output parameters (electrode extension, arc length, and arc-light-detector voltage) are shown in figure 36 . The electrode extension increases and, correspondingly, the arc length decreases as the WFS increases. The electrode-extension and arc-length traces are not narrow lines but have a range or envelope that is attributed to the cyclic nature of electrode extension change due droplet detachment, natural-process variation, and noise in the video images. In the trace of the arc-light-detector voltage, two trends are obvious: as the arc length decreases, the mean detector voltage decreases, and as the arc length decreases, the envelope or modulation amplitude also decreases.

Figure 37 shows, in greater detail, a section of the waveforms in figure 36. Here, the WFS is $123 \mathrm{~mm} / \mathrm{s}$ and the CTWD is $20 \mathrm{~mm}$. The current record shows the triangular waveform of the input reference. The behaviors of the process and detector voltages are very similar to that of the current. Except for minor variations, the arc length is constant at about $9 \mathrm{~mm}$, as shown in figure 37 .

The coefficients of the arc-length-sensor model [eq (43)] for the data set represented in figures 36 and 37 are $\mathrm{C}_{0}=-0.576 \mathrm{~V}, \mathrm{C}_{1}=1.435 \times 10^{-3} \mathrm{~V} /(\mathrm{A} \cdot \mathrm{mm})$, and $\mathrm{C}_{2}=2.872 \times 10^{-5} \mathrm{~V} / \mathrm{A}^{2}$. Arc length versus detector voltage for several values of current is plotted in figure 38 . The solid lines represent the sensor model; the symbols are actual data points. The detector voltage and, hence, arc-light intensity increase linearly with arc length, and the slope increases with increasing current. 


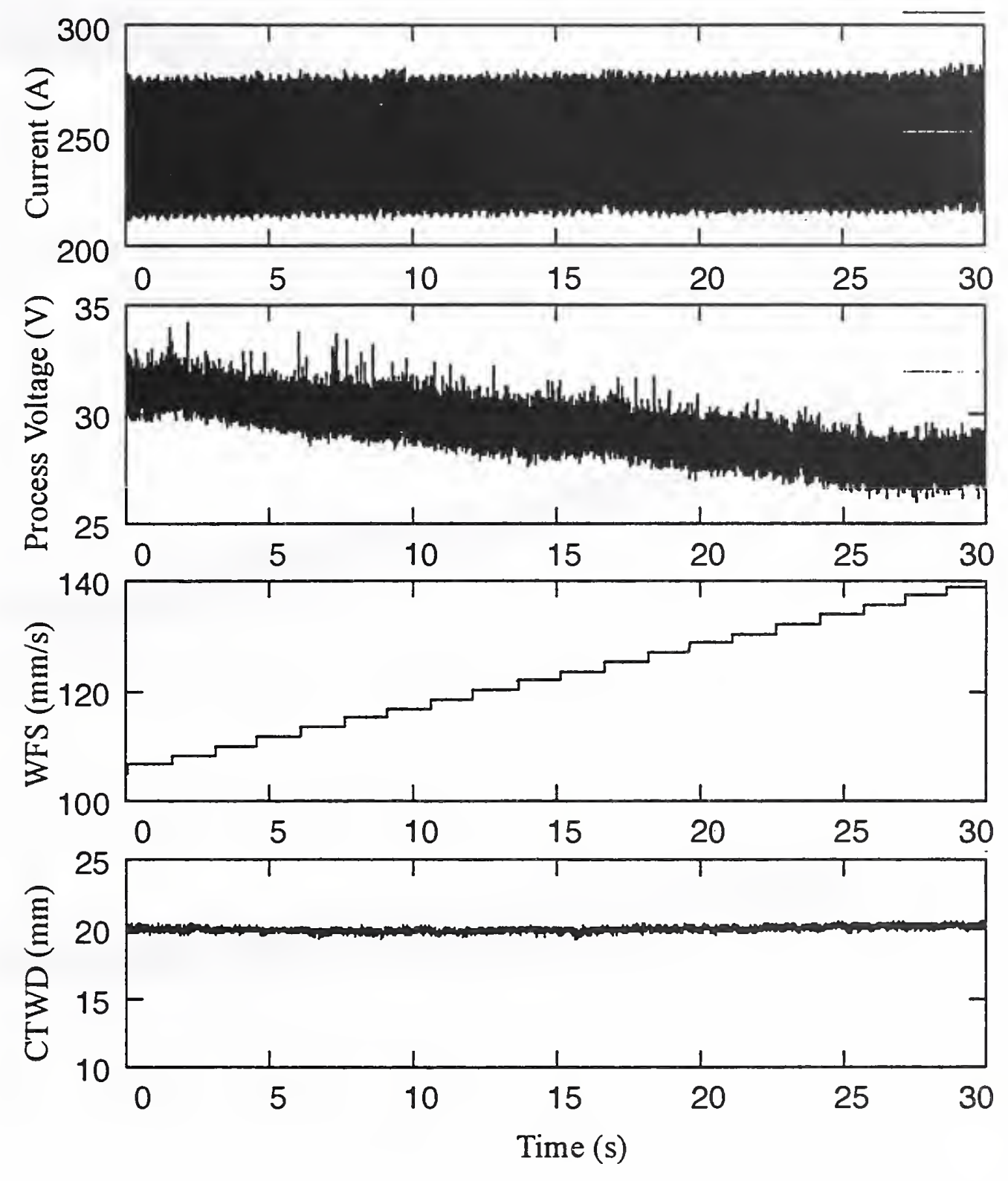

Figure 35. Time traces for the process inputs during welding. The current is modulated with a triangular waveform at $25 \mathrm{~Hz}$ about a mean level. The WFS is incremented as the weld progresses while the CTWD is constant. 



Figure 36. Time traces for the process-output parameters. The electrode extension increases and the arc length decreases in response to increases in WFS. The mean and modulation amplitude of the arc-light-detector voltage decrease with arc length. 


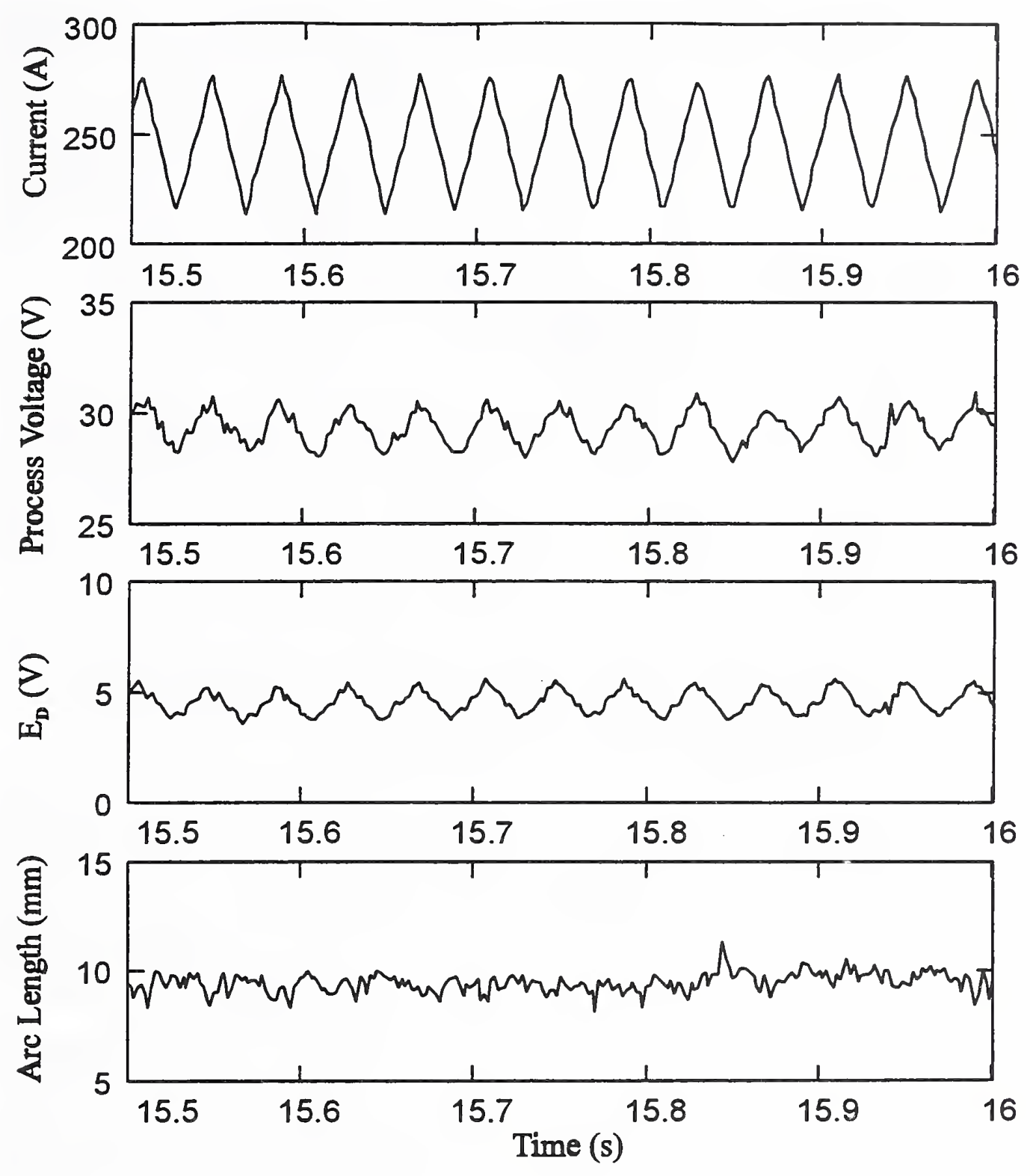

Figure 37. A section of the waveforms shown in figures 35 and 36. The WFS is $123 \mathrm{~mm} / \mathrm{s}$, the CTWD is $20 \mathrm{~mm}$, and the arc length is $9 \mathrm{~mm}$. The current trace shows the triangular waveform of the input reference; the process voltage and detector voltage show similar behavior. 


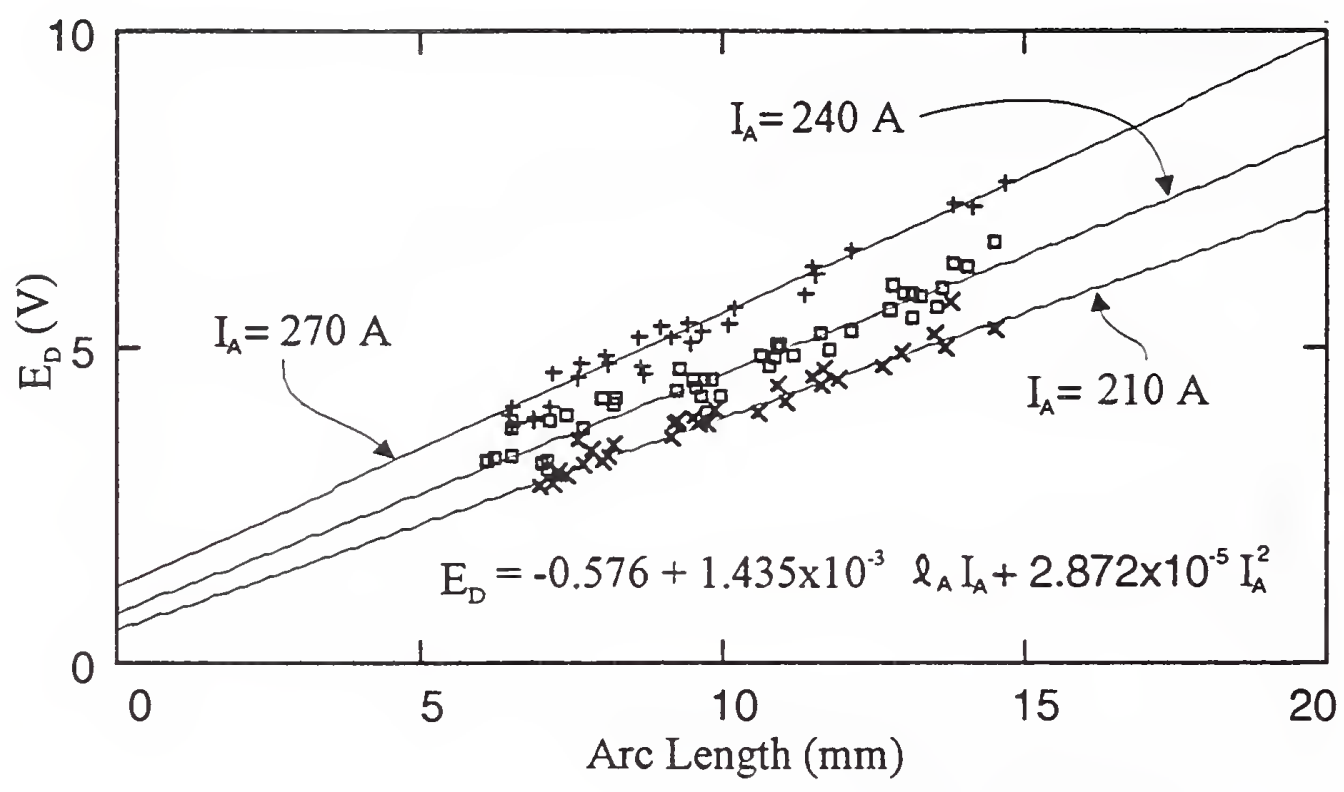

Figure 38. Detector voltage versus arc length for several values of current. The detector voltage (arc-light intensity) increases linearly with arc length, and the slope increases with increasing current.

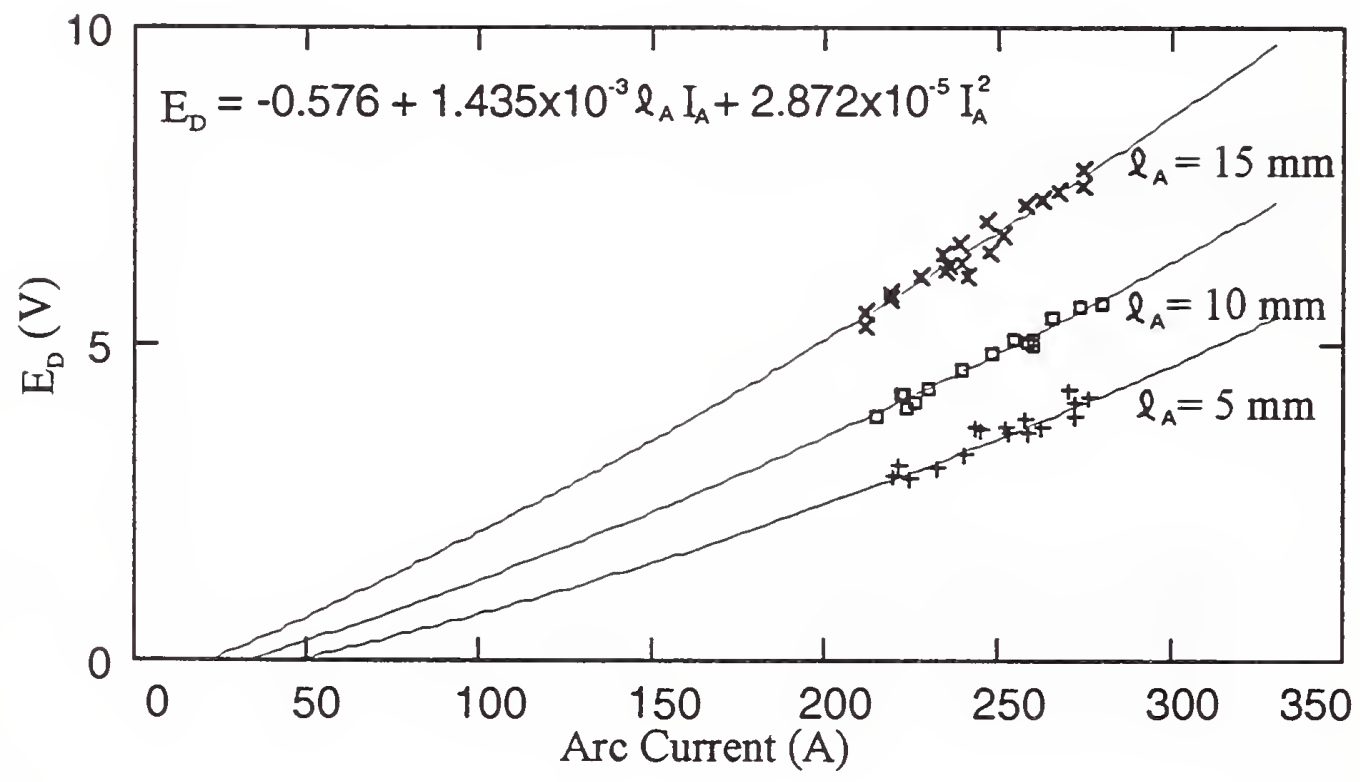

Figure 39. Arc current versus detector voltage for several arc lengths.

The detector voltage increases with current at a fixed arc length. 
Extrapolation to zero arc length shows a positive detector voltage. This offset predicts that there is some arc light even when the arc length is 0 . Figure 39 shows a plot of detector voltage versus arc current for several arc lengths. The plot predicts that the detector voltage drops to 0 while the arc current is positive. The curves also show the nonlinear increase in detector voltage with current at a fixed arc length. The curves of constant arc length, when plotted in the range 200 to $300 \mathrm{~A}$, appear nearly linear. This explains why inspection of the curves over the limited current range led to the prediction that detector voltage is a linear function of current, as described in eq (34).

In an attempt to explain why the detector voltage extrapolated to a nonzero value at zero arc length and zero current, another weld was made. Here, the current was constant and the arc length was $6 \mathrm{~mm}$. To estimate the magnitude of light intensity from the electrode and weld pool, the welding current and wire feed were abruptly stopped while data collection continued. Figure 40 shows the resulting current and detector-voltage time traces. Before arc extinction, the average current was $258 \mathrm{~A}$, and the average detector voltage was $4.8 \mathrm{~V}$. Within $2 \mathrm{~ms}$ after the welding stopped, the current dropped to 0 and the detector voltage dropped to $0.26 \mathrm{~V}$, which is attributed to weld-pool and electrode-tip radiation. The detector voltage continued to decrease as the pool and electrode cooled. The ratio of detector-voltage values before and after arc extinction was $0.26 \mathrm{~V} / 4.8 \mathrm{~V}$ or 0.054 . Thus, the weld-pool and electrode-tip light intensity are about 5 percent of the total light intensity and cannot fully account for the observed detector-voltage offset of 0.5 to $1.25 \mathrm{~V}$.

The remainder of the detector-voltage offset can be explained by the way arc length is defined. The arc length measured from the video images was defined as the distance between the end of the electrode and the top of the weld pool. There are two possible sources of error in the definition: (1) In the range of current used here, the weld-pool surface can be depressed by the pressure exerted from the arc, causing the arc to extend below the top of the workpiece surface. (2) Some electrons condense on the solid electrode, causing the arc to extend above the end of the electrode. In either case, the actual arc length may be longer than that measured. A difference of 1 to $2 \mathrm{~mm}$ in arc length can account for the offset in detector voltage.

A convenient way to display and compare the results from welding is to plot the measured arc length against the estimated arc length (see fig. 41). The correlation parameter $R_{c}$ is 0.93 , indicating a good fit between the measured and estimated arc length. Figure 41 also shows a histogram of the difference between the actual and estimated arc length (the error) for each point in the data set. The error has a normal distribution with a standard deviation $\sigma_{\mathrm{c}}$ of $0.9 \mathrm{~mm}$.

\subsubsection{Source/Detector Angle}

The changes in detector voltage, correlation parameter, and error standard deviation as a function of the source/detector angle $\theta_{S}$ are shown in figure 42 . The results are from welding using the BBP optical filter, $98 \% \mathrm{Ar}+2 \% \mathrm{O}_{2}$ shielding gas, $\theta_{D}=0^{\circ}$, and $R_{D}=400 \mathrm{~mm}$. In figure $42 \mathrm{a}$, the detector voltage $E_{D}$ was calculated from the set of coefficients found for eq (42) at each value of $\theta_{S}$. The current was $250 \mathrm{~A}$ and the arc length was $6 \mathrm{~mm}$. As $\theta_{S}$ increased, the detector voltage decreased for the same arc length and current; the decrease in $E_{D}$ is attributed to a 




Figure 40. Current and arc-light-detector voltage response to an abrupt stop in welding at $t=0 \mathrm{~ms}$. The detector voltage after arc extinction $(0.26 \mathrm{~V})$ is attributed to weld-pool and electrode-tip radiation and is approximately 5 percent of the radiation when the arc is on. 


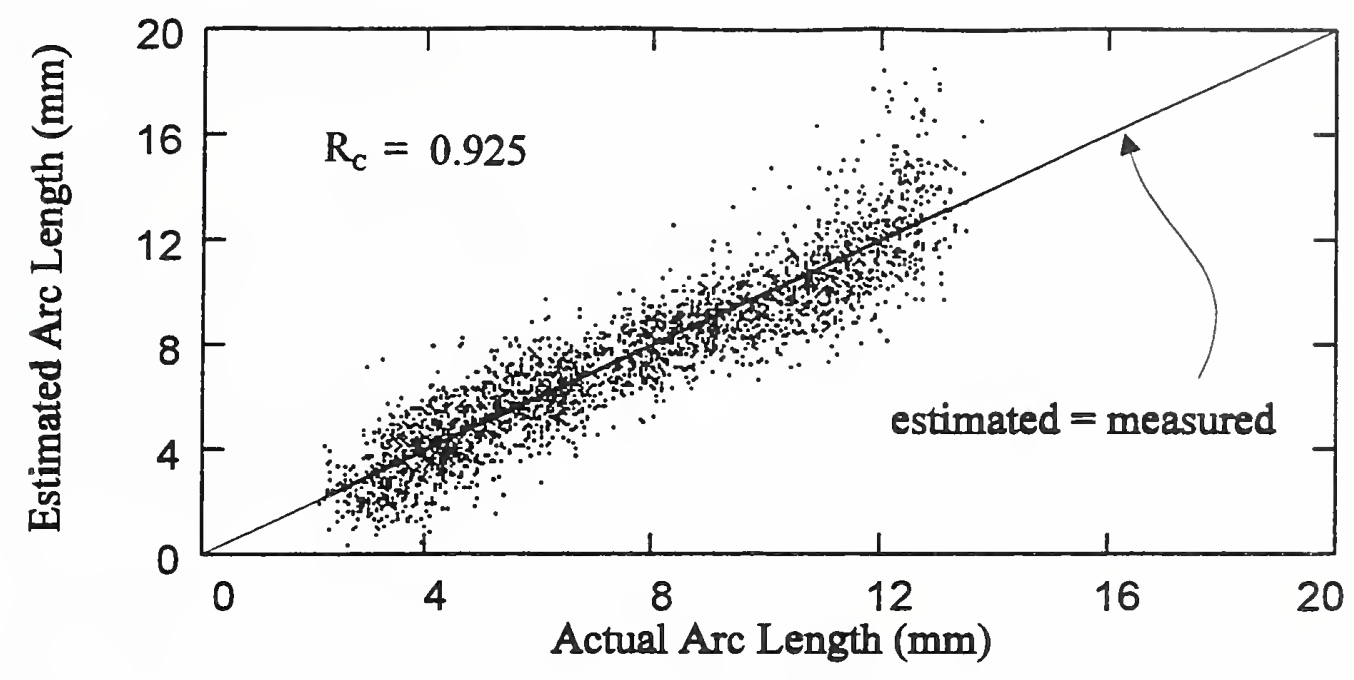

a.)

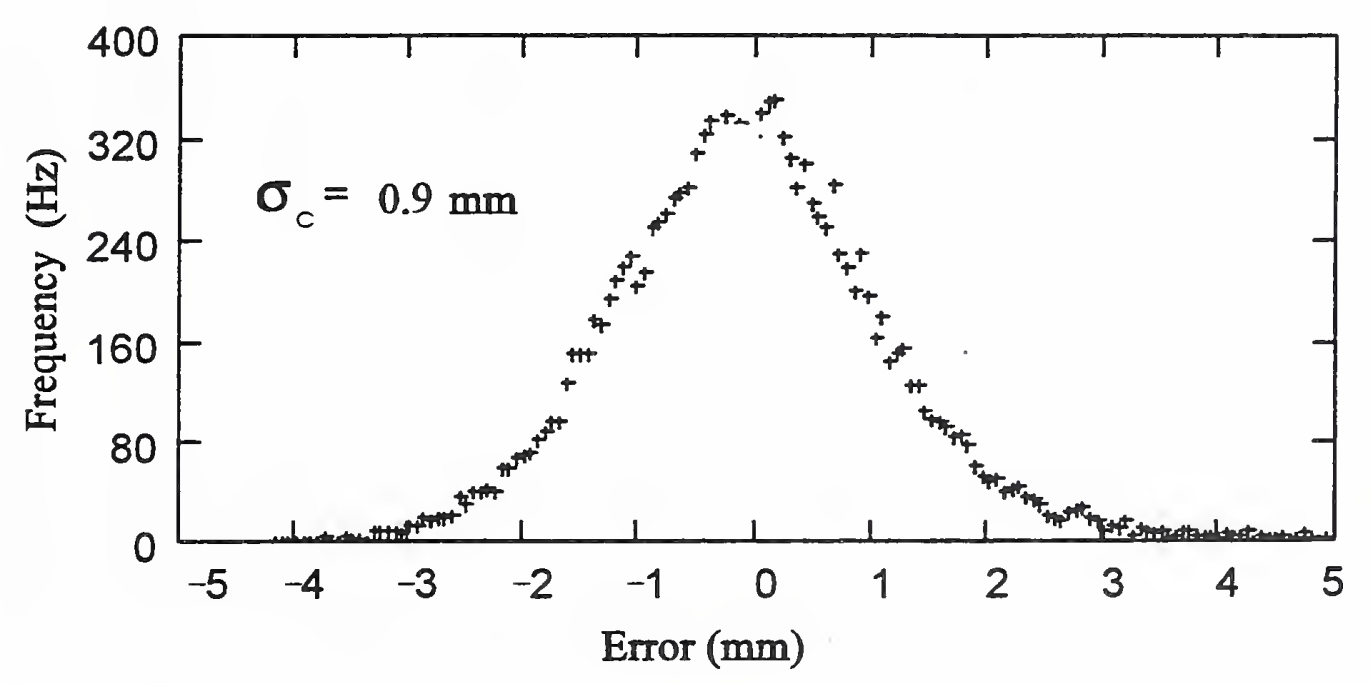

b.)

Figure 41. Example of correlation analysis results: (a) measured arc length versus estimated arc length illustrating data scatter and the correlation parameter $R_{c}$; (b) histogram of the error (difference) between the actual and estimated arc length for each point in the data set showing a normal distribution with a standard deviation $\sigma_{c}$. 

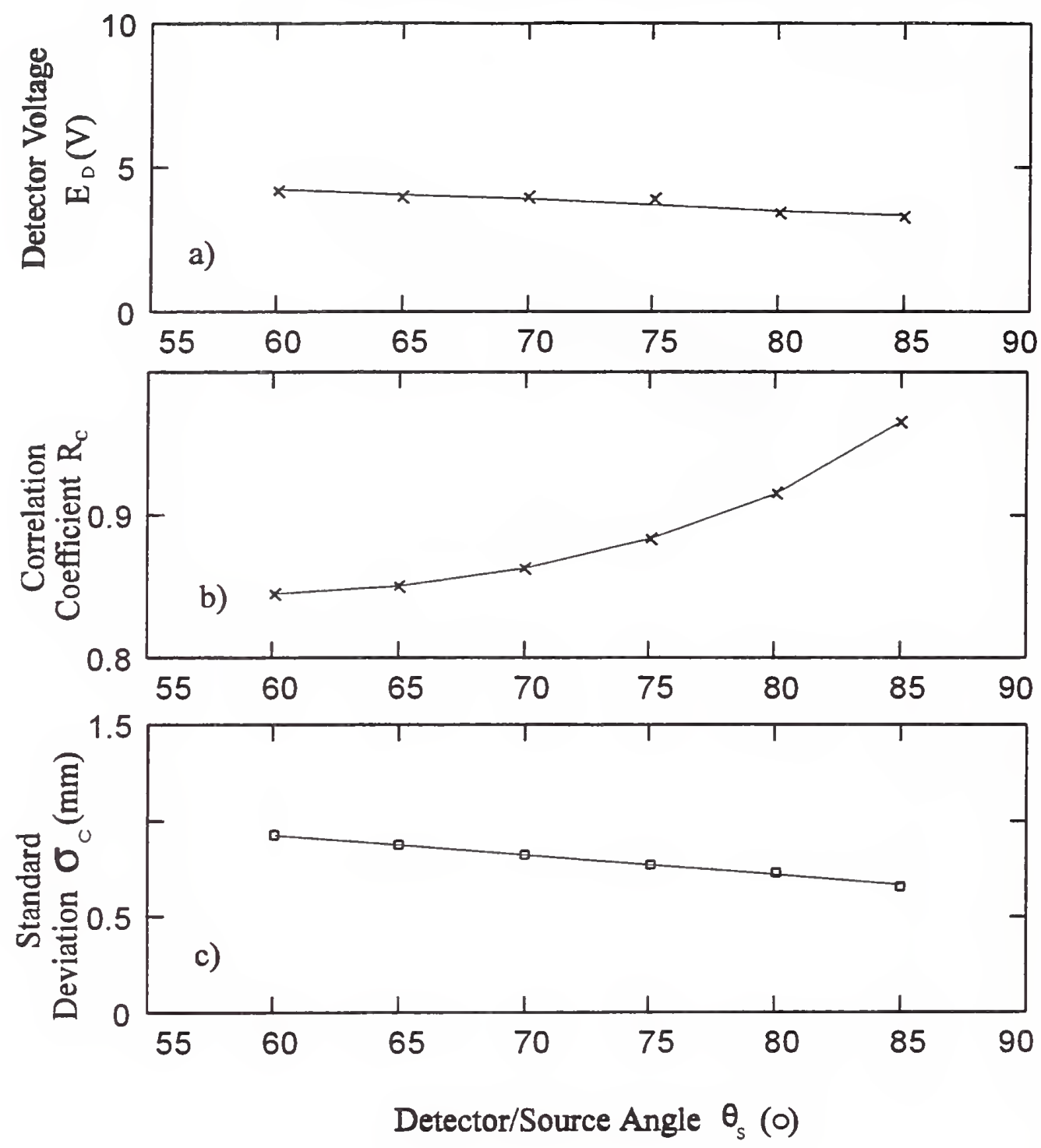

Figure 42. Detector voltage, correlation parameter, and error standard deviation as a function of the source/detector angle $\theta_{S}$ from welding using the BBP optical filter, $98 \% \mathrm{Ar}+2 \% \mathrm{O}_{2}$ shielding gas, $\theta_{D}=0^{\circ}$, and $R_{D}=400 \mathrm{~mm}$. Results indicate that $\theta_{S}$ should be as close to $90^{\circ}$ as possible to obtain the best estimate of arc length. 
decrease in blackbody radiation from the pool and a decrease in the arc light reflected by the pool and workpiece.

Figures $42 \mathrm{~b}$ and $42 \mathrm{c}$ show that as $\theta_{S}$ increases, the correlation parameter $R_{c}$ increases and the error standard deviation $\sigma_{c}$ decreases. The decrease in $\sigma_{c}$ and increase in $R_{c}$ with $\theta_{S}$ indicate that better arc-length estimation is obtained for larger values of $\theta_{S}$. All other combinations of optical filter and shielding gas showed similar trends in $E_{D}, R_{c}$, and $\sigma_{c}$ as a function of $\theta_{S}$. We conclude that $\theta_{S}$ should be as close to $90^{\circ}$ as possible to obtain the best estimate of arc length.

\subsubsection{Detector Orientation}

The changes in detector voltage, correlation parameter, and error standard deviation as a function of the detector orientation angle $\theta_{D}$ are shown in figure 43. The results are from welding using the BBP optical filter with $98 \% \mathrm{Ar}+2 \% \mathrm{O}_{2}$ shielding gas, $\theta_{S}=85^{\circ}$, and $R_{D}=400 \mathrm{~mm}$. In Figure $43 \mathrm{a}$, the detector voltage $E_{D}$ was calculated at each value of $\theta_{D}$ in the same manner as in the previous section. The detector voltage decreased for the same arc length and current as $\theta_{D}$ increased and followed the cosine dependence predicted from eq (45).

Figures $43 \mathrm{~b}$ and $43 \mathrm{c}$ show that as $\theta_{D}$ increased, the correlation parameter $R_{c}$ decreased and the error standard deviation $\sigma_{c}$ increased. The increase in $\sigma_{c}$ and decrease in $R_{c}$ with $\theta_{D}$ indicate that better arc-length estimation was obtained for small values of $\theta_{S}$; however, some misalignment of the detector could be tolerated. All other combinations of optical filter and shielding gas showed similar trends in $E_{D}, R_{c}$, and $\sigma_{c}$ as a function of $\theta_{D}$. Thus, the detector should be in direct line of site of the arc or $\theta_{D}$ should be as close to $0^{\circ}$ as possible to obtain the best estimate of arc length.

\subsubsection{Detector Distance}

Changes in detector voltage, correlation parameter, and error standard deviation as a function of the detector distance $R_{D}$ are shown in figure 44. The results are from welding using the BBP optical filter, $98 \% \mathrm{Ar}+2 \% \mathrm{O}_{2}$ shielding gas, $\theta_{S}=85^{\circ}$, and $\theta_{D}=0^{\circ}$. In Figure $44 \mathrm{a}$, the detector voltage $E_{D}$ was calculated at each value of $R_{D}$ in the same manner as in the previous section. The detector voltage decreased for the same arc length and current while $R_{D}$ increased and followed the $1 / R_{D}{ }^{2}$ dependence predicted from eq (45).

Figures $44 \mathrm{~b}$ and $44 \mathrm{c}$ show that as $R_{D}$ increased, the correlation parameter $R_{c}$ decreased, and the error standard deviation $\sigma_{c}$ increased. The increase in $\sigma_{c}$ and decrease in $R_{c}$ with $R_{D}$ are attributed to the decrease in arc-length resolution caused by a decrease in the range of detector output voltage and the fixed ADC resolution. The data points indicated by $x$ at $600 \mathrm{~mm}$ in figures $44 \mathrm{a}, \mathrm{b}$, and $\mathrm{c}$ result from increasing the detector amplifier gain by 50 percent. Increasing the detector gain increased the detector voltage range and, therefore, the arc-length resolution. The increase in arclength resolution restored $R_{c}$ and $\sigma_{c}$ to values obtained with a smaller $R_{D}$.

These results indicate that the detector can operate over a variety of distances provided its output-voltage range is approximately equal to the ADC range. The detector output voltage can be adjusted by the detector amplifier gain and ND filtering. The results of the experiments to determine the optimum source/detector angle, detector orientation angle, and source/detector distance are summarized in table 1. 

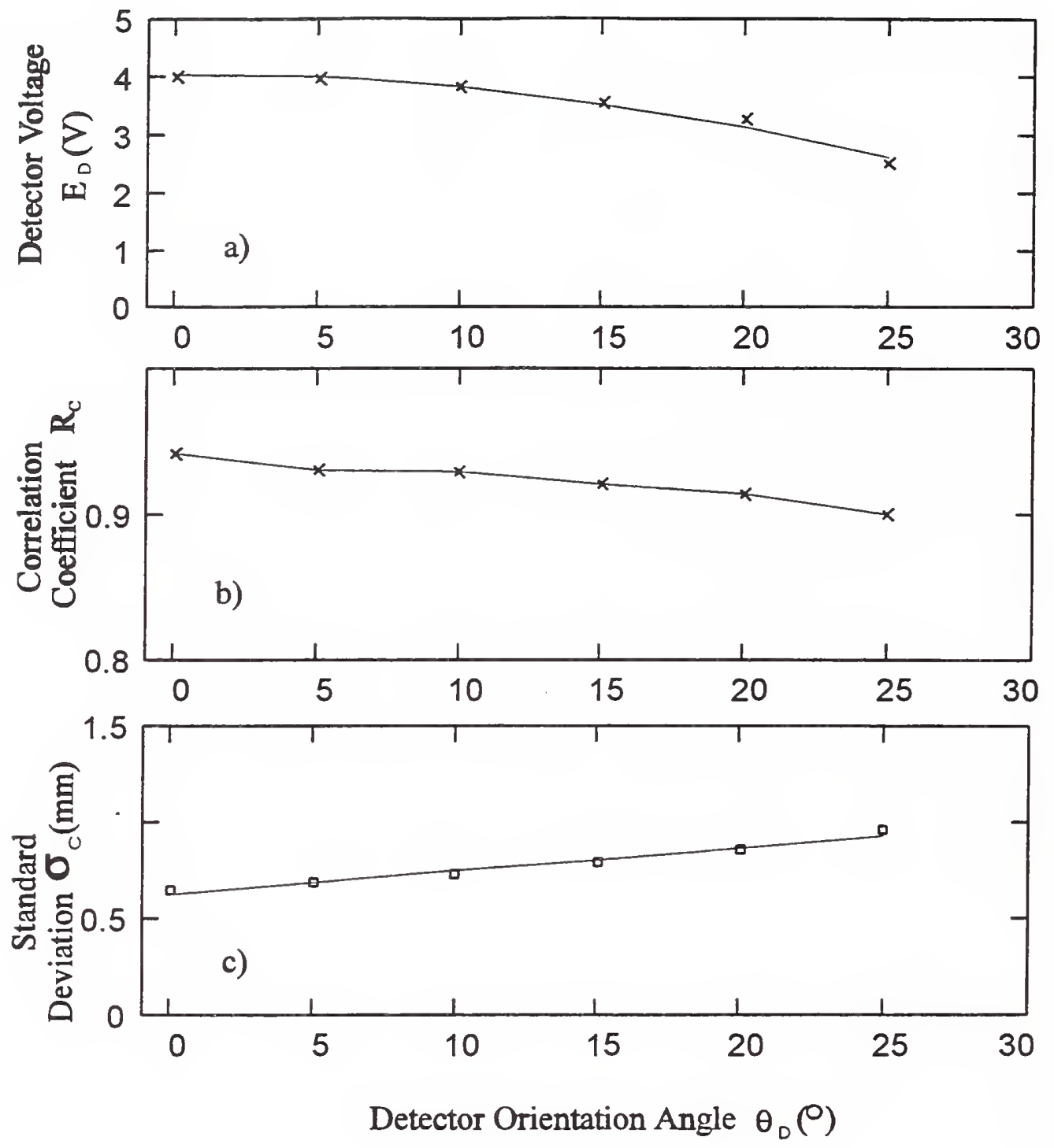

Figure 43. Detector voltage, correlation parameter, and standard deviation of the error as a function of the detector orientation angle $\theta_{D}$ from welding using the BBP optical filter, $98 \% \mathrm{Ar}+2 \% \mathrm{O}_{2}$ shielding gas, $\theta_{S}$ $=85^{\circ}$, and $R_{D}=400 \mathrm{~mm}$. Results indicate that $\theta_{D}$ should be as close to $0^{\circ}$ as possible to obtain the best estimate of arc length. 


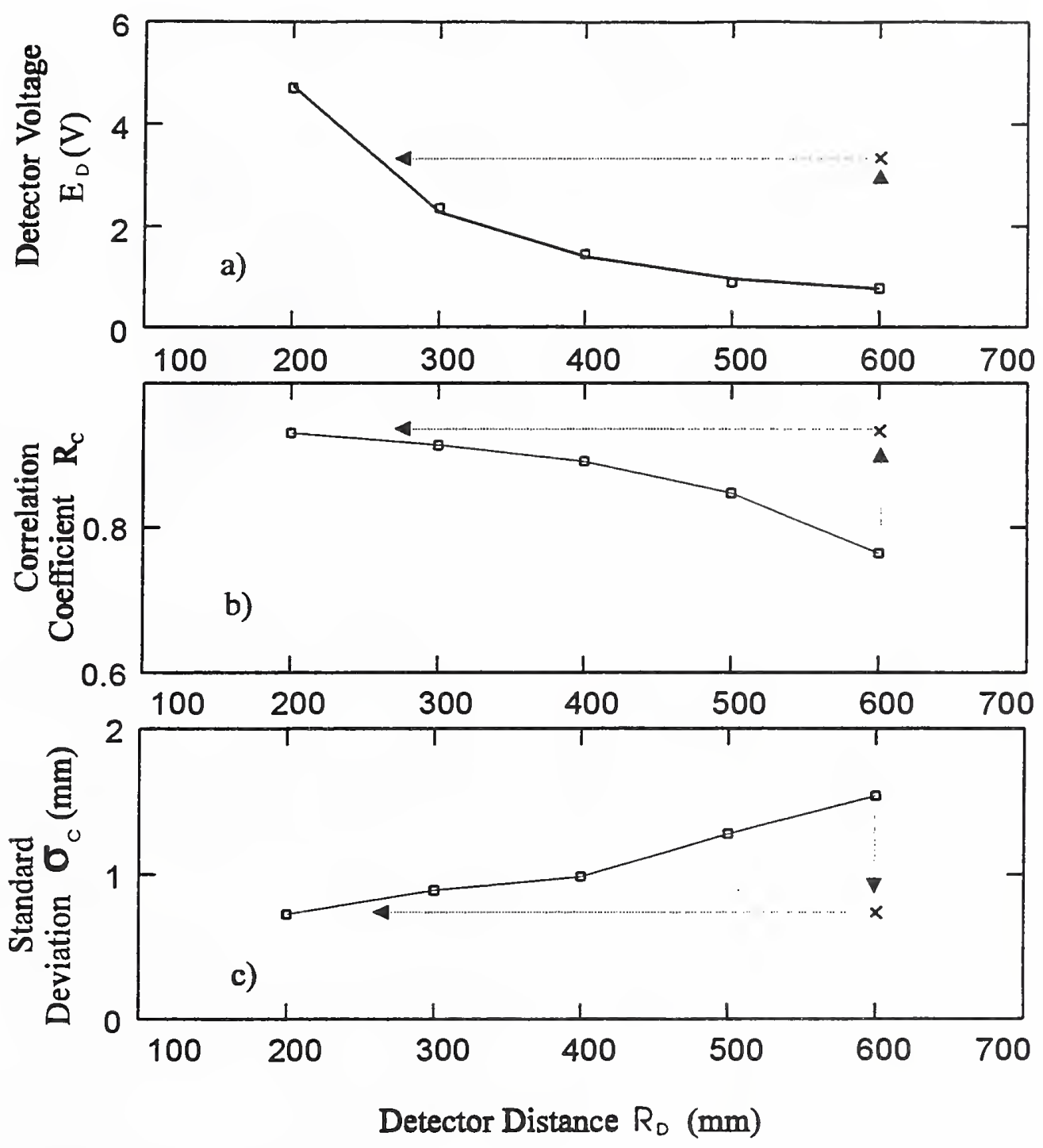

Figure 44. Detector voltage, correlation parameter and error standard deviation as a function of the detector distance $R_{D}$ from welding using the BBP optical filter, $98 \% \mathrm{Ar}+2 \% \mathrm{O}_{2}$ shielding gas, $\theta_{S}=85^{\circ}$, and $\theta_{D}=0^{\circ}$. Results indicate that the detector can operate over a range of distance if its output-voltage range is approximately equal to the ADC range. 
Table 1. Optimum geometric configuration relative to the arc for arc-length estimation.

\begin{tabular}{ccc}
\hline $\begin{array}{c}\text { Source/Detector } \\
\text { Angle } \theta_{S}\left(^{\circ}\right)\end{array}$ & $\begin{array}{c}\text { Detector Orientation } \\
\text { Angle } \theta_{D}\left(^{\circ}\right)\end{array}$ & $\begin{array}{c}\text { Detector Distance, } \\
R_{D}(\mathrm{~mm})\end{array}$ \\
\hline 85 & 0 & $15<R_{D}<600^{*}$ \\
\hline
\end{tabular}

${ }^{*} R_{D}$ can assume any distance in the noted range provided that the detector gain or ND filtering is adjusted to produce a detector-voltage output range equivalent to the $\mathrm{ADC}$ range.

\subsubsection{Optical Filters and Shielding Gas}

A final set of welds was made to determine the effect of optical filters and shielding gas on arc-length estimation by using the detector angles shown in table 1 . The detector distance $R_{D}$ was set at $400 \mathrm{~mm}$ simply because that distance best accommodated the workpiece fixture and detector arm.

The results for each combination of optical filter and shielding gas are presented as plots of measured arc length versus estimated arc length and the error histograms in figures 45 through 54 . The results are given in the order of improved performance of arc-length estimation, grouped by optical filter. The data scatter was greatest at the extreme arc lengths, lengths that are outside those used in most situations. This data scatter was caused by arc instabilities, and an improvement in arc-length estimation could be obtained by eliminating data outside the practical arc lengths. The values obtained for the coefficients $\mathrm{C}_{0}, \mathrm{C}_{1}$, and $\mathrm{C}_{2}$ and the corresponding values of $R_{c}$ and $\sigma_{c}$ for each combination of optical filter and shielding gas are provided in table 2.

The poorest results were obtained from the $467-\mathrm{nm}$ filter, which is sensitive to radiation associated with continuum production processes (photorecombination and bremsstrahlung). While the trend of increasing continuum radiation with both arc length and current was observed, the relatively large data scatter (see figs. 45 and 46) shows that the continuum radiation provided less of an indication of arc length than the other spectral locations. The results from the 430 -nm filter, sensitive primarily to Fe lines, also showed considerable data scatter (see figs. 47 and 48). As will be shown in next section, the greatest variation in arc-light intensity associated with droplet detachment occurred with the 430-nm filter. Thus, the scatter in data is attributed to the noise associated with droplet detachment.

In the case of the 694-nm filter, which is sensitive to Ar radiation, the performance for both shielding gases was nearly identical (figs. 49 and 50). Since both gases are Ar based, the results indicate that the variation in arc-light intensity from Ar as a function of arc length and current was similar for both gases. The results of the ND filter are shown in figures 51 and 52. The ND filter integrates radiation over all 

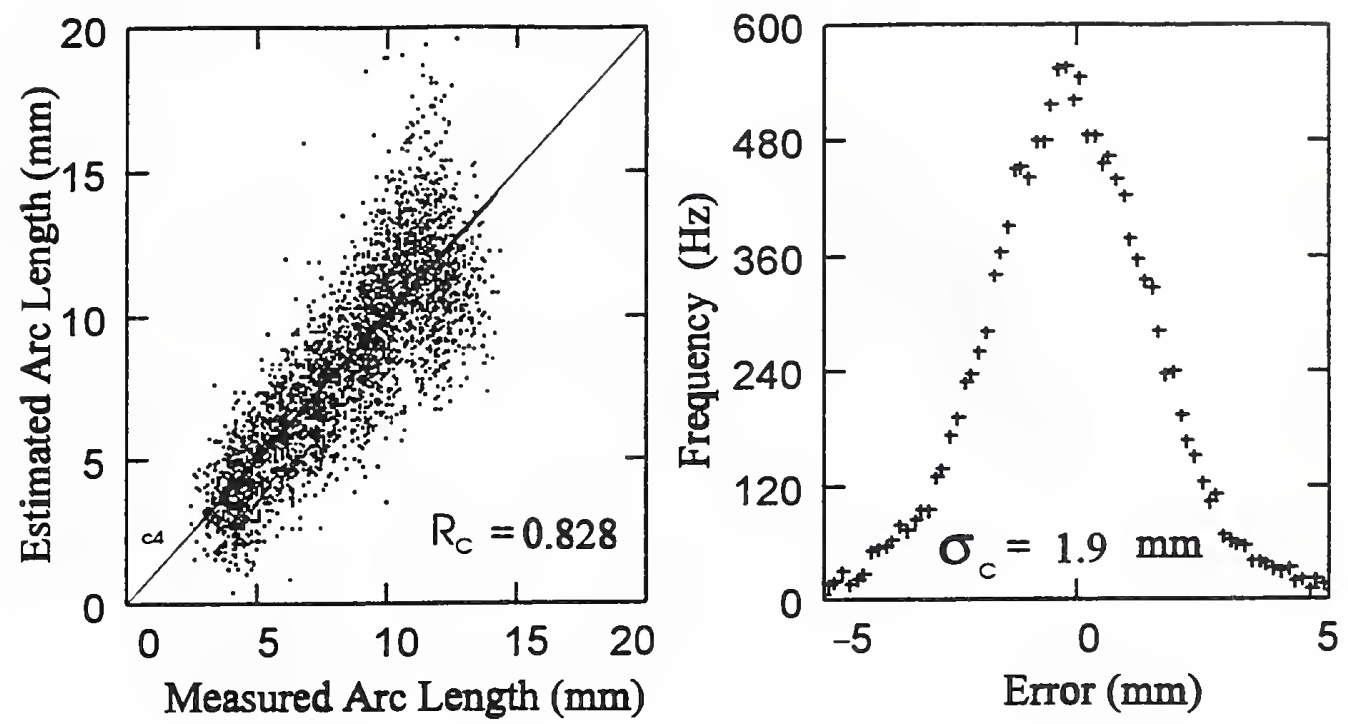

Figure 45. Estimated versus measured arc length and error histogram resulting from using the $467-\mathrm{nm}$ optical filter and $95 \% \mathrm{Ar}+5 \% \mathrm{CO}_{2}$ shielding gas.
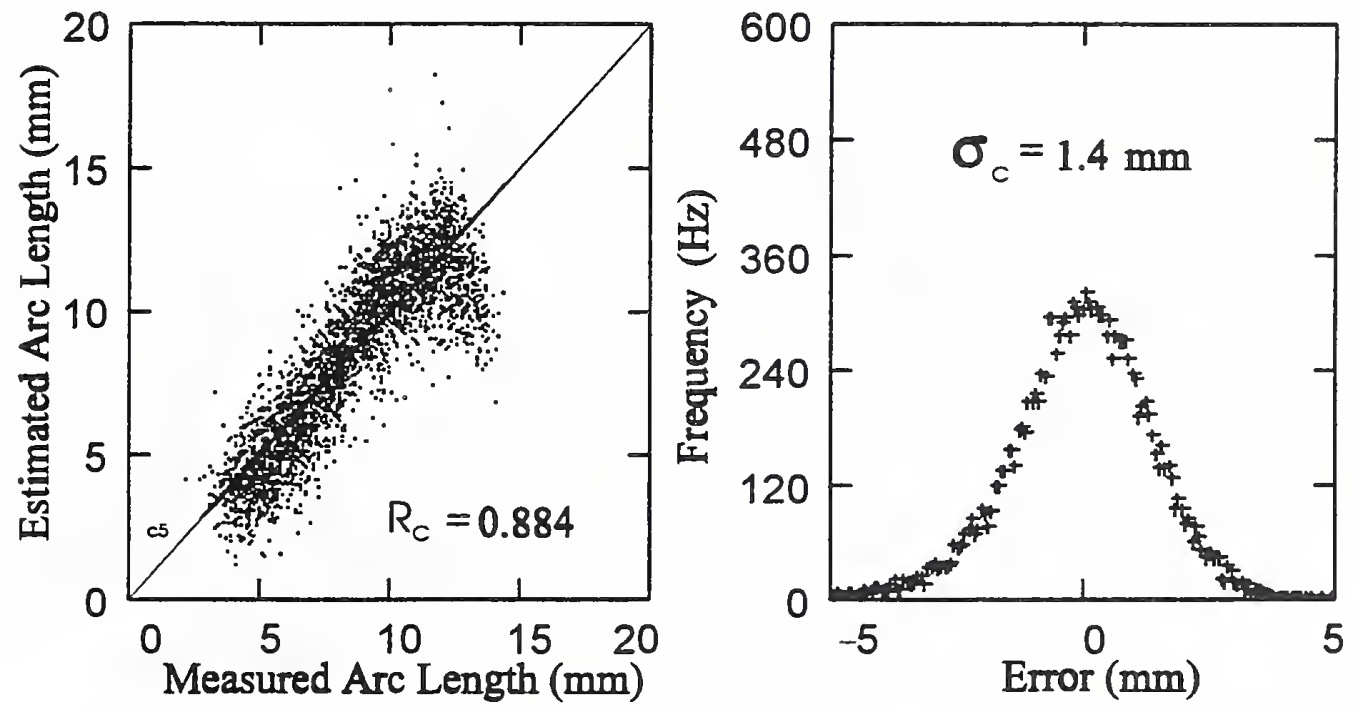

Figure 46. Estimated versus measured arc length and error histogram resulting from using the $467-\mathrm{nm}$ optical filter and $98 \% \mathrm{Ar}+2 \% \mathrm{O}_{2}$ shielding gas. 

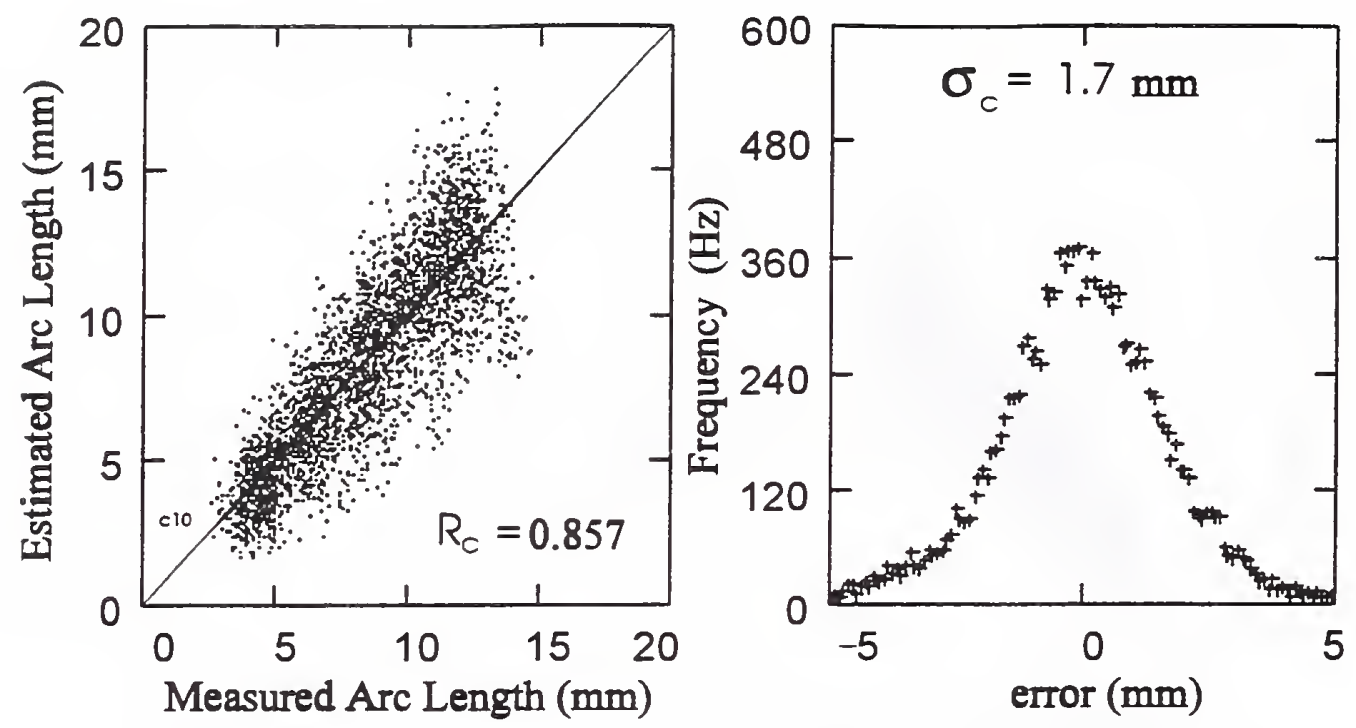

Figure 47. Estimated versus measured arc length and error histogram resulting from using the $430-\mathrm{nm}$ optical filter and $95 \% \mathrm{Ar}+5 \% \mathrm{CO}_{2}$ shielding gas.
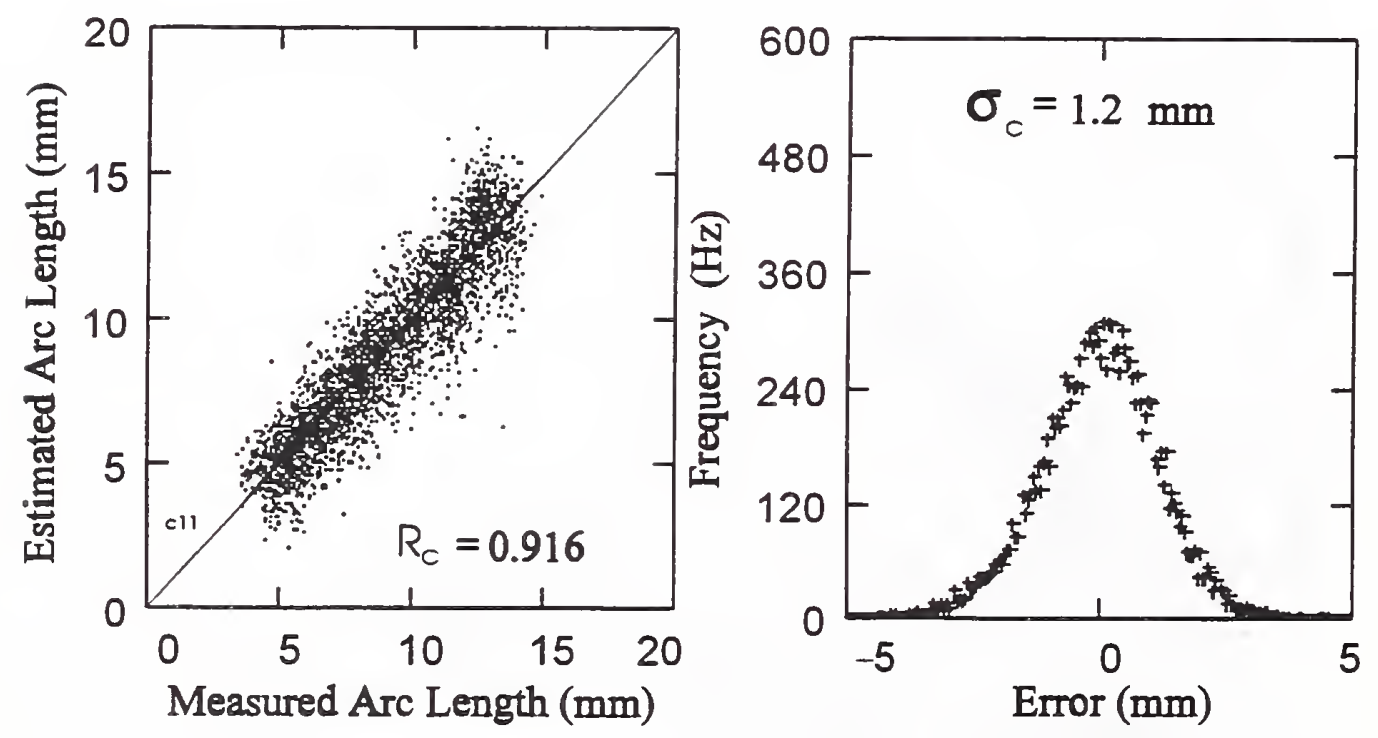

Figure 48. Estimated versus measured arc length and error histogram resulting from using the $430-\mathrm{nm}$ optical filter and $98 \% \mathrm{Ar}+2 \% \mathrm{O}_{2}$ shielding gas. 

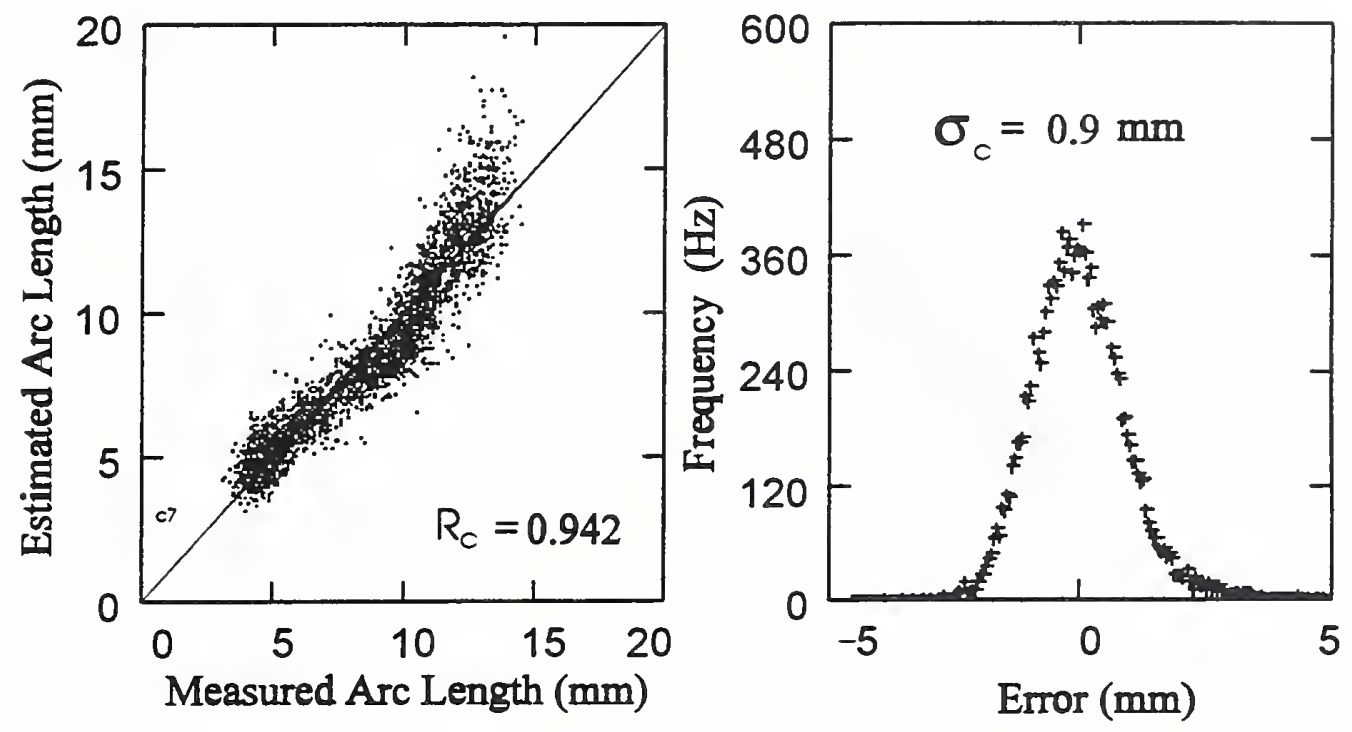

Figure 49. Estimated versus measured arc length and error histogram resulting from using the $694-\mathrm{nm}$ optical filter and $95 \% \mathrm{Ar}+5 \% \mathrm{CO}_{2}$ shielding gas.
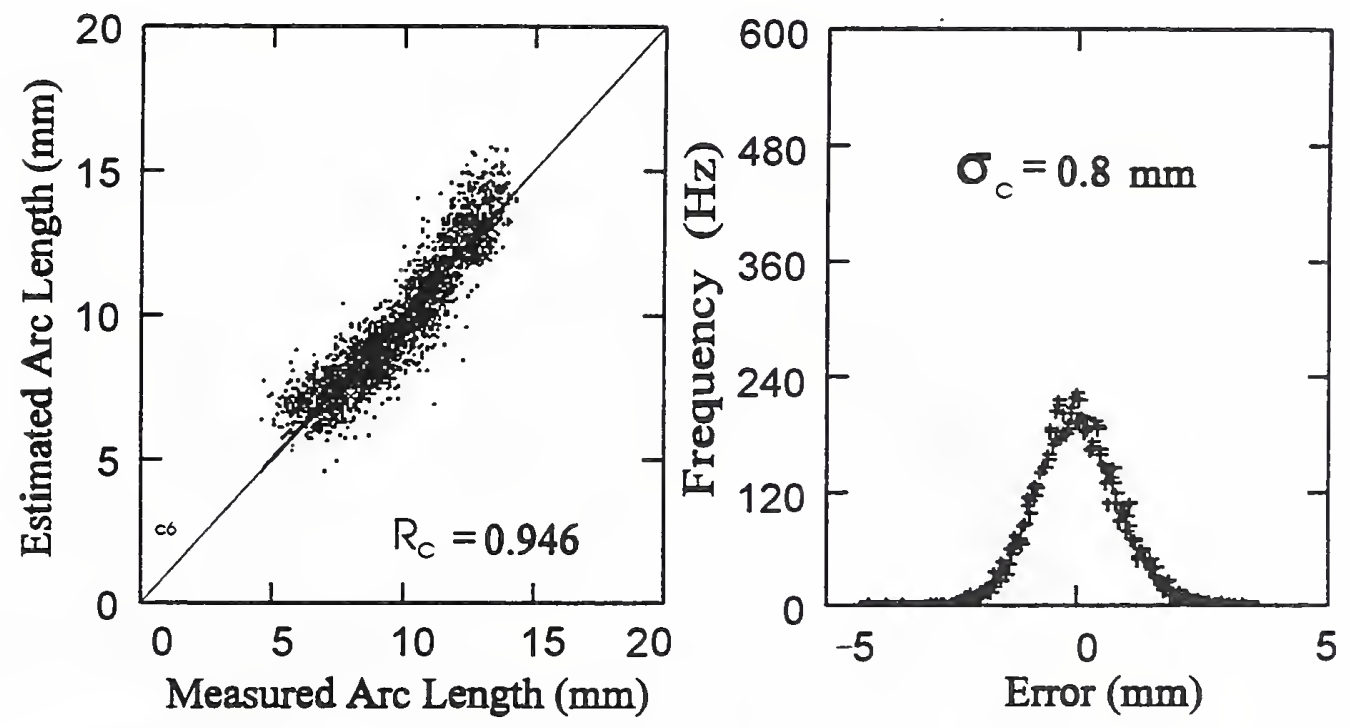

Figure 50. Estimated versus measured arc length and error histogram resulting from using the 694-nm optical filter and $98 \% \mathrm{Ar}+2 \% \mathrm{O}_{2}$ shielding gas. 

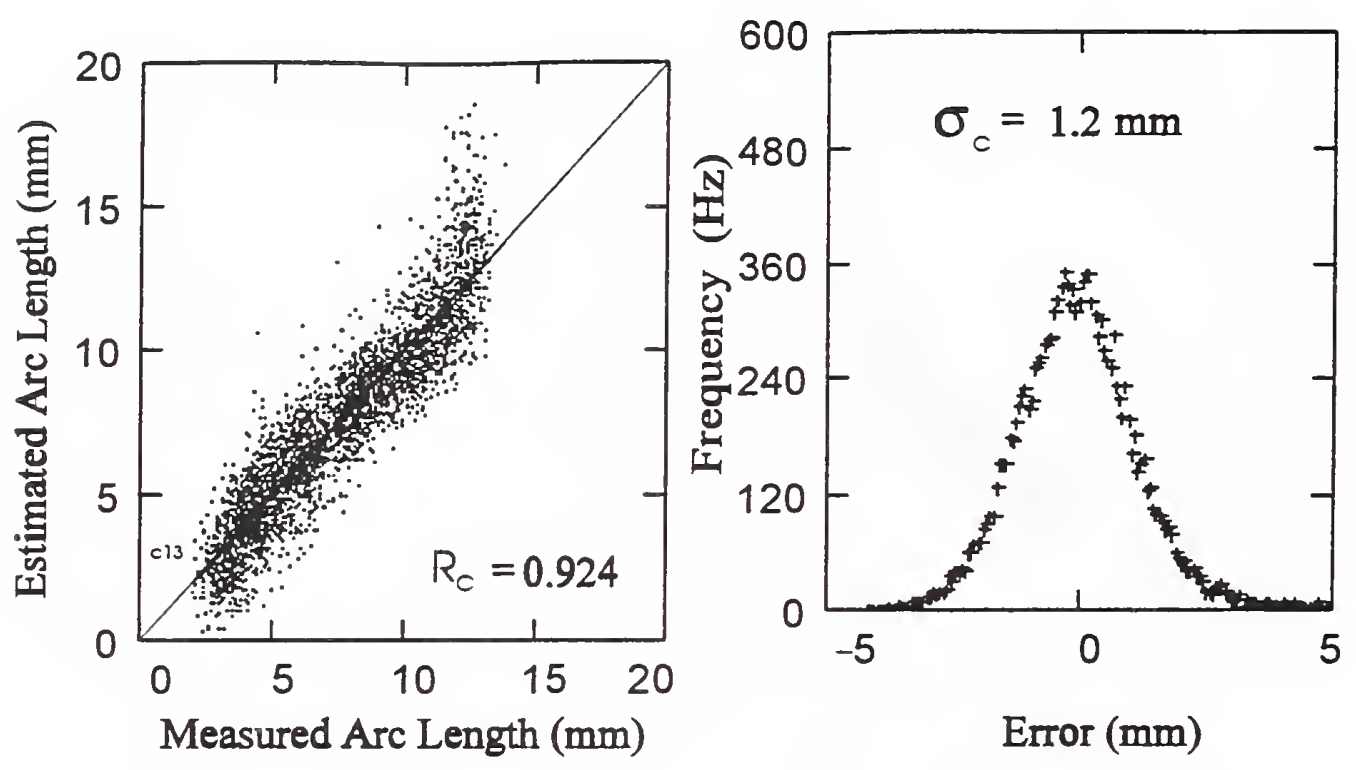

Figure 51. Estimated versus measured arc length and error histogram resulting from using the ND optical filter and $95 \% \mathrm{Ar}+5 \% \mathrm{CO}_{2}$ shielding gas.
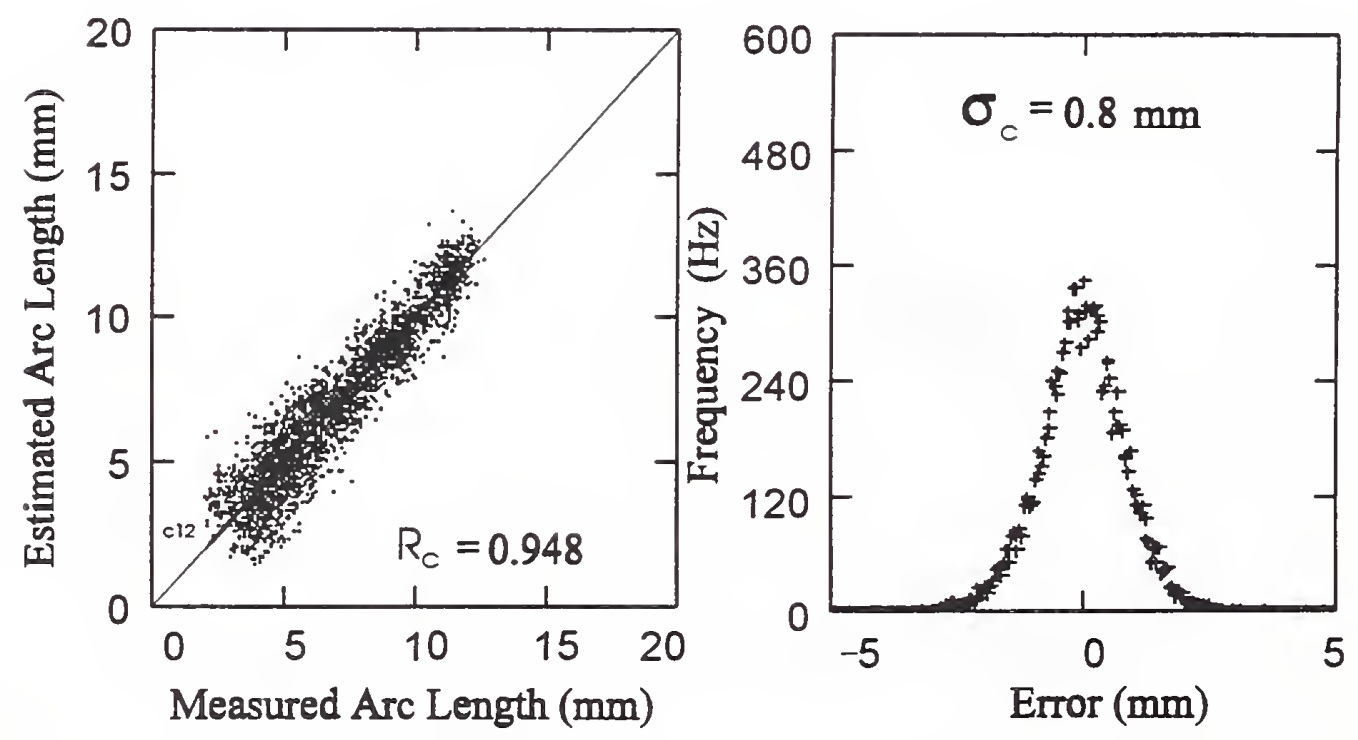

Figure 52. Estimated versus measured arc length and error histogram resulting from using the $\mathrm{ND}$ optical filter and $98 \% \mathrm{Ar}+2 \% \mathrm{O}_{2}$ shielding gas. 



Figure 53. Estimated versus measured arc length and error histogram resulting from using the $\mathrm{BBP}$ optical filter and $95 \% \mathrm{Ar}+5 \% \mathrm{CO}_{2}$ shielding gas.
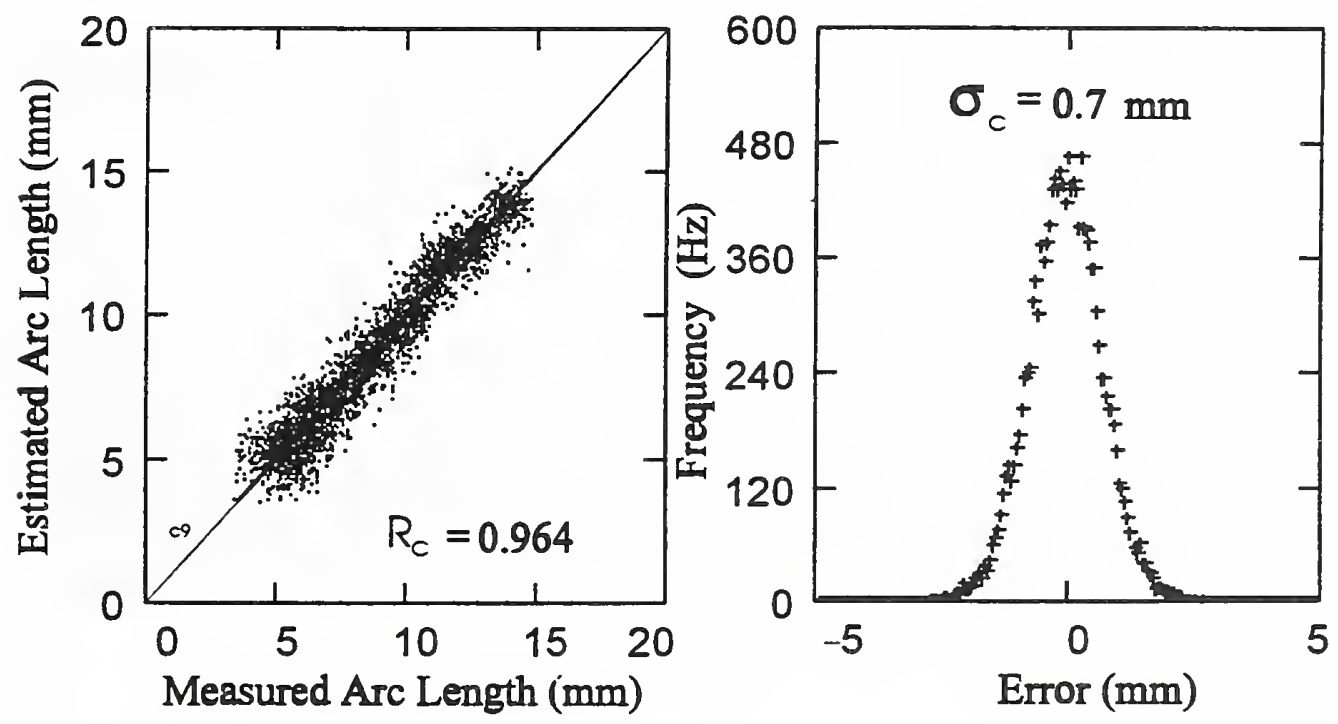

Figure 54. Estimated versus measured arc length and error histogram resulting from using the BBP optical filter and $98 \% \mathrm{Ar}+2 \% \mathrm{O}_{2}$ shielding gas. 
Table 2. Summary of coefficients for the arc-length-sensor model [eq (43)], correlation parameter, and standard deviation for each combination of shielding gas and optical filter using the detector location and orientation shown in table 1.

\begin{tabular}{llccccc}
\hline $\begin{array}{l}\text { Optical } \\
\text { Filter }\end{array}$ & \multicolumn{1}{c}{ Shielding Gas } & $\mathrm{C}_{0}(\mathrm{~V})$ & $\begin{array}{c}\mathrm{C}_{1} \times 10^{-3} \\
{[\mathrm{~V} /(\mathrm{mm} \cdot \mathrm{A})]}\end{array}$ & $\begin{array}{c}\mathrm{C}_{2} \times 10^{-6} \\
\left(\mathrm{~V} / \mathrm{A}^{2}\right)\end{array}$ & $R_{c}$ & $\begin{array}{c}\sigma_{c} \\
(\mathrm{~mm})\end{array}$ \\
\hline $467 \mathrm{~nm}$ & $98 \% \mathrm{Ar}+2 \% \mathrm{O}_{2}$ & 0.223 & 0.809 & 22.7 & 0.884 & 1.4 \\
$467 \mathrm{~nm}$ & $95 \% \mathrm{Ar}+5 \% \mathrm{CO}_{2}$ & 0.189 & 0.757 & 23.6 & 0.828 & 1.9 \\
$430 \mathrm{~nm}$ & $98 \% \mathrm{Ar}+2 \% \mathrm{O}_{2}$ & -0.122 & 1.27 & 32.2 & 0.916 & 1.2 \\
$430 \mathrm{~nm}$ & $95 \% \mathrm{Ar}+5 \% \mathrm{CO}_{2}$ & -0.182 & 1.19 & 36.3 & 0.857 & 1.7 \\
$694 \mathrm{~nm}$ & $98 \% \mathrm{Ar}+2 \% \mathrm{O}_{2}$ & -0.864 & 1.50 & 23.5 & 0.946 & 0.8 \\
$694 \mathrm{~nm}$ & $95 \% \mathrm{Ar}+5 \% \mathrm{CO}_{2}$ & -0.513 & 0.971 & 22.5 & 0.942 & 0.9 \\
$\mathrm{ND}$ & $98 \% \mathrm{Ar}+2 \% \mathrm{O}_{2}$ & -0.096 & 1.35 & 30.3 & 0.948 & 0.8 \\
$\mathrm{ND}$ & $95 \% \mathrm{Ar}+5 \% \mathrm{CO}_{2}$ & -0.180 & 1.05 & 34.2 & 0.924 & 1.2 \\
$\mathrm{BBP}$ & $98 \% \mathrm{Ar}+2 \% \mathrm{O}_{2}$ & -0.252 & 1.23 & 27.2 & 0.964 & 0.7 \\
$\mathrm{BBP}$ & $95 \% \mathrm{Ar}+5 \% \mathrm{CO}_{2}$ & -0.216 & 1.07 & 25.9 & 0.954 & 0.8 \\
\hline
\end{tabular}

wavelengths within the spectral response of the detector. The performance of the ND filter was inferior to that of the BBP filter, probably a result of arc-length-independent radiation from the weld pool and electrode.

The best performance was achieved with the BBP filter (figs. 53 and 54). The BBP filter reduced radiation from the pool and electrode tip while providing an average measure of a wide range of wavelengths, as the ND filter did.

Figure 55 summarizes $R_{c}$ and $\sigma_{c}$ obtained with each combination of filter and shielding gas. For each optical filter, the standard deviation $\sigma_{c}$ was lower and the correlation coefficient $R_{c}$ was higher (better performance) for the $98 \% \mathrm{Ar}+2 \% \mathrm{O}_{2}$ shielding gas than for the $95 \% \mathrm{Ar}+5 \% \mathrm{CO}_{2}$ shielding gas. The poorer performance for the $95 \% \mathrm{Ar}+5 \% \mathrm{CO}_{2}$ shielding gas was attributed to the lower arc stability produced by $\mathrm{CO}_{2}$ additions to $\mathrm{Ar}$ [55]. The BBP filter provided the optimum results of arc-length estimation for both shielding gases. For the $98 \% \mathrm{Ar}+2 \% \mathrm{O}_{2}$, the uncertainty was $0.7 \mathrm{~mm}$, whereas the uncertainty for $95 \% \mathrm{Ar}+5 \% \mathrm{CO}_{2}$ was $0.8 \mathrm{~mm}$. 

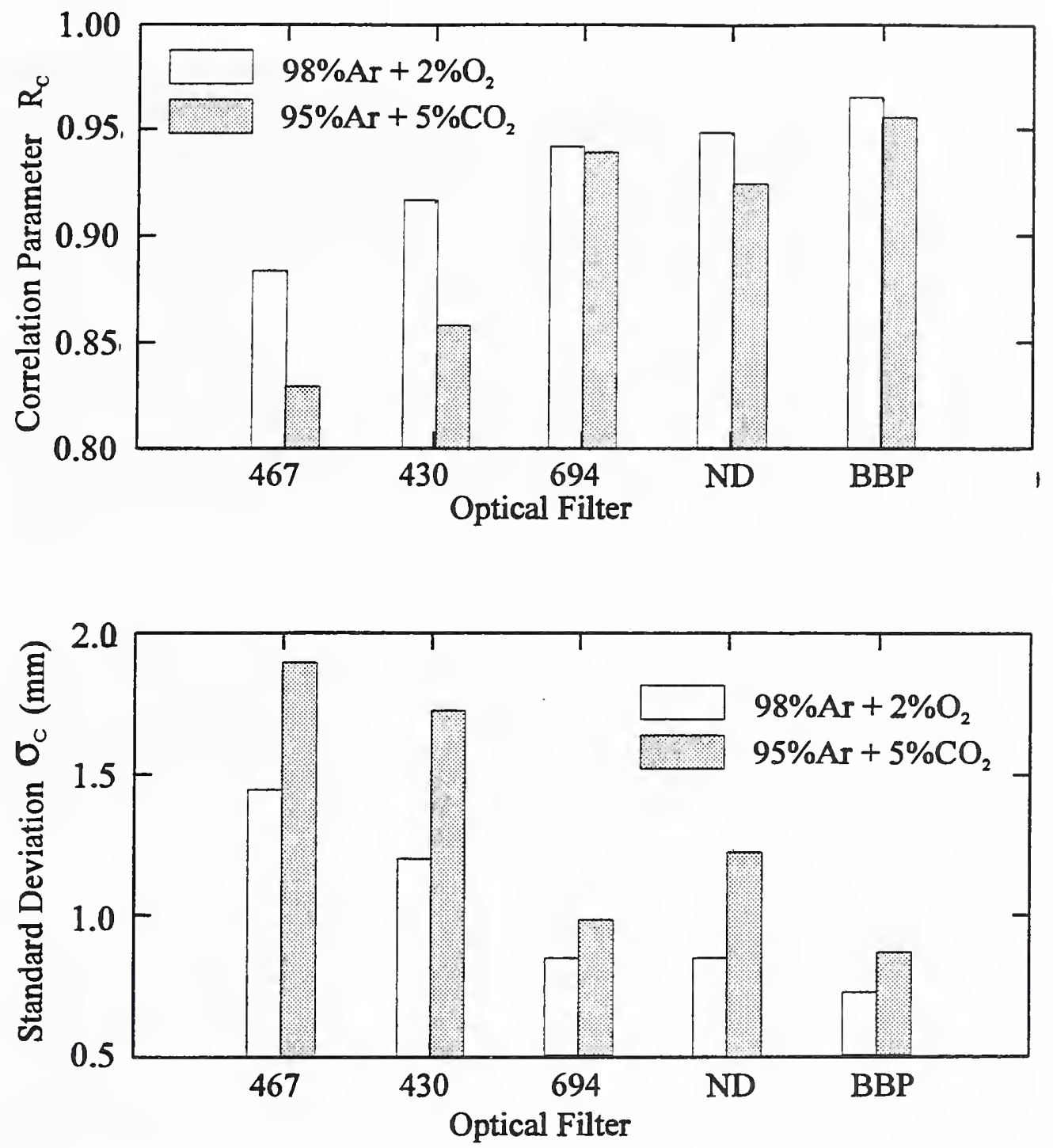

Figure 55. Summary of the correlation coefficient $R_{c}$ and standard deviation $\sigma_{c}$ for each combination of optical filter and shielding gas. Higher values of $R_{c}$ and lower values of $\sigma_{c}$. indicate better arc-length estimation. 


\subsection{Advantages and Disadvantages}

This arc-length-sensing technique depends only on arc-light intensity and arc current, and it does not require contact with the torch, electrode, or workpiece. The technique works independently of WFS, process voltage, and CTWD. Furthermore, the arc-light intensity can be obtained by a small detector that results in less intrusion into the welding area than techniques that use a proximity sensor or video camera. Arc-light sensing, as discussed in the following section, can simultaneously measure droplet-detachment frequency for process control.

The primary disadvantage of the technique is that it requires the use of an external sensor, the arc-light detector. Although the detector is small, it must be placed and maintained at a fixed distance from the welding arc. The technique requires that the sensor be calibrated by means of controlled welding in which the actual arc length is measured. Although the detector can be placed well away from the welding arc, it must have an unobstructed view of the arc, and it may be susceptible to weld metal spatter and fume.

\subsection{Summary}

Through-the-arc sensing for arc-length measurement is useful in some applications, but it suffers inherent limitations due to the composite nature of the process voltage. External sensors offer a more direct measure of arc length but have not found wide application because of the cost, size, and intrusiveness of the external sensor. Arc-length sensing as developed here uses a small external sensor, an arc-light-intensity detector, to estimate arc length.

Arc-light intensity increases with arc length for a given current and with current for a fixed arc length. An arc-length model incorporating simultaneous measurement of arc-light intensity and arc current was developed to obtain arc length [eq (43)].

The arc-length sensor provided the best estimate of arc length when the detector was in direct line of sight of the welding $\operatorname{arc}\left(\theta_{D}=0^{\circ}\right.$ in fig. 28) and when the lineof-sight axis was nearly parallel to the workpiece surface $\left(\theta_{S}=85^{\circ}\right.$ in fig. 28). The sensor operates over a large range of detector distances relative to the arc, but must remain fixed at the selected distance.

A BBP optical filter in front of the detector provided the best arc-length estimation. Arc length can be estimated within $0.7 \mathrm{~mm}$ for the $98 \% \mathrm{Ar}+2 \% \mathrm{O}_{2}$ shielding gas and within $0.8 \mathrm{~mm}$ for the $95 \% \mathrm{Ar}+5 \% \mathrm{CO}_{2}$ shielding gas.

The sensor must be recalibrated if the distance or orientation to the arc is changed. 


\section{Control of Arc Length}

\subsection{Background}

Extensive use of automatic-voltage-control (AVC) units in GTAW [56] attests the value of arc-length control in arc-welding processes. Arc length determines arc efficiency [38], power density [7], and pressure distribution [57], which, in turn, significantly influence weld geometry, microstructure, and mechanical properties. In GMAW, arc length also dictates transfer mode, arc stability, and deposition rate [58]. The random and unpredictable nature of disturbances to the welding process is another critical problem. For these reasons, automatic arc-length-control systems are essential.

In GTAW, the voltage measured is the voltage drop across a nonconsumable electrode and the arc, which is proportional to arc length; thus, by controlling the voltage, the length of the arc is controlled. In GMAW, the voltage measured is the composite voltage drop across the contact-tube/electrode interface, the electrode extension, and the arc (see fig. 4), and the arc voltage cannot be readily isolated from the other circuit voltages.

Different techniques are used to control GMAW arc length based on voltage sensing. One approach is to neglect the voltage drop across the electrode extension because it is typically smaller than the drop across the arc. A constant-current power source is used, and the power-source voltage drives the wire-feed motor in parallel with the arc. Changes in process voltage cause proportional changes in WFS and, thus, arc length. The most common approach is to use a constant-voltage power source with a fixed WFS [58]. The adjustments in current required to maintain constant voltage change the electrode melting rate [see eq (3)] and, hence, maintain a nearly constant arc length. Another approach uses a model of the voltage drop across the electrode extension derived from the WFS and the current [59]. The drop across the electrode is subtracted from the total voltage to obtain an estimate of the arc voltage. Either welding current or WFS is then adjusted to maintain constant arc length.

Other methods of arc-length control, based on arc-light-intensity measurements and similar to the approach used here, have been developed. In 1968 a patent was granted to Johnson and Sciaky for the use of arc-light intensity as a means of arclength control in GMAW [48]. The system has not been a commercial success, possibly because it is unable to discriminate the change in arc light due to variations in arc length from that due to changes in arc current. A similar system was developed for GTAW by Deam and Drew [51]. They developed a relationship between arc radiant intensity, arc current, and arc length and demonstrated an arc-length control based on the relationship. In another system devised by Romanenkov, arc-length control by sensing Ar-line intensity was reported to be especially useful in low-current GTAW where voltage sensing is not reliable [50].

The arc-length controller demonstrated here actuates the welding current to maintain arc length; thus, the response of the controller is not limited by a mechanical system, such as a wire feeder. Also, since the control system works independently of process voltage and WFS, it does not have the limitations of voltage-sensing systems, and it operates over a range of WFS settings. 


\subsection{Experimental Method}

Bead-on-plate welds were made on AISI-1020 plain-carbon steel with $98 \% \mathrm{Ar}$ $+2 \% \mathrm{O}_{2}$ shielding gas and a 1.2-mm-diameter ER100S-1 electrode. The travel speed was set at $6 \mathrm{~mm} / \mathrm{s}$. Welding power was supplied by the current regulator. Current and WFS were controlled by DAC signals from the computer. To characterize the welding-process transfer function, the computer produced a step change in the current command while simultaneously sampling the arc current, voltage, light intensity, and WFS. The actual arc length was measured simultaneously by using the video system. The controller was then designed and implemented in computer code. The controller performance was evaluated by having the computer introduce a step change in the arclength setpoint and in WFS while the controller was in operation. The video system recorded the arc length response during the disturbances.

\subsection{Arc-Length Sensing}

The arc length was estimated by using the detector to measure the arc-light intensity and the current transducer to measure the arc current. The detector position was fixed with $R_{D}=400 \mathrm{~mm}, \theta_{D}=0^{\circ}$, and $\theta_{S}=85^{\circ}$ (see fig. 28). The BBP optical filter was used on the detector (see fig. 30). Prior to welding, the arc-length sensor was calibrated by obtaining the actual arc length from video images. The calibration procedure yielded values for the coefficients $C_{0}, C_{1}$, and $C_{2}$, of the arc-length sensor equation, eq (43), which is repeated here:

$$
\ell_{A}=\frac{E_{D}-\left(\mathrm{C}_{0}+\mathrm{C}_{2} I_{A}^{2}\right)}{\mathrm{C}_{1} I_{A}}
$$

where $\ell_{A}$ is the estimated arc length, $E_{D}$ is the detector voltage, and $I_{A}$ is the arc current. The sensor response was considered to be static.

\subsection{Characterization of the Welding Process}

To characterize the open-loop static and dynamic properties of the welding process, step changes in welding current were used to perturb the process. Arc length, current, and light intensity were measured at $3000 \mathrm{~Hz}$ during the step changes.

Figure 56 shows the response of arc length to a step increase in current from 234 to $272 \mathrm{~A}$ with the WFS set at $123 \mathrm{~mm} / \mathrm{s}$. The arc length changes from 5.9 to $12.5 \mathrm{~mm}$; thus the gain of the welding process can be calculated as

$$
G_{w}=\frac{\Delta \ell_{A}}{\Delta J_{A}}=\frac{(12.5-5.9) \mathrm{mm}}{(272-234) \mathrm{A}}=0.17 \mathrm{~mm} / \mathrm{A},
$$

where $\Delta \ell_{\mathrm{A}}$ is the change in arc length and $\Delta I_{A}$ is the change in arc current.

The change in arc length with respect to time was modeled as an exponential rise of the form 


$$
\ell_{A}=\ell_{0}+\Delta \ell_{A} e^{\left(-\frac{1}{\tau_{w}} t\right)},
$$

where $\ell_{A}$ is the instantaneous arc length, $\ell_{0}$ is the initial arc length, $\Delta \ell_{A}$ is the overall change in arc length, $\tau_{w}$ is the process time constant, and $t$ is time. Regression analysis of the arc length data yielded $\tau_{w}=0.13 \mathrm{~s}$ (the time required to reach 63.2 percent of the final arc-length value). The solid line in figure 56 represents the modeled arclength response of eq (49). The transfer function for the welding process can, therefore, be written as

$$
K_{W}=\frac{\Delta \ell_{A}}{\Delta J_{A}}=\frac{G_{w}}{\left(\tau_{w} s+1\right)},
$$

where $s$ is the Laplace-transform variable. The process is characterized as a linear first-order system that is amenable to proportional-integral-derivative (PID) control [18]. The time constant $\tau_{w}$ and gain $G_{w}$ are assumed to be constant over the range of WFS and current that produces spray transfer.

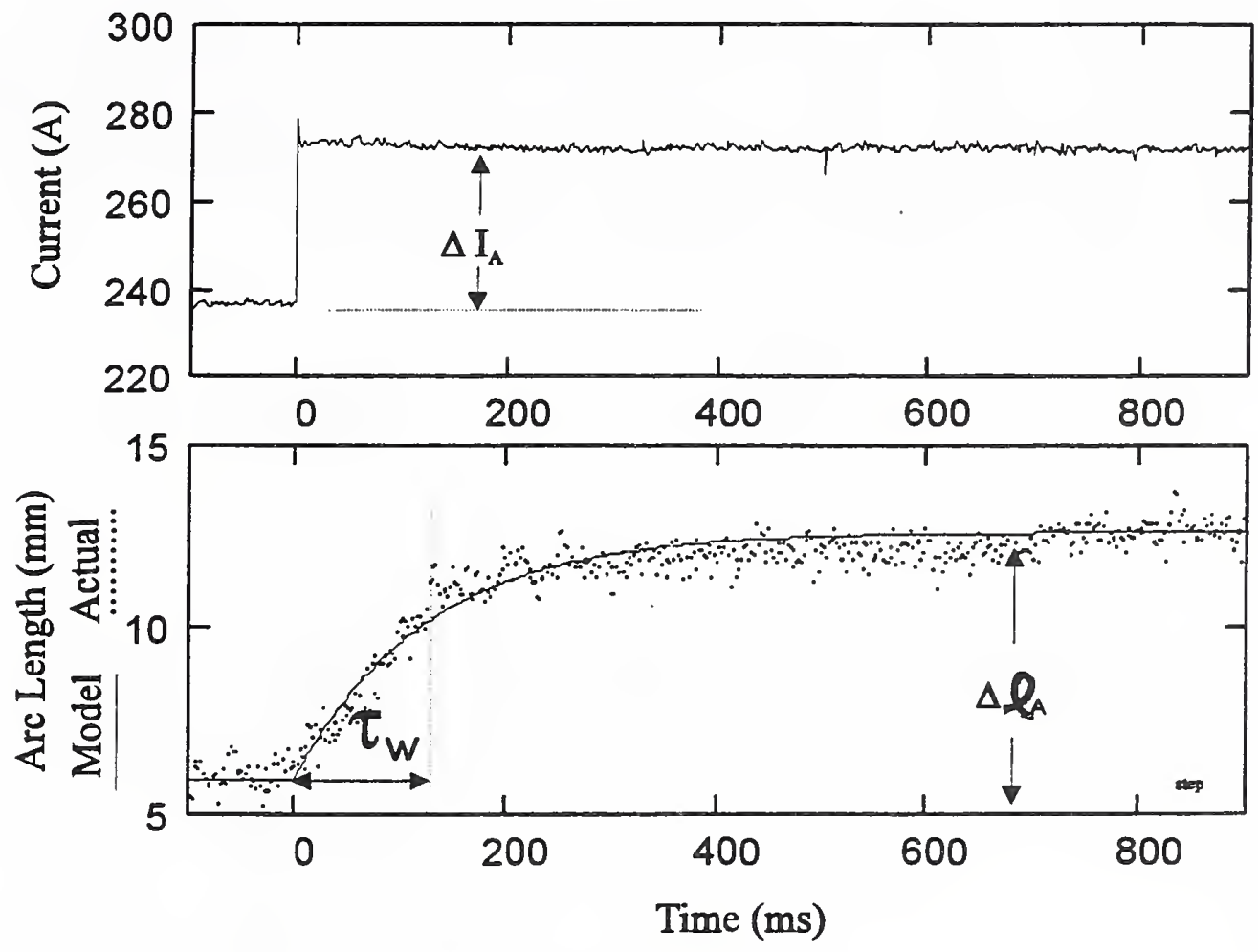

Figure 56. Response of arc length to a step increase in arc current for determining the static and dynamic characteristics of the welding process. The WFS was $123 \mathrm{~mm} / \mathrm{s}$. The solid curve shows the mathematical model. 


\subsection{Design of the Controller}

The overall system block diagram is shown in figure 57. Here, the final output of the process is considered to be the arc length. The arc length is estimated from the intermediate outputs, the actual arc current, and the detector voltage. The required inputs to the system are nominal values of CTWD, WFS, and the desired arc length $\ell_{\mathrm{D}}$. The nominal current estimator uses the equation developed by Lesnewich [4] to estimate the nominal or feedforward current $I_{F F}$ from

$$
\mathrm{WFS}=\mathrm{a} I_{F F}+\mathrm{b} I_{F F}^{2} \ell_{e}
$$

where $\mathrm{a}$ and $\mathrm{b}$ are constants for the steel electrode, $\ell_{e}$ is the electrode extension, and $\ell_{e}=$ CTWD $-\ell_{D}$. Calculation of the feedforward current prior to welding provided a method for obtaining the initial welding clirrent by biasing the controller output.

The arc-length controller is a PID controller, implemented in the software of the computer, that calculates the output welding current as

$$
I_{w}(k)=K_{P} \Delta \ell(K)+K_{I} \sum_{k} \Delta \ell(k)+K_{D}[\Delta \ell(k)-\Delta \ell(k-1)]+I_{F F},
$$

where $K_{P}, K_{I}$, and $K_{D}$ are the proportional, integral, and derivative gains, $\Delta \ell$ is the length error $\left(\ell_{D}-\ell_{A}\right)$, and $k$ represents the discrete sample period. To tune the controller, that is, to select controller-gain values, a system model was derived by using the closed-loop transfer function obtained from eqs (50) and (52). The controller gains $K_{P}, K_{I}$, and $K_{D}$, were selected to minimize arc-length overshoot and settling time by using the time-domain transient response predicted by the model $[18,19]$. The gains $K_{P}, K_{I}$, and $K_{D}$ were set to $1.6,10$, and $0.5 \mathrm{~A} / \mathrm{mm}$, respectively.



Figure 57. Block diagram of the arc-length control system. 
To operate the arc-length controller, the current and detector signals were lowpass filtered with a cut-off frequency of $40 \mathrm{~Hz}$. The computer sampled the detector and current signals and calculated the estimated arc length from eq (47). The estimated arc length and controller gains were then used in eq (52) to calculate the welding current. The controller was executed at a rate of $100 \mathrm{~Hz}$.

\subsection{Results and Discussion}

The performance of the system was tested by introducing step changes to the arc-length setpoint and WFS. The modeled and actual responses of the arc length to a 50-percent step increase in arc-length setpoint, from 6 to $9 \mathrm{~mm}$, are shown in figure 58. The WFS was constant at $135 \mathrm{~mm} / \mathrm{s}$. The arc length reached 63.2 percent of its final value in $0.07 \mathrm{~s}$, had a maximum overshoot to $9.7 \mathrm{~mm}$, and settled within 5 percent of the setpoint within $0.5 \mathrm{~s}$. The current response was similar, changing from 240 to $258 \mathrm{~A}$ with a peak overshoot to $265 \mathrm{~A}$. As expected, the integral action of the controller provided a steady-state error of 0 for the step increase in length setpoint.

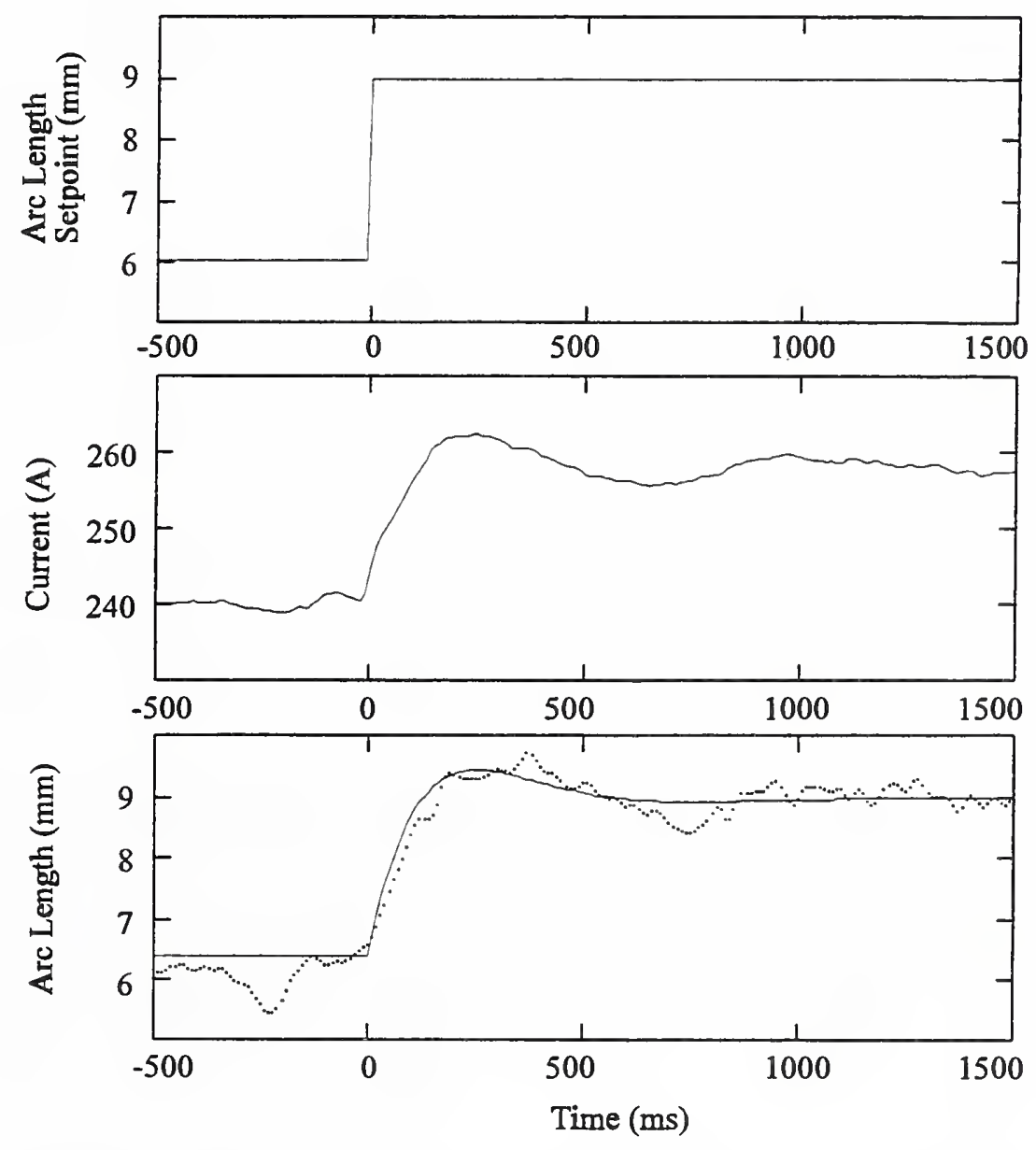

Figure 58. Closed-loop response of the control system to a step increase in arc-length setpoint from 6 to $9 \mathrm{~mm}$. The WFS was $135 \mathrm{~mm} / \mathrm{s}$. The solid curve shows the mathematical model. 
The response of the controller to a disturbing input in the form of a step increase in WFS from 140 to $170 \mathrm{~mm} / \mathrm{s}$ is shown in figure 59. The arc length showed a transient decrease to $4.8 \mathrm{~mm}$ from the setpoint value of $8 \mathrm{~mm}$. (The current changed from 252 to $280 \mathrm{~A}$.) The arc length recovered to the setpoint value within $0.5 \mathrm{~s}$, with a steady-state error of 0 . For comparison, the response of the constant-voltage (CV) power source, without the current regulator, is shown in figure 60 . The $\mathrm{CV}$ power source had a static output slope of $-2 \mathrm{~V} / 100 \mathrm{~A}$. The current settled to $270 \mathrm{~A}$ from an initial value of $250 \mathrm{~A}$ in approximately $0.38 \mathrm{~s}$. The actual arc length changed from 7 to $5 \mathrm{~mm}$ for a steady-state error of $2 \mathrm{~mm}$.

The controller is capable of providing a steady-state error of zero in response to step changes in setpoint and WFS. For the controller gains selected, the transient response of the controller to a step change in setpoint $(0.07 \mathrm{~s})$ is faster than the natural response of the process $(0.13 \mathrm{~s})$. For disturbing inputs in the form of step changes

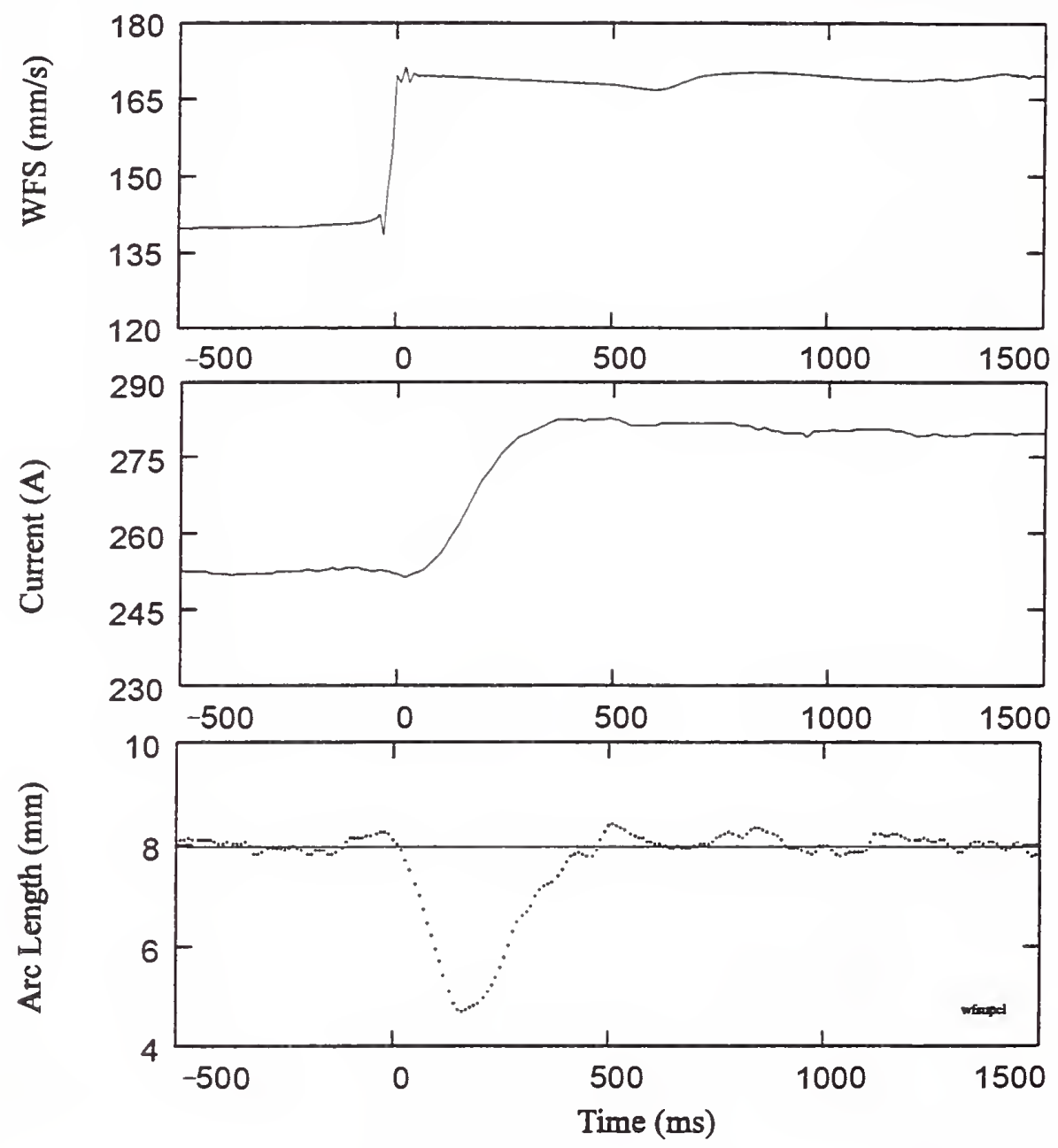

Figure 59. Closed-loop response of the control system to a disturbing input in the form of a step increase in WFS from 140 to $170 \mathrm{~mm} / \mathrm{s}$. The solid curve shows the mathematical model. 


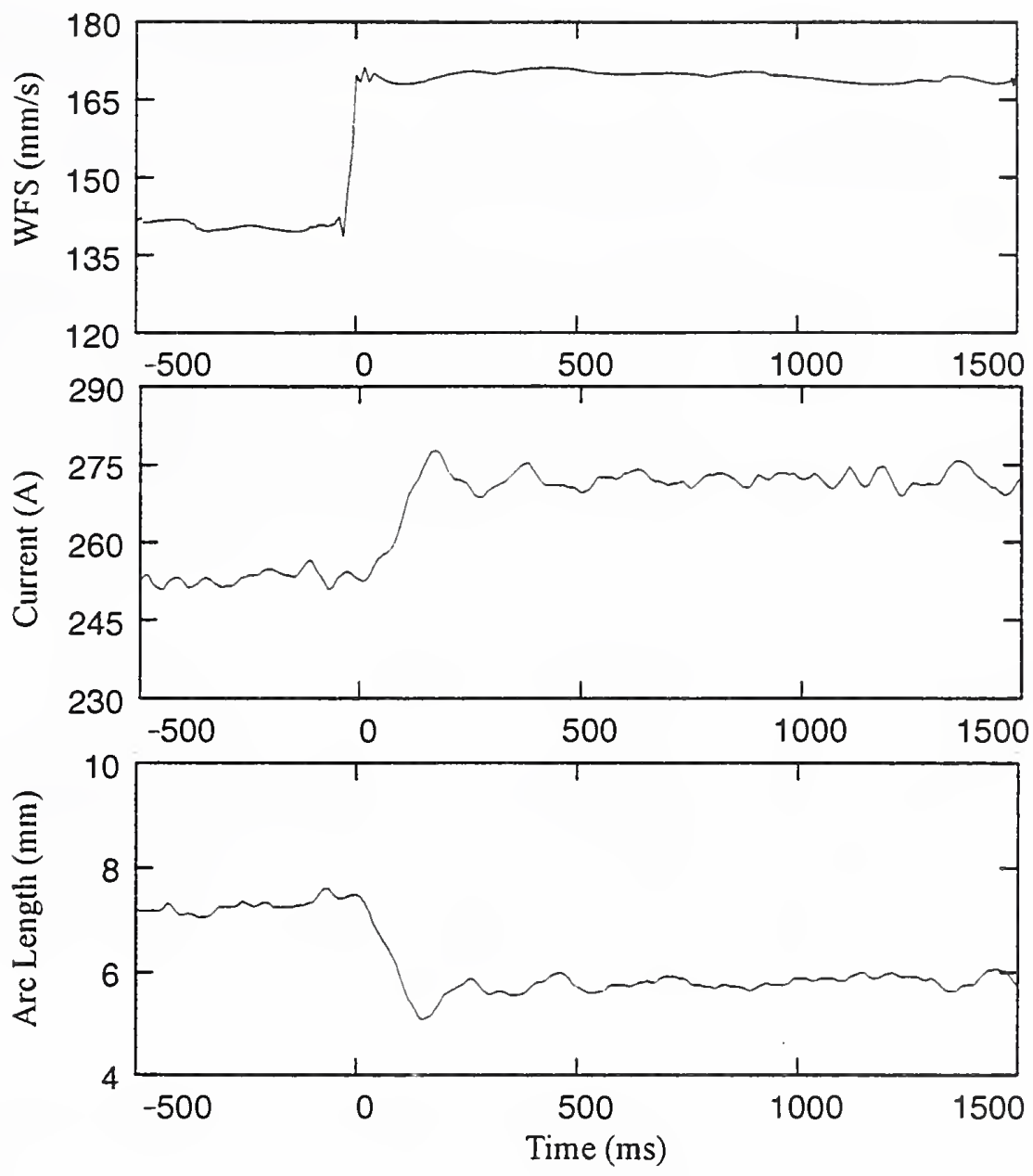

Figure 60. Response of a typical CV power source to a disturbing input in the form of a step increase in WFS from 140 to $170 \mathrm{~mm} / \mathrm{s}$.

in WFS, the transient response of the controller can be limited by the assumption of constant process coefficients and fixed controller gains. The transient response can be improved by the use of an adaptive controller, in which the controller gains vary, depending on the nominal values of current and WFS [60]. The response of the controller is also limited by the dynamic range of the arc-light detector. A higher current would increase the rate of change of arc length; however, beyond a current of about $300 \mathrm{~A}$, the detector voltage saturated.

\subsection{Summary}

The composite nature of the process voltage in GMAW makes it difficult to control arc length directly from process-voltage measurements. Maintaining constant voltage does not guarantee constant arc length, since the voltage must be adjusted to accommodate changes in WFS. 
An arc-length controller for spray-transfer GMAW using an arc-length sensor based on arc-light intensity and current has been demonstrated. The controller is a simple, fixed-gain PID type in which the gains have been selected on the basis of experimentally determined process characteristics. The model parameters are assumed to be constant over the range of current and WFS values for spray transfer. The controller changes the arc current to maintain arc length.

Results showed that the controller was capable of providing a zero steady-state error in response to step changes in setpoint and WFS. Although the transient performance of the controller was satisfactory, it could be improved by using an adaptive controller. The primary advantage of the technique is that it is independent of process voltage and WFS. A disadvantage is its reliance on an external sensor, the arc-light detector, which intrudes into the weld area. Also, the arc-length sensor must be calibrated, and the calibration must be maintained in the harsh welding environment.

\section{Detection of Droplet-Transfer Frequency}

The mode of metal transfer during GMAW influences process stability, weld-pool behavior, penetration, solidification, and heat flow, all of which affect weld quality [8]. Thus, it is desirable to develop a means of measuring droplettransfer mode and frequency to monitor or even control the welding process in real time. This section describes the use of arc-light sensing for detecting the dropletdetachment frequency during spray-transfer welding.

\subsection{Background}

The primary transfer modes in GMAW have been defined as short-circuit, globular, and spray [61]. Each mode exhibits a transfer frequency that is characteristic and definitive of that mode. The spray-transfer mode requires the use of an active shielding gas and, usually, higher WFS and currents than those associated with either short-circuit or globular transfer. Spray transfer can be subdivided into drop- and streaming-spray modes, differentiated by the size of the droplets. Drop spray occurs when the droplets are nominally the same diameter as the electrode. At higher WFS and current, streaming-spray transfer occurs where the droplet diameters are smaller than that of the electrode wire.

Several techniques for sensing droplet-transfer mode and frequency have been developed for GMAW based on process-voltage and current sensing, through-the-arc sensing $[62,63]$, or airborne acoustic sensing $[8,64,65]$. These techniques have been successfully demonstrated for real-time detection of transfer mode and frequency during short-circuiting and globular-transfer conditions. Under these welding conditions, large amplitude variations occur in both the arc length and electrode extension as the droplets transfer. The variations in arc length and electrode extension impose significant and readily detectable fluctuations in the process voltage, current, and arc acoustic signals. During drop- and streaming-spray transfer, however, the fluctuations in these signals due to droplet detachment become more subtle: they approach or become smaller than the natural power-source ripple. As a result, sensing the process 
voltage, current, or arc acoustic signals is not a robust means of monitoring droplet detachment in real time during spray-transfer welding.

Preliminary research [66] showed that the use of static-transfer-mode maps could be used to replace real-time sensing of droplet transfer during spray transfer. Figure 61 shows the transfer modes associated with average values of process voltage and current that were obtained from postweld inspection of high-speed videos. The term static was used to indicate that all welding parameters except the process voltage, current, and WFS (CTWD, gas and electrode compositions, and voltage sensing location) remained static or fixed for each transfer-mode map. The problems with this technique are (1) it requires the off-line analysis of a large number of welding conditions to develop the map and (2) the location of data points within the map depends on the voltage and current calibrations, CTWD, electrode extension, shielding gas and electrode compositions, and contact-tube condition, some of which naturally vary during welding.

Additional preliminary research demonstrated that arc-light sensing can be used for droplet-frequency detection [52]. The purpose of the work presented here was to determine the mechanism by which the droplet-detachment signature was imposed on the arc-light signal and to optimize a technique for providing real-time detection of droplet-transfer frequency during spray-transfer welding.

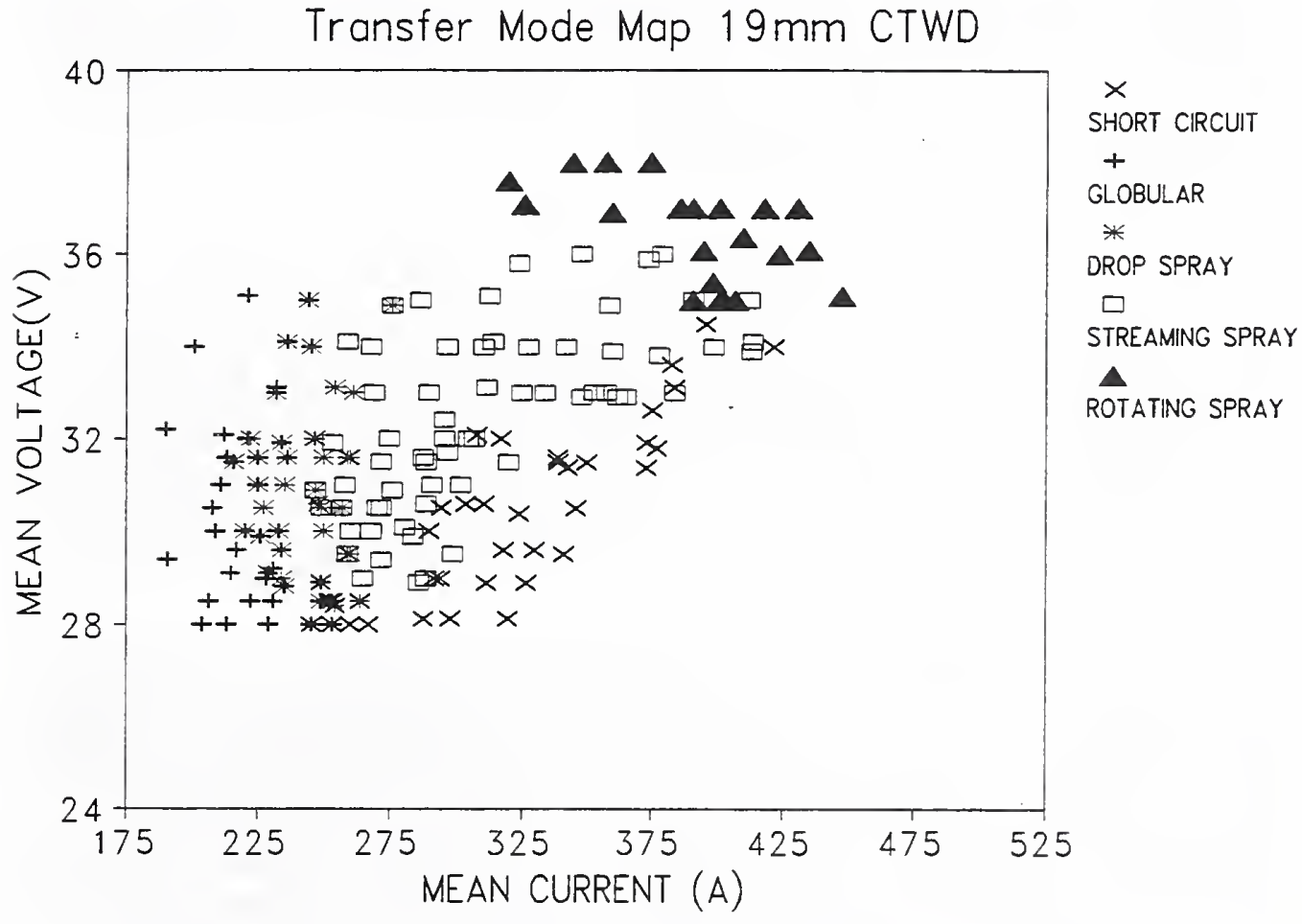

Figure 61. Static-transfer-mode map developed for determining the droplettransfer mode based on average current and voltage (from Heald [66]). 


\subsection{Experimental Method}

This section describes the welding conditions, arc-light-detector configuration, and the methods of data collection and analysis used for determining droplet-transfer frequency. A complete description of the welding equipment, data-acquisition computer, and laser-backlighting and video system was given in section 4 .

\subsubsection{Welding Conditions}

Bead-on-plate welds were made on AISI-1020-steel strips with an ER100S-1 steel electrode. Either $98 \% \mathrm{Ar}+2 \% \mathrm{O}_{2}$ or $95 \% \mathrm{Ar}+5 \% \mathrm{CO}_{2}$ was used as the shielding gas. The travel speed was set at $6 \mathrm{~mm} / \mathrm{s}$, and the CTWD was $19 \mathrm{~mm}$. The welding current, voltage, and WFS were selected to provide a spray-transfer mode. A transistorized current regulator provided constant current, and a conventional SCR power source without the regulator provided constant voltage.

\subsubsection{Detector Configuration}

The detector position was fixed with $R_{D}=400 \mathrm{~mm}, \theta_{D}=0^{\circ}$, and $\theta_{S}=85^{\circ}$ (see fig. 28). The influence of detector orientation and position on droplet-frequency detection was not investigated because this detector position was considered the best for simultaneous arc-length estimation.

To evaluate the effect of optical filtering on droplet-frequency detection, the ND, BBP, 430-nm, 694-nm, and 467-nm filters were sequentially placed in front of the detector. The optical-transmission profile and the corresponding arc-emission spectrum for each filter are shown in figures 29 through 33. For each optical filter, welds were made with both shielding gases.

\subsubsection{Data Collection}

During each weld, the arc current, process voltage, WFS, and detector voltage signals were sampled at $3000 \mathrm{~Hz}$ by the data-acquisition computer. All signals were low-pass filtered with a cut-off frequency of $1000 \mathrm{~Hz}$. The $1000 \mathrm{~Hz}$ cut-off frequency was sufficiently below the $1500-\mathrm{Hz}(3000 / 2 \mathrm{~Hz})$ Nyquist frequency required to prevent aliasing [67]. Simultaneously, video images of the weld area, with and without laser backlighting, were taken at $3000 \mathrm{frames} / \mathrm{s}$. An electronic link synchronized data collection between the data-acquisition computer and the high-speed video system. Data from each weld were collected for $4 \mathrm{~s}$.

The actual droplet frequency is contained in the change in electrode extension produced each time a droplet detaches from the end of the electrode. To obtain an accurate measure of the electrode extension, the magnification of the video camera lens was adjusted such that the electrode extension, from the end of the contact tube to the top of the arc, filled the entire video screen. After each weld, the electrode extension was extracted from the video image with an image processor. A sequence of processed images, showing the electrode extension with laser backlighting, is shown in figure 62 . To process each image, a horizontal reference line was placed at the end of the contact tube. Then three vertical lines were scanned from the horizontal reference line, along the electrode, until the endpoints were found. The electrode extension was then calculated as the average length of the three vertical lines. An 


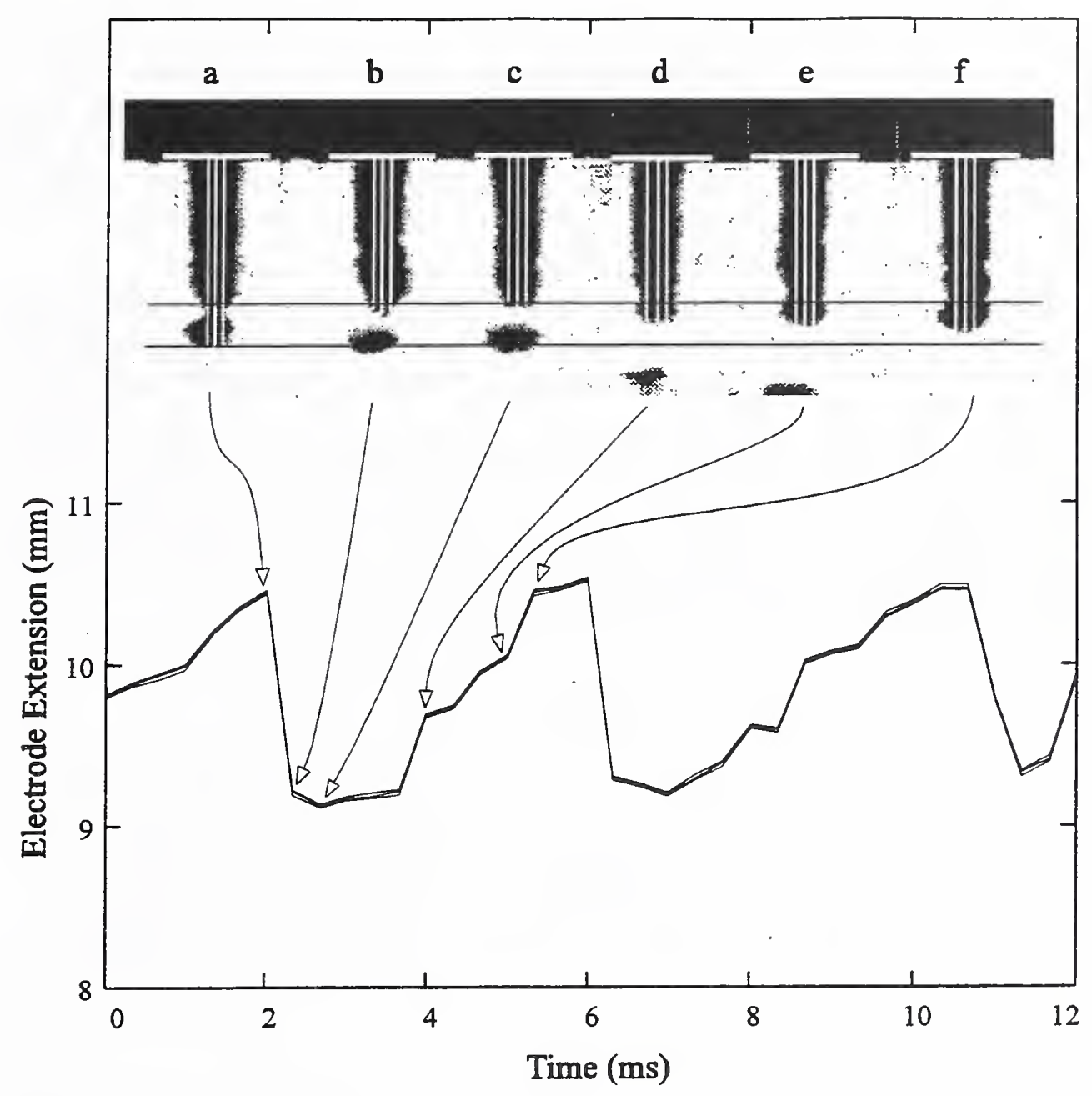

Figure 62. Video-image processing used to obtain the reference or actual droplet-transfer frequency. The change in electrode extension, as the droplet detaches, produces a sawtooth waveform from which the transfer frequency can be readily obtained.

electrode extension measurement was obtained for each measurement of current, voltage, WFS, and detector voltage.

To investigate the mechanism responsible for generating the droplet-detachment signature within the arc-light-detector signal, video images were taken of the arc without laser backlighting. The magnification of the video-camera lens was adjusted such that the arc filled the entire video screen. The electrode extension was measured for each frame, as described in the previous section. The electrode extension was then correlated to the detector voltage and to the appearance of the arc and droplet as the droplet increased in size, detached, and passed through the arc. 


\subsubsection{Analysis of Droplet-Transfer Frequency}

To determine the frequency components of the current, voltage, detector-voltage and electrode-extension time traces, the power spectral density (PSD) for each was computed [67]. The PSD transform expresses the amplitude of power contained within the signal as a function of frequency (the power spectrum). The amplitude within a frequency interval in the power spectrum corresponds directly to the amplitude of that frequency component in the time-domain signal.

An average spectrum was calculated for each time-domain signal obtained during a weld. First, the time-domain signals were high-pass filtered with a digital filter at $80 \mathrm{~Hz}$ to remove the $\mathrm{dc}$ and low-frequency components. Next, 4096 data points were windowed by using a Hamming function to reduce spectral leakage. Then, an intermediate PSD was calculated and stored for that time-domain window. The window was then moved forward in the time-domain data by 1024 points, and the procedure was repeated until the end of the data was reached. Finally, an average spectrum was calculated from the intermediate spectra and then smoothed with a moving-box average. The frequency resolution $F_{R}$ (in hertz) for the PSD is

$$
F_{R}=\frac{\text { sample frequency }}{\text { data window }}=\frac{3000}{4096}=0.73 \text {. }
$$

The interest in using the PSD calculation was to determine the frequency of the droplet detachment and to compare the spectra from each signal (current, process voltage, detector voltage, and electrode extension) under a variety of welding conditions and detector optical filters. To enable direct comparison, each spectrum was normalized by dividing all amplitudes in the range 80 to $500 \mathrm{~Hz}$ (the expected transfer-frequency range) by the maximum amplitude within that frequency range.

\subsubsection{Analysis of Optical Filters}

To determine the influence of optical filtering on transfer-frequency detection, a normalized detector-voltage ratio was calculated for each optical filter under the same welding conditions. With the welding conditions fixed, the dc and ac amplitudes of light emitted by the arc can be assumed to be constant from weld to weld. Furthermore, the electronic gain of the detector/amplifier and the gain of the ADC were fixed. Thus, the only gain changed in the detector system was that produced by the different optical filters.

The normalized detector-voltage ratio $\xi_{D}$ was calculated from the time-domain detector-voltage signal $E_{D}$ provided by each optical filter. First, the standard deviation of the signal $E_{D}$ (std) was found; it is proportional to the ac amplitude of the signal. Next, the mean of the signal $E_{D}$ (mean) was calculated; it is analogous the dc component of the signal. Finally, $\xi_{D}$ was calculated as

$$
\xi_{D}=\left[\frac{E_{D}(\mathrm{std})}{\left.E_{D} \text { (mean }\right)}\right] \times 100 \% .
$$

Large values of $\xi_{D}$ indicate that the corresponding wavelengths passed by the optical filter were strongly influenced by droplet detachment. 


\subsection{Results and Discussion}

The following sections discuss the measurements of the actual frequency of droplet detachment, the correlation of the detector-voltage signal to droplet detachment, and the effect of detector optical filtering on droplet-frequency detection.

\subsubsection{Measurement of Droplet Detachment}

A sequence of electrode-extension images and the corresponding electrodeextension measurements is shown in figure 62. The diameter of the droplet was $1.08 \mathrm{~mm}$, the droplet frequency was $222 \mathrm{~Hz}$, and the WFS was $130 \mathrm{~mm} / \mathrm{s}$ [see eq (55)]. At point a, a droplet was about to detach from the electrode and the electrode extension was at a maximum. At point $b$, the droplet had left the electrode, causing an abrupt decrease in the electrode extension. The molten liquid at the electrode tip began to recoil from surface-tension forces. At point $c$, the molten electrode tip had fully recoiled and the electrode extension was at a minimum. Through points $\mathrm{d}$, e, and $\mathrm{f}$, the continuous advancement of the electrode produced a nearly linear increase in electrode extension with time. Simultaneously, the tip of the electrode continued to melt and the next droplet grew to the point of detachment (point a). The periodic droplet detachment resulted in a sawtooth waveform of the electrode extension from which the actual droplet-detachment frequency could be readily obtained by using its PSD.

\subsubsection{Correlation of Droplet Detachment with Arc-Light-Detector Voltage}

A series of arc images during a droplet-detachment cycle and the corresponding detector-voltage and electrode-extension measurements is shown in figure 63. Note that the arc images were obtained without the use of laser backlighting, and they are displayed as negative images (the darker portions of the images correspond to the brighter, more intense portions of the arc). The detector-voltage waveform had a sawtooth appearance, similar to that of the electrode extension waveform but approximately $180^{\circ}$ out of phase.

At point a in figure 63, the droplet was about to detach from the electrode and the detector voltage was at a minimum. At point $b$, the droplet had detached and the detector voltage had increased abruptly. As the droplet moved away from the electrode (point $c$ ), the detector voltage reached a maximum. Through points $d, e$, and $f$, the growth of the next droplet provided a nearly linear decrease in detector voltage with time until the next droplet detached. Again, the periodic droplet detachment resulted in a sawtooth waveform of the detector voltage from which the dropletdetachment frequency could be readily obtained by using its PSD.

Several mechanisms are hypothesized as the cause of the observed change in detector voltage during droplet detachment:

1. The droplet reduces the temperature of the arc as it grows and detaches from the electrode tip. As the droplet enters the arc, the surface of the droplet boils, releasing additional Fe vapor into the arc [68]. The increased concentration of Fe ions and electrons provides sufficient conductivity to support the arc current at reduced temperatures. The reduction in temperature causes a corresponding reduction in radiant intensity. 
2. The droplet blocks the radiation from the arc volume on the side of the droplet opposite the detector. The blockage causes a reduction in radiation reaching the detector.

3. The cycle of droplet growth and detachment causes a change in arc length. The change in arc length causes a corresponding change in arc volume and, hence, in detector voltage.

The first mechanism cannot be easily verified, either analytically or experimentally. However, as discussed below, the evidence that the largest change in detector voltage occurred while using the $430-\mathrm{nm}$ filter offers some support for this mechanism. Mechanism two is also difficult to verify because the amount of arc radiation blocked by the droplet as it passes through the arc is difficult to quantify. The third mechanism can be evaluated with existing data.

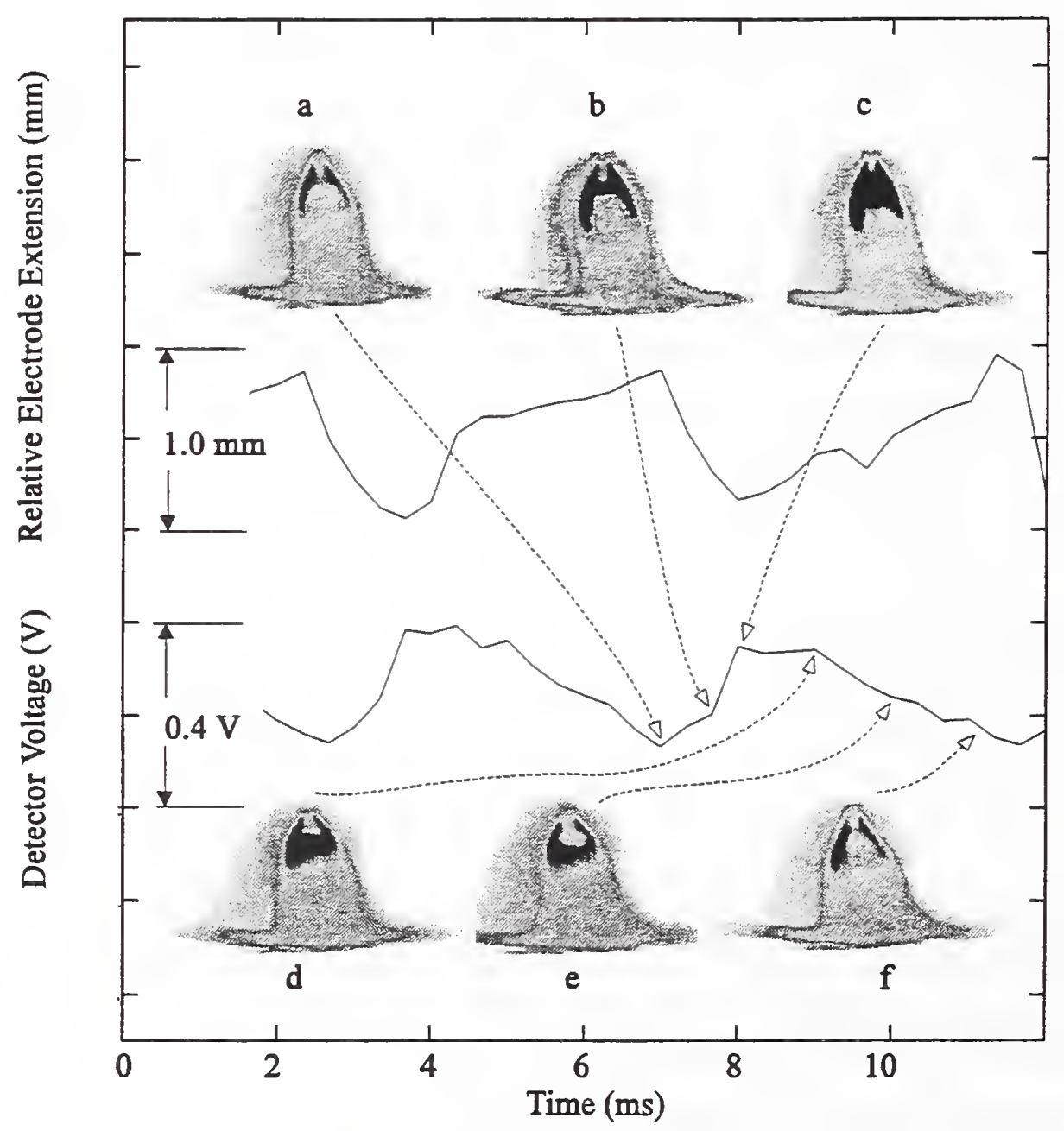

Figure 63. Correlation of droplet detachment with arc-light-detector voltage. The sawtooth waveform of the detector-voltage signal is similar to that of the electrode-extension signal but shifted in phase by $180^{\circ}$. 
By conservation of electrode volume and an assumption of spherical droplets, the actual droplet diameter $d_{d}$ can be calculated from the WFS, the electrode diameter $d_{e}$, and the droplet-transfer frequency $f_{d}$ by

$$
d_{d}=\left[\frac{3 \mathrm{WFS} d_{e}^{2}}{2 f_{d}}\right]^{\frac{1}{3}}
$$

From the conditions used to obtain figure 63 (WFS $=130 \mathrm{~mm} / \mathrm{s}, f_{d}=222 \mathrm{~Hz}$, and $\left.d_{e}=1.2 \mathrm{~mm}\right), d_{d}$ was determined to be $1.08 \mathrm{~mm}$. The relationship between arc length $\ell_{A}$, arc current $I_{A}$, and detector voltage $E_{D}$ was expressed in eq (42), which is repeated here:

$$
E_{D}=\mathrm{C}_{0}+\mathrm{C}_{1} \ell_{A} I_{A}+\mathrm{C}_{2} I_{A}^{2}
$$

where $C_{0}, C_{1}$, and $C_{2}$ are calibration coefficients. Differentiating eq (56) with respect to $\ell_{\mathrm{A}}$ gives

$$
d E_{D}=\mathrm{C}_{1} I_{A} d \ell_{A}
$$

Assuming the change in arc length $d \ell_{A}$ is equal to the droplet diameter $d_{d}$, then $d_{d}$ can be substituted directly into eq (57). By using $C_{1}=1.23 \times 10^{-3} \mathrm{~V} /(\mathrm{mm} \cdot \mathrm{A})$ from table 2 and $I_{A}=255 \mathrm{~A}$, the change in detector voltage was found to be $0.34 \mathrm{~V}$, within 15 percent of the $0.4-\mathrm{V}$ amplitude shown in figure 63 . Thus, the droplet detachment signature in the detector-voltage signal can probably be attributed to the change in arc length as the droplet detaches. It is possible, however, that all three of the proposed mechanisms play some part in imposing the droplet-detachment signature on the detector-voltage waveform.

\subsubsection{Detection of Droplet-Transfer Frequency: Experiments}

Figure 64 shows time traces of the current, process voltage, detector voltage, and electrode extension for a spray-transfer weld made by using $98 \% \mathrm{Ar}+2 \% \mathrm{O}_{2}$ shielding gas, the BBP optical filter, a WFS of $130 \mathrm{~mm} / \mathrm{s}$, and a constant current of $255 \mathrm{~A}$ from the current regulator. The detector-voltage and electrode-extension traces show the sawtooth waveform associated with droplet detachment. The process-voltage waveform contains features that can be associated with droplet detachment. The current regulator gives no indication of droplet detachment in the current waveform.

The PSD plots for each waveform in figure 64 are shown in figure 65. Again, each spectrum has been normalized by the maximum amplitude within the frequency range 80 to $500 \mathrm{~Hz}$. The electrode-extension spectrum shows a maximum amplitude at the actual droplet-transfer frequency of $222 \mathrm{~Hz}$, with no other significant peaks in the spectrum. The droplet diameter was $1.08 \mathrm{~mm}$. The detector-voltage spectrum also shows a maximum at the droplet-transfer frequency of $222 \mathrm{~Hz}$, and it displays small peaks at the power-source line frequencies of 120 and $360 \mathrm{~Hz}$. The current and process-voltage spectra both have maximum amplitudes at $120 \mathrm{~Hz}$ with smaller peaks at 180 and $360 \mathrm{~Hz}$, all associated with the power-source line frequency. The current 
and process-voltage spectra also have peaks at the droplet-transfer frequency. However, without prior knowledge of the true transfer frequency, it would be difficult to identify the transfer frequency simply by observing the current or process-voltage spectra.

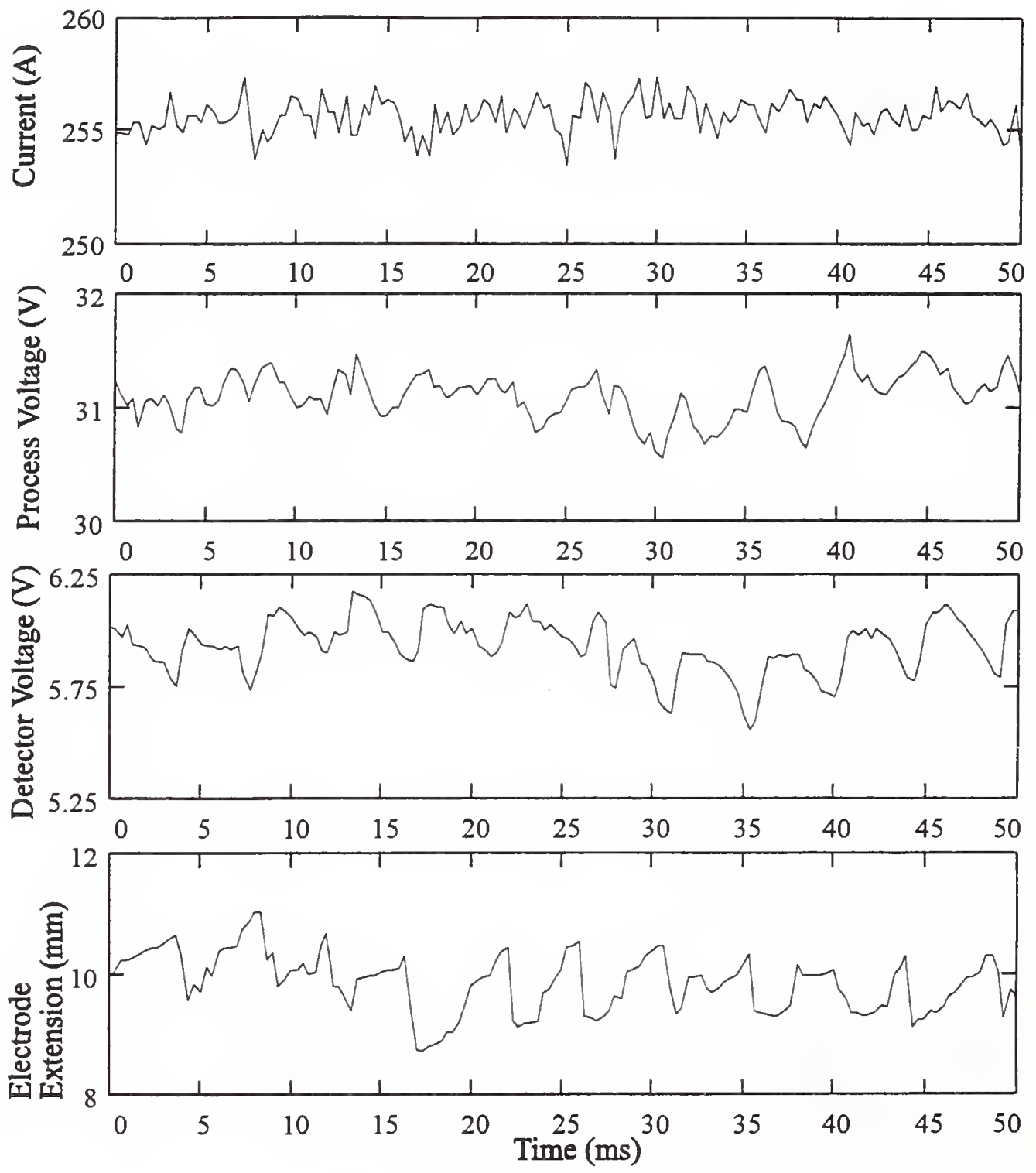

Figure 64. Time traces of the current, process voltage, detector voltage, and electrode extension for a spray-transfer weld made by using the current regulator. Droplet-detachment features are present in the detector-voltage, electrodeextension, and process-voltage traces but not in the current trace. Optical filter: BBP. Welding conditions: $98 \% \mathrm{Ar}+2 \% \mathrm{O}_{2}, 255 \mathrm{~A}, 130 \mathrm{~mm} / \mathrm{s}$. 


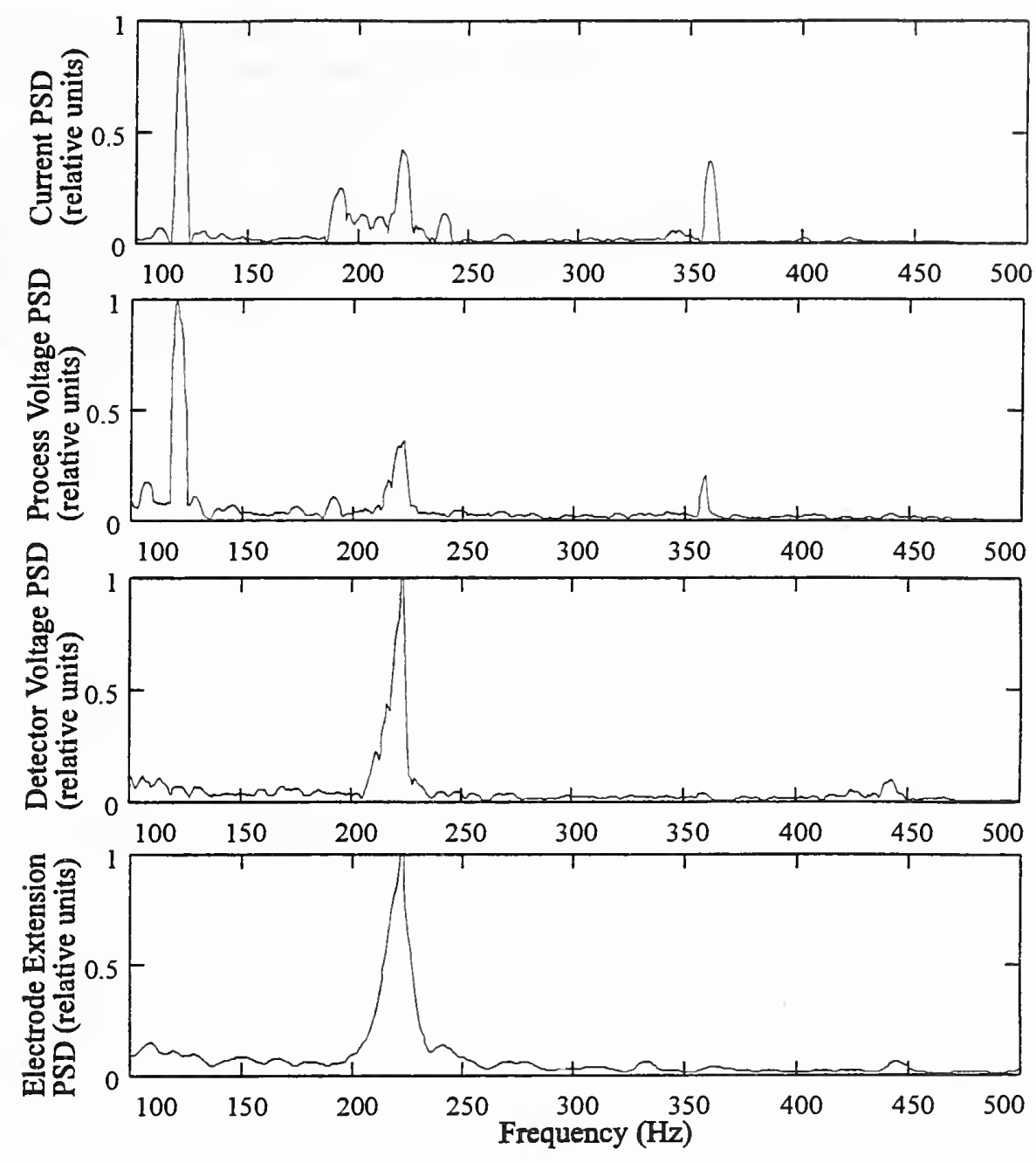

Figure 65. PSD plots for the weld in figure 64. The electrode-extension, and detector-voltage spectra show the actual droplet-transfer frequency of $222 \mathrm{~Hz}$. The process-voltage and current spectra show peaks at the droplet-transfer frequency and at the power-source line frequencies of 120 (maximum), 180, and $360 \mathrm{~Hz}$.

Figure 66 shows time traces of the current, process voltage, detector voltage and electrode extension for a streaming-spray-transfer weld made by using $98 \% \mathrm{Ar}$ $+2 \% \mathrm{O}_{2}$ shielding gas, the $430-\mathrm{nm}$ optical filter, a WFS of $180 \mathrm{~mm} / \mathrm{s}$, and a constant current of $320 \mathrm{~A}$ from the current regulator. The detector-voltage and electrodeextension traces show the sawtooth waveform associated with droplet detachment. Neither the process voltage nor current traces contain features that can be linked with certainty to droplet detachment. 
The PSD plots for the time traces of figure 66 are shown in figure 67 . The electrode-extension spectrum shows that the actual transfer frequency was $397 \mathrm{~Hz}$, corresponding to a droplet diameter of $0.99 \mathrm{~mm}$. The maximum in the detectorvoltage spectrum also occurred at $397 \mathrm{~Hz}$, with a small peak at $360 \mathrm{~Hz}$. Most of the significant peaks in the current and voltage spectra are, again, related to the powersource line frequency, and the maximum has shifted to $360 \mathrm{~Hz}$. A small peak occurs in both current and process-voltage spectra at the transfer frequency. These data indicated that the arc-light-detector signal could be employed to detect the droplet-transfer frequency during drop- and streaming-spray conditions during welding using the current regulator.
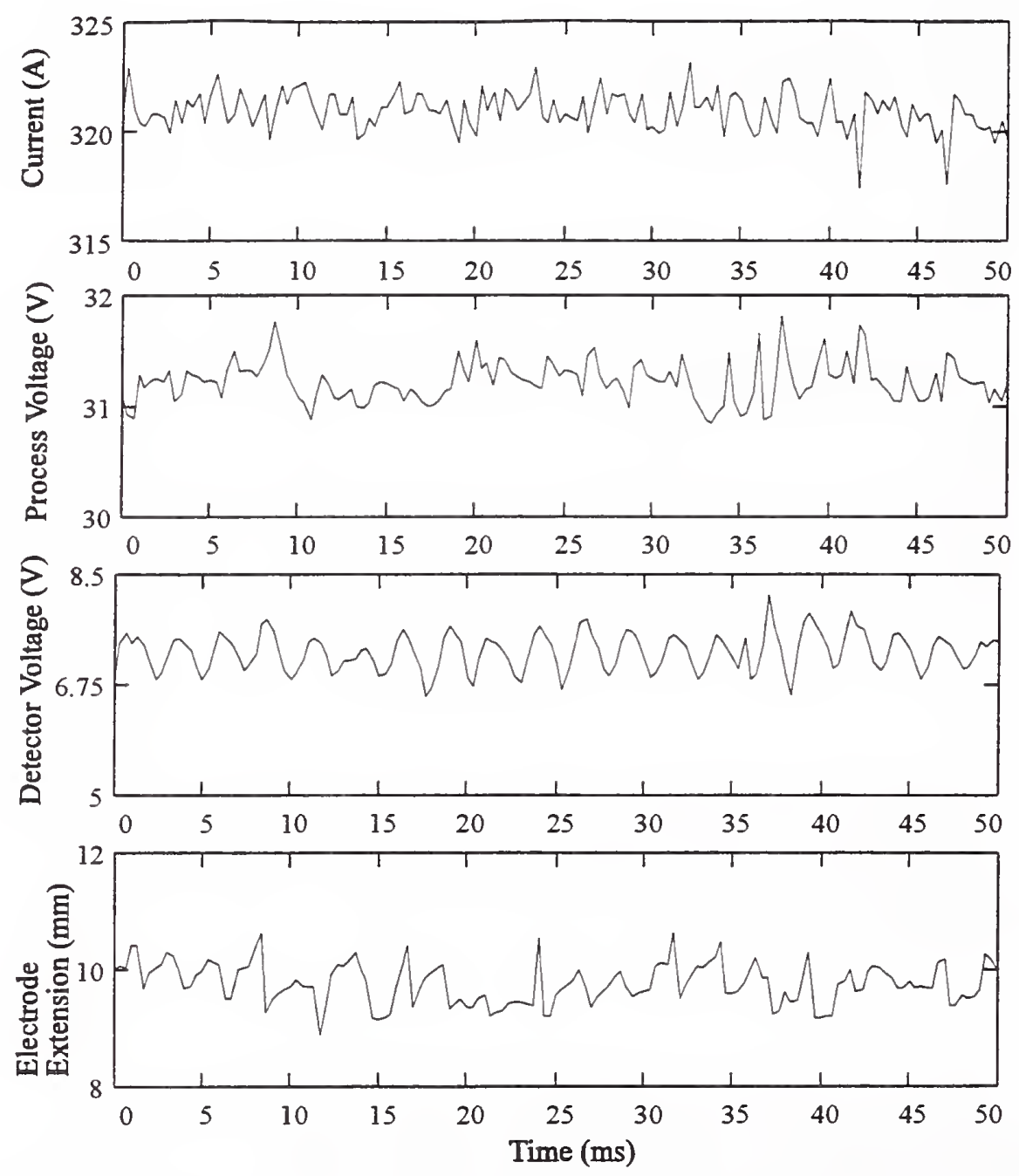

Figure 66. Time traces of the current, process voltage, detector voltage, and electrode extension for a streaming-spray-transfer weld made by using the current regulator. The detector voltage and electrode extension show the droplet-detachment signature. Neither the process voltage nor the current show the droplet-detachment features. Optical filter: $430 \mathrm{~nm}$. Welding conditions: $98 \% \mathrm{Ar}+2 \% \mathrm{O}_{2}, 320 \mathrm{~A}, 180 \mathrm{~mm} / \mathrm{s}$. 


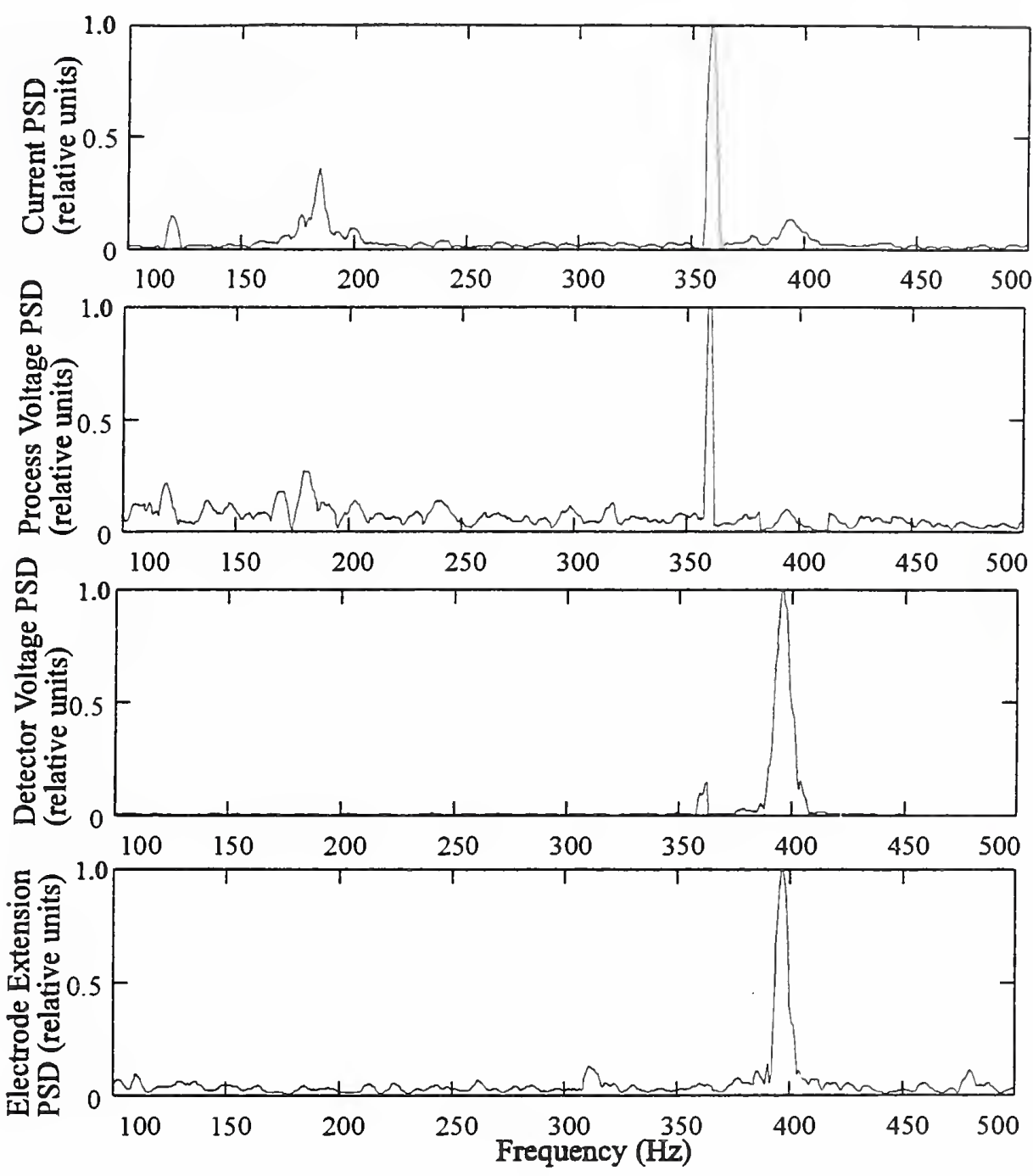

Figure 67. PSD plots for the weld in figure 66. The electrode-extension and detector-voltage spectra show the actual droplet-transfer frequency of $397 \mathrm{~Hz}$. Peaks in the current and voltage spectra are related to the powersource line frequency ( $360 \mathrm{~Hz}$ maximum), and small peaks occur in both spectra at the transfer frequency.

Figure 68 shows the time traces of a weld made by using the conventional SCRcontrolled, CV power source. The nominal voltage was set at $32.5 \mathrm{~V}$ and the WFS was $144 \mathrm{~mm} / \mathrm{s}$ resulting in a mean current of $282 \mathrm{~A}$. The shielding gas was $98 \% \mathrm{Ar}$ $+2 \% \mathrm{O}_{2}$ and the 430-nm optical filter was used. The electrode-extension and detector-voltage waveforms readily show the droplet-detachment signature. The current and process-voltage traces also show variations that may be linked to droplet detachment, though the additional power-source ripple makes the connection less certain. 


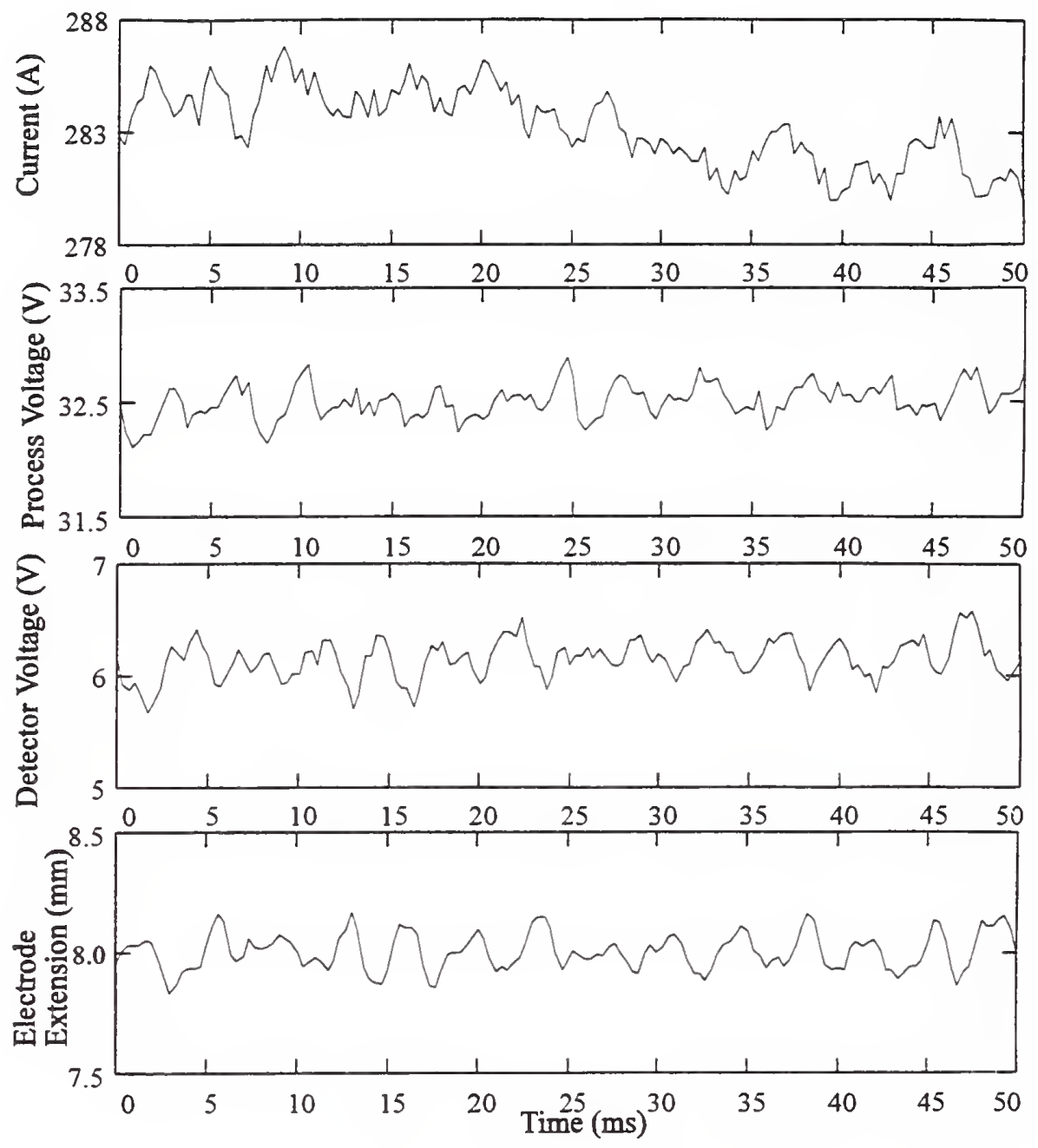

Figure 68. Time traces of the current, process voltage, detector voltage, and electrode extension for a spray-transfer weld made by using the $\mathrm{CV}$, SCR power source. The electrode-extension and detector-voltage traces show the droplet-detachment signature. The current and process-voltage traces show droplet detachment confounded by the power-source ripple. Optical filter: $430 \mathrm{~nm}$. Welding conditions: $98 \% \mathrm{Ar}+2 \% \mathrm{O}_{2}, 32.5 \mathrm{~V}, 144 \mathrm{~mm} / \mathrm{s}$.

The PSD plots for the time traces of figure 68 are shown in figure 69 . The electrode-extension spectrum shows that the actual transfer frequency was $272 \mathrm{~Hz}$, corresponding to a droplet diameter of $1.05 \mathrm{~mm}$. The maximum in the detector voltage spectrum also occurred at $272 \mathrm{~Hz}$, with a small peak at $360 \mathrm{~Hz}$. The current and voltage spectra contain peaks associated with the power-source line frequency with a maximum at $360 \mathrm{~Hz}$. Both also contain a large number of significant peaks that can be related to the variation in SCR firing angle as the power source attempted to maintain a constant voltage output [69]. A small peak occurs in both current and 
process-voltage spectra at the transfer frequency; however, it is not possible to determine the true transfer frequency from either spectrum. From these results, we concluded that the variations in detector voltage due to droplet detachment, under the welding conditions used here, were significantly greater than those caused by the power source current and voltage ripple. Also, arc-light sensing may provide a means of monitoring droplet frequency when welding with conventional CV, SCR power sources.
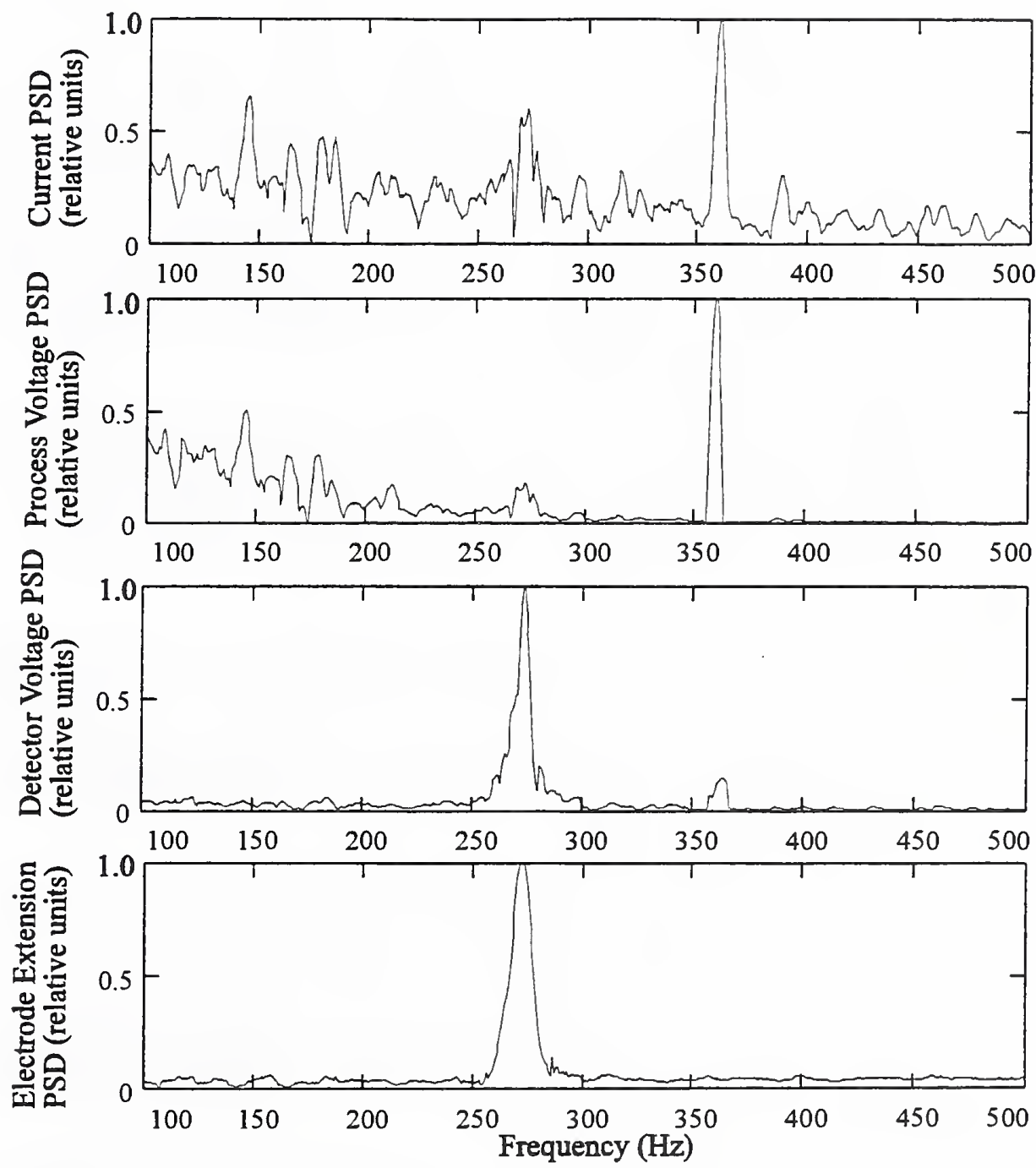

Figure 69. PSD plots for the weld in figure 68. The electrode-extension and detector-voltage spectra show the actual droplet-transfer frequency of $272 \mathrm{~Hz}$, with a smaller peak at $360 \mathrm{~Hz}$ in the detector-voltage spectrum. The current and voltage spectra contain power-source line frequency peaks (maximum at $360 \mathrm{~Hz}$ ) and a large number of significant peaks related to the SCR power-source characteristics. 
Without supplemental information, such as the expected transfer-frequency range, it is not possible to identify the actual droplet-transfer frequency during spray welding by observing the process-voltage or current time traces and power spectra alone. Also, under the welding conditions used here, the amplitude of the droplet frequency, as observed from the detector-voltage spectra, was significantly larger than that caused by the power-source ripple. The actual droplet-transfer frequency can be obtained by locating the frequency of maximum amplitude within the detector-voltage spectrum.

\subsubsection{Effect of Optical Filtering}

The normalized detector-voltage ratio $\xi_{D}$ for each combination of shielding gas and optical filter is shown in figure 70. A constant current of $255 \mathrm{~A}$ was provided by the current regulator, and the WFS was $130 \mathrm{~mm} / \mathrm{s}$. For both shielding gases, the largest value of $\xi_{D}$ occurred when using the $430-\mathrm{nm}$ filter, indicating it offers the best droplet-frequency detection. The 430 -nm filter passes a range of wavelengths associated with several Fe emission lines (see fig. 31), supporting the hypothesis that the higher value of $\xi_{D}$ results from a significant change in $\mathrm{Fe}$ concentration within the arc during the cycle of droplet growth and detachment.

Although the other filters are adequate for droplet-frequency detection, no other performance trend was evident among the filters between the two shielding gases. This result indicates that there may be a variety of arc emission changes occurring during droplet detachment that depend upon the shielding-gas composition.

\subsection{Summary}

The actual droplet frequency could be easily obtained by measuring the change in electrode extension from high-speed video recording of the process. The periodic droplet detachment produced a sawtooth waveform in the electrode-extension and detector-voltage time traces, with the detector-voltage trace shifted in phase by $180^{\circ}$ from that of the electrode-extension trace.

Several mechanisms were hypothesized as the cause of the observed change in detector voltage during droplet detachment. The mechanisms may include a change in arc temperature and, hence, arc radiant intensity; a blockage of a portion of the radiation on the side of the droplet opposite the detector; or a change in arc length and, hence, arc-radiation volume. By assuming spherical droplets, calculating the droplet size from the WFS and droplet frequency, and using eq (43) for arc-length estimation, the droplet-detachment signature in the detector-voltage signal was shown to be attributable to changes in arc length.

Under the welding conditions used here, the variations in detector voltage due to droplet detachment were significantly greater than those caused by power-source current and voltage ripple when using either the current regulator or a conventional, $\mathrm{CV}, \mathrm{SCR}$ power source.

Without supplemental information, such as the expected transfer-frequency range, it was not possible to identify the actual droplet-transfer frequency during spray welding by observing the process-voltage or current time traces or power spectra individually. However, we concluded that the actual droplet-transfer frequency 

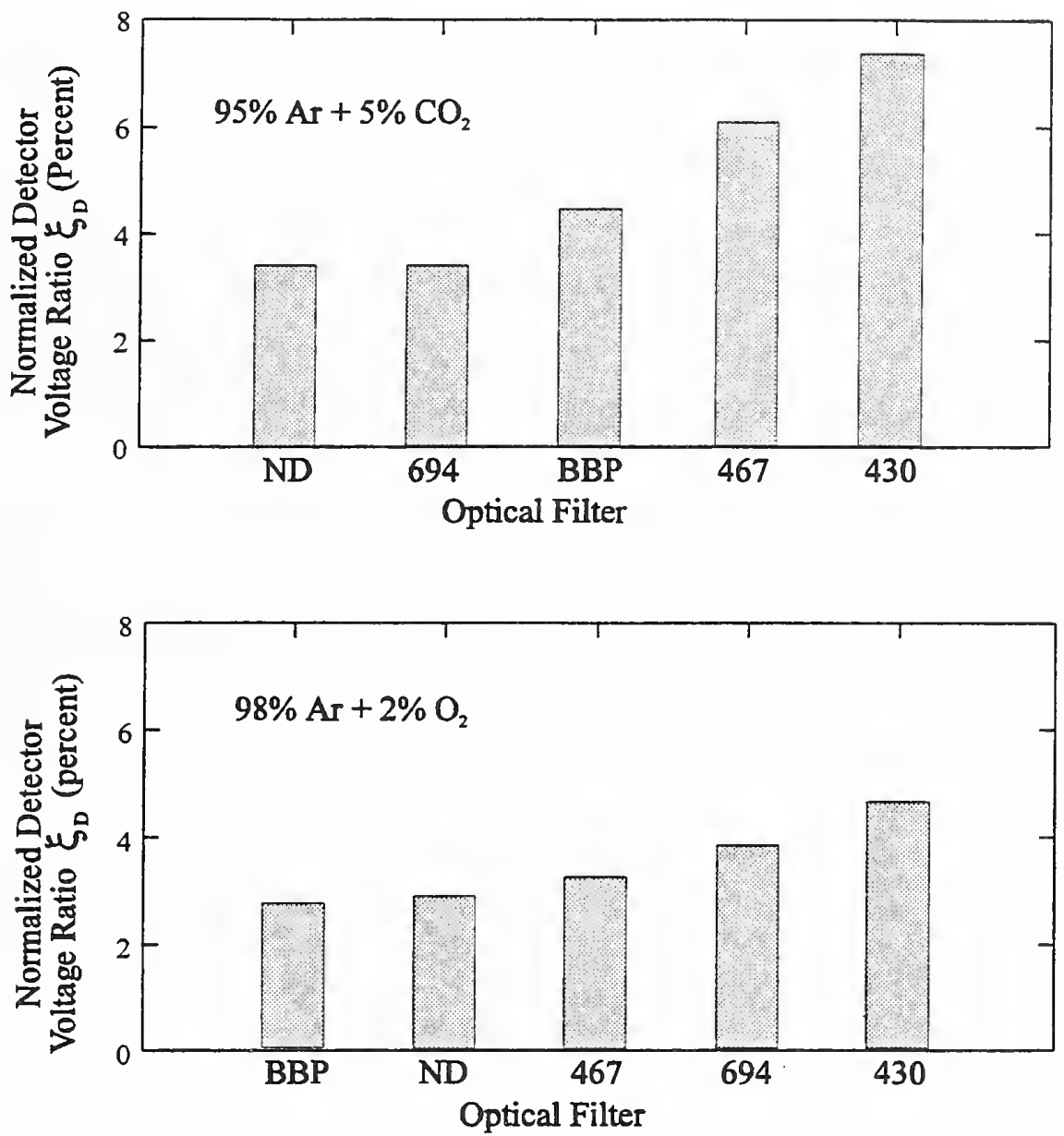

Figure 70. The normalized detector-voltage ratio $\xi_{D}$ for each combination of shielding gas and optical filter. For both shielding gases, the maximum $\xi_{D}$ occurred when the $430-\mathrm{nm}$ filter was used, indicating that this filter offers the best droplet-frequency detection. Other welding conditions: $255 \mathrm{~A}, 130 \mathrm{~mm} / \mathrm{s}$.

can be obtained in real time by locating the frequency of maximum amplitude within the detector-voltage PSD.

For both the $98 \% \mathrm{Ar}+2 \% \mathrm{O}_{2}$ and the $95 \% \mathrm{Ar}+5 \% \mathrm{CO}_{2}$ shielding gases, the maximum value of the normalized detector-voltage ratio $\xi_{D}$ occurred when using the 430-nm filter. The 430-nm filter, which passed a range of wavelengths associated with several Fe-emission lines, offered the best droplet-frequency detection.

The technique developed here offers the capability of monitoring droplet frequency in real time. It could be used to map the transfer mode and frequency over a broad range of welding conditions. Furthermore, the ability to detect droplet-transfer frequency could provide a real-time measure of process stability or form the basis for controlling droplet-transfer frequency during welding. Using only arc-light measurements, the method is independent of voltage, current, WFS, and type of power source. 


\section{Control of Droplet-Transfer Frequency}

\subsection{Background}

The mode of metal transfer during GMAW depends on many factors, including arc current, process voltage, WFS, electrode extension, CTWD, and shielding gas and electrode compositions. The metal-transfer mode determines process stability and, therefore, weld quality by influencing weld-pool behavior (penetration, solidification, and heat flow) [8]. The ability to measure and control droplet frequency may improve weld quality in automatic welding [63].

The work by Lesnewich [70] was instrumental in classifying transfer modes as short-circuiting, globular, and spray transfer, in order of increasing arc current. The spray-transfer mode can be subdivided into drop and streaming spray, where the diameters of the metal droplets are equal to and less than the diameter of the electrode, respectively. The mode of metal transfer in this study is confined to spray transfer, since previous work has shown that the ability to measure and control shortcircuiting and globular transfer can be accomplished via current and voltage sensing $[8,62,63]$.

For a given shielding gas composition, electrode composition, and electrode diameter, droplet-transfer frequency is typically expressed as a function of the welding current. Under spray-transfer conditions, the general trend observed is a nearly linear increase in droplet frequency with current $[62,71]$. Given a suitable sensor, a simple controller should be capable of controlling transfer frequency.

\subsection{Experimental Method}

Bead-on-plate welds were made on AISI-1020-steel strips with an ER100S-1 steel electrode and $98 \% \mathrm{Ar}+2 \% \mathrm{O}_{2}$ shielding gas. The travel speed was set at $6 \mathrm{~mm} / \mathrm{s}$, and the CTWD was $19 \mathrm{~mm}$. Welding power was provided by the current regulator. The welding current and WFS were controlled by DAC signals from the computer and were selected to produce the spray-transfer mode.

To characterize droplet frequency as a function of welding parameters, the WFS and current were ramped through a range of values. The current, WFS, and detector voltage signals were collected at $3000 \mathrm{~Hz}$ by the data-acquisition computer and stored for off-line analysis. All signals were low-pass filtered with a cut-off frequency of $1000 \mathrm{~Hz}$. The $1000 \mathrm{~Hz}$ cut-off frequency was sufficiently below the $1500 \mathrm{~Hz}(3000 / 2 \mathrm{~Hz})$ Nyquist frequency required to prevent aliasing [67]. Simultaneously, video images were recorded and analyzed to determine electrode extension. After welding, the detector-voltage data were processed to extract transfer frequency. The transfer frequency was then correlated with the welding parameters.

The transfer-frequency sensor and controller were then designed and implemented in computer code. The controller performance was evaluated in response to a step change in transfer-frequency setpoint with the controller actuating either current or current and WFS. 


\subsection{Droplet-Transfer-Frequency Sensor}

From the results reported in the previous section, it was determined that the droplet transfer frequency could be obtained simply by searching for the frequency with maximum amplitude in the detector PSD (see section 7.3.3). The detector position was fixed with $R_{D}=400 \mathrm{~mm}, \theta_{D}=0^{\circ}$, and $\theta_{\mathrm{S}}=85^{\circ}$ (see fig. 28). The 430-nm optical filter placed in front of the detector (see fig. 31).

The detector voltage was band-pass-filtered in the range 80 to $500 \mathrm{~Hz}$ with an analog filter. Use of the analog filter eliminated the need to high-pass-filter the detector signal in the computer software. The computer continuously sampled the detector voltage signal at $3000 \mathrm{~Hz}$. Each sample was stored in the next available location of a ring buffer set up in computer memory. Because the ring buffer was used, the computer did not have to wait for an entire window of data before calculating the PSD. The ring buffer incorporates some history into the signal, in that some of the data points used for the last PSD are included in the present PSD. The history effect tended to smooth the frequency signal, which was useful because the dropletfrequency signal tended to be noisy.

The execution rate of the PSD code is determined by the speed of the computer and the data-window length. The PSD calculation here required a data-window length that is a power of 2 [67]. The data-window length was set at 2048 points; therefore, the frequency resolution for the sensor $F_{R}$ was

$$
F_{R}=\frac{\text { sample frequency }}{\text { window length }}=\frac{3000 \mathrm{~Hz}}{2048}=1.46 \mathrm{~Hz} \text {. }
$$

Equation (58) shows that the frequency sensor had an inherent dead band equal to the frequency resolution. That is, only changes in transfer frequency greater than the resolution could be detected. The data window selected was a compromise between frequency resolution and processing speed. The computer was able to calculate the PSD in $0.166 \mathrm{~s}$, or it could provide a frequency measurement at a rate of about $6 \mathrm{~Hz}$.

\subsection{Characterization of the Process}

The process was characterized in three ways: (1) The current was ramped from 250 to 300 A over a period of $20 \mathrm{~s}$ with constant WFS and CTWD. (2) The WFS was ramped from 120 to $170 \mathrm{~mm} / \mathrm{s}$ with constant current and CTWD. (3) Both the current and WFS where ramped with constant CTWD. The WFS was set to eq (3) for each value of current.

Droplet frequency and electrode extension as a function of current for two constant values of WFS are shown in figure 71. The transfer frequency increased linearly with current for both WFS settings. The slopes of the two lines, however, are different $(4.6 \mathrm{~Hz} / \mathrm{A}$ for WFS $=140 \mathrm{~mm} / \mathrm{s}$ and $2.1 \mathrm{~Hz} / \mathrm{A}$ for WFS $=145 \mathrm{~mm} / \mathrm{s})$. These data suggest that a shorter electrode extension or lower WFS provides a greater increase in droplet frequency with current. Figure 71 showed that for a given WFS, droplet frequency is a linear function of current and could be controlled by the actuating current. 


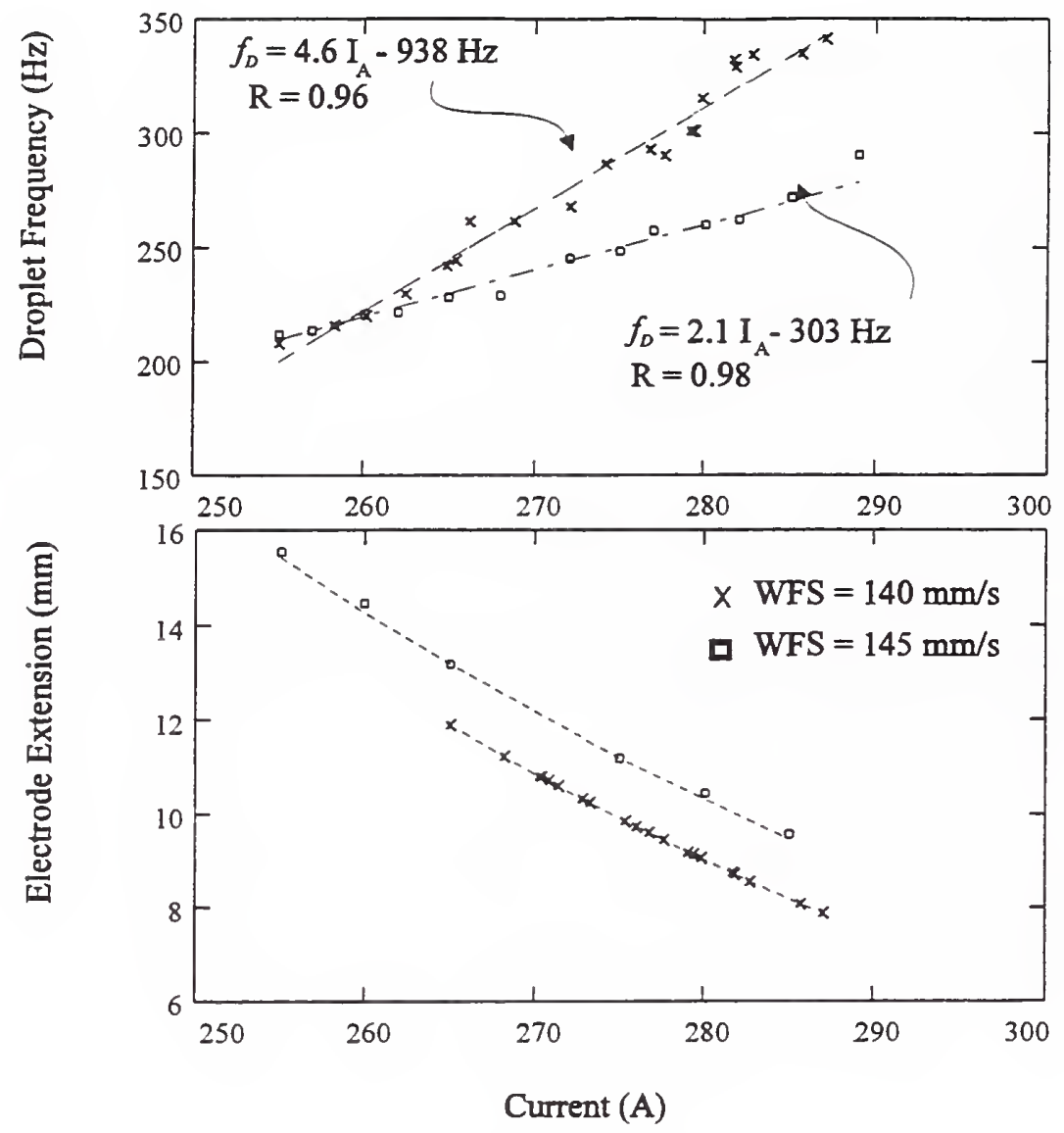

Figure 71. Droplet-transfer frequency and electrode extension as functions of current for two values of constant WFS.

The process transfer function can be expressed as

$$
K_{W}=K_{W}(\mathrm{WFS}) \text {. }
$$

The droplet-transfer frequency and electrode extension as a function of WFS for two settings of constant current are shown in figure 72. Droplet frequency exhibits a nonlinear, nonmonotonic response to increasing WFS and electrode extension for both current levels. Evidently, electrode extension, WFS, or both significantly alter the droplet-detachment mechanism with fixed arc current. These data indicate that a simple, linear controller could not be applied to control droplet-transfer frequency by actuating only WFS.

The droplet-transfer frequency as a function of both current and WFS is shown in figure 73. The WFS was slaved to the current according to eq (3), a condition that yields approximately constant electrode extension. The general trend observed was an increase in droplet frequency with increasing current and WFS. The rate of increase, however, appears to be a function of electrode extension $(3.7 \mathrm{~Hz} / \mathrm{A}$ for 
$\ell_{e}=13.5 \mathrm{~mm}$ and $2.6 \mathrm{~Hz} / \mathrm{A}$ for $\ell_{e}=10 \mathrm{~mm}$ ). These data indicate that for a given electrode extension, droplet-transfer frequency is linear with current and WFS. Therefore, control of droplet-transfer frequency could be achieved by actuating current and WFS, with the WFS determined by eq (3). The process transfer function can be expressed as

$$
K_{W}=K_{W}\left(\ell_{e}\right)
$$

The transfer functions expressed in eqs (59) and (60) are considered to be static, supported by the following argument. It is assumed that the change in droplet frequency with a change in welding condition occurs within one droplet-detachment period (on the order of 2 to $3 \mathrm{~ms}$ ). Thus, the time required for a change in transfer frequency is much smaller than the time required to determine the change through the PSD calculation ( $3 \mathrm{~ms} \cdot \ll 0.166 \mathrm{~s})$.

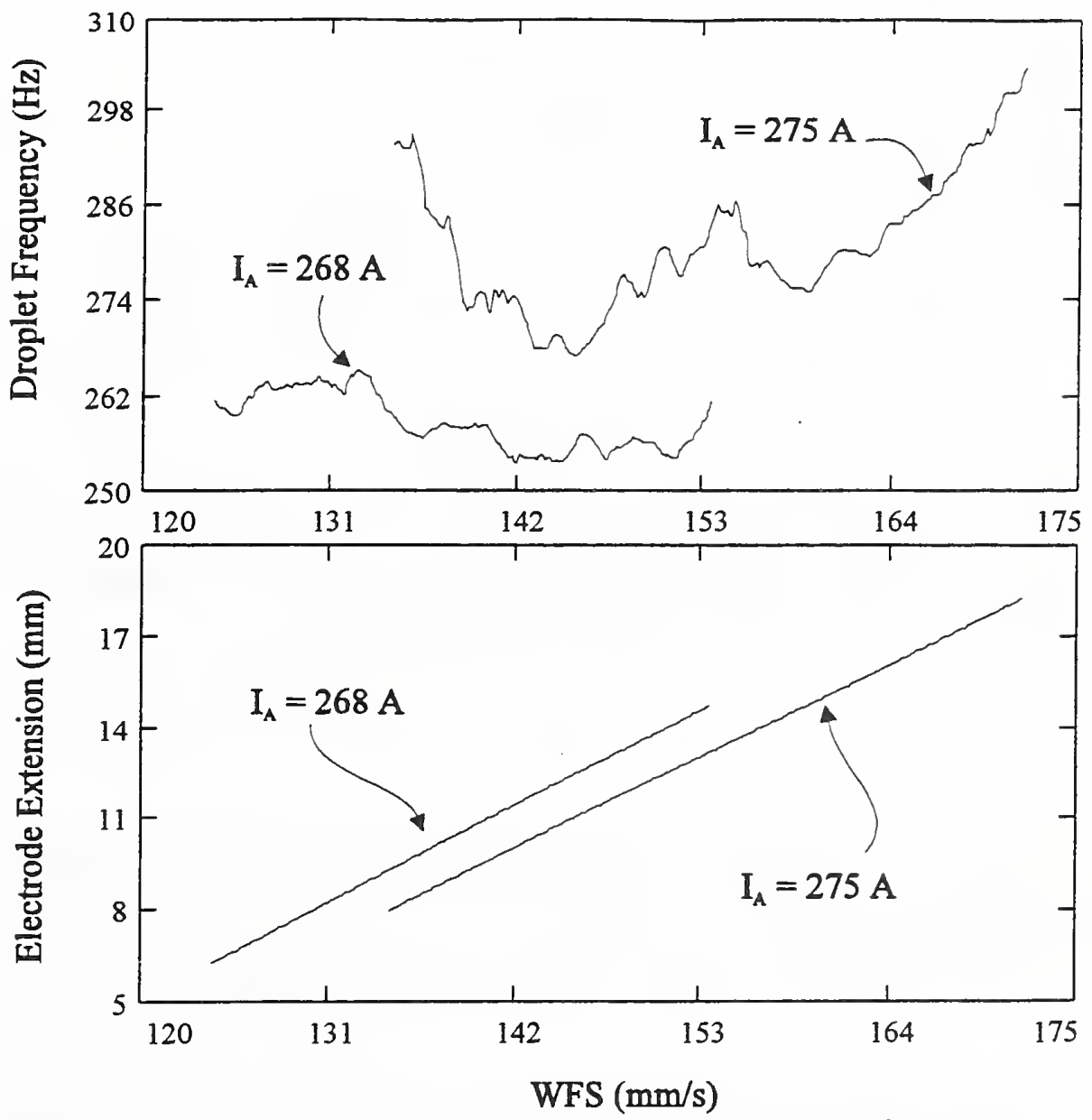

Figure 72. Droplet-transfer frequency and electrode extension as a function of WFS at two values of current. 


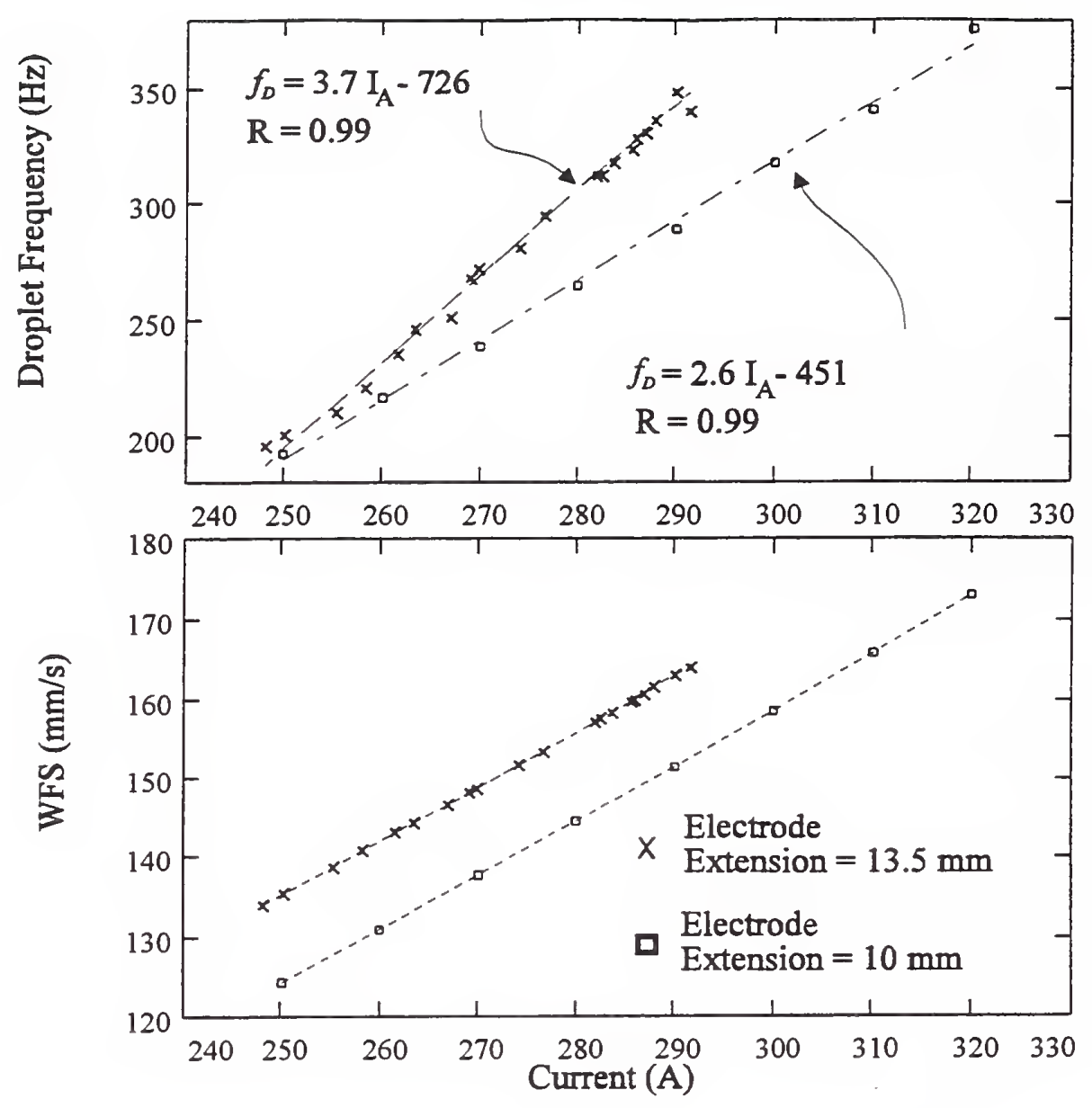

Figure 73. Droplet-transfer frequency and WFS as a function of current for two values of constant electrode extension. The WFS was changed with the current to obtain constant electrode extension.

\subsection{Controller Design}

The PID-type controller was implemented in software on the computer. A block diagram for basic droplet-transfer-frequency control is shown in figure 74 . The controller calculates the welding current from

$$
I_{w}(k)=K_{P} \Delta f(k)+K_{I} \sum_{k} \Delta f(k)+K_{D}[\Delta f(k)-\Delta f(k-1)]+I_{F F} .
$$

where $K_{P}, K_{I}$, and $K_{D}$ are the proportional, integral, and derivative gains; $\Delta f$ is the droplet-transfer-frequency error; $k$ is the discrete time sample; and $I_{F F}$ is the nominal current. The value of $\Delta f$ is calculated from $\left(f_{D}-f_{d}\right)$ where $f_{D}$ is the desired frequency and $f_{d}$ is the measured frequency.

When the controller was operated with constant WFS, $I_{F F}$ was determined from eq (3) for the desired value of electrode extension and WFS. Similarly, when the WFS was actuated simultaneously with the current, the controller calculated the WFS 


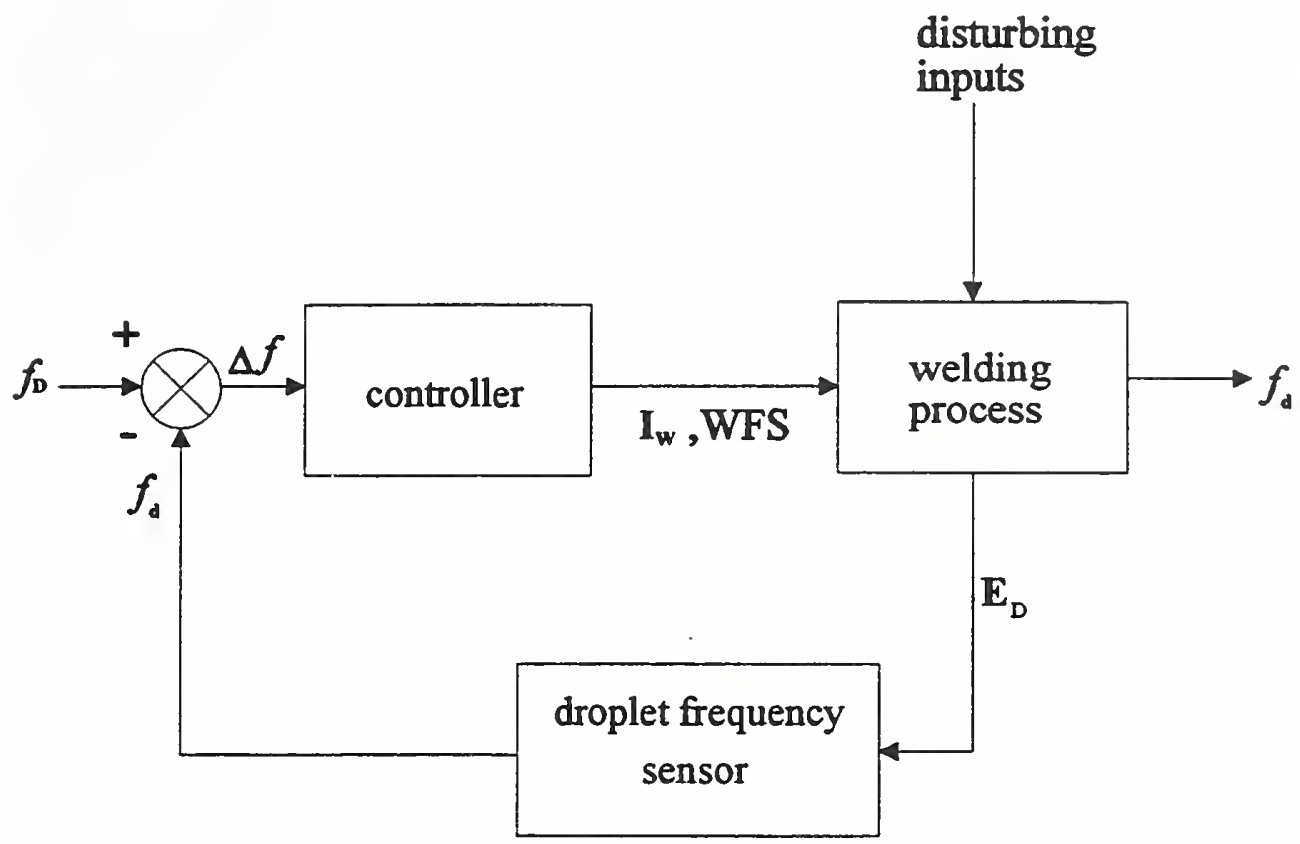

Figure 74. Block diagram of droplet-transfer-frequency control.

from $I_{W}$ and the desired electrode extension. The PSD, transfer-frequency-peak detection, and controller calculations required $0.169 \mathrm{~s}$, providing a control frequency of $5.9 \mathrm{~Hz}$.

The controller gains $K_{P}, K_{I}$, and $K_{D}$ were selected empirically. The current control resolution was $0.25 \mathrm{~A}$. Thus, to respond to a detectable change in frequency, $K_{P}$ was set to $0.25 \mathrm{~A} / 1.46 \mathrm{~Hz}$ or $0.17 \mathrm{~A} / \mathrm{Hz}$. Initial experiments showed that $K_{I}$ $=0.062 \mathrm{~A} / \mathrm{Hz}$ and $K_{D}=0.03 \mathrm{~A} / \mathrm{Hz}$ provided a stable controller response.

\subsection{Results and Discussion}

The results of the control system to a step increase in frequency (260 to $285 \mathrm{~Hz}$ ) actuating only the current are shown in figure 75. The current increased from 282 to 287 A with an overshoot to 291 A. The frequency reached the new setpoint value in $1.75 \mathrm{~s}$ with a steady-state error of nearly 0 . The electrode extension decreased from 10 to $9.2 \mathrm{~mm}$.

The results of a step change in frequency while actuating both current and WFS are shown in figure 76. The frequency achieved the setpoint value in $1.9 \mathrm{~s}$ with little steady-state error. The current climbed to $287 \mathrm{~A}$ from an initial value of $279 \mathrm{~A}$. As expected, the WFS changed in response to the current, from 142 to $147 \mathrm{~mm} / \mathrm{s}$. The electrode extension remained nearly constant at $10 \mathrm{~mm}$. 

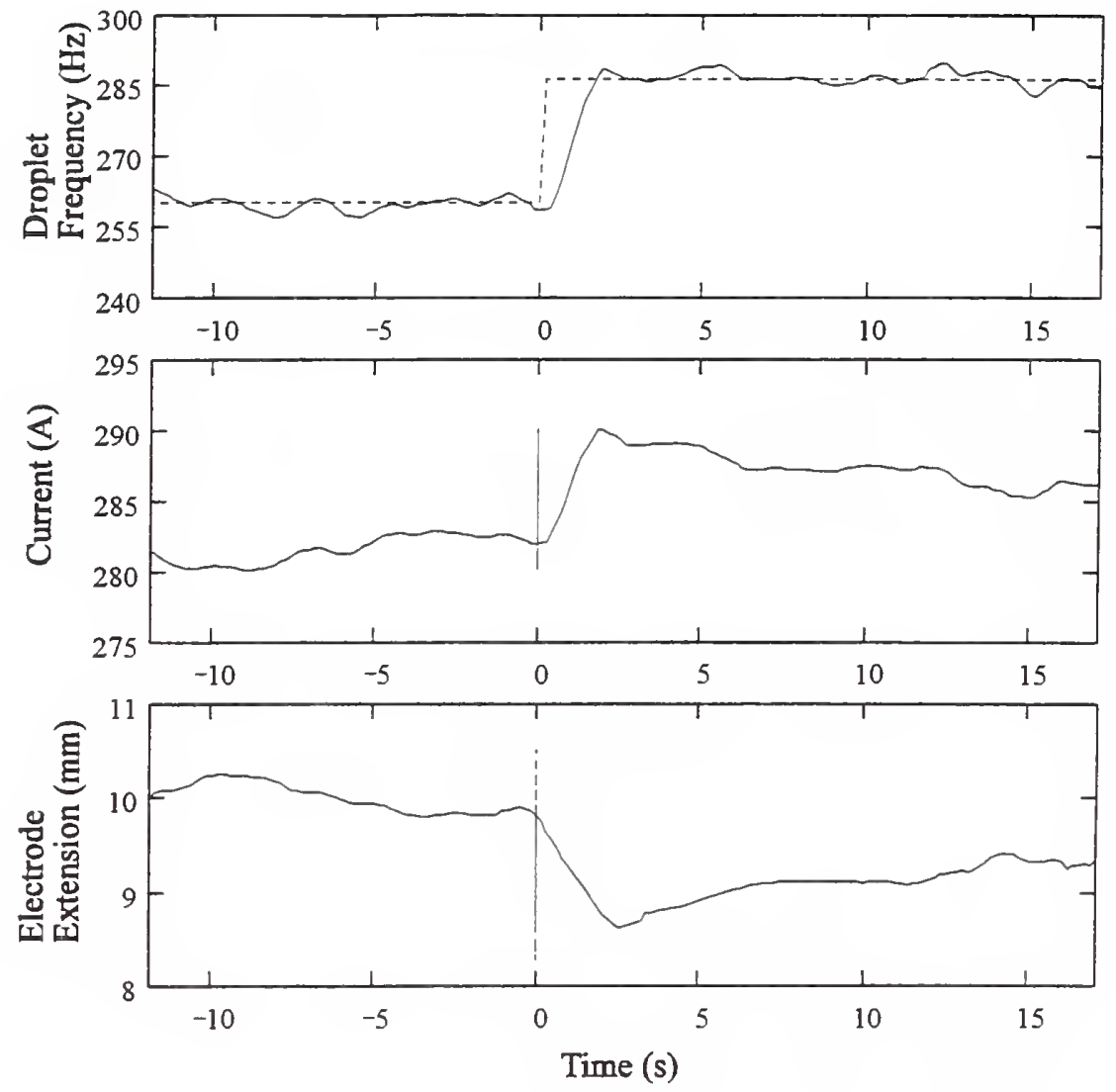

Figure 75. Response of the transfer-frequency controller to a step increase in frequency setpoint with only the current actuated. The WFS was constant at $144 \mathrm{~mm} / \mathrm{s}$.

\subsection{Summary}

A system of droplet-transfer-frequency control using arc-light sensing to measure the actual transfer frequency has been demonstrated. To the knowledge of the authors, this is the first successful demonstration of droplet-transfer-frequency control.

The droplet-transfer frequency increased linearly with current. The frequency also increased linearly with current and WFS when the WFS was slaved to the current. The change in frequency was sensitive to current, on the order of $3 \mathrm{~Hz} / \mathrm{A}$. The rate of frequency increase was a function of WFS and electrode extension. At constant current, the droplet-transfer frequency varied in a nonlinear, nonmonotonic manner with WFS. Thus, droplet-transfer-frequency control based on WFS alone could not be accomplished by a simple linear controller. 
A PID-type controller, actuating current, and the combination of current and WFS can control transfer frequency in response to step changes in the frequency setpoint. The performance of the controller could be improved in two ways: (1) The droplet-transfer frequency could be obtained at a much higher rate with dedicated signal-processing hardware. The resolution and response of the control system could then be increased. (2) A power source with a high-resolution current control is needed, owing to the sensitivity of frequency change with current, to actuate small changes in current when required.

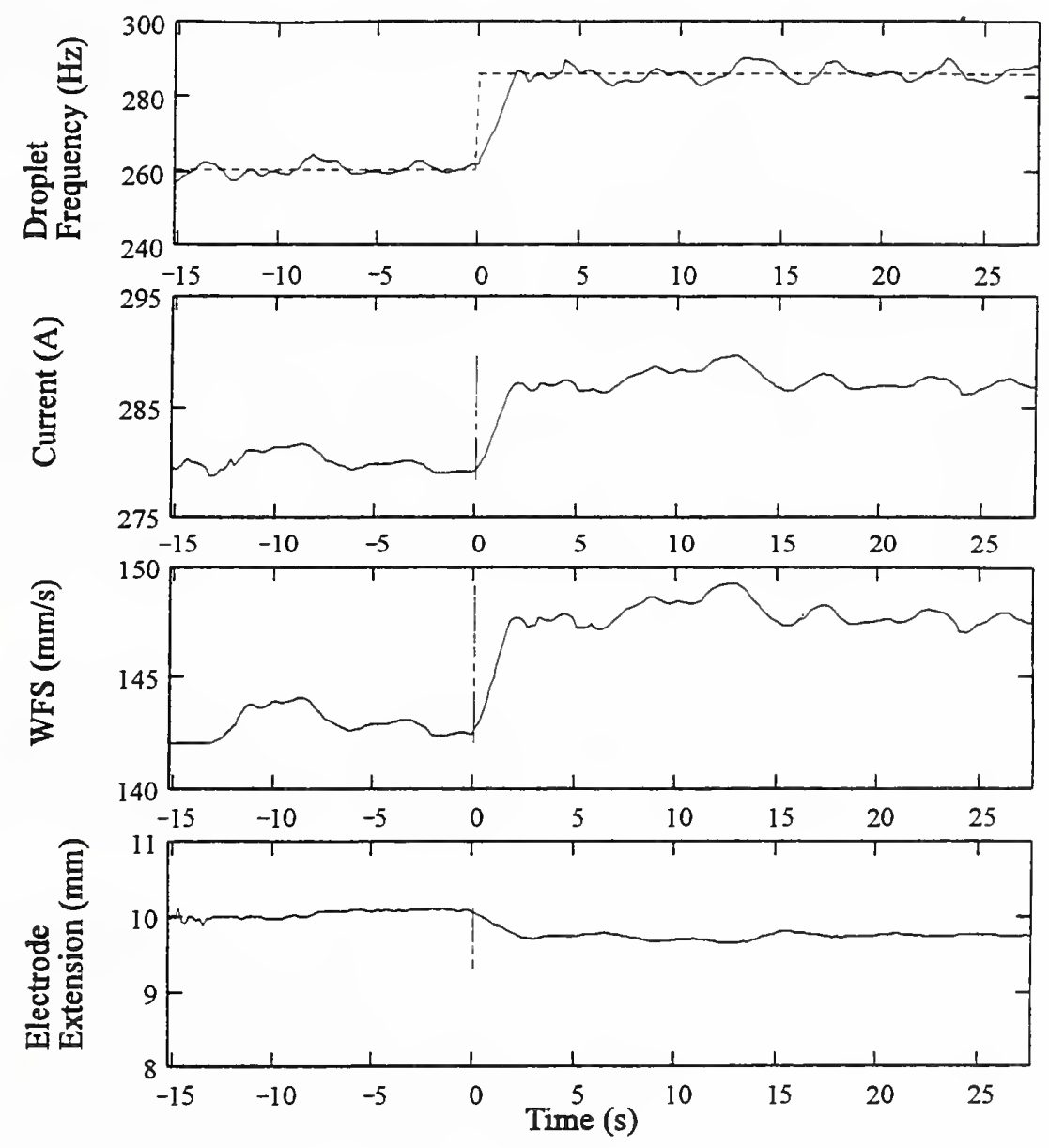

Figure 76. Response of the droplet-transfer-frequency controller to a step increase in frequency setpoint with both current and WFS actuated. 


\section{Conclusions}

To date, it is not possible to achieve true closed-loop control of final weld properties in real time. The principal reason that closed-loop control of weld properties cannot be realized is the lack of appropriate sensors. The approach taken, then, is indirect control of weld properties through better control of the process inputs. Such was the approach taken here. Two parameters of GMAW are known to influence weld quality and were controlled in real time through the use of arc-light sensing: arc length and droplet-transfer frequency.

Arc-light intensity increased with both arc length and arc current. A measure of arc-light intensity was obtained by using a photodiode and by treating the arc as an optically thin, volume source. Arc length was estimated from the detector-output voltage and arc current. Furthermore, the arc-light-detector output varied with droplet detachment; thus, it can be used to detect droplet detachment.

Even though arc length is proportional to arc voltage, the composite nature of GMAW voltage inhibits the estimation of arc length from the process voltage. In response to this limitation, an arc-length sensor that does not require direct measurement of the process voltage was developed. The arc-length sensor was formulated by using established models of arc voltage and observations of power lost by the arc through radiation as a function of arc length and current. The performance of the sensor to estimate arc length was a function of the spectral response of the detector (which could be modified by optical band-pass filters), the orientation of the detector relative to the arc, and shielding-gas composition. The sensor could estimate the arc length within $0.7 \mathrm{~mm}$ while welding with $98 \% \mathrm{Ar}+2 \% \mathrm{O}_{2}$ shielding gas and using a broad, blue-band-pass detector filter.

The advantage of the arc-length sensor developed here is that it operates independently of process voltage and wire-feed speed. On the other hand, it requires the use of a detector external to the process, which poses weld-joint-access problems. Furthermore, the sensor must be calibrated for the given welding conditions and must remain in calibration in the harsh welding environment.

An arc-length controller based on the arc-length sensor and the static and dynamic characteristics of welding was developed. The functions of both the controller and the arc-length sensor were incorporated into the computer software. Arc current was used as a means of process actuation. Results showed that the controller was capable of providing a zero steady-state error in response to step changes in the arclength setpoint and in WFS. The transient response of the fixed-gain controller could be optimized to operate about a given welding condition.

The droplet-transfer frequency during spray-transfer conditions can be determined from variations in the arc-light signal. The variations were directly correlated to droplet-detachment events. Several mechanisms were hypothesized regarding the droplet-detachment signature in the detector signal; a change in arc temperature and, hence, arc radiant intensity, a blockage of a portion of the radiation on the side of the droplet opposite the detector, or a change in arc length and, hence, arc-radiation volume. Existing data supported the possibility that the droplet-detachment signature could be attributed to changes in arc length. It was confirmed that without supplemental information, such as the expected droplet-fransfer-frequency range, it is not 
possible to identify the droplet-transfer frequency from the process-voltage and current waveforms or power spectra. However, the actual droplet-transfer frequency can be obtained in real time simply by locating the frequency of maximum amplitude within the voltage spectrum of the arc-light detector. A 430-nm detector filter, which passes light associated with Fe-emission lines, provided the best droplet detection throughout the spray-transfer regime while welding with $98 \% \mathrm{Ar}+2 \% \mathrm{O}_{2}$ shielding gas.

That the ability to monitor or even control droplet-transfer frequency may improve weld quality is without question. The technique developed here offers the capability of monitoring droplet-transfer frequency in real time. It could be used to map the transfer mode and frequency over a broad range of welding conditions. An advantage of the technique described here is that it relies only on a relative measure of arclight intensity. Furthermore, it is independent of process voltage, current, WFS, and type of power source.

A droplet-transfer-frequency controller was demonstrated based on the transferfrequency-detection scheme. To design the frequency controller, the influence of welding parameters on droplet-transfer frequency was investigated. Transfer frequency was found to be a function of current, WFS, and electrode extension. Transfer frequency increased linearly with current and linearly with current and WFS when the WFS was linked to the arc current. A controller was used to control transfer frequency by actuating either current or current and WFS. The controller was capable of producing a zero steady-state frequency error in response to a step change in the desired transfer frequency. To the knowledge of the authors, this was the first successful demonstration of closed-loop droplet-transfer-frequency control.

R. Bruce Madigan wishes to thank Profs. Stephen Liu, David Olson, Don Williamson, and Jerry Jones (Colorado School of Mines), Prof. Richard W. Richardson (Ohio State University), and Matt Young (NIST).

\section{References}

[1] Committee on Welding Controls. Control of welding processes. NMAB-421, National Materials Advisory Board, National Research Council, U.S. Department of Defense and National Aeronautics and Space Administration; 1987. 58 pp.

[2] O'Brien, R.L. Welding handbook, Vol. 2. Miami, FL: American Welding Society; 1991. 110-155.

[3] Lesnewich, A. Electrode activation for inert-gas-shielded metal-arc welding. Weld. J. 39: 1167-1178; 1955.

[4] Lesnewich, A. Control of melting rate and metal transfer in gas-shielded metal-arc welding: Part 1 - control of electrode melting rate. Weld. J. 8: 343s-353s; 1958.

[5] Lin, M.; Eager, T.W. Influence of arc pressure on weld pool geometry. Weld. J. 64: 163s-169s; 1985.

[6] Tsai, N.S.; Eager, T.W. Distribution of the heat and current fluxes in gas tungsten arcs. Met. Trans. B16: 841-846; 1985.

[7] Lu, M.; Kou, S. Power and current distributions in gas tungsten arcs. Weld. J. 67: 
[7] Lu, M.; Kou, S. Power and current distributions in gas tungsten arcs. Weld. J. 67: $29 \mathrm{~s}-34 \mathrm{~s} ; 1988$.

[8] Johnson, J.A.; Carlson, N.M.; Smartt, H.B.; Clark, D.E. Process control of GMAW: Sensing of metal transfer mode. Weld. J. 70: 91s-99s; 1991.

[9] Lancaster, J.F. The physics of welding, 2nd Ed. New York: Pergamon Press; 1986. 120-142.

[10] Connor, L.P. Welding handbook, 8th Ed., Vol. 1. Miami, FL: American Welding Society; 1987. 31-64.

[11] Halmoy, E.; Brotan, H. Dynamic response of the wire melting rate. International Institute of Welding Public Session, Bratislava, Yugoslavia; 1979.

[12] Waszink, J.H.; van den Heuvel, G.J.P.M. Measurements and calculations of the resistance of the wire extension in arc welding, in Arc physics and weld pool behavior. The Welding Institute International Conference Proceedings. Abington, Cambridge, UK: The Welding Institute; 1979. 227-239.

[13] Quinn, T.P.; Madigan, R.B. Dynamic model of electrode extension for gas metal arc welding, in International trends in welding science and technology. ASM International Conference Proceedings. Metals Park, OH: ASM International; 1992. 1003-1008.

[14] Morrow, R.; Lowke, J.J. A one dimensional theory of the electrode sheaths of electric arcs. J. Phys. D: Appl. Phys. 26: 634-642; 1993.

[15] Lancaster, J.F. The physics of welding, 2nd Ed. New York: Pergamon Press; 1986. 22-24.

[16] Key, J.F.; Chan, J.W.; McIlwain, M.E. Process influence on arc temperature distribution. Weld. J. 62(7): 170s-184s; 1983.

[17] Richardson, R.W. Review of the state-of-the-art of adaptive control for the gas tungsten and plasma arc welding processes. Dayton, $\mathrm{OH}$ : Air Force Materials Laboratory, Wright-Patterson Air Force Base; 1981.

[18] Doeblin, E.O. Dynamic analysis and feedback control. Columbus, OH: Ohio State University; 1962.

[19] D'Azzo, J.J.; Houpis, C.H. Linear control system analysis and design. New York: McGraw-Hill; 1981.

[20] Jones, J.E.; Liu, K.; White, D.R.; Carmein, J.A. Integration of process and control models for intelligent control of welding in International trends in welding science and technology. ASM International Conference Proceedings. Metals Park, OH: ASM International; 1992. 883-887.

[21] Jones, J.E. Weld parameter modelling in International trends in welding science and technology. ASM International Conference Proceedings. Metals Park, OH: ASM International; 1992. 895-898.

[22] Cobine, J.D. Gaseous conductors: theory and engineering applications. New York: McGraw-Hill; 1941.

[23] Llewellyn-Jones, F. Ionization and breakdown in gases. London: Associated Book Publishers Ltd.; 1966.

[24] Penning, F.M. Electrical discharges in gases. New York: Macmillan; 1957. 4-6.

[25] Glickstein, S.S. Arc modelling for welding analysis in Arc physics and weld pool behavior. The Welding Institute International Conference Proceedings. Abington, Cambridge, UK: The Welding Institute; 1979. 1-16.

[26] McKelliget, J.; Szekely, J. Heat transfer and fluid flow in the welding arc. Met. Trans. A17: 1139-1148; 1986. 
[27] Hsu, K.C.; Etemadi, K.; Pfender, E. Study of the free-burning high-intensity argon arc. J. Appl. Phys. 54: 1293-1301; 1983.

[28] Kovitya, P.; Cram, L.E. A two-dimensional model of gas tungsten welding arcs. Weld. J. 65: 34-39; 1986.

[29] Lancaster, J.F. The physics of welding, 2nd Ed. New York: Pergamon Press; 1986. 182-194.

[30] Portillo, D.E.; Moon, T.J. Modelling of radiation in gas-shielded arc welding by the zonal method in Symposium on manufacturing science and engineering, Vol. 64. New York: ASME; 1993. 879-884.

[31] Evans, D.L.; Tankin, R.S. Measurement of emission and absorption radiation by an argon plasma. Phys. Fluids 10: 1137-1144; 1967.

[32] Siegel, R.; Howell, J.R. Thermal radiation heat transfer, 3rd Ed. Washington, DC: Hemisphere Publishing Corporation; 1991.

[33] Peterson, D.W. The calibration and cataloging of spectral emissions from gas metal arc welding of steel from $4100 \AA \dot{A}$ to $7450 \AA$. M.S. Thesis. University of Illinois; 1981 .

[34] Garbuny, M. Optical physics. New York: Academic Press; 1965.

[35] Muller, A.; Greene, W.J.; Rothschild, G.R. Characteristics of inert-gas-shielded metalarcs. Weld. J. 8: 717-727; 1951.

[36] Spraragen, W.; Lengyel, B.A. Physics of the arc and the transfer of metal in arc welding. Weld. J. 1: 2s-42s; 1943.

[37] Kim, E. Visible light emissions during gas tungsten arc welding. M.S. Thesis. Cambridge, MA: Massachusetts Institute of Technology; 1986.

[38] Ghent, H.W.; Roberts, D.W.; Hermance, C.E.; Kerr, H.W.; Strong, A.B. Arc efficiencies in TIG.welds in Arc physics and weld pool behavior. The Welding Institute International Conference Proceedings. Abington, Cambridge, UK: The Welding Institute; 1979. 17-23.

[39] Alum, C.J. Power dissipation in the column of a TIG welding arc. Phys. D: Appl. Phys. 16: 2149-2165; 1983.

[40] Deam, R.T.; Drew, P.N. Relationship between arc light, current and arc length in TIG welding in Advances in joining and cutting processes. The Welding Institute Conference Proceedings. Abington, Cambridge, UK: The Welding Institute; 1989. 33-40.

[41] Dereniak, E.L.; Crowe, G. Optical radiation detectors. New York: Wiley; 1984.

[42] Kim, E.W.; Allemand, C.; Eager, T.W. Visible light emissions during gas tungsten arc welding and its application to weld image improvement. Weld. J. 66: 369s-377s; 1987.

[43] O'Brien, R.L. Physics of welding, chapter 4 in Welding handbook, 8th Ed., Vol. 1. Miami, FL: American Welding Society; 1991. 127.

[44] Fujimura, H.; Ide, E.; Inoue, H. Estimation of contact tip-work piece distance in gas metal arc welding. Weld. Intern. 6: 522-528; 1988.

[45] Conrardy, C. Control of GMAW with coaxial vision. M.S. Thesis. Ohio State University; 1991.

[46] Hunter, J.J.; Bryce, G.W.; Doherty, J. On-line control of the arc welding process in Developments in mechanized automated and robotic welding. The Welding Institute Conference Proceedings: Paper 37. Abington, Cambridge, UK: The Welding Institute; 1980.

[47] Fellure, J.E.; Leslie, E.L. Arc length measurement and control by optical scanning. U.S. Patent 4093844. 1978.

[48] Johnson, C.A.; Sciaky, A.M. System for controlling length of welding arc. U.S. Patent 3236997. 1966. 
[49] Sciaky, A.M.; Vilkas, E.P. Welding control system. U.S. Patent 3262006. 1966.

[50] Romanenkov, E.I. Control of arc length on the basis of its spectral radiation. Svar. Proiz. 9: 50-51. Translated in Weld. Prod. 23: 53-54; 1976.

[51] Deam, R.T.; Drew, P.N. Relationship between arc light, current and arc length in TIG welding in Advances in welding and cutting processes. The Welding Institute Conference Proceedings. Abington, Cambridge, UK: The Welding Institute; 1989. 33-40.

[52] Madigan, R.B.; Quinn, T.P. Sensing of gas metal arc welding process characteristics for welding process control. U.S. Patent No. 5,349,156. 20 September 1994.

[53] Madigan, R.B.; Quinn, T.P.; Siewert, T.A. Sensing droplet detachment and electrode extension for gas metal arc welding in International trends in welding science and technology. ASM International Conference Proceedings. Metals Park, OH: ASM International; 1992. 999-1002.

[54] Wu, G.D.; Richardson, R.W. The dynamic response of self-regulation in the welding arc in Recent trends in welding science and technology. ASM International Conference Proceedings. Metals Park, OH: ASM International; 1989. 929-931.

[55] Dillenbeck, V.R.; Castagno, L. The effects of various shielding gases and associated mixtures in GMA welding of mild steel. Weld. J. 66: 45-49; 1987.

[56] O'Brien, R.L. Welding handbook, 8th Ed., Vol. 2. Miami, FL: American Welding Society; 1991. 87.

[57] Halmøy, E. The pressure of the arc acting on the weld pool in Arc physics and weld pool behavior. The Welding Institute International Conference Proceedings. Abington, Cambridge, UK: The Welding Institute; 1979. 259-266.

[58] O'Brien, R.L. Welding handbook. 8th Ed., Vol. 2. Miami, FL: American Welding Society; 1991. 109-130.

[59] Ogilvie, K.S. Modelling of the gas metal arc welding process for control of arc length. Ph.D. Dissertation. University of Michigan, Ann Arbor, MI. 1991.

[60] Quinn, T.P.; Madigan, R.B. Adaptive arc length controller design for GMAW in Modelling and control of joining processes. American Welding Society International Conference Proceedings. Abington, Cambridge, UK: The Welding Institute; 1993.

[61] Lancaster, J.F. The physics of welding, 2nd Ed. New York: Pergamon Press: 1986. 228-305.

[62] Liu, S.; Siewert, T.A.; Adam, G.A. Arc welding process control from current and voltage signals in Computer technology in welding. The Welding Institute Conference Proceedings. Abington, Cambridge, UK: The Welding Institute; 1990. 26-35.

[63] Johnson, J.A.; Carlson, N.M.; Smartt, H.B. Detection of metal transfer mode in GMAW in Recent trends in welding science and technology. ASM International Conference Proceedings. Metals Park, OH: ASM International; 1989. 377-381.

[64] Arata, Y.; Inoue, K.; Futamata, M.; Toh, T. Investigation on welding arc sound effect of welding method and welding condition of welding arc sound. Trans. Jpn. Weld. Res. Inst. 8: 1; 1979.

[65] Matteson, M.A.; Morris, R.A. An optimal artificial neural network for GMAW arc acoustic classification in International trends in welding science and technology. ASM International Conference Proceedings. Metals Park, OH: ASM International; 1992. 1031-1035.

[66] Heald, P.R.; Madigan, R.B.; Siewert, T.A.; Liu, S. Mapping the droplet transfer modes for an ER100S-1 GMAW electrode. Weld. J. 73: 38s-44s; 1993.

[67] Bendant, J.S.; Piersol, A.G. Random data: analysis and measurement procedures. New York: Wiley-Interscience; 1971. 22-25. 
[68] Dunn, G.J.; Eager, T.W. Metal vapors in gas tungsten arcs: Parts I and II. Met. Trans 17A: 1851-1871; 1986.

[69] O'Brien, R.L. Welding handbook, 8th Ed., Vol. 2. Miami, FL: American Welding Society; 1991. 1-44.

[70] Lesnewich, A. Control of melting rate and metal transfer in gas shielded metal arc welding. Weld. J. 37: 343s-418s; 1958.

[71] Kim, Y.-S.; Eager, T.W. Analysis of metal transfer in gas metal arc welding. Weld. J. 72: 269s-278s; 1993. 


